\title{
A cosmopolitan outlook on health workforce development
}

Citation for published version (APA):

van de Pas, R. A. M. (2020). A cosmopolitan outlook on health workforce development. [Doctoral Thesis, Maastricht University]. Global Academic Press. https://doi.org/10.26481/dis.20200914rp

Document status and date:

Published: 01/01/2020

DOI:

10.26481/dis.20200914rp

Document Version:

Publisher's PDF, also known as Version of record

\section{Please check the document version of this publication:}

- A submitted manuscript is the version of the article upon submission and before peer-review. There can be important differences between the submitted version and the official published version of record.

People interested in the research are advised to contact the author for the final version of the publication, or visit the DOI to the publisher's website.

- The final author version and the galley proof are versions of the publication after peer review.

- The final published version features the final layout of the paper including the volume, issue and page numbers.

Link to publication

\footnotetext{
General rights rights.

- You may freely distribute the URL identifying the publication in the public portal. please follow below link for the End User Agreement:

www.umlib.nl/taverne-license

Take down policy

If you believe that this document breaches copyright please contact us at:

repository@maastrichtuniversity.nl

providing details and we will investigate your claim.
}

Copyright and moral rights for the publications made accessible in the public portal are retained by the authors and/or other copyright owners and it is a condition of accessing publications that users recognise and abide by the legal requirements associated with these

- Users may download and print one copy of any publication from the public portal for the purpose of private study or research.

- You may not further distribute the material or use it for any profit-making activity or commercial gain

If the publication is distributed under the terms of Article $25 \mathrm{fa}$ of the Dutch Copyright Act, indicated by the "Taverne" license above, 


\section{A cosmopolitan outlook}

\section{on health workforce development}

Remco van de Pas

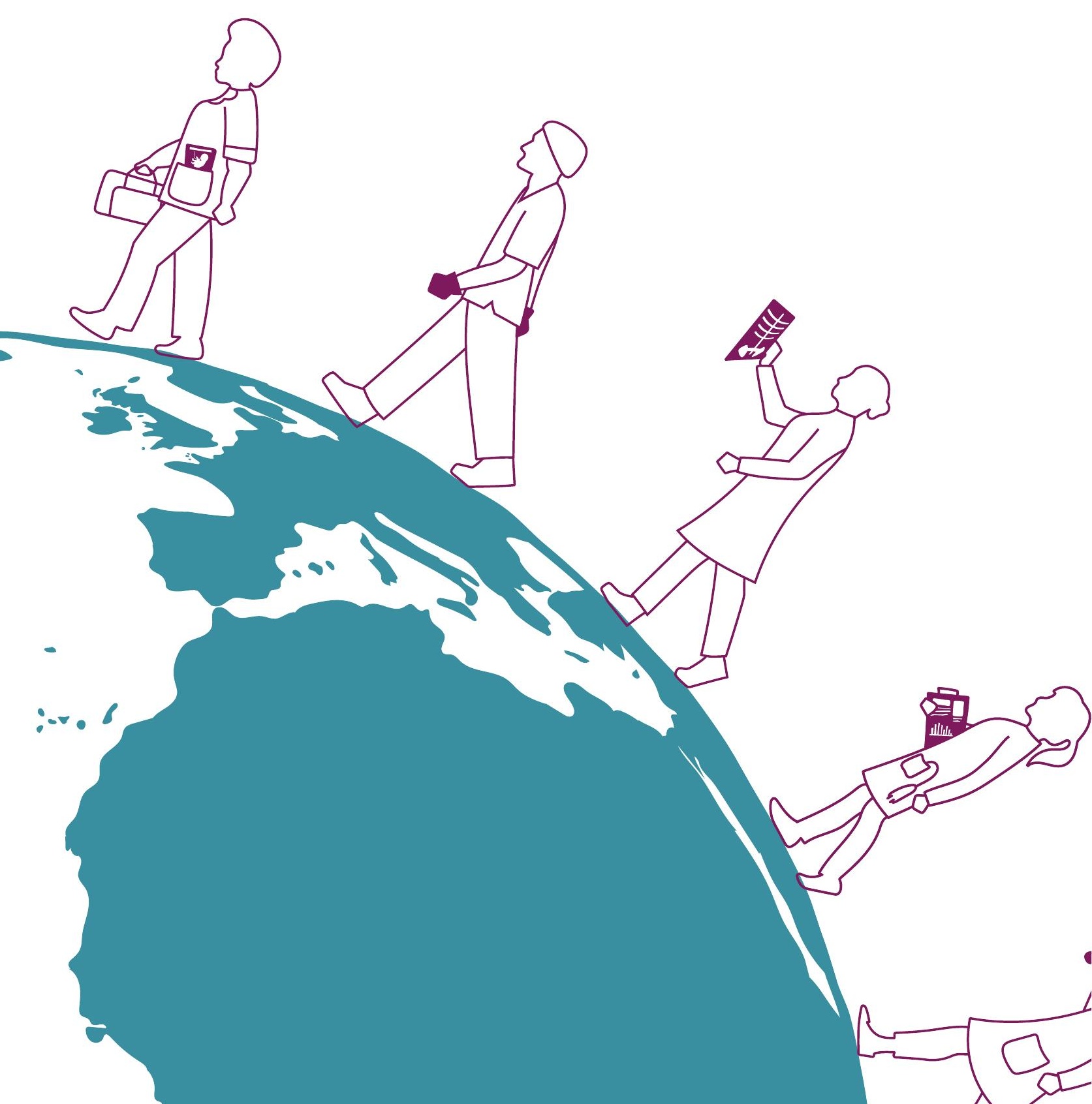



A cosmopolitan outlook on health workforce development

Remco van de Pas 


\section{COLOFON}

\section{A cosmopolitan outlook on health workforce development}

\section{Dissertation}

Remco van de Pas

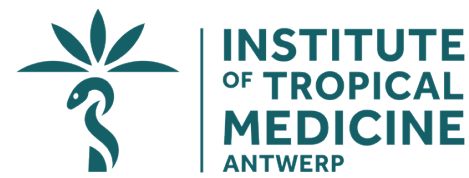

\section{Maastricht University}

Cover and layout: Lize Jansen (lizejansen.nl)

Printing: ProefschriftMaken

Edit: Ritwik Dahake

Author info: r.vandepas@posteo.de

Van de Pas R. A cosmopolitan outlook on health workforce development. 2020. 353 p. Global Academic Press. ISBN: 978-94-6380-922-1

All rights reserved. No part of this publication may be reproduced, stored in a retrieval database or published in any form or by any means, electronic, mechanical or photocopying, recording or otherwise, without the prior written permission of the author or, when appropriate, the publishers of the papers.

The research presented in this thesis was conducted at CAPHRI Care and Public Health Research Institute, Department of Health, Ethics and Society of Maastricht University. CAPHRI participates in the Netherlands School of Public Health and Care Research CaRe.

The research presented in this thesis was also conducted at, and financed by, the Department of Public Health, Institute of Tropical Medicine, Antwerp, Belgium. 


\title{
A cosmopolitan outlook on health workforce development
}

\author{
DISSERTATION
}

to obtain the degree of Doctor at the Maastricht University,

on the authority of the Rector Magnificus Prof.dr. Rianne M. Letschert

in accordance with the decision of the Board of Deans,

to be defended in public on

Monday, the 14th of September 2020 at $16.00 \mathrm{hrs}$.

By Remco Adrianus Maria van de Pas 


\section{SUPERVISORS:}

Prof. dr. D.M.R. Townend, Maastricht University

Prof. dr. J.S.M. Krumeich, Maastricht University

\section{CO-SUPERVISOR:}

Prof. dr. W. Van Damme, Institute of Tropical Medicine, Antwerp, Belgium

ASSESSMENT COMMITTEE:

Prof. dr. K. Horstman (chair)

Dr. T. Krafft

Prof. dr. R. Labonté, University of Ottawa, Canada

Prof. dr. D. Lesage, Ghent University, Belgium

Dr. P. Schröder-Bäck 
to my daughter Ika van de Pas 
Chapter 1: Introduction and Problem Outline $\quad 15$

\begin{tabular}{lll}
1.1 & The global health workforce challenge & 16 \\
\hline
\end{tabular}

\begin{tabular}{lll}
\hline 1.2 & Cosmopolitanism & 18
\end{tabular}

\begin{tabular}{ll}
1.2 .1 A cosmopolitan outlook & 20 \\
\hline
\end{tabular}

$\begin{array}{ll}1.2 .2 & \text { A cosmopolitan outlook on health workforce migration }\end{array}$

$\begin{array}{lll}1.3 & \text { Global health policy frames } & 23\end{array}$

1.4 Health equity in a globalizing era of deep economic integration $\quad 24$

$\begin{array}{lll}1.4 .1 & \text { The health labor market } & 25\end{array}$

1.5 Global health security and the securitization of health 26

\begin{tabular}{lll}
1.6 & Global health governance & 28 \\
\hline
\end{tabular}

\begin{tabular}{llr} 
1.6.1 Globalization paradox & 30 \\
\hline
\end{tabular}

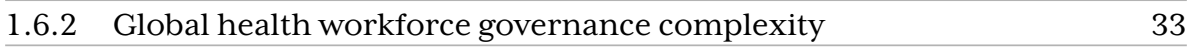

1.6.3 Gridlock in global cooperation for health 35

$\begin{array}{lll}1.7 & \text { Research questions } & 37\end{array}$

1.8 Conceptualizing global health workforce governance and its paradox 38

1.9 Outline 43

$\begin{array}{ll}\text { Chapter 2: Methodology } & 47\end{array}$

$2.1 \quad$ Methodological approaches studying global health $\quad 48$

2.2 Differentiating methodologies in studying globalization and health $\quad 49$

2.3 Methodological cosmopolitanism 50

2.4 Health workforce development and its governance 51

2.5 Pluriform methodologies $\quad 53$

2.6 Methodological limitations and challenges 56

Chapter 3: Governance of the global health workforce 59

3.1 Health workforce development and retention in Guinea:

$\begin{array}{ll}\text { a policy analysis post-Ebola } & 61\end{array}$

3.2 Tracing the policy implementation of commitments made by national governments and other entities at the Third Global Forum on Human $\begin{array}{ll}\text { Resources for Health } & 81\end{array}$

Chapter 4: Global health workforce governance $\quad 101$

4.1 Democratizing the World Health Organization 103

4.2 The Code of Practice and its enduring relevance in Europe $\begin{array}{ll}\text { and Eastern and Southern Africa } & 117\end{array}$

Chapter 5: Global governance of the health workforce 133

5.1 The securitization of health and humanitarian space:

Health workers under attack? 135

5.2 Ebola, the epidemic that should never have happened 159

5.3 Interrogating resilience in health systems development 167 
Chapter 6: Global health policy and the cosmopolitan outlook

6.1 Global health governance in the sustainable development goals:

Is it grounded in the right to health?

6.2 Global Health in the Anthropocene: Moving Beyond Resilience and Capitalism

6.3 Framing the Health Workforce Agenda Beyond Economic Growth

209

Chapter 7: Discussion 221

7.1 Domestic policy space to expand and reform the health workforce 223

7.1.1 International finance 224

7.1.2 Economic reforms and fiscal space limitations 225

7.2 Democratic legitimacy 229

7.2.1 The democratic legitimacy of the World Health Organization 229

7.2.2 The governance of international health workforce migration 231

7.3 International policy trends outside the global health domain 233

7.3.1 The securitization of health and its impact on the health workforce 234

$\begin{array}{lll}7.3 .2 & \text { The legitimacy of the health care workers } & 236\end{array}$

$\begin{array}{lll}7.3 .3 & \text { Structural adjustment policies } & 237\end{array}$

$\begin{array}{ll}\text { 7.3.4 Trade agreements and their impact } & 240\end{array}$

7.4 Limited cosmopolitan space for global health workforce development 241

7.4.1 Global health governance grounded in the Right to Health 242

7.4.2 Synergies and tensions between UHC and global health security $\quad 244$

\begin{tabular}{lll}
\hline 7.4 .3 & Complex adaptive systems & 246 \\
\hline 7.4 .4 &
\end{tabular}

$\begin{array}{lll}\text { 7.4.4 Exerting power in global health governance } & 247\end{array}$

7.5 The political trilemma and global health workforce governance 250

$\begin{array}{lll}7.6 & \text { Reflections on conceptual framework and methodology } & 257\end{array}$

7.6.1 Boundary spanning, multi-perspectivism and epistemic limitations 261

7.7 A cosmopolitan outlook on health workforce development 266

$\begin{array}{ll}\text { References } & 277\end{array}$

$\begin{array}{ll}\text { Annex 1: Valorization Addendum } & 319\end{array}$

Annex 2: Summary 327

Annex 3: Curriculum Vitae 331

Annex 4: Acknowledgements 343

$\begin{array}{ll}\text { Annex 5: Abbreviations } & 349\end{array}$

Tables

Table 3.1: Numbers of interviews conducted per groups of participants $\quad 66$

Table 3.2: Health workforce profiles and distribution in Forecariah and Yomou districts

Table 5.1: Overview of four roles in four dimensions of health work in violent conflicts 


\section{Figures}

Figure 1.1: Global Health Governance along three political spaces 29

Figure 1.2: The Political trilemma of the World Economy 30

Figure 1.3: The Political Trilemma and Global Health Workforce Governance $\quad 39$

Figure 3.1: Evolution of HRH policy in Guinea Post-Ebola 65

Figure 3.2: Geographic distribution of health training institutions in Guinea, April $2017 \quad 73$

Figure 3.3: Distribution of graduates from 2010 to 2015 by staff category 73

Figure 3.4: Distribution of graduates from 2010 to 2015 by type of institution (private or public) 74

Figure 7.1: The Political Trilemma in relation

to Global Health Workforce Governance 251

Figure 7.2: Shortfalls and overshoots in the Doughnut 268

\section{Boxes}

Box 3.1: Reforms by the Guinean government in HW policy and management functions 64

Box 3.2: Results of the implementation of Human Resources for Health actions 86

Box 3.3: Results of the initiated policy and governance mechanisms $\quad 89$

Box 3.4: Examples of actions according to the Availability, Accessibility, Acceptability and Quality dimensions and policy pathways $\quad 90$

Box 3.5: Examples of monitoring mechanisms being initiated 91

Box 3.6: Examples of Human resources for Health governance mechanisms being initiated $\quad 92$

Box 3.7: Examples of the Human resources for Health political momentum 93

Box 3.8: Examples of Human resources for Health actions by other entities $\quad 94$ 


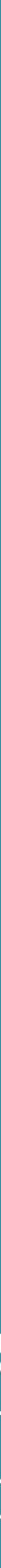




\section{PREFACE}

"I advance two steps, it goes two steps backward. I take ten steps and the horizon moves ten steps forward. No matter how far I walk, I will never reach it. What is the use of utopia? That's its use: to help us walk." (Galeano 1993) 
The last words of this doctoral thesis were written in the early days of 2020. At the time, it was unimaginable that 6 months later, a Coronavirus pandemic would already have affected over 15 million people, and led to more than 600.000 deaths. The still raging Covid-19 pandemic is one of those rare and unique periods in time that profoundly shakes up people's professional and personal lives. Mobility, economies and social lives have been paralyzed across the world. Health care services have been overwhelmed. All around the globe, the pandemic provides a shock momentum of which ripple effects, both negative and positive ones, will be felt for the coming decade.

Informal and formal health care workers are the sung, but still more often unsung, 'heroes' of this pandemic as they provide the frontline health care for patients, families and communities. Their importance as key public servants in societies has become very clear. But, these health workers are also human beings and citizens in their respective countries. They carry a disproportional risk to be infected with SarsCoV-2 themselves. The huge emotional and physical strain many face, deserves to be recognized. Health care workers may experience anguish, pain, anger, trauma, sadness, mourning, helplessness, relief, but also a sense of connectivity and purpose. This period may have scarred them. The whole crisis will hopefully lead to a thorough reflection on the core function and values of care work in our societies. At best, it will transform the profession. Among the overwhelming amount of scientific articles and reports in news media on the coronavirus pandemic, relatively limited attention has been paid to the humane and professional aspects of the health systems that ought to prepare for and respond to such global health risks. In all the heated debates on virus transmission models, vaccine development, lockdowns, face masking, economic recovery and the politics of global health governance, the perspective of health workers and their interaction with patients and society, sometimes gets neglected.

And that's a pity, as the impact of the pandemic, including the role of the health workforce, differs much according to context, country and social position. Workforce development is much related to the availability of decent employment, accessibility to and investment in public services, gender equity, economic stability and a secure living environment. It is a matter of politics and social justice to meet the structural conditions enabling health workers to play a fulfilling and meaningful role in communities and health care settings. A complex interplay of factors influence the outcomes in workforce development; among others, its governance, social policies, powers, actors, public health needs and the political- economy. In that sense, the Covid-19 pandemic functions as a magnifying glass that amplifies the paradoxes of modern globalization and late-stage capitalism. In spite of positive developments in many fields, health and wealth inequalities have increased in and between countries. In many places health systems and social programs have been crippled by austerity 
and neglect. Economic growth has been instable and unbalanced, impoverishing many across the globe. The climate crisis deepens, leading to more violent conflict, refugee movements and has a direct as well as indirect impact on public health outcomes. Health workers may have a role in, and are impacted by, these global trends. Such forces contribute to the increased international migration of health care workers. Improved health systems governance, management, innovation and scientific developments can support in overcoming these complex challenges. However, at the root, it is also the historical injustices, violence, pollution, racism and coloniality of power that have shaped health systems and societies to what they are nowadays. For this reason a cosmopolitan, transnational, analytical lens has been chosen for this study. National and local health systems, including their labor workforce, and international organizations do not function in isolation. They are part of a complex interplay.

At this particular moment in time, in the midst of a pandemic, I believe that we have arrived at a crossroads. Some will long for the old order and security of the nation state, while others will want to move forward with a transition towards fair societies and stable and ecologically balanced economies. Before charting paths on how to move forward, there must also be a better understanding of not only what ought to be done to strengthen health systems, but also to clarify the existing structural obstacles, including the powers, politics and problems that sustain a gridlock of impoverished health services in too many places in this world. The right questions need to be asked before any solution is feasible.

The Coronavirus pandemic sheds light, a cosmopolitan outlook even, on this interconnectedness and the inherent fragility we face as humanity living together. These are not only large issues in small places. There is a growing understanding of how planetary boundaries and economic globalization limit the room for manoeuvre of nations to develop their health and social systems. Principles of solidarity and shared responsibility between countries are required to develop the global health workforce in an equitable manner. Health personnel are central to the care and wellbeing that is so often denied at the individual and societal level. My main aim is that this work contributes to a better understanding of, and policy actions for, health workforce development and international cooperation in this area. History tells us that social developments do not appear gradually. Rather, they follow periods of shocks and conflict whereby a political window appears facilitating new initiatives. The Covid-19 pandemic could turn out such a global momentum.

"Epidemics that are associated with poverty in other parts of the world may one day provoke concerted action by wealthy countries to eradicate the conditions of poverty that caused the spread of disease on a world scale." (De Swaan, 1998) 


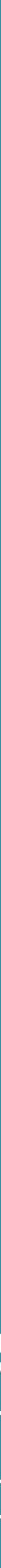


Introduction and Problem Outline

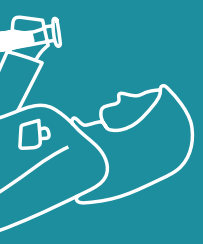

\section{CHAPTER 1: \\ ion and Problem Outline

(n)

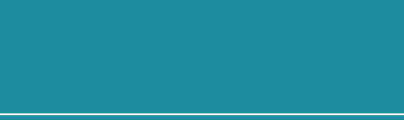

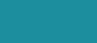

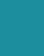

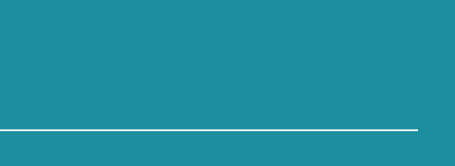

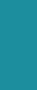

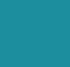

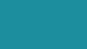

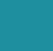

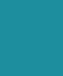

(

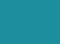

\section{(}

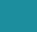

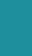
0 


\subsection{THE GLOBAL HEALTH WORKFORGE CHALLENGE}

Over the last few decades, the global health workforce (HWF) gap has increased. By this gap, we mean the skilled HWF required providing essential health care services across the world in an equitable manner. Due to demographic growth in different regions of the world, an aging workforce and an epidemiological transition to chronic diseases worldwide, there is a need for, and impetus required, to invest in skilled health workers and their decent employment. The World Health Organization (WHO) estimates that 4.45 health workers per 1,000 population are required to reach the Sustainable Development Goals (SDGs) health-related targets. This amounts to a total global deficit of 17.6 million health workers relative to current supply, with a projected deficit of 13.6 million health workers in Low- and MiddleIncome Countries (LMICs) alone. (Liu et al. 2017)

The Ebola outbreak in West Africa from 2014-2015 showed how vulnerable health systems are when a skilled workforce with core capacities for epidemic response is missing. It has led to a renewed notion that there is an urgent requirement for the international community and national governments to invest in and develop the global workforce. (Sidibé and Campbell 2015) Efforts to develop sufficient, qualitative and equally distributed Human Resources for Health (HRH) have had limited success, although positive country examples do exist. Inequalities regarding HWF distribution within and between countries remain a problem with a view on building strong health systems. This global challenge is most drastic in, but not restricted to LMICs. It is an interconnected issue for countries as HWF mobility relates to the larger challenge of managing labor migration and health employment. The international migration of health care workers adds to drivers of health systems inequalities such as urbanization, demographic transition and HWF attrition to private health care services or even away from the health sector. (Labonté and Ruckert 2019, pp. 200-01) Given the need to develop Essential Public Health Service as well as the global drive towards Universal Health Coverage (UHC) as part of the SDGs, it is remarkable that HWF development is not a top priority for the global health policy agenda. Limited HWF capacity is a major bottleneck in having health systems functioning properly. It has been estimated that 100 countries, representing one-third of the world's population, do not meet the different, International Labour Organisation (ILO) standards of 34.5 skilled health professionals per 10,000

\footnotetext{
1 On 1 January 2016, the 17 Sustainable Development Goals (SDGs) of the 2030 Agenda for Sustainable Development-adopted by world leaders in September 2015 at a historic UN Summitofficially came into force. Over the next fifteen years, with these new Goals that universally apply to all, countries will mobilize efforts to end all forms of poverty, fight inequalities and tackle climate change, while ensuring that no one is left behind. https://sustainabledevelopment.un.org/
} 
population required for providing essential health services. (Scheil-Adlung 2013) A key question is then why countries do not take more assertive and collaborative action in addressing this crucial global health challenge?

In the $21^{\text {st }}$ century, health systems have rapidly become globalized. (Labonté and Ruckert 2019, pp. 164-91) Adequate and democratic governance at national and sub-national levels is not sufficient anymore to address the workforce challenges. Firstly, more actors and policy domains got involved in international health systems development. Secondly, the HWF challenge itself is increasingly global and complex and requires representative institutions, frameworks and governance processes at the global level to redress inequalities and shortages in the workforce. Thirdly, the norms, values and political choices shaping the development of the workforce are increasingly transnationally determined.

Increased global interdependence and interconnectedness in facing health challenges led scholars in Global Health Governance (GHG) to consider that a cosmopolitan approach towards policymaking is required in order to strengthen health systems in a sustainable manner. Today, 'global health' is considered critical for national and international security, domestic and global economic well-being and economic and social development in less developed countries is also a major growth sector of the global economy... It requires the strategic link with other transnational agendas and a strengthening of the political ability to position health interests and defining and selecting political spaces. (Kickbusch and Reddy 2015)

In this thesis, I provide the argument that a cosmopolitan approach, or better, a cosmopolitan outlook to HWF development is required to overcome current health system challenges within and between countries. This would consecutively lead to advancing shared responsibilities ${ }^{2}$, and investments being committed to, by countries and other relevant actors in developing and securing accessibility to a fit-for-purpose skilled HWF whose services are accessible and affordable for the population. Necessarily then, this study also analyses and deconstructs the reasons as to why HWF development has so far been mainly approached from an 'explanatory nationalism' angle based on security and economic considerations. Political choices, historic pathways, power differentials in and between countries as well as institutional paucity are among the factors that have led to such an

\footnotetext{
2 More precisely, this should follow the principle of 'Common But Differentiated Responsibilities (CBRD)'. For its application in relation to the SDG's implementation, see: Nobbe, C. (2015). Universality, common but differentiated responsibilities and the Sustainable Development Goals. Institute for International and Security Affairs-Working Paper FG 8 2015, 1, 5 https://hr.un.org/sites/hr.un.org/files/Universality\%2C\%20common\%20but\%20differentiated\%20 responsibilities $\% 20 \% 26 \% 20$ Sustainable\%20Development\%20Goals_0.pdf
} 
'avoidable dystopia'. ${ }^{3}$ The study will eventually reflect on whether there could be, and if so how, to advance a cosmopolitan moment in advancing HWF development, rooted in principles such as solidarity and health equity, whilst upholding human rights.

In the next section, I will clarify cosmopolitanism and its roots in moral philosophy as well as how sociologist Ulrich Beck has defined a cosmopolitan outlook on addressing global challenges. I will clarify what such a cosmopolitan outlook would imply for HWF development and more specifically for the governance of health labor migration. Afterward, I will outline why global health (workforce) cooperation is mainly driven by other values than cosmopolitanism and how this is approached via different policy imperatives.

\subsection{COSMOPOLITANISM}

Cosmopolitanism is a political philosophy theory and concept that maintains that there are moral obligations owed to all human beings based solely on our humanity alone. Cosmopolitanism is based on a notion of common humanity that translates ethically into an idea of shared or common moral duties towards others by virtue of this humanity. (Garrett W Brown and Held 2010, p. 1) Based on this, cosmopolitans generally posit three corresponding moral and normative commitments."First, cosmopolitans believe that the primary units of moral concern are individual human beings above states or other forms of communitarian or political association. Second, cosmopolitans maintain that this moral concern for individuals should be equally applied. Third, cosmopolitanism is universal in its scope, maintaining that all humans are equal in their moral standing and that this moral standing applies to everyone everywhere as if we are all citizens of the world." (Brown and Held 2010, pp. 1-2) Cosmopolitanism enjoys a long history and it origins can be found with the Cynics and the Stoic tradition, notably Diogenes of Sinope (400-323 BC) who insisted that he was a 'citizen of the world' (kosmopolites). (Brown and Held 2010, p. 4) The most prominent philosopher during the Enlightenment who propagated contemporary cosmopolitanism is Immanuel Kant, by outlining the conditions required to establish universal justice. The treaty of Westphalia (1648) had solidified a peaceful system of independent sovereign nation states. Nevertheless, Kant believed that, without an overarching commitment to cosmopolitan legal principles, every sovereign state (and its citizens) would continue to face external threats and that in such a situation it would be unable to

3 "In this avoidable dystopia, the cumulative costs, economic and otherwise, borne by societies will be tremendous". The Cost of Inaction. World Health Organization. (2016). Working for Health and Growth: investing in the health workforce. p.22. https://www.who.int/hrh/com-heeg/reports/en/ 
secure public rights. Kant advocated for the advancement of mutually consistent international and cosmopolitan principles "which may eventually be regulated by public laws, thus bringing the human race nearer and nearer to a cosmopolitan institution". (Brown and Held 2010, p. 8)

In more recent times, scholars such as Thomas Pogge have argued that affluent Western nation states are harming the global poor, including in relation to access to health care, if and insofar as we collaborate in imposing an unjust global institutional order upon them. (Pogge 2005b) He makes the moral case that an institutional order is unjust if and insofar as it foreseeably perpetuates large-scale human rights deficits that would be reasonably avoidable through feasible institutional modifications. Pogge focuses on the design that the global institutional order plays in the persistence of severe poverty. Major economic theory holds that national differences in development trajectories and an 'explanatory nationalism' are the key factors why there is a persistence of severe poverty. (Pogge 2005b) Pogge argues, however, that there are many global institutional factors relevant to the persistence of severe poverty, e.g. through the insistence in World Trade Organization (WTO) negotiations by affluent countries on continued and asymmetrical protections of their markets. Similarly, those rich states also insist that their intellectual property rights, including on seeds, drugs, and diagnostics, must be vigorously enforced in poor countries. At the same time, rich countries pay nothing for externalities such as global pollution and resource depletion to which they are (vastly) disproportionally contributing. Moreover, Pogge states that resource transfers from rich countries keep undemocratic rulers of resource-rich countries in power. Severe poverty in many places is fuelled by local misrule but this is fuelled at the same time by global rules imposed by the richer countries. There is a causal relationship between our global institutional unjust order and the persistence of severe poverty, including the imposition of this order by a small elite faction mainly in rich countries. (Pogge 2005b)

David Held's analysis holds that the paradox of our times exists in the fact there are so many collective issues we must grapple with, moreover, of growing extensiveness and intensity, and yet the means for addressing these are weak and incomplete. He elaborates on policy proposals to improve social-democratic globalization and a human security approach as possibilities to strengthen global governance. To him, this would imply a focus on promoting coordinated state action to tackle common problems, reinforcing international institutions to function effectively, developing multilateral rules and procedures that integrate small and major powers into a multilateral framework. In essence, he argues for a move from (neo)liberal to social democratic globalization, away from policy prescriptions known as the Washington 
Consensus ${ }^{4}$, with at the core the need to connect the security and human rights agenda. For people in the 'majority' (developing) world it is not acceptable to address security problems such as terrorism and human trafficking in isolation unless these issues are connected with fundamental humanitarian issues rooted in social and economic wellbeing such as basic education, access to health care, and clean water. (Held 2006)

Although there are many arguments why such normative cosmopolitanism is relevant for global governance and the advancement of common global health issues, there may be also another analytical, more realist, lens applied for addressing the global health (care) problematic. This approach is described as the cosmopolitan outlook.

\subsubsection{A cosmopolitan outlook}

Ulrich Beck provides an analysis of modern-day global interdependence that he describes as a 'world risk society'. In such a Globalized Risk Society, three axes of conflict are envisaged, being the ecological, economic, and terrorist interdependency crises, respectively. This climate of heightened global threats creates an unavoidable pressure to cooperate. "The conceptualization of threats on cosmopolitan scale creates a shared space of responsibility and agency bridgingnationalfrontiers and divide. This mightlead to cosmopolitan norms and agreements, hence to an institutionalized cosmopolitanism... however existing research on the emergence of corresponding supra- and transnational-organizations and regimes has shown how difficult it is to make the transition from agreements on the threats to agreement on what (institutionalized) form the response should take." (Beck 2006a, p. 23) Beck describes that societies are transiting from a first to a second modernity, in which the main goal of states is no longer governing industrialization, economic growth, and development, but rather tackling the very success of modernity, i.e. the produced man-made risks (e.g. ecological, economic, inequities) on a global scale as a byproduct of modernity. The second modernity can be considered a reflexive modernity. Reflexive modernity is less concerned with expanding the resource base (e.g. in this study the global quantity of the HWF) than with re-evaluating that which is already being used by society. Reflexive modernity uses concepts such as sustainability and the precautionary principle. Progress in the new modernity is

4 The Washington Consensus is a set of 10 economic policy prescriptions developed in the late 80 's. It is considered to constitute the "standard" reform package promoted for crisis-hit developing countries by Washington, D.C.-based institutions such as the International Monetary Fund (IMF), World Bank and United States Department of the Treasury. The prescriptions included policies in such areas as macroeconomic stabilization, economic opening with respect to both trade and investment, and the expansion of market forces within the domestic economy. (Williamson, J. What Washington means by policy reform. Latin American adjustment: How much has happened, 1, 90120. 1990) 
achieved through reorganization and 'reform', amongst others of the governance mechanisms for global challenges. The national outlook and focus are eroding, not disappearing, and will be absorbed in a 'realistic' cosmopolitanism, where other actors (civil society, supranational institutions, transnational corporations, social movements) play an increasingly important role. (Beck 2006a, pp. 72-96)

\subsubsection{A cosmopolitan outlook on health workforce migration}

The inequity seen in international HWF migration is one of these global challenges that can be then considered a 'byproduct of modernity.' Labonté and Ruckert provide three quick 'take-home messages' about the health implications of health worker migration: "It is a global phenomenon, in a context of an overall and increasing shortage; It is not a new phenomenon, but its flow pattern has shifted in recent decades; and health workers save lives, especially in low-income country contexts facing high-burden of disease." (Labonté and Ruckert 2019, p. 194) Health workforce migration is a 'wicked' problem and the global political economy and human rights ethics of this phenomenon are complex. The causes and consequences of HWF migration are multiple and depending on the countries, actors and contexts can deepen or possibly undo existing health inequities. Highly-skilled health workers might leave (already) fragile, understaffed, health systems in countries of origin such as the Philippines or India. However, health care labor abroad also generates considerable remittances that benefit families of the migrant worker in the country of origin and could potentially, via taxations and other channels, reinforce the health systems in the source country. Although it is frequently argued that remittances compensate for the economic losses associated with health labor migration, it remains unclear in most cases who bears the losses and who benefits from the gain in the long term. (Labonté and Ruckert 2019, pp. 200-01)

Over the years, several mitigating policies have been pursued to balance the benefits and losses of HWF migration; allowing temporary movements of health workers via bilateral agreements is one such approach. Germany is attempting this avenue now, under the name 'Triple Win nurses', with a range of countries, notably the Philippines. (Van de Pas and Mans 2019) Another approach is 'bonding' whereby there is a kind of return on a service contract to work in underserved regions in the countries of origin and as part of circular migration promotion. The sustainability of such approaches is questionable, as labor migration has proven to be difficult to 'manage'. There is no enforceable global agreement governing HRH migration apart from services trade agreements that might include consideration of temporary workforce mobility. The main, non-binding, international agreement is the wHO's Code of Practice on the international recruitment of health personnel (see later for details). Recently, Global Skills Partnerships (GSPs) have been proposed. 
"GSP are an exchange of finance and technology for training in the country of origin before migration of potential migrants in exchange for service at the destination. Well-designed partnerships would eliminate and even reverse fiscal drain from origin countries due to new migration, while preserving workers' mobility and providing needed skills at the destination. These partnerships take a dual economic opportunity and turn it into an engine of human capital creation for both origin countries and destination". (Clemens 2015) Nevertheless, there is no evidence that GSPS provide equitable and sustainable solutions in strengthening health systems in both source and destination countries. (Van de Pas and Mans 2019)

Regardless, scholars have provided an ethical defense for restitution (compensation) of the loss of health workers that lies in theories of relational justice. These hold that historic inequities within and between countries are not accidental and are the effect of the past and present socio-political choices that disproportionally benefit wealthier countries. This implies that there could be a moral duty for countries or individuals to provide remedial assistance in overcoming health system inequities, including the related financial and human resources required. (Pogge 2004)

A critical question here is whether a skilled HWF could be considered a Global Public Good for Health (GPGH) ${ }^{5}$ Smith and Woodward argue that while health services are not a 'pure' GPGH (such as some communicable disease control or environmental preservation) they might be considered key 'access goods'. Inadequate health systems limit the ability of populations to benefit from GPGH. "Support to the development or restoration of adequate health services in countries where they do not currently exist may therefore be justified on GPG [Global Public Good] grounds, particularly where universal or near-universal coverage is necessary to ensure GPGH production, as in the case of disease eradication. This represents a strong case for the provision of free health services as a public good at the national level, and for external subsidies to achieve this". (Smith et al. 2003)

Given this definition, HWF development and its international mobility could be considered a GPGH. I argue that such a GPGH approach would then be a cosmopolitan

\footnotetext{
5 Public goods are goods which the "free market" will not provide because they are: nonexcludable: benefits of good available to all; non-rival in consumption: consumption by one person does not prevent consumption by others. The UNDP defines a global public good as "a public good with benefits that are strongly universal in terms of countries, people and generations". Health per se is not a public good. However, the prevention or containment of some communicable diseases may be considered as GPGs. Secondly, another important externality aspect of health amenable to conceptualizing as having GPG properties is that of wider economic externality effects. The economic effects of ill-health on households may be considerable. (Smith, R.D., R. Beaglehole, D. Woodward and N. Drager. 2003. Global public goods for health: health economic and public health perspectives. Oxford: Oxford University Press. )
} 
outlook to address the HWF challenge. I will articulate in the discussion what this would imply for shared responsibilities by countries to invest in and sustain an accessible skilled global HWF.

\subsection{GLOBAL HEALTH POLICY FRAMES}

Regardless of such a cosmopolitan outlook, different foreign and domestic policy approaches-pursued by states and others-shape global health policies and cooperation. Global health cooperation is hence not (only) a matter of science, evidence, and technical solutions but very much so a political domain where international and transnational, socio-cultural, institutional and economic relations provide the structure and values for collaboration and health outcomes. Political tensions, power relations, and different agendas shape global cooperation in the global health domain including cooperation on the best approaches to health systems development and its finance. The several frames or discourses in which global health policies are being proposed provide, sometimes explicitly but often in a more disguised way, the policy framework and underlying norms for global health solutions. These norms and political contestations are often hidden and non-interrogated under a broad definition of global health. (Ooms 2015b) Global health is hence a broad basket term and discipline that can be shaped, categorized and framed according to political needs and values of the main actors involved. Different foreign policy approaches and diplomacy arguments direct priorities and financing in global health programs. Moreover, while these different policy frames might overlap, there are often inherent normative and political tensions between them that are subsumed under 'the global health' umbrella. (Labonte and Gagnon 2010; Lencucha 2013; McInnes et al. 2012; Steurs et al. 2018; Stuckler and McKee 2008) For instance, Labonté and Gagnon deconstruct global health policy drivers into 6 'frames': 'security'; 'economic and social development'; 'trade'; 'Global public goods'; 'human rights' and 'moral/ethical reasoning'. (Labonté and Gagnon 2010) Stuckler and McKee use the metaphors 'foreign policy'; 'security'; 'charity'; 'investment' to describe the policy imperatives of global health programs. (Stuckler and McKee 2008) Lencucha uses the typologies 'Isolationism'; 'charity'; 'security', and 'cosmopolitanism' to describe different ethical perspectives of foreign health policy pursued by states. (Lencucha 2013) While one could discuss semantics and the relative overlap between several frames, the most important aspect is that these different policy choices (and underlying values) have a considerable impact on the conceptual, scientific, and programmatic approaches in global health and their implementation, including for the HWF. While cosmopolitanism and the outlook described earlier focus on human rights and moral imperatives (but not exclusively!), a majority of nation state governments pursue economic (development) or security 
objectives when engaging with other countries including in the domain of global health. I hence need to describe these policy drivers in relation to global HWF development. In this thesis I will analyze the 'economic' and 'security' frames in global HWF development from a political-economy approach. This follows the need 'to be more critical about the role of power in global health-who wields it and how it is utilized to privilege certain meanings and roles (and exclude others) and why particular actors are able to exert legitimacy to define problems and set the global health agenda". (Birn et al. 2017, p. xxii) This also provides clarity on if and why there is an inherent or hidden tension between different ideologies and approaches in developing the HWF.

\subsection{HEALTH EQUITY IN A GLOBALIZING ERA OF DEEP ECONOMIC INTEGRATION}

Labonté and Ruckert write that there is a cosmopolitan 'hopefulness' implied by globalization processes, as these could represent 'a single world society' marking the onset of a 'borderless world'. They suggest that this hopefulness, however, may also be hopelessly naïve, in the sense that there is no world government or system of global citizenship rights and responsibilities by which a global society might be formed. (Labonté and Ruckert 2019, p. 2) There are several definitions of globalization but the economic angle stands out. Class distinctions are the impact of a disequalizing global economy. Globalization can be summed up by 'the triumph of a capitalist world economy tied together by a global division of labor'. (Labonté and Ruckert 2019, p. 3) Bauman already noted that the world is increasingly divided into two classes of citizens. The first class, he calls the privileged 'Tourists' which are mobile and with money and status. The second, he calls 'Vagabonds'. Those are the less privileged billions whose migrations to escape conflict, poverty or climate breakdown are increasingly unwelcome. (Bauman 1998) As economic globalization has been the driving force behind the overall process of globalization over the last two decades, it is these economic policies and practices that require the closest scrutiny from a health vantage. (Labonté and Ruckert 2019, p. 4)

The same authors also make the compelling argument that globalization itself is an important Structural Determinant of Health (SDH) and thus deserves its own analysis of how it affects health outcomes. The wHO's Commission on Social Determinants of Health (CSDH) was tasked in 2008 to explore the relationship between economics and social policy-making, its underlying politics and how it affects health outcomes, specifically equity in health. The CSDH commissioned nine knowledge networks to answer that question. One of these was the Globalization Knowledge Network (GKN) that eventually completed a meta-synthesis on globalization and its impact on the social determinants of health, including a conceptual framework. (Labonté et al. 
2007) One of the arguments advanced by the synthesis study and framework is that one cannot envision policy or programmatic solutions to health inequities without working back and around the pathways linked to globalization. The GKN concluded that it is vital to understand globalization as a 'determinant of social determinants of health' within national boundaries as well as underscore that the links between the globalization process and health outcomes are not necessarily straightforward. (Labonté et al. 2007)

There is a fierce and ongoing debate whether globalization has been, or can be, good or bad for health. This thesis provides a humble attempt to contribute to this debate as well. There is more consensus, however, on the fact that neoliberal economic policies since the 80 s have had a negative impact on health systems development and social protection policies in several countries. Labonté and Ruckert suggest that we are currently in 'phase 3.0 of Neoliberalism' with austerity becoming a structural economic adjustment policy not only in LMICs but also in High-Income Countries (HICs) such as Germany, Australia, and Canada. The continued focus on a further reduction of public spending and public sector management seems unwarranted if one realizes that there is a limited need for further liberalization and that in many countries there is sound fiscal management. However, researchers suggest that by 2020 over $80 \%$ of the world's population will be living under the yoke of fiscal contraction, with global gross domestic product (GDP) 5.5\% lower in 2020 than it would have been without the austerity agenda. (Ortiz et al. 2015) It is against this background of enduring global austerity that this study looks into the possibilities and challenges of developing the HWF from a global health perspective. Despite all the policy alternatives and growing criticism, neoliberalism has proven to be resilient, even ten years after the Great Financial Crisis of 2008. In as much as technical, fiscal and public health sound policy measures can be constructed to improve health employment, it will depend on the political, legal, and ethical drivers to enable such a space. In our globalized societies, the question is what the actual polity is, or could be, to devise social policies that address transnational challenges. (Labonté and Ruckert 2019, p. 65)

\subsubsection{The health labor market}

Aclassical economic discourse analyzes the workforce from a labor market viewpoint. From such a perspective, it is mainly the demand for and supply of health workers that shapes inequalities in distribution. Regulatory interventions by the state could mitigate imbalances. In this view, it is mainly economic growth at national, and at decentralized levels, that can spur investment in the workforce, as demand for health services and care will grow. (Labonté and Ruckert 2019, pp. 164-91) Macroeconomic policies have effects on fiscal space within national government budgets 
to recruit and deploy health workers. Fiscal capacity related to the economic performance and restrictions on public spending imposed by the Bretton WoodsInstitutions, consisting of the World Bank (WB) and International Monetary Fund (IMF), on indebted nations have led to reduced capacities to integrate health care workers into the workforce of the private and public sector. An analysis by Stubbs and colleagues concluded that such economic conditionalities impeded progress toward the attainment of UHC in 16 West African Countries in the period from 1995-2014. (Stubbs et al. 2017)

The WHO has over the years also made a shift in its approach towards HWF development by making a more explicit link with job creation and economic growth. Likewise, the wHO has become increasingly engaged in the political-economy analysis for health but focuses rather exclusively on processes within countries (Reich 2019), thereby neglecting economic interactions between countries and transnational phenomena such as labor mobility. In 2016, the United Nations (UN) SecretaryGeneral set up an inter-sectoral High-Level Commission on Health Employment and Economic Growth drawing a diversity of experts from the education, finance, health and labor sectors. Their task was to identify strategies to avert a projected shortfall of 18 million health workers by 2030-primarily in LMICs-and guide action on the unprecedented global demand for health and social sector jobs in wealthier economies. This Commission found evidence that investing in the HWF is a driver of inclusive economic growth, dispelling perceptions of health as a consumptive cost. In its report 'Working for Health and Growth', the Commission made ten recommendations with five immediate actions to expand and transform the HWF. The five actions to "expanding and transforming the health and social workforce at country level" are the following: Concerted tripartite social dialogue; Improved health labor market data, analysis, and evidence; Enhanced national workforce strategies; Sustainable domestic and international investment; Transformation and scale-up of education, skills, and decent job creation. (wHO et al. 2017) While this Keynesian economic stimulation approach to health and development is laudable, it does put the onus on investing in the HWF at the country level. This puts aside the question of whether countries have the actual fiscal policy space and finance to pursue health investments. It likewise neglects historical responsibilities and moral obligations by wealthy countries to invest in health systems abroad. (Pogge 2004)

\subsection{GLOBAL HEALTH SECURITY AND THE SECURITIZATION OF HEALTH}

Health protection has always been a core policy driver for public health authorities around the world and likewise for the wHO. A focus on the prevention and control of infectious disease outbreaks is at the heart of this approach. The World Health 
Report from 2007 explicitly started using the term 'Health Security'. (World Health Organization 2007) In the report, 'global public health security is defined as the activities required, both proactive and reactive, to minimize vulnerability to acute public health events that endanger the collective health of populations living across geographical regions and international boundaries.' (World Health Organization 2007, p. ix) The report asserts that over 57 countries, due to workforce shortages, are struggling to provide even basic health security to their populations. (World Health Organization 2007, p. 57) It must be noted that the term 'health security' has caused controversy, with Aldis noting that there is an incompatible understanding of the concept between developed and developing countries and that behind this tension lies a fear of hidden national security agendas. (Aldis 2008) Nevertheless, a decade later Global Health Security (GHS) has become an established domain for research, policy, and practice. The Sydney Statement on Global Health Security (2019) states that achieving GHS is intrinsically linked in efforts to achieve UHC, strengthen other vital aspects of broader health and security systems, and the SDGs. (Global Health Security 2019) It also states that countries with higher capacity to respond to adverse public health events have a moral duty to work in partnership with those with lower capacity to strengthen their capabilities in a sustainable manner. (Global Health Security 2019) Interestingly, in most of the literature on GHS, there is no (or only indirect) reference to the need to invest in the workforce. The workforce seems a 'static' and utilitarian asset that will be available to respond to and address public health threats when required. The 2014-2015 Ebola Virus Disease (EVD) outbreak, and other infectious outbreaks, provided insight to the vulnerability of health systems in many LMICs, mainly due to a weak and dysfunctional HWF. (The Lancet - Editorial 2016) This provided for a political momentum to invest in strong and resilient health systems. (Kieny and Dovlo 2015) The wHO in its program 'Working for Health' consecutively put investing in the International Health Regulations ${ }^{6}$ as one of the main priorities, including skills development of national and international health workers in humanitarian settings and public health emergencies, both acute and protracted. (wHO et al. 2017) It remains to be seen whether the 'security' frame offers sufficient traction in providing long-term investments in the workforce. There remains criticism about the way in which a predominantly North American and European interpretation of risk and susceptibility has been used to define health security discourse internationally. (Smith 2015) Rushton provides the insight

\footnotetext{
6 The International Health Regulations (IHR) are an international legal instrument that are binding on 196 countries across the globe, including all the Member States of the WHO. Their aim is to help the international community prevent and respond to acute public health risks that have the potential to cross borders and threaten people worldwide. The IHR, which entered into force on 15 June 2007, require countries to report certain disease outbreaks and public health events to the WHO. The IHR define the rights and obligations of countries to report public health events, and establish a number of procedures that the WHO must follow in its work to uphold global public health security. (WHO, 2005 https://www.who.int/ihr/about/en/)
} 
that two radically different formulations of health security can be distinguished. There is a statist/national security formulation, which takes the state as its referent object and is focused primarily on diseases entering or otherwise destabilizing states and societies. In contrast, there is a globalist/human security understanding, which takes the individual as the referent object and is open to the consideration of a much broader range of issues that threaten individual health and well-being. (Rushton 2011) While the latter approach, also known as a human security approach? would come closest to the cosmopolitan outlook, it seems that statist security considerations are dominant in contemporary global health policy and practice. (Global Health Security 2019)

\subsection{GLOBAL HEALTH GOVERNANCE}

The sections above provide the insight that global health policy and programs, including for HWF development, are subject to underlying political tensions and normative drivers that shape the eventual direction of health cooperation between actors. Kickbusch argues for strengthening global health diplomacy and GHG as a means to mitigate and align different policy approaches. Health is on the radar of foreign policy because it has become integral to the three global security, economic, and social justice agendas. There is a need for public health practitioners to work more closely with foreign policymakers. (Kickbusch 2011) In Kickbusch's vision, global health is essentially "characterized by new multi-actor approaches that aim to deal with global interdependence as well as new power relationships". It is then required to develop a global governance regime that has a "purposive order for the management of interdependence in the absence of a global state”. (Kickbusch and Szabo 2014) While global health governance, global health systems, and its challenges can be categorized in different ways, (Frenk and Moon 2013; Lee and Kamradt-Scott 2014) Kickbusch suggests that it can prove helpful to analyze global health governance along three political spaces: global health governance, global governance for health and governance for global health. (Kickbusch and Szabo 2014)

Global health governance refers mainly to those institutions and processes of governance which are related to an explicit health mandate, such as the wHO; Global governance for health refers mainly to those institutions and processes of global governance which have a direct and indirect health impact, such as the UN, WTO or

7 The human security approach was introduced in the 1994 global Human Development Report (HDR). The human security approach broadens the scope of security analysis and policy from territorial security to the security of people. The $1994 \mathrm{HDR}$ highlighted two major components of human security: 'freedom from fear' and 'freedom from want'. (UNDP. Human Development Report 1994: New dimensions of human security) 
the Human Rights Council (HRC); Governance for global health refers to the institutions and mechanisms established at the national and regional level to contribute to GHG and/or to governance for global health such as national global health strategies or regional strategies for global health. "In all three political spaces, the involvement of a multitude of state and non-state actors has become the norm; that is why issues of legitimacy, accountability and transparency have moved to the fore." (Kickbusch and Szabo 2014)

This GHG framework is depicted as follows:

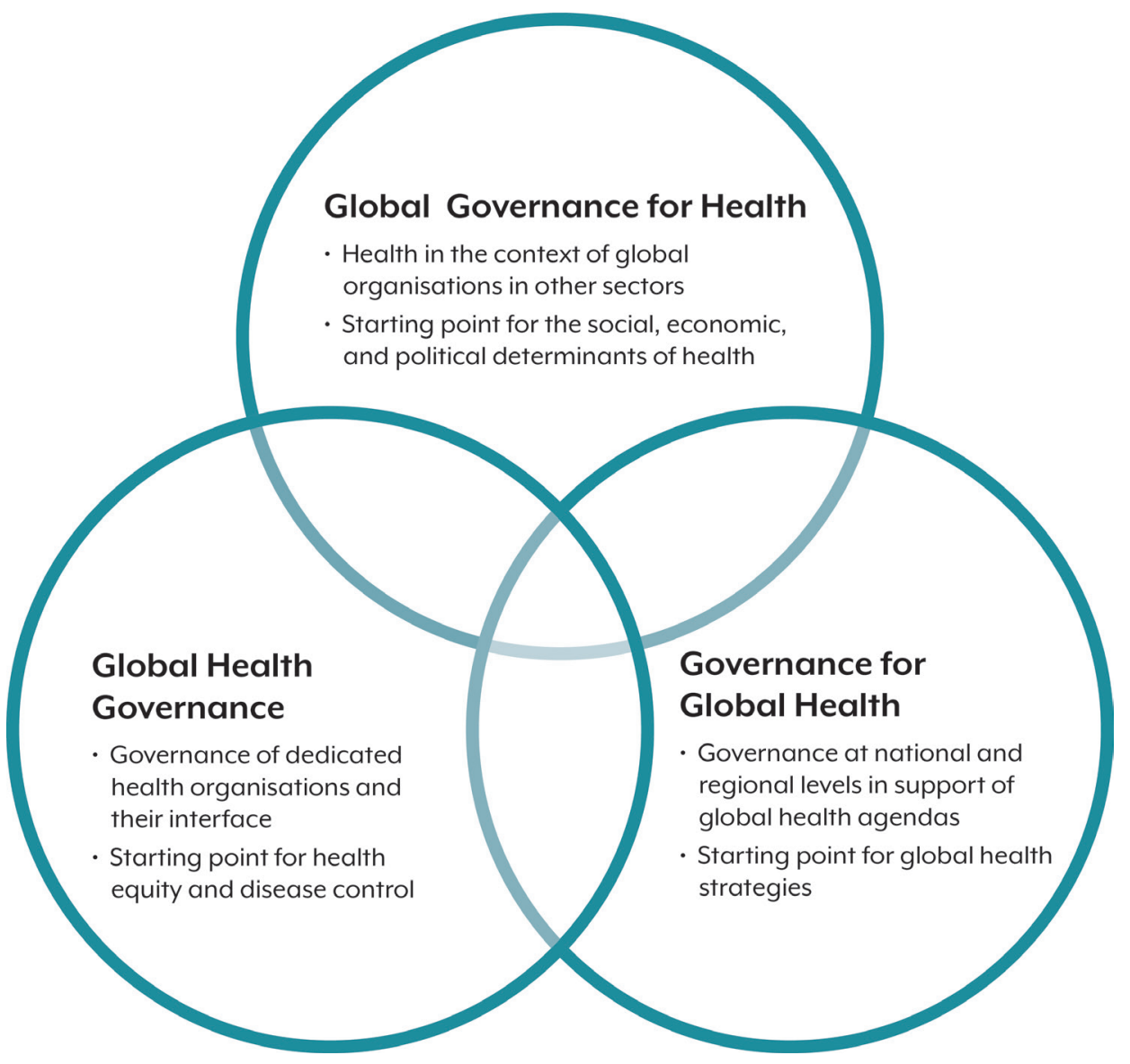

Figure 1.1: Global Health Governance along three political spaces

According to these authors, GHG challenges overlap and it is essential that GHG institutions firmly establish processes to link actors within and between dimensions. "The collective problem solving required in the global public health domain requires controversial actors to be involved but without a commonly agreed rule-based system for including non-state 
actors in global governance institutions, it is difficult to subject powerful organizations, large corporations, foundations and NGOs [non-governmental organizations] to critical analysis." (Kickbusch and Szabo 2014)

This vision of global health development can be regarded as a form of complex multilateralism. Despite all challenges and the gridlock of certain governance regimes, global health is a complex adaptive system that will find solutions and pathways to address pressing global health problems. (Hill 2011)

\subsubsection{Globalization paradox}

I challenge the global health diplomacy and global governance approach as advanced in the former section. Global health diplomacy, including its HWF components, might not be so 'revolutionary' and inclusive as its proponents argue it to be. Perhaps it might even be a remediation with or regression from older approaches of publicand international health. (Labonté and Gagnon 2010) I criticize the 'integrative' potential of GHG based on a theory known as 'the Globalization Paradox' as advanced by the economist Dani Rodrik. (Rodrik 2011a) In this work, he brings forward the thesis that there is 'a political trilemma of the world economy'. The trilemma exists in the fact that there is a fundamental tension between national democratic space and global markets. There are three options to manage this tension. Democracy can be restricted in the interest of minimizing international transaction costs, disregarding the economic and social havoc that the global economy occasionally produces. Globalization can be limited in the hope of building democratic legitimacy at home. Alternatively, democracy can be globalized at the cost of national sovereignty. This is, in essence, a menu of options for reconstructing the world economy. (Rodrik 201la, pp. 106-18) This is visualized as follows:

The Political Trilemma of the World Economy

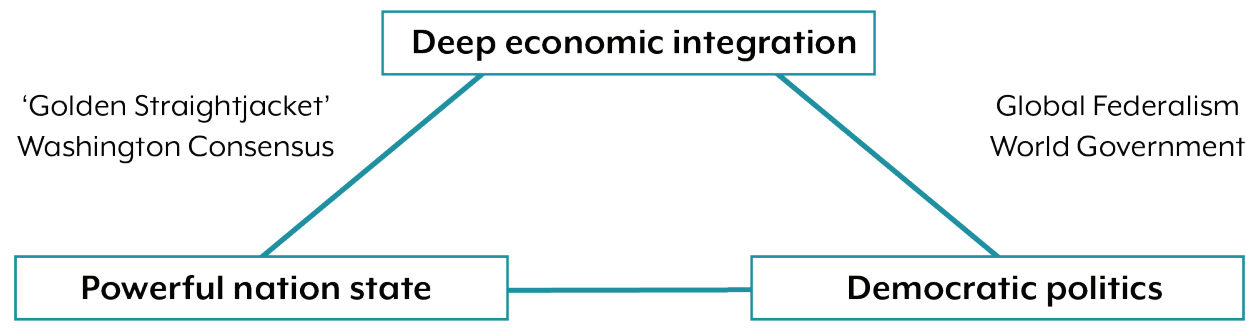

'Embedded liberalism'

Bretton Woods compromise

Figure 1.2: The Political trilemma of the World Economy 
Rodrik argues that we cannot have hyperglobalization, democracy, and national self-determination all at once; we can have at most two out of three at the same time. This theory is basically an anti-thesis to the GHG framework in Figure 1.1. The GHG approach takes it in essence for granted that (deep) economic integration (also known as hyperglobalization), powerful nation states and democratically legitimate policies could co-exist. There are inherent domestic and international political drivers that shape the outcome of this trilemma, and hence also the policy space of global health actions, which too often are dictated by the interests of nation states and other powerful actors in our globalized, integrated economies. Democratic principles, in both global health and other policy areas, are now under considerable pressure in both LMICs as well as European and other HICs. (Diamond 2015)

The political trilemma and the three different sides of its triangle follow in essence different streams or 'images' in international relations theory. They also mimic the different policy imperatives or 'frames' outlined before. 'Golden straightjacket' is a term coined by Thomas Friedman and describes the neoliberal policy dictates in an integrated economy. "Asyour country puts on the Golden Straitjacket, two things tend to happen:your economy grows andyour politics shrink... Onceyour country puts it on, its political choices get reduced to Pepsi or Coke." (Friedman 2000) This view is partly a (neo)realist approach in international relations where nation states via economic, security and other means aim to maintain their own interests (above other states and potentially also their own citizens). There is a presumed balance of power between states, as principal actors in an anarchical world, lacking a legitimate global government. The golden straightjacket also embodies an economic structuralism approach that sees capitalism as the key historical factor and defining characteristic of the system as a whole. (Kauppi and Viotti 2009) The irony exists in the fact that neoliberal policies (initiated by the United States (US) and European countries) since the 80s have provided so much space for powerful financial non-state-actors such as investment banks, transnational corporations, insurance companies, etc., that countries now have huge difficulty to regulate them. The genie is out of the bottle.

The 'embedded liberalism' approach and Bretton Woods compromise is a liberal approach to international cooperation which recognizes that there is interdependence in a globalized world in which states, international organizations and non-governmental organizations (NGOS), multinational corporations, interests groups, and individuals operate in complex arrays of overlapping or cross-cutting coalitions and networks. The Bretton Woods compromise builds on the agreement with the same name from 1944 that recognized that there should be a regulated international monetary system, based on Keynesian economic principles, that protects and recognizes that countries have rights and responsibilities vis-à-vis each other in an interconnected world. This approach can also be regarded as a complex 
multilateralism approach in which there is slow, adaptive governance development by international institutions, such as the UN and the wHO and global public-private partnerships, dealing with health and other challenges. (Kauppi and Viotti 2009) This is, in essence, the de-facto modality of many global health policies and actors dealing with (global) governance issues and being taken for granted as the normal state of affairs.

The last side of the triangle, global federalism, is a normative image of what global health could be in a globalized economy based on democratic principles and possibly facilitating health equity beyond the nation state. This normative approach is regarded as being based on universal or cosmopolitan norms and values. This could be a Kantian approach to human dignity or a more utilitarian global public goods approach. In addition, there is a question of whether conceptions of justice and human rights have boundaries limiting them to particular societies or cultures? Or is justice by nature universal and cosmopolitan? Social contract theory begins with a domestic question of justice and then aims to expand these normative concerns to international politics. In practice, of course, we face enormous obstacles trying to apply these cosmopolitan ethics as the basis for constructing a radically new and just world, given the present division of the world into separate, sovereign states with very different perspectives. (Kauppi and Viotti 2009, pp. 407-08) It is here that the cosmopolitan outlook becomes an interesting angle to imagine new political perspectives as it conceptualizes threats and global risks on a cosmopolitan scale and creates a shared space of responsibility and agency bridging national frontiers and divides. (Beck 2006a)

I have outlined in the above section that different imperatives shape foreign policy and global health cooperation, including in the field of HWF cooperation. Notably, there may be security, economic, and human rights agendas and tensions driving priorities for investments and policies in the global health domain. The cosmopolitan outlook (Beck 2006a) based on a realistic cosmopolitanism and reflexive modernity approach can be considered a contemporary approach to address global health risks and economic externalities faced by globalized societies. I have described how global health diplomacy and GHG is conceptualized along three political spaces. Lastly, by invoking the thesis of the globalization paradox and its political trilemma, I aim to demonstrate that there is an intrinsic tension in the contemporary world economy. This tension could possibly also explain why outcomes in the global health domain, including for advancing UHC and HWF have been below expectations. The following section will describe how global health governance and the political trilemma impact international HWF policy development. Could a cosmopolitan outlook overcome this tension? 


\subsubsection{Global health workforce governance complexity}

Health workforce development, and health labor markets generally, are mainly approached from a first modernity perspective. This is a national sovereign model of development that follows 'explanatory nationalism' thinking. Countries, whether rich or poor, and regardless of their historical development, are expected to increase their domestic revenues, enhance their fiscal capacity, and plan their health finances advancing UHC and health systems strengthening. The HWF and related wage bill should expand within this national framework. Official Development Assistance (ODA) is considered to have a leveraging and investment function, and direct financial assistance (to pay HWF salaries) is only deemed appropriate in fragile settings and low-income countries when there is a humanitarian or global health crisis (or risk of one). (United Nations General Assembly 2015) For instance, the United Kingdom's (UK's) Department for International Development (DFID) has co-financed public HWF salaries in Malawi from 2005-2008 as a way to address the expanding HIV epidemic but this was considered an exception necessary to fend off a global security threat. (McCoy et al. 2008b) There remains much reluctance in highincome countries to pay for the recurrent costs associated with HWF employment and education in low-income countries. The main thinking behind this is that such external financing (development aid) of public salaries is unreliable and would expand the fiscal space in countries in an unsustainable way. (Heller 2005) I argue for a reflexive modernity to global HWF development instead. This might entail a balanced complex of existing (nation states) institutions together with other and new agencies that set the framework, rules, and governance modalities that guide the development of an increasingly mobile health labor force. Several examples show that this second modernity reality has become relevant for health systems development and not only in LMICs. For instance, health professional mobility is a persistent dynamic of labor markets and health policy within the European Union (EU). It is a fast-moving target resulting in increasing inequalities in HWF distribution especially since the 2008 financial crisis with its related austerity measures. Many health professionals have left Southern and Eastern European countries to start working in 'greener' pastures in Northern and Western Europe. The EU and its Member States have true difficulty governing this mobility and equal distribution because health and social policies remain a national mandate while at the same time the EU has become an open market that facilitates the mobility (and trade) of goods, services, and people. (Buchan et al. 2014)

Concerning GHS, global health lawyer David Fidler claimed in 2003 that Severe Acute Respiratory Syndrome (SARS) was "the first Post-Westphalian pathogen". (Fidler 2003) The Ebola viral disease outbreak in 2014-2015 has been described in similar terms. (Kickbusch and Reddy 2015) While the response to the sARS outbreak has led to a 
revision of the wHO's International Health Regulations (IHR) in 2005, a decade later the implementation of the IHR remains problematic. Recommendations in 2009, in a review of the IHR after the global response to the HIN1 pandemic, already called for setting up a contingency fund and a global emergency workforce to deal with such threats. (World Health Organization 2011b) The Ebola epidemic has shaken up the need for the international community to develop both core HWF capacities and a contingency workforce in LMICs. The WHO relies more and more on its Global Outbreak and Alert Response Network (GOARN) ${ }^{8}$ of non-state actors to provide these resources in the future. Although it is a legally binding treaty, the IHR remains 'soft' law with no framework of sanctions. Member states of the wHO have ignored some of its recommendations such as providing financial assistance to LMICs in order to develop these core capacities. (Moon et al. 2015)

Against a backdrop of globalization, institutional proliferation, and harder problems, HRH governance has increased in complexity. A key challenge is the coordination of responses within the different multilateral organizations that are involved in the multifaceted arena of HRH development and mobility. The main international governance mechanism on HWF migration is the WHO's Code of Practice on the international recruitment of Health Professionals (Global Code) ${ }^{9}$ that was adopted after a six-year negotiation process at the $63^{\text {rd }}$ World Health Assembly in 2010. It is a voluntary, non-binding code, with substantive norms advanced in a rather soft diplomatic way to Member States. The wHO Global Code was never intended to be the final answer or encompass the whole solution to the challenges associated with health worker migration. Rather, the goal of the drafters was to establish a global platform that could provide a framework for continuing dialogue and cooperation among states on what is undoubtedly a topic of significant complexity and sensitivity. (Taylor and Dhillon 2011) The wHO is mandated to report every three years on the implementation of the code by Member States. Nevertheless, over the years, it has become clear that the governance challenges concerning workforce migration demand future arrangements to include involvement with a range of other actors

8 The Global Outbreak and Alert Response Network (GOARN) is a WHO network of over 200 technical institutions and networks globally that respond to acute public health events with the deployment of staff and resources to affected countries. Coordinated by an Operational Support Team based at the WHO headquarters in Geneva and governed by a Steering committee, the GOARN aims to deliver rapid and effective support to prevent and control infectious diseases outbreaks and public health emergencies when requested. (WHO, 2019 https://www.who.int/ihr/ alert_and_response/outbreak-network/en/)

9 The Code aims to establish and promote voluntary principles and practices for the ethical international recruitment of health personnel and to facilitate the strengthening of health systems. Member States should discourage active recruitment of health personnel from developing countries facing critical shortages of health workers. The Code was designed by Member States to serve as a continuous and dynamic framework for global dialogue and cooperation. (WHO, 2010 https://www.who.int/hrh/migration/code/code_en.pdf/) 
and policy domains. This would require, amongst others, policy coherence with the ILO's Multilateral Framework on Labor Migration. It is, in addition, necessary to make HRH and health labor migration an issue within the Sustainable Development Agenda and in the debate on the role of global trade agreements in the quest for development. Moreover, global and regional trade agreements are likely to increase (temporary) labor migration in the health care sector. (Yeates and Pillinger 2013) The WHO has established an International Platform on Health Worker Mobility (IPHWM) to engage in "a new and more nuanced dialogue with states and relevant stakeholders on investments that are inherent in or arise from the international migration of health workers". This multi-stakeholder platform is being co-hosted by the ILO, the Organization for Economic Co-operation and Development (OECD) and the wHO. (World Health Organization 2018b) Given the actors involved in this international platform, there is much attention to the economic and employment aspects of HWF migration.

Several authors have argued from a cosmopolitan perspective for advancing global health governance and developing pathways towards a more shared notion of health development. This includes proposals to advance global social protection models for health (Ooms 2015a); Global constitutionalism as a possibility to improve current global health governance arrangements and address legitimacy deficits (Ooms and Hammonds 2016); Policy proposals on how to overcome the gridlock in global health governance (Labonté and Ruckert 2019, pp. 343-63; Ole Petter Ottersen et al. 2014); Cosmopolitanism as an ethical principle to drive foreign policy for health for and beyond the state. (Lencucha 2013) In contrast to the relative abundance of ideas and proposals on how to advance global health finance and governance from a cosmopolitan and human rights perspective suggestions on HWF development along these lines have been more limited. A notable exception is in the work by Mackey and Liang who assessed and proposed policy proposals for the redistribution of financial resources to address the negative effects of brain drain related to medical migration. They argued for a Global Health Resource fund and governance mechanism that would provide for cost-sharing with and reimbursing of resource-poor countries for brain-drain losses in the health care sector. Such a fund could contribute to HWF development and health systems strengthening in LMICs and at the same time still facilitate HWF migration in a globalizing health labor market. (Mackey and Liang 2012; 2013)

\subsubsection{Gridlock in global cooperation for health}

Despite all the compelling arguments to advance global governance in the domain of health and other regimes, there is contemporary gridlock in global cooperation. The 'Globalization Paradox' theory might explain this from an economic perspective, but political scientists provide complementary analyses. Interdependence has now 
progressed to the point where it has actually altered our ability to engage in further global cooperation. That is, economic and political shifts that are in a large part attributable to the success of the post-war multilateral order are now amongst the very factors grinding that system into gridlock. The need for international cooperation has never been higher. Yet, institutionalized multilateral cooperation has stalled. Gridlock is becoming a general feature of global governance and moving beyond one single issue: cooperation seems to be increasingly difficult and deficient at precisely the time when it is needed most. (Hale et al. 2013b) Kickbusch and Reddy describe how gridlock in global health governance expresses itself. First, due to geopolitical multi-polarity, it has become more difficult for the wHO's 194 Member States to come to a consensus on common policies. Second, there is institutional inertia as reform and financial investment in UN institutions have been limited over the last few decades. Third, there has been fragmentation in global health cooperation due to the proliferation of many new actors and funding channels. Fourth, problems have become harder, with issues such as health systems development, addressing the commercial determinants of health, and environmental degradation, requiring inter-sectoral action across actors and countries. (Kickbusch and Reddy 2015)

The WHO is a clear example of how the gridlock in global health governance paralyzes common action to overcome global health challenges. Its financial autonomy has been severely restricted as $80 \%$ of its budget comes from 'tied' funding for selective programs. This principle is known as Trojan multilateralism in which the (main donor) countries control multilateral institutions in a tight way as to have the organization focus on objectives that are in line with the foreign policy objectives of these countries. (Sridhar and Woods 2013) This financing trend has limited the WHO to pursue work on comprehensive primary health care, health systems strengthening and HWF development. The financial and human resource basis for these programs has been limited while the main donor countries in global health prefer to invest ODA in global health initiatives that implement more 'vertical' programs that aim for relative short-term results. The gridlock of the institution is one of the reasons that the wHO reform initiated in 2011 has been disappointing. Its 'capture' became painfully visible during the response to the Ebola outbreak in West-Africa. (Legge et al. 2017)

From a reflexive modernity perspective, this GHG model is not fit for purpose anymore in the $21^{\text {st }}$ century. The requirements to build GPGH and to reduce global health inequities compel researchers, practitioners, and policymakers to consider pathways and options to overcome the gridlock that we are currently facing. The globalizing labor market for health workers is not the only issue that requires governance and mitigation for its outcome to be social and democratic. There is also a need for a shared responsibility mechanisms (including co-financing) to 
deal with global public 'bads' in health. This raises questions such as: 1. Could an international workforce be available to address similar epidemics like the 20142015 Ebola outbreak in the future? 2. How to finance the 18 million health workers needed to close the gap required to advance UHC and meet the SDGs by 2030 ? 3. Does the current impact of climate change on health outcomes across countries facilitate an international mobilization of, and advocacy by, health workers? 4 . What are the workforce requirements to address the health needs of the international refugee population? Although there are political agreements on humanitarian assistance there is (not) yet an institutionalized international agreement, matched by an international financial framework, to develop and strengthen health systems to prevent and prepare for health emergencies. (Gostin et al. 2015; Hill et al. 2011)

\subsection{RESEARGH QUESTIONS}

The tensions in GHG, aggravated by the globalization paradox and its political trilemma, provide an intrinsic challenge and complexity for HWF development at the domestic, international, and even transnational level. This study aims to advance the ethical, public health, governance, and political perspectives to enhance the shared responsibilities and co-financing of HWF development and its institutions from a cosmopolitan outlook. Would such a cosmopolitan approach be possible and legitimate, while actually improving workforce availability and health equity? The main research question of this thesis is formulated as follows:

Can global health policies rooted in a cosmopolitan outlook advance health workforce development in an equitable manner?

This overall question is deconstructed and analyzed via four specific research areas:

1. Do governments in countries with HRH challenges have the policy space to expand and reform the workforce?

2. Do modern global health institutions and policies have the democratic legitimacy and space for health workforce development?

3. How do policies of governance regimes outside the health domain impact the health workforce?

4. Can cosmopolitan health policies overcome the gridlock in global cooperation for health workforce development? 
I have categorized three research areas following the GHG framework of three political spaces. (Kickbusch and Szabo 2014) The fourth research area, on the space for cosmopolitan health policy, provides a reflection on the globalization paradox and its political trilemma. (Rodrik 2011a)

\subsection{CONGEPTUALIZING GLOBAL HEALTH WORKFORGE GOVERNANCE AND ITS PARADOX}

I argue that global HWF development and governance is not only shaped along the three political spaces following the GHG conceptualization depicted in Figure 1.1 (Kickbusch and Szabo 2014); it is also impacted by the policy tractions in the 'political trilemma' depicted in Figure 1.2. (Rodrik 2011a) I propose to bring these two frameworks conceptually together to advance the thinking on a cosmopolitan outlook for HWF development. Such a framework would capture the need to work along the three political spaces and at the same time acknowledge that there is an inherent tension, the trilemma, to advance global HWF development in an equitable manner. Such a conceptualization outlines the policy space as well as the limitations in moving the global HWF agenda forward.

At the beginning of the thesis trajectory, a detailed mind map on the HRH challenge was produced that follows the three political lines of action in GHG. This conceptualization has been combined with the political trilemma challenge into one framework and provides the core structure for the thesis (Figure 1.3). 


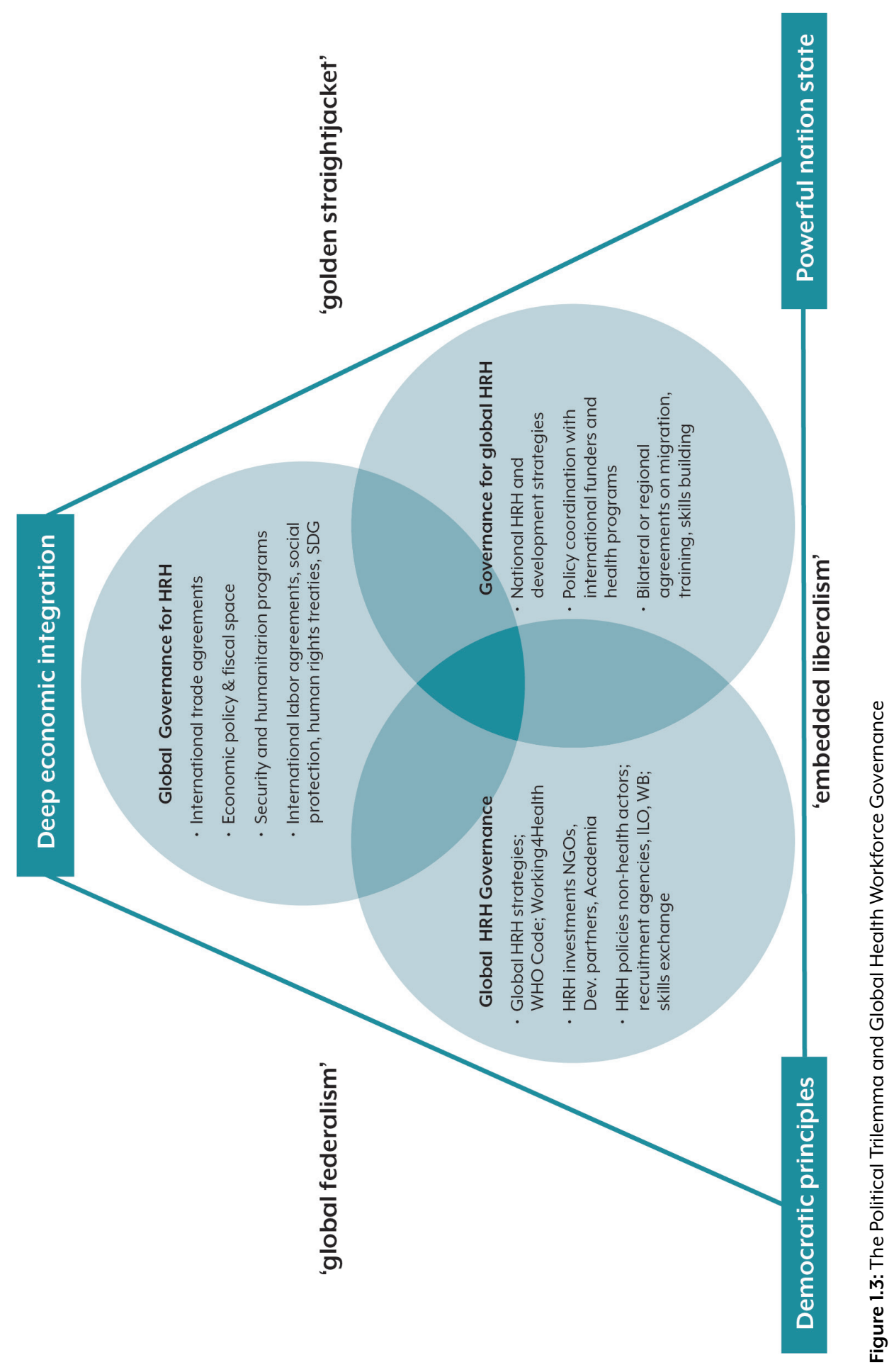


The three circles of this Venn diagram provide an overlapping overview of the three governance spaces that matter for Global Health Workforce (referred to as Human Resources for Health, HRH) governance and its development.

Governance for global HRH takes place at the national or regional level. This could include the development of national HRH strategies, including policy actions such as enhancing education, employment, retention, labor market analysis, community health workers development, skills-mix optimization and improving HWF accounts and information systems. This has the aim to contribute to global health strategies such as UHC, implementation of the SDG, IHR, Global Strategy for Women's, Children's and Adolescents' Health ${ }^{10}$, etc. This is the national space where coordination needs to be pursued with multiple international actors, such as non-governmental organizations (NGOs), un agencies, the World Bank as well as private service providers on the alignment of their programs with a national HRH strategy. Lastly, bilateral or regional agreements can be developed on the education, mobility, and recruitment of health workers across borders and requires cross-sectoral governance.

Global HRH governance pertains to the role of multilateral or international health organizations in developing policies strengthening the workforce. There is a main role here for the wHO as a key actor having adopted the Global Strategy on HRH: Workforce 2030 and leading the implementation of the interagency five-year action plan on health employment and economic growth. (wHO et al. 2017) This links HWF development to broader SDG objectives such as UHC, gender equality, decent work, economic growth, and the reduction of income inequalities. The wHO is also the host of the Global Code and its related IPHWM. Nevertheless, there are many international actors working on HWF development. These include NGOs and many others financing community health worker programs. Other relevant actors include global public-private partnerships for health (Global Health Initiatives such as the Global Fund (GF) and Gavi-the vaccine alliance), bilateral development agencies, and not to forget the many bilateral partnerships between educational and scientific institutions in skills building. There are a few international platforms and networks where collaboration and a common agenda between those actors are pursued. The Global Health Workforce Network (GHWN), the successor of the Global Health

10 The Global Strategy (2016-2030) is a roadmap to achieve the right to the highest attainable standard of health for all women, children, and adolescents. The new Strategy-updated through a process of collaboration with stakeholders led by the WHO-builds on the success of the 2010 Strategy and its Every Woman Every Child movement. It is a platform which puts women, children, and adolescents at the heart of the UN Sustainable Development Goals. (WHO, 2016 https://www. everywomaneverychild.org/global-strategy/) 
Workforce Alliance, is one of these ${ }^{11}$. The GHWN and the wHO are hosting a multiactor Global Forum on HRH every 3 years. Research and a common policy agenda towards HRH development are pursued during these fora ${ }^{12}$. It is noteworthy that multilateral agencies that do not have an original health mandate are now actively involved in HWF skills development programs, such as the World Bank. (Remcovan de Pas and Mans 2019) The ILO's promotion of decent employment in the health sector contributes a considerable part of its agenda on the future of work. (International Labour Organisation 2019) The wHO's 'Working for Health' action plan and the IPHWM provide the spaces where alignment on HRH policies could be pursued between those multilateral institutions and Member States. (wHO et al. 2017)

Global governance for HRH would then include the need to discuss HWF issues and policy requirements to be addressed with organizations across other policy terrains. Notably, the need to seek a common approach in fiscal policies development and wage bill arrangements while negotiating economic packages and reforms (financial investments, loans) between Member States and international financial institutions such as the IMF can have a profound impact on the sustainability and policy space to develop a strong workforce. (Alexander E Kentikelenis 2017) Although in the past, health services have been excluded from many trade agreements there are indications that this international 'care market' might liberalize further in the coming years. This would have an impact on the medical mobility of patients, health workers and the outsourcing of services. In the EU with its open labor market, this is already well visible. (Buchan et al. 2014) Close monitoring of and engagement by health experts in the inclusion of future health services in trade agreements would be needed to avoid any negative impacts on HRH development. (Missoni 2013)

The recent killing of an epidemiologist employed by the wHO, as well as other health care workers in trying to curb the Ebola epidemic in the eastern part of the Democratic Republic of the Congo (DRC) indicates the impact of security policies, or the lack thereof, in relation to violence directed towards health workers. (World Health Organization 2019f) Indeed, 712 attacks against health care services and its personnel were reported in 2018, leading to 151 deaths. (World Health Organization 2018b) There has been a blurring of military-humanitarian-civil cooperation and

\footnotetext{
11 The Global Health Workforce Network was established in 2016, following a request by select Member States and building on a proposal by the Board of the Global Health Workforce Alliance. The Network operates within the WHO as a global mechanism for stakeholder consultation, dialogue and coordination on comprehensive and coherent health workforce policies. (WHO, 2016 https://www.who.int/hrh/network/en/)

12 The agenda, program and outcome statement of the $4^{\text {th }}$ Global Forum on Human Resources for Health, Dublin, Nov. 2017 can be found here: https://www.who.int/hrh/news/2017/action-to-avertanl8-million-health-worker-shortfall/en/
} 
roles while addressing epidemics such as Ebola and Zika during the last years. While this provides possibilities to enhance health security it could also impact the legitimacy of health care workers. Careful consideration and planning on the deployment of the military in health emergencies as well as that of health care workers in conflict situations are required. (Kamradt-Scott et al. 2016) Finally, there are international and regional human rights and social protection frameworks that can function as a normative basis for HRH development. This human-rights based approach has been somewhat overlooked in recent years and could still provide a strong drive for the need to develop a skilled workforce working towards health equity. (Campbell et al. 2013)

The outcome and functioning of these three political and governance spaces for HRH is determined by international policy structures and inherent tractions created in 'the political trilemma' surrounding global HWF development. Different policy imperatives and ideologies, whether based on economic investment, security, charitable, or cosmopolitan approaches will influence the policy space for HWF development. While there might be space within the trilemma to develop the workforce in a sustainable manner, there are fundamental questions on what would comprise a legitimate policy trajectory. One could imagine an open trans-national labor market where nation states actively promote HWF mobility and employability. However, this might not meet popular demand by citizens and health workers visà-vis labor rights and social protection. The 'Gilets Jaunes' movement is an example of the political expression that can arise from a part of the workforce becoming precarious. One could also imagine that countries want to 'protect' their health labor market-this is one of the drivers behind the Brexit demands in the UK. However, this might have an impact on the economy as a whole given how integrated our labor markets and interdependencies have become. Lastly, one could also imagine much more fiscal and political power at the supra-national level to regulate labor markets, taxation, social protection, and income policies across countries. There is a slow but increasing trend of increasing regional cooperation and standardization on health personnel accreditation, not only in the EU but also in regional economic integration organizations such as Association of Southeast Asian Nations (ASEAN) in Asia and Economic Community of West African States (ECOWAS) in West Africa. Nevertheless, in the current multi-polar world order nation states focus first on their own sovereignty including on how to organize their health system. Actors active in this complex HRH governance space need to be skillful and demonstrate leadership and diplomacy when navigating this policy landscape to further inclusive HWF development outcomes.

I aim to analyze the hypothesis that a compromise might still be possible in the globalization paradox, despite sides of the political trilemma providing intrinsic 
and strong tractions. Such a compromise would respect and advance a form of labor market integration, national sovereignty as well as democratic principles. My thinking is that a compromise follows, in essence, the cosmopolitan outlook as proposed by Beck. This would be unavoidable given the interconnectedness of our societies, health care labor markets as well as responding to the common risks, the public 'bads', that require shared solutions. These include the risk for pandemics, but also the health impacts of ecological degradation, climate change and growing income inequalities within and between societies. Governance arrangements across the three spaces, their inclusiveness, and legitimacy might advance HWF development, but could potentially also undermine it, depending on the tractions provided by the trilemma.

The global political economy distributes risks and opportunities in an unequal way. This applies to the workforce and likewise to the communities and patients that require these services. An inherent injustice exists in HWF development and the capabilities of people and societies to pursue essential health services. This thesis explores the three governance spaces and its inherent political trilemma. The several studies and analyses that constitute this manuscript aim to identify policy pathways and imagination on how to address the global HRH problematic in a sustainable and constructive manner. It applies a cosmopolitan outlook to advance understanding and possibilities to advance strong health systems, while imagining that everyone, everywhere could have access to a health worker. (Global Health Workforce Alliance 2011)

\subsection{OUTLINE}

The structure of this doctoral thesis follows the HWF governance and trilemma framework. Chapter 2 describes the methodologies applied in the studies and analyses that constitute the content of this manuscript. Given the different levels (see the Venn-diagram in Figure 1.3) and approaches required to capture a view of this complexity the study addresses several governance challenges, actors, processes, and methodologies. The methodologies include (comparative) policy analysis and case studies on policy implementations. It deconstructs and analyzes the application of governance frameworks as well as democratic, political and economic principles to global health institutions and policies crucial for advancing UHC and HWF development. Beck's arguments for the need to shift thinking, policy, and practice into a reflexive modernity and related cosmopolitan outlook policy arrangements will be a leitmotiv in the four chapters. The limitation of this doctoral thesis is the breadth of its scope. Each of the research areas (chapters) could empirically be elaborated into a separate thesis. However, this transversal, multi- 
level analysis has been deliberately chosen to advance insights and thinking into the HRH governance complex and its political drivers. This overall methodological approach to research several aspects of the governance complex as envisaged in Figure 1.3 reflects developments in the study of polycentric governance of complex economic systems. (Ostrom 2010) It also takes into consideration the emergence of transnational global governance networks that in a mixed form of hard and soft power aim to "collectively identify and solve problems on a global scale". (Slaughter 2003) This implies that this thesis is not an in-depth analysis of a specific problem following a linear causal relationship. Rather, it acknowledges that global HwF development is a networked complex adaptive system shaped by socio-political determinants. Each research area has been explored via case-studies and policy analyses that have been separately published as academic articles.

Chapter 3 covers the governance of the global health workforce via two studies. The first study traces the development of HRH policy and health systems reform following and after the Ebola outbreak in Guinea in 2014-2015. The outbreak raised a lot of international attention as well as fear. The study explores to what extent the Ebola outbreak provided the momentum and context for a sustained reform and expansion of HWF development in this West-African country. The second study is a policy analysis tracing the implementation of commitments made by governments and other actors at the $3^{\text {rd }}$ Global Forum on HRH. It compares and analyzes which policies and HRH actions have worked well for countries, what has been more difficult and what could be the shared approaches and mechanisms by relevant international actors to advance the HRH agenda. Chapter 4 covers the global health workforce governance by two studies looking into the wHO's reform process, the principles behind it as well as the implementation of the Global Code governed through the WHO. The first study concerns an analysis of the democratic legitimacy of the WHO and how principles of legitimacy (could) have been integrated into the WHO's reform and finance process to strengthen transnational Governance in Global Health. The second study looks into and compares the implementation of the WHO's Code of Practice in Europe and the Eastern and Southern Africa regions. It discusses the relevance and effectiveness of the code and how its governance and implementation can be strengthened in the nearby future. Chapter $\mathbf{5}$ covers global governance of the Health Workforce by one study and two analyses on how security practices, and the related securitization of health, have shaped thinking on health systems development and its priorities, including the role of health workers. I have chosen to apply the securitization of health as the main discourse (image) to analyze workforce trends in this chapter. A reason for this is that the economic discourse shaping the workforce agenda will also be covered in the chapter afterward. The main study in chapter 5 assesses why attacks on humanitarian health workers working in conflict areas have increased. Secondly, it wants to assess whether there is 
an erosion of humanitarian space and whether humanitarian health workers have been complicit in undermining this space, even if unwittingly. The study calls on humanitarian policymakers and health care practitioners to think about the political nature of their activities. Two short analytical perspectives follow this. The first perspective argues that the Ebola outbreak in West-Africa in 2014-2015 could have been prevented if health systems finance in the countries had not been constrained by fiscal limitations set by international institutions and if there had been proper investment in the HWF. The second perspective interrogates the 'resilience' concept and how it is applied to health systems development. The resilience frame captures democratic procedures and priority setting in public health agendas by 'claiming' an exceptional policy space, focused on risk containment and security objectives. Chapter 6 provides a cosmopolitan outlook on GHG and HWF development. It explores the political trilemma by exploring how different discourses shape and direct global health finance, governance, and HWF development. This chapter also consists of one study and two analytical perspectives. The study analyses whether GHG in the SDGs is grounded in the Right to Health. It assesses four core functions of the global health system from a normative perspective. Current representations of the right to health in the SDGs are insufficient and superficial because they do not explicitly link commitments or the right to health discourse (cosmopolitanism) to binding treaty obligations for duty-bearing nation states or entitlements by people. The first perspective provides the analysis that there is a fundamental contradiction in the SDGs between the objectives of 'sustainability' and 'economic growth'. While GHG in the SDGs deserves to be strengthened it is required to move beyond such a global policy frame by imagining sustainable and equitable health systems that are not based on capitalist notions of growth and development. The last perspective likewise questions economic growth as the main driver for HWF development. It argues for more inclusive and sustainable development models to accelerate workforce development both in LMICs as well as in HICs. Chapter 7 provides a discussion and reflection on the interrelation between GHG and global HWF development based on a cosmopolitan outlook as studied in the several chapters. It will provide a reflection on the framework and applied methodology. It will suggest policy pathways and the paradigmatic change required to accelerate global HWF development. 


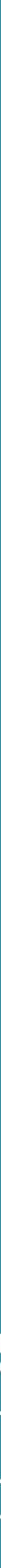




\subsection{METHODOLOGICAL APPROACHES STUDYING GLOBAL HEALTH}

The combined frameworks concerning Global Health Governance (GHG) for health workforce $(\mathrm{HWF})$ development and the embedded political trilemma require me first to reflect on how to approach methodologically the global HWF problematic.

Traditionally such a methodological approach follows an 'explanatory nationalism'. Koplan and colleagues wrote in 2009 that global health study and practice should move beyond a public and international health scope of analysis. (Koplan et al. 2009) International health's focus has been traditionally on health work 'abroad' (and public health arguably being 'here'). The focus in international health research is on developing countries, and with mainly a concern for infectious diseases, maternal and child health and malnutrition. The units of analyses (denominators) are national, district or local health systems. Through this analytical lens, countries can be compared with each other over time to identify positive or negative factors shaping health systems, mortality and other factors. Most famously, Hans Rosling takes such a comparative approach with his Gapminder Foundation ${ }^{1}$ and in his posthumous work 'Factfullness'. (Rosling 2019) In contrast, Koplan and colleagues argued "Not to restrict global health to health-related issues that literally cross international borders... global refers to any health issue that concerns many countries or is affected by transnational determinants... The global in global health refers to the scope of problems, not their location." (Koplan et al. 2009) This global health approach has been deepened via the concept of 'boundary-spanning' as a research and learning mindset that goes $a$. upstream: to draw out general or global lessons from the particulars of the local; $b$. downstream: for effectively applying global guidance for local practice and evaluating its relevance; and $c$. also laterally: for learning from different and comparable contexts. (Sheikh et al. 2016) Regardless of the concept scholars ask questions whether global health has really diverged from international health and its 'national fallacy'. (Beck 2007) Could it be that the distinction between international health and global health is in name only; differing brands but the same substance? Abimbola argues that global health should be decolonized and that such a 'glocal' approach could be best analyzed inductively by understanding information and motivation problems that limit and constrain delivery beginning at the local level. (Abimbola 2018)

Ruckert and colleagues make the case that as to improve the theorization on Global Health Diplomacy (GHD), it useful to determine what levels of analysis (international/

\footnotetext{
1 "Gapminder is a fact tank, not a think tank. Gapminder promotes a fact-based worldview everyone can understand. Gapminder shows the world history as if all countries of today had always existed and as if they always had the borders they have today. This is absolutely wrong, but it's necessary to make the animations easier to understand". (Gapminder, 2019 https://www. gapminder.org/data/geo/changes/)
} 
national/local) should be considered a priority under what specific circumstances and for what specific health issues. (Ruckert et al. 2016) As depicted in the Venn diagram in Figure 1.1 on GHG, it is important to analyze the global health issue at stake realizing that these three 'spheres' are permeable, have some transnational elements and are not 'closed entities' of analysis. By using these different levels of analysis, scholars will be better positioned to articulate the conditions that contribute to specific GHD outcomes. (Ruckert et al. 2016) Moreover, it is recommended that "The tools of political science could be harnessed by the field of global health to better understand global health's political dimensions and contested spaces in the international system, and to identify reasons why attempts to improve global health are often more complex and convoluted than conceived. Of special importance here is how power (at the individual, national/domestic, and international/global level of analysis) can shape GHD outcomes, and the theorization of impacts of power on GHD outcomes". (Ruckert et al. 2016) I have applied the theory of the globalization paradox and its trilemma, directly and indirectly, in the analyses covered in chapter 6 to research the political dimension of the global HWF agenda.

\subsection{DIFFERENTIATING METHODOLOGIES IN STUDYING GLOBALIZATION AND HEALTH}

Browne and Labonté argue that it is needed to take a differentiating approach to better understand what globalization is and how it affects our lives. (Brown and Labonté 2011) They identify three approaches to conceptualize and research globalization: globalist, skeptic and transformationalist. A globalist approach conceptualizes globalization as an economically driven process that is moving humanity closer to a more unified world, one in which the disparities between borders, markets, economies, and cultures are radically reduced in favor of a more common global condition. (Garrett W Brown and Labonté 2011) A skeptical approach challenges the positive elements involved with a globalist reading. Skeptics would argue that that the benefits of globalization are largely restricted to, or asymmetrically skewed, towards High-Income Countries (HIC) in Europe and North America. (Brown and Labonté 2011) A transformationalist analytical lens focuses less on locating specific 'drivers' explaining globalization. Rather, it attempts to locate the various ways in which globalization symbolizes an unprecedented period of global interconnectedness and social transformation. Globalization is not inherently good or bad but the result of incalculable economic, cultural, and political transformations that are restructuring the ways in which we live. It is appropriate to think of globalization as a pluralistic phenomenon with its idiosyncrasies and anomalistic permutations. (Brown and Labonté 2011) These three conceptualizations have a direct influence on methodological orientations and choices in assessing empirical properties. Brown and Labonté argue for a multidimensional approach to globalization. 
While economic dimensions should be incorporated, likewise key sociological and historical concepts must be considered. Moreover, besides macro-level quantitative research, it is important to supplement this with micro-level qualitative analysis to capture processes of 'glocalization'. They also suggest including a more inductive observational approach to analyze globalization processes in addition to the more deductive theoretical conceptualization. Lastly, globalization is seemingly good and bad at the same time. It is dialectical in the Habermasian sense in that it often presents a thesis and an anti-thesis at the same time. (Habermas 2018) This last approach links with the conceptualization and research questions of this manuscript. Global HWF development could be transformational with positive as well as negative elements at the same time. It is suggested that a more differentiated and contextual approach should supplement traditional methods by looking at the intersections and social transformations between the local and the global. (Brown and Labonté 2011) I have chosen to apply such a multidimensional model as well, in researching the global HWF challenge across several levels.

\subsection{METHODOLOGICAL COSMOPOLITANISM}

Beck takes such a transformational approach further by his thinking on a cosmopolitan outlook. "The goal is to explode and expand the provincial national horizon of sociology through a methodological cosmopolitanism." (Beck 2006a, p. 72) In essence, Beck argues that 'reality' has become cosmopolitan and that one needs to take a much more inductive everyday interpretation of social reality. He proposes that globalization research in the social sciences should contain the national project and the same time extend it. This can be done by recognizing the interconnectedness and interdependence of national spaces. Secondly, boundaries are becoming blurred. Transnational discourses, networks, and 'liquid' processes need to be analyzed as such. Lastly, 'cosmopolitanization' is not mono- but multi-perspectival. "More precisely, it can and must observe and investigate the boundary-transcending and boundaryeffacing multi-perspectivism of social and political agents through very different 'lenses'. (Beck 2006a, p. 82) A single transnational phenomenon, like HWF mobility, for example, can, perhaps even must, be analyzed "both locally and nationally and transnationally and translocally and globally". (Beck 2006a, p. 82) This realization is a major reason why I have chosen to approach the HWF problematic, including its transnational elements and governance, via three overlapping, blurring, multi-level spaces. Beck clarifies that this cosmopolitanization also brings with it a 'politics of perspectives' by creating new forms of conflicts within and beyond the state. The national frame is no longer valid as a shared arena for settling conflicts. At the same time, can transnational identities create a form of integration by facilitating and improving transnational and global cooperation and integration in times of global risks and 
crises? The political trilemma also reflects on this conflict and form of integration, It brings with it political drivers for conflicts as well as integration in advancing HWF development within and beyond the nation state. Finally, Beck argues that many issues that are celebrated as national are in essence increasingly transnational and cosmopolitan. 'The relation between science and the social word is thereby becoming paradoxical. Social structures and processes are becoming cosmopolitan, whereas scientific knowledge remains beholden to the axiomatics of the national'. The cosmopolitan outlook requires a dialogical imagination in everyday practice and in sciences. It forces us to develop the art of translation and bridge-building. (Beck 2006a, pp. 85-91)

\subsection{HEALTH WORKFORGE DEVELOPMENT AND ITS GOVERNANCE}

The Alliance for Health Policy and Systems Research (AHPSR) and the World Health Organization (WHO) published a Health Policy and Systems Research (HPSR) reader on HRH in 2017. (George et al. 2017) The reader presents the diversity of research methodologies and questions that are valuable to understanding HRH. The editors make the case that in HPSR, there is no hierarchy in evidence in contrast to what is used in the epidemiological sciences. The HSPR reader argues for a methodological fit dictated by the research question asked and its intended interference. This HRH research inference could take different forms and is classified as follows: descriptive, exploratory, explanatory, emancipatory, influence, predictive. (George et al. 2017, pp. 1315) Four studies in this thesis relate directly to HWF development. The two studies in the third chapter, on HRH policy development post-Ebola in Guinea and the policy tracing of HRH commitments made during the $3^{\text {rd }}$ global forum on $\mathrm{HRH}$, are descriptive studies. Descriptive in the sense that it outlines HRH phenomena and policy developments within its context, as well as comparing HRH policy actions between countries. Both studies consist of a mixed-methods approach whereby quantitative HRH data complement a policy analysis applying the health policy triangle tracing HRH policy development and implementation via qualitative data collection, including surveys and actor interviews. (Buse et al. 2012, pp. 8-18) A third study, in chapter four, explores the relevance and effectiveness of the wHO's Global Code reviewing its implementation in Europe and Eastern- and Southern Africa. It applies a case study approach to hypothesize why Code implementation is so different between the European and African regions. The study in chapter 5, on the securitization of health and its relation to the increased attacks on humanitarian workers explains why a securitization approach has an impact on the safety of health professionals. It provides a deconstructive and historical analysis on the relation between securitization, health as a bridge to peace, humanitarian space, and the role of health professionals in these spaces. 
For this thesis, I have not chosen to approach HWF development and its mobility from an HSPR perspective only. It applies a transformative approach to the study of globalization and health by assessing HRH complexities across the three overlapping political spaces of global health governance within and beyond the national outlook. The dialectic on globalization and health is pursued by not only taking as a basis an integrative form (globalism) of global HWF governance but also providing theory and empirical analysis on the more skeptical, political-economy, perspective of workforce requirements and its finance. This is represented in the 'political trilemma' analysis that functions as a form of anti-thesis. Moreover, a multi-disciplinary approach is chosen to capture the complexity of HRH governance. Besides the HSPR methodology that builds on health policy analysis and mixed-method approaches, the studies in the chapters apply political science concepts to explain the GHG and HRH phenomena. This includes an analysis of the input and output legitimacy of the (ongoing) reform of the WHO. It looks at the securitization of health as a broader political discourse shaping health and humanitarian policy. The development, or lack thereof, of health as a global public good and a human right is analyzed in tracing GHG in the development of the Sustainable Development Goals (SDGs). The international HWF agenda has is analyzed via a political-economy analysis and deconstructs the several governance discourses shaping workforce development as well as health systems conceptualized in the SDGs. I have applied a sociological lens by analyzing the concept of resilience in relation to health systems development from a Foucauldian governmentality perspective.

Moreover, in the discussion part of this thesis, I will explicitly return to the cosmopolitan outlook and reflect how the interconnectedness and interdependence of national spaces, as covered in several chapters, shape the outcome of HWF development, including possible pathways for the nearby future. More explicitly, I will reflect whether the cosmopolitan outlook is, or could be seen, as a compromise approach to overcome the gridlock in global HWF governance across its three political spaces and its political trilemma. A multi-perspective analysis reflecting on the development of contemporary HWF governance complexity will be applied in the discussion too. This follows theoretical work on transnational networked governance models by Slaughter who argues that "Global justice is a noble but sadly distant ideal. Global disorder is more evident than order. But in the everyday rhythms of regulators around the world, new forms of global governance are being born". (Slaughter 2003) Moreover, it will discuss whether a polycentric analysis of complex governance systems in the economic realms (see e.g. Ostrom 2010) following our main framework (Figure 1.3) is actually appropriate to advance scientific insights, policy, and practice concerning HWF development. 
The next section will describe shortly the methodologies applied in each chapter and per study.

\subsection{PLURIFORM METHODOLOGIES}

Chapter 3 Includes two studies. The first study aims to assess the dynamics of HWF retention in rural areas in the post-Ebola period in Guinea and assesses the availability of HWF education in relation to the labor market supply. The conceptual framework and structure for this study follow Gilson and Walt's health policy triangle that indicates how different actors (individuals, government, and national/ international organizations) interact to influence planning, implementation, and evaluation of health policies. (Buse et al. 2012, pp. 8-18) This study is a mixedmethods approach with data collected at national, regional, and district levels between October 2016 and March 2017. Interviews were realized with 57 key actors involved at multiple levels of capacity development, training, and management of the HWF, both in and outside government. Qualitative data were collected through semi-structured interviews based on pre-tested interview guides. This included questions on how education profiles match with labor market needs, HWF retention dynamics in the rural districts and recruitment policies. Directors of the seven health professionals' schools in each administrative region of Guinea were interviewed to obtain data on the health workers (HWs) trained during the preceding 5 years and to assess whether these nursing/midwifery schools were functioning in the post-Ebola period.

The second study traces the implementation of HRH policy commitments made by 57 countries and 27 other entities at the third global forum on HRH in Brazil in 2013. The objective of this tracing study is to analyze the implementation of the commitments by holding policymakers to account and by generating insights and evidence on the relevance, effectiveness, and results of the HRH policy actions. Between February and June 2016, the 57 governments and 27 other entities were approached. The baseline data for the policy tracing consisted of the HRH commitments and related analysis conducted by the WHO in 2014. A desk-based analysis consisting of a scoping review of the existing literature on HRH activities in each country was conducted. In the second part of the policy tracing study, the assessment of the outcome of the HRH actions was complemented by applying the health policy triangle. (K Buse et al. 2012) An online survey and a guideline for semi-structured interviews were developed to collect data to be provided by the representatives from the governments and other entities. Triangulation of research data was performed by cross-checking available literature, policy documents and grey literature, and through verification of the collected data by the wHO country staff. 
Chapter 4 covers two studies. The first looks into the wHO's reform process and its governance principles. The second traces the implementation of the Global Code. The study on the democratization of the wHO explores if and whether it can really be the key health authority in a globalized world based on a cosmopolitan democracy. Democratic legitimacy in transnational governance arrangements such as at the wHO can be conceived as a five-faced prism whose surfaces are respectively: (1) representation; (2) accountability; (3) transparency; (4) effectiveness; and (5) deliberation. (van Ham 2013) The study used this legitimacy framework to deconstruct and explore the different elements of input and output legitimacy in the wHO's reform process. It used both academic as well as grey literature to provide this analysis. In addition, the study describes the wHO's governance and finance arrangements with external partners. The study on the implementation of the wHO's Global code has as an aim to assess its relevance and effectiveness. The study analyses and compares its implementation in two regions of the world via the availability of several case studies conducted by the authors. In the European region, case studies from the European Union's (EU's) Development Cooperation Program "Health Workers for all and all for Health Workers" are included in the analysis. (Healthworkers4All 2015) In the African region, this included analysis on the Code implementation in 10 countries by EQUINET (European Network of Equality Bodies), the Regional Network on Equity in Health in East and Southern Africa. (Dambisya et al. 2013) The data generated by these two programs informed the overall analysis of this paper.

Chapter 5 includes a study that explains why attacks on health care workers working in conflict areas have increased. Secondly, it tries to assess whether there is an erosion of humanitarian space and whether humanitarian health workers have been complicit in undermining it. It covers a (historical) explanation and scoping review of the main theoretical concepts in relation to violence directed towards humanitarian workers. This includes issues such as 'The Securitization of Health' (Rushton 2011), 'Humanitarian Space' (Collinson and Elhawary 2012), 'Health as a Bridge to Peace' (Rushton and McInnes 2006), and a classification of health workers' role in violent conflicts and humanitarian settings. (Buhmann et al. 2010) The paper explores the modern role of non-governmental organizations (NGOs) and development cooperation for health under the banner of liberal humanitarianism and GHD. Three empirical cases covered in the literature support the argument. The discussion brings these components together and nuances them by providing a historical comparison to practices during the Second World War. Two shorter analytical pieces complement the chapter. The first one concerns the Ebola epidemic in West-Africa from 2014-2015 and makes a political-economy analysis that the epidemic could have been prevented if the countries would have had the fiscal space to invest in their health system. (Kentikelenis 2017) It explains the concept of PostWestphalian (cosmopolitan) public health (Fidler 2003) and why this approach is 
necessary to secure GPG's such as health security. The second piece is a critique of the resilience discourse and how it is applied to health systems policy development. The resilience concept is deconstructed as a mode of self-governance that, in the sense of bio-politics and governmentality, is an ideal, modern and appropriate method to manage populations. (Foucault 2009) A case study from Gaza supports the argument and clarifies why and how vulnerability and resilience aspects are intimately connected in relation to its health system functioning.

Chapter 6 provides then a cosmopolitan reflection and outlook on GHG, HWF development and its political trilemma. The main study in this chapter explores the extent to which GHG-in the context of the early implementation of the SDGS-is grounded in the Right to Health. First, the Human Right to Health is defined in the context of the SDGs. (Ooms et al. 2014) Secondly, the four functions of the global health systems as defined by Frenk and Moon are analyzed from a normative right to health analysis. These functions include stewardship, the production of global public goods, the mobilization of global solidarity, the management of externalities. (Frenk and Moon 2013) Two analytical papers complement this study. The first paper explores whether global health progress and attainment of the SDGs is possible under current models of global governance based on capitalist values. Two different paradigms explore the advancement of global health objectives in the SDGs. The first paradigm is, in essence, "a gigantic global version of Franklin Roosevelt's New Deal". (Labonté 2016) This is a Neo-Keynesian investment model in green growth, decent employment, social protection, and provision of public services. The second paradigm touches upon the necessity to respect planetary boundaries and address anthropogenic depravation. Ecological-centered values must be central in any future global governance of health framework. The latter forces researchers, policymakers and professionals to apply insights from alternative governance models such as on circular economies (Raworth 2017a), post-capitalism (Mason 2016) or even Degrowth policies. (Hickel 2019) While GHG is in general comfortable to function within this first paradigm, it has so far only very limitedly engaged with the need to shift to this second paradigm (a 'reflexive' modernity). The last paper takes this argument further by analyzing GHG models relevant to the global HWF agenda from a political-economy perspective. It makes the argument that HWF development must incorporate models and thinking beyond economic growth. It analyses the (im)possibility to expand fiscal space for HWF investments in Low- and Middle-Income Countries (LMICs) as suggested by the wHO. (Buchan et al. 2017, p. 236) Consecutively a political-economy analysis is conducted to interrogate the fiscal space available in LMICs to invest in the workforce. (Milanovic 2016a; Rodrik 2011a) The article proposes then to use a more differentiated approach to global HWF development by applying different "policy frames', a cosmopolitan outlook, that could be pursued by states and others while cooperating on global health issues. (Labonté and Gagnon 2010) 


\subsection{METHODOLOGICAL LIMITATIONS AND CHALLENGES}

The main limitation of this thesis is the breadth of its scope by analyzing the elements of, and the interrelation between, the spaces and politics relevant for global HWF development. This has been done deliberately to capture complexity and multiple perspectives. This also implies that the studies, especially those in chapters 3,4 and 5, capture only a minor part of the multiform HWF 'image' covered by the HPSR reader on HRH. (George et al. 2017, p. 23) This thesis covers mainly the outer layer of this HRH problematic, especially regarding the economic context; politics; conflict setting and its governance modalities. Only the first study on HWF development in Guinea post-Ebola addresses local issues such as retention, training, motivation, and interactions at the micro-level. The study in Guinea provides an inductive understanding of the (im)possibilities of HWF to be retained in rural areas including how this is shaped by (global) social and economic forces. The other papers address governance, policy and institutional elements of HWF agenda at the (inter)national and transnational levels. This implies that many relevant HWF issues that play at the mezzo and micro-level such as improving gender equity, building HWF capacity, enhancing $\mathrm{HRH}$ performance, intrinsic motivation, leadership, management, etc. are not or only limitedly covered in this thesis. I have chosen this approach on purpose, because: A. Global governance studies have so far had minimal coverage in academic literature on HWF development whilst there is a considerable, worldwide, body of literature on HWF mechanisms, practices, and governance arrangements at the local level (George et al. 2017); B. For my public health master's thesis, I have already conducted a narrative research on local HWF policy practices in an LMIC and conflict-setting, more specifically in the province of West-Papua, Indonesia. (Remco van de Pas 2010) Practicing medicine and conducting research in this context made me realize that it is important to include upstream and political determinants of health. (Rees et al. 2008) It has led me to focus more on national and multilateral policy processes influencing the HWF. This doctoral thesis builds forward on the research experience in West-Papua.

The studies covered in this thesis have been funded by, and are part of several research-and-development cooperation programs. The studies hence were shaped by the objectives of these programs. Moreover, over the course of this thesis project I have occupied different roles at three different organizations; two of them academic institutes, and one an international public health NGO. These programs and roles have considerably influenced methodologies and approaches taken in the study. For instance, the study in chapter 3 on tracing HRH policy commitments by 57 countries was commissioned by the WHO. Given its mandate (governed by sovereign nation states), it is logical that the unit of analysis and comparison are countries, and not transnational or global processes. The studies in chapter 4 were conducted while 
I was working for a public health NGO. The commissioned papers explicitly asked for an NGO perspective on the wHO governance as well as the Global Code, which allowed for the application of a legitimacy framework as well as a comparison on case studies collected in a Europe-wide project. Chapter 5, on humanitarian aid and its securitization, has been influenced by my personal experience working as a humanitarian health practitioner (2005-2008) and active role in the governance structures of a humanitarian NGO network (2010-2014). This has likely shaped my perspectives and is potentially a form of bias. Lastly, the study on the Right to Health and GHG was part of a research program funded by the EU that aimed at providing policy recommendations for the EU's position in what have eventually become the SDGs. From the beginning of this project, there were normative (human rights) considerations in moving Universal Health Coverage (UHC) forward as a priority SDG goal for the EU. This aim provided a specific perspective on how to advance GHG and was hence an inherent 'cosmopolitan' outlook propagated by the research group. I will reflect in the discussion how my positionality as a researcher and the funding and institutional arrangements behind the studies have influenced the eventual outcome and analysis in moving the Global HwF Agenda forward. 


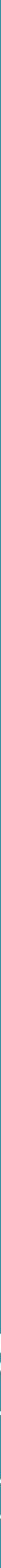




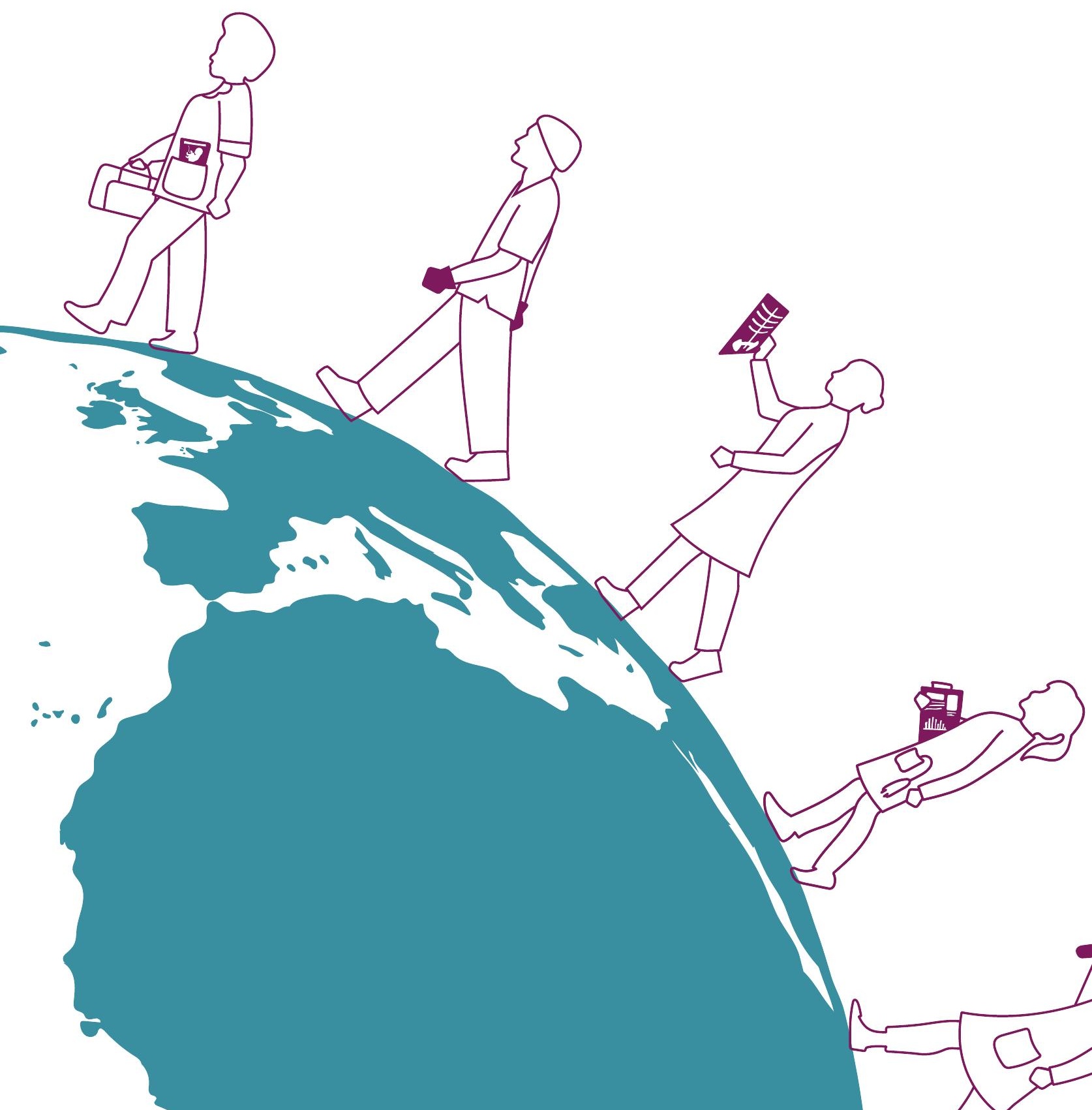




\title{
3.1
}

\section{Health workforce development and retention in Guinea: a policy analysis post-Ebola}

\author{
van de Pas, R., Kolie, D., Delamou, A., \& Van Damme, W. (2019).
}

Health workforce development and retention in Guinea: a policy analysis post-Ebola. Human resources for health, 17(1), 1-12.

https://doi.org/10.1186/s12960-019-0400-6 


\begin{abstract}
Background: The state of the Guinean health workforce is one of the country's bottlenecks in advancing health outcomes. The impact of the 2014-2015 Ebola Viral Disease outbreak and resulting international attention has provided a policy window to invest in the workforce and reform the health system. This research constitutes a baseline study on the health workforce situation, professional education, and retention policies in Guinea. The study was conducted to inform capacity development as part of a scientific collaboration between Belgian and Guinean health institutes aiming to strengthen public health systems and health workforce development. It provides initial recommendations to the Guinean government and key actors.
\end{abstract}

Methodology: The conceptual framework for this study is inspired by Gilson and Walt's health policy triangle. The research consists of a mixed-methods approach with documents and data collected at national, regional, and district levels between October 2016 and March 2017. Interviews were realized with 57 resource persons from the Ministry of Health, other ministries, District health authorities, health centers and hospitals, health training institutions, health workers, community leaders, NGO representatives, and development partners. The districts of Forecariah and Yomou were chosen as the main study sites.

Results: Limited recruitment and a relative overproduction of medical doctors and nurse-assistants have led to unemployment of health personnel. There was a mismatch between the number of civil servants administratively deployed and those actually present at their health posts. Participants argued for decentralization of health workforce management and financing. Collaboration between government actors and development partners is required to anticipate problems with the policy implementation of new health workers deployment in rural areas. Further privatization of health education has to meet health needs and labor-market dynamics. 


\section{BACKGROUND}

The state of the Guinean health workforce (HWF) is one of the country's bottlenecks in advancing health outcomes. (Ministère de la Sante de Guinée 2016) There has been a decade-long underinvestment with limited public recruitment and a workforce dominated by medical doctors. (Alexander Kentikelenis et al. 2015; Ministère de la Sante de Guinée 2017) In 2014, a workforce projection study found that maternal and neonatal health services require particular attention. The main shortage is in skilled birth attendance where only $18 \%$ of needs are met. (Jansen et al. 2014) Although there is a relative oversupply of general practitioners and nurse-assistants (ATS), many of them work in the private, informal sector. There are considerable variations in the distribution of health personnel given the Human Resources for Health (HRH) needs and HRH supplies between rural and urban areas. (Jansen et al. 2014) While overall HRH needs for maternal and neonatal health services were projected to increase by $22 \%$ between 2014 and 2024, the supply was projected to decline by $15 \%$ under existing recruitment patterns. (Jansen et al. 2014)

The Ebola Virus Disease (EVD) outbreak of 2014-2015 facilitated international finance and humanitarian assistance and spurred the United Nations (UN) Security Council to create the first ever UN mission for a public health emergency. (United Nations Security Council 2014) In the wake of EVD, there has been much debate and proposals for global health governance reforms by the World Health Organization (WHO) and other institutions to address future epidemics and build resilient health systems. Authors have suggested that the EVD outbreak could be a transformative moment in recognizing that there are shared responsibilities by governments in strengthening health systems. (Kickbusch and Reddy 2015) The workforce is now considered a crucial pillar for global health security and has been included in the Sustainable Development Goals (SDGs) ${ }^{1}$. (The Lancet - Editorial 2016; UN Sustainable Development Knowledge Platform 2015) The WHO's Global Strategy on HRH: Workforce 2030 includes a new estimate of the workforce density required to meet the SDGs${ }^{2}$. (World Health Organization 2016a) This figure is ten times the proportion of HWF currently employed by the Guinean public sector. (Ministère de la Sante de Guinée 2016) A UN commission has also provided a report and action plan on the importance of health employment for economic growth. (World Health Organization 2016d) In Guinea itself, the impact of the EVD outbreak and resulting international attention has provided a policy window to invest in the workforce and

\footnotetext{
1 SDG Target 3c "Substantially increase health financing and the recruitment, development, training and retention of the health workforce in developing countries, especially in least developed countries and small-island developing States".

2 This target is set at 4.45 health workers/1000 population and is almost twice the first estimation made by the WHO in 2006 (2.28).
} 
reform the health system after many years of stagnation (See Figure 3.1). Indeed, the government implemented a health system recovery and resilience strategy with the intention to recruit 6,000 staff from 2016 to 2018 and increase their salaries by $40 \%$. (Ministère de la Santé de Guinée 2015b) In 2016, the Ministry of public services recruited 3,802 health workers (HWs) who signed a 5-year contract committing to work in rural areas and were deployed in March 2017. (Ministère de la Fonction Publique 2016) International development partners have provided much support to further strengthen Guinea's health system, including 25 million Euros from the European Union in 2015, provided that the government expands its fiscal expenditure on health. (ECORYS Consortium de Santé 2016) By 2017, the government increased the total health expenditure from $4 \%$ to $8 \%$. (Diallo 2017)

Meanwhile, the government has planned to reform HRH policy and management functions (Box 3.1).

Box 3.1: Reforms by the Guinean government in $\mathrm{HW}$ policy and management functions (Ministère de la Sante de Guinée 2016)

- To develop a national strategy to retain staff in remote areas via decentralized training and recruitment.

- Strengthen the institutional framework for HRH recruitment and management performance.

- Re-concentrate initial formation of health workers to improve the quality of care;

- Strengthen the capacity of health education institutions through intersectoral collaboration.

- Strengthen the skills of personnel in terms of quality of care and health management.

- Establish a strategy to develop and motivate a Community Health Workforce. (Diallo 2017)

- Reform of HRH policy and management is part of institutional developments in Guinea in which policy dialogue and health coordination structures have been established over the last years. (Ade et al. 2016)

Complementarily, the government has strengthened essential public health functions such as epidemiological surveillance by creating regional alert and response teams as well as a National Health and Safety Authority (ANSS). (Ade et al. 
2016; Agence Nationale de Sécurité Sanitaire de Guinée) Guinea's Ministry of Health $(\mathrm{MoH})$ has announced plans to invest in 11,000 new HWF jobs over the next decade. (Ministère de la Santé de Guinée 2015b)

This research constitutes a baseline study to inform scientific and educational capacity development as part of a scientific collaboration between Institute of Tropical Medicine (ITM), Antwerp and Centre National de Formation et de Recherche en Santé Rurale (CNFRSR), Maferinyah, aiming to strengthen public health systems and HWF development in Guinea. The study advances academic debates on how to further HWF development for resilient health systems in fragile contexts. (Witter and Hunter 2017) The objectives and analysis are informed by a framework for HWF labor market dynamics. (McPake et al. 2013) It provides initial recommendations to the Guinean government and key actors in improving education, retention, and sustainability of staff recruited to work in rural areas. Consecutive studies will analyze the policy process, implementation, and health systems impact of employing HWF in remote areas. Two main research objectives have been identified:

1. To assess the dynamics of HWF retention in rural areas in the post-Ebola period.

2. To assess the availability of HWF education in relation to the labor market supply.

This study was conducted after the policy decision to employ HWs in rural areas, but before the actual recruitment and deployment in 2017. The study intends to provide policy guidance facilitating the actual retention of HWF. This article is a short version of a research report presented to the $\mathrm{MoH}$ and other relevant actors in a workshop in September 2017. (Kolie et al. 2017)

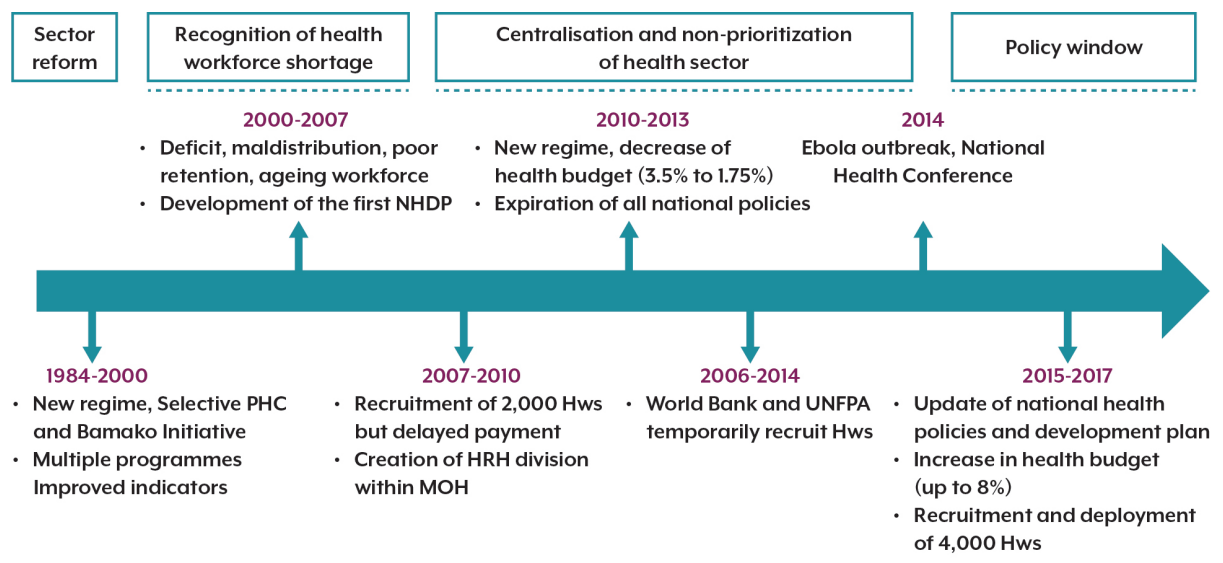

Figure 3.1: Evolution of HRH policy in Guinea Post-Ebola 


\section{METHODOLOGY}

The conceptual framework for this study follows Gilson and Walt's health policy triangle which indicates how different actors (individuals, government, and national/ international organizations) interact to influence planning, implementation, and evaluation of health policies. This model also helps assess perceptions, processes, and complexities of established strategies. (K Buse et al. 2012) In this study, the health policy triangle has been applied prospectively to anticipate the formulation and implementation of the new deployment policy. (K Buse et al. 2012) The different elements of the health policy triangle provide the structure for the results and discussion sections.

This study is a mixed-methods approach with data collected at national, regional, and district levels between October 2016 and March 2017. Interviews were realized with 57 key actors involved at multiple levels of capacity development, training, and management of the HWF, both in and outside government.

Table 3.1 depicts an overview of the study participants: respondents were purposefully selected and via snowball sampling, additional key actors were included.

Table 3.1: Number of interviews conducted per groups of participants

$\begin{array}{ll}\text { Groups of participants } & \text { Numbers interviewed } \\ \begin{array}{l}\text { Developments partners/donors, international local NGO representatives } \\ \begin{array}{l}\text { Officials of the Ministry of Health (central, regional and district } \\ \text { authorities) }\end{array}\end{array} & 13 \\ \begin{array}{l}\text { Officials of the Ministry of Education and Training Institutions } \\ \text { Officials of the Ministry of Public/Civil Services }\end{array} & 08 \\ \begin{array}{l}\text { Officials of the Ministry of Decentralization (regional and local } \\ \text { authorities) }\end{array} & 05 \\ \begin{array}{l}\text { Health workforce (civil servants working as health-facility managers or } \\ \text { caregivers, contractors, and volunteers) }\end{array} & 17 \\ \begin{array}{l}\text { Total } \\ \end{array} & \mathbf{5 7}\end{array}$

The health districts of Forecariah and Yomou were chosen as the main study sites, and represent two very different rural contexts. This first study focused on rural areas only given the gap between HWF needs and supplies between Conakry and the rest of the country. (Jansen et al. 2014) Given feasibility and timeframe, only two study-sites were selected at this stage. 
Forecariah is well accessible by road in two hours from Conakry and provides for personal and market mobility. Yomou is situated over a thousand kilometers from Conakry in the Guinean forested region close to the Liberian border. There are fewer government investments in infrastructure, education, and services than central Guinea. The HWF situation in Forecariah is less deficient than in Yomou, but the former has been severely affected by EVD, with 433 cases identified and $10 \mathrm{HWS}$ infected. In Yomou only 10 cases were identified.

Directors of the seven health professionals' schools in each administrative region of Guinea were interviewed to obtain data on the Hws trained during the preceding 5 years and to assess whether these nursing/midwifery schools were functioning in the post-Ebola period. A quantitative analysis was conducted to determine the professional graduate trends by year and type of personnel, which provide insights on the decentralized supply of HWs in the regions.

Qualitative data were collected through semi-structured interviews based on pre-tested interview guides. This included questions on how education profiles match with labor market needs, HWF retention dynamics in the rural districts and recruitment policies. The interviews were then fully transcribed. The two main researchers independently coded and analyzed the results following a coding grid that corresponded to different elements of the health policy triangle. Quantitative data were collected using a pre-developed and pre-tested form. At district levels, HWF registers were consulted and retention rates were calculated using the civil servants' registry. All participants received an information sheet and provided a signed consent to be included in the study. Study participants and key actors could provide feedback on the draft report during a research workshop in July 2017. The study was approved by the national research ethics committee in Guinea ${ }^{3}$.

\section{RESULTS}

The results are structured according to the two main research objectives. The first objective on HWF retention is presented along the 4 interrelated components of the health policy triangle: situational context, policy content, process, and actors involved. The quantitative results on HWF education are presented afterward.

3 Comité national d'éthique pour la recherche en santé (CNERS). No. 130/CNERS/16. Guinée, Conakry, October 11, 2016. 


\section{The dynamics of HWF retention}

\section{HWF Situation and contextual factors}

An absolute shortage of HWF in both districts according to global HRH policy guidelines ${ }^{4}$ was observed. This shortage was more or less similar between Yomou and Forecariah. In total, 289 Hws were registered in Forecariah and 135 in Yomou. This corresponds with a ratio of 1.2 available Hws per 1,000 persons in each of the districts (Table 3.2).

We also found an important mismatch (absenteeism) between the number of HWs deployed (according to the civil servant registry) and those actually working in the district (according to the district authorities). In fact, of 202 civil servants posted in Forecariah, only 83 were present at their post (retention rate: $41 \%$ ). This retention rate was 39\% (43 present of 111 posted) in Yomou. Some participants argued that most of the healthcare tasks were actually provided by local contractors or volunteers, who hope to be prioritized during civil servant recruitment processes.

Participants in the districts mentioned that the top-down model of civil servant recruitment does not favor retention in rural areas. Participants reported that the staff, purposefully recruited from the capital, was parachuted in underserved areas for just a few months to benefit their civil servant salary, while volunteers or contractors working for decades in the municipalities had fewer chances of being recruited.

"We have recommended the central level to recruit people already working with us as volunteers or contractors for years but they prefer recruiting those who are prepared for anything except staying in rural areas" (IDI 15, member district health office).

Furthermore, the available civil servants in both districts were unequally distributed as depicted in Table 3.2. Most of them worked in the urban conglomerations either at district management levels, district hospitals or urban health centers. In remote areas, contractors and volunteers were the most represented. Additionally, there was also an imbalance in the distribution of Hws according to their professional categories. Doctors, pharmacists, and dentists (type A), and nurses, laboratory technicians, and midwives (type B) were mainly found in urban areas while assistant nurses (type C) and informal community health workers occupied facilities in rural areas.

4 In 2016, "An SDG index threshold" of 4.45 doctors, nurses and midwives per 1,000 population was identified by WHO as an indicative minimum density representing the need for health workers. 
Table 3.2: Health workforce profiles and distribution in Forecariah and Yomou districts

$\begin{array}{lll} & \text { Forecariah } & \text { Yomou } \\ \text { Health workforce per status } & \text { Number }(\%) & \text { Number }(\%) \\ \text { Civil servants* } & 83(29) & 43(32) \\ \text { Contractual/volunteers } & 206(71) & 92(68) \\ \text { Total } & 289(100) & 135(100)\end{array}$

Health workforce distribution per status and categories in rural areas

Civil servants

$\begin{array}{lll}\text { Type A } & 5(6) & 0(0) \\ \text { Type B } & 4(5) & 7(16) \\ \text { Type C } & 14(17) & 9(20) \\ \text { Informal } & 0(0) & 0(0) \\ \text { Sub-total } & 23(27) & 16(37) \\ \text { Contractual/Volunteers } & & \\ \text { Type A } & 0(0) & 1(1) \\ \text { Type B } & 22(11) & 12(13) \\ \text { Type C } & 31(15) & 25(27) \\ \text { Informal } & 99(48) & 36(39) \\ \text { Sub-total } & 152(74) & 74(80)\end{array}$

Health workforce distribution per status and categories in urban areas

Civil servants

Type A

$12(14) \quad 5(12)$

Type B

$19(23)$

$11(26)$

Type C

$30(36)$

$11(26)$

Informal

$0(0)$

0 (0)

Sub-total

61 (73)

27 (63)

Contractual/Volunteers

Type A

$7(3) \quad 0(0)$

Type B

17 (8)

0 (0)

Type C

18 (9)

18 (20)

Informal

12 (6)

0 (0)

Sub-total

54 (26)

$18(20)$

*83 health workers were present out of the 202 posted in

Forecariah. In Yomou, this was 43 out of 111 health workers,

Guinea, December 2016 
HWF aging is another highlighted challenge; many HWs will retire in the coming decade. (Jansen et al. 2014) The main point emerging at local levels is that the state should accord sufficient resources and engage with development partners to favor decentralization of its recruitment, deployment, and payment policies. At the central level, participants identified three major issues: First, the state is solely responsible for its employees and should not have support from partners regarding their salaries; Second, several donors are, in principle, committed to supporting the state but this assistance has been arriving slowly;

"The EU wants to support the government in this process but the funding has not yet been received. The EU requires an indication of further deconcentration and decentralization of personnel management within the country." (IDI 21, development partner)

Third, partners committed to supporting the government in sustainable health system reforms have themselves recruited HWs into projects that they support.

\section{HRH policy on recruitment and deployment}

Participants reported that in the post-EVD period, staffing shortfall was temporarily solved by ANSS and the United Nations Population Fund (UNFPA) maternal health project contracting and deploying HWs. District health managers were worried about the contracts of ANSS HWs ending soon. At the district level, respondents suggested that contracted HWs and interns from the health centers are prioritized in the new recruitment; however, this should also be followed by an administrative decentralization of HWF management. HWF supervision was considered inefficient with limited decision space at district levels. Respondents also argued for annual recruitments rather than the currently-practiced five-yearly recruitments.

It was also suggested that the state take gender issues into account when deploying staff since married women may prefer to work in urban areas or near their husbands. Respondents mentioned that recruitment from outside the region had negative impacts mainly concerning responsibilities and trust between staff members.

Participants provided policy propositions for improved HWF retention. This includes local recruitment, strengthening supervision, allocation of wages by the local administration, including community overview, adopting career plans and rotation schemes for staff, developing medical specialist positions in rural hospitals, improving living and working conditions, and creating incentives (financial) and a particular status for health staff working in rural areas. One participant confirmed that there are ongoing reflections to initiate real reform of the national human resources policy. 
"The state needs to consider creating a local public service in every region of the country. This service could be directly under the authority of the regional governor and would be responsible for recruiting state officials, including health professionals." (IDI 14, government officer)

\section{HWF policy development}

Some local actors reported that central public administration officials would be the first to undermine a new transparent retention policy. They would do so by favoring persons during the deployment process or by refusing to adopt punitive measures against those who would not stay in their job positions. Many Hws recruited during the last round did not comply with their contract of engagement.

Approximately $40 \%$ of participants were in favor of a five-year service contract working in rural areas and some were ready to be deployed to such areas. Respondents believe that new staff deployment will have a positive impact on improving coverage and provision of care. Nevertheless, others said that the lack of state transparency in HWF management is a demotivating factor in practicing health services in rural areas.

"In other countries, people line up to work in rural areas as it allows them to access training grants but at ours, those who refuse to go to the interior are the same ones who could finalize their studies abroad or who are promoted to positions of responsibility?" (IDI 30, health worker)

Although all respondents appreciated the new recruitment of HWs, many had a negative perception of its organization and reported low recruitment rates of specialists. Respondents argued that the state should pay more attention to collaboration with the private sector which could be a real driver of employment and growth for the country.

\section{Actors, values, positions, and collaborations on HWF policy}

Educational and ministerial actors who are responsible for the training, recruitment, and central management of HWF confirmed that the MoH was not playing its full role in HWF management. According to them, there is an overproduction of less qualified HWs in the country. Additionally, their training curriculum does not follow the needs and priorities set by the $\mathrm{MoH}$. Respondents reported that the $\mathrm{MoH}$, which deploys HWs, collaborates poorly with the Ministry of Finance (MoF) regarding their payment.

"We do not control the training of health workforce, let alone its recruitment or salary. All these related services report to their line ministries and not to the Ministry of Health." (IDI 23, government officer) 
Central actors and respondents from a non-governmental organization (NGO) and a multilateral organization were of the opinion that the MoH's main task is the supervision of the HWF. They argued that poor coordination between ministries negatively impacted the sustainability of health gains. Moreover, MoH respondents had doubts about the functioning of an inter-ministerial HWF committee as a consultative platform. The ministry has proposed to transform the human resources division and create an HWF directorate but respondents argued that such institutional development should be accompanied by the allocation of more financial resources to strengthen capacities and staff supervision.

Representatives of international organizations reported that their institutions would recruit Hws in their areas of intervention and thereby accompany the state in implementing the health system's recovery and resilience plan. The development partners would be distributed between several regions to allow coverage of the whole country but also to evaluate the impact on the improvement of the system in each actor's area of intervention.

"We are recruiting health workforce with the highest criteria to run our health projects. The contract we have with this health workforce already perceived by the state as a hiring contract in the public service. The state does not respect its commitments and this poses a problem for maintaining this staff in the project areas." (IDI 24, development partner)

There has been progress in improving community-based surveillance through support from the International Organization for Migration. Additionally, both Forecariah and Yomou districts benefited from staff being recruited and contracted by the ANss. The district hospital management and health authorities reported good collaboration between them and ANSS staff. Although these managers appreciated the availability and motivation of ANSS staff, they entrusted also that this staff lacked experience in monitoring activities. ANss employees, meanwhile, reported difficulties in integrating immunization campaign supervision activities at the district level. They also struggled in participating in regular training activities organized by the district health team. This integration challenge is partly due to parallel financial and management systems.

\section{HWF education and its relation to the labor market supply}

The results in this section provide a quantitative overview of the HWF education in Guinea. Figure 3.2 indicates a mal-distribution of health training institutions across the country. Most are located in Lower Guinea, particularly in Conakry. Much of Middle and Upper Guinea lacks health training institutions. This disparity mainly concerns universities and professional schools (type B) which train state nurses, midwives, laboratory and public health technicians, and social assistants. 
Type A vocational schools that train ATs are more or less well-distributed throughout the country, although some type B vocational schools also train ATs. There are four higher education institutions (universities) in Guinea: three in Conakry (two are private) and one in N'zérékoré.

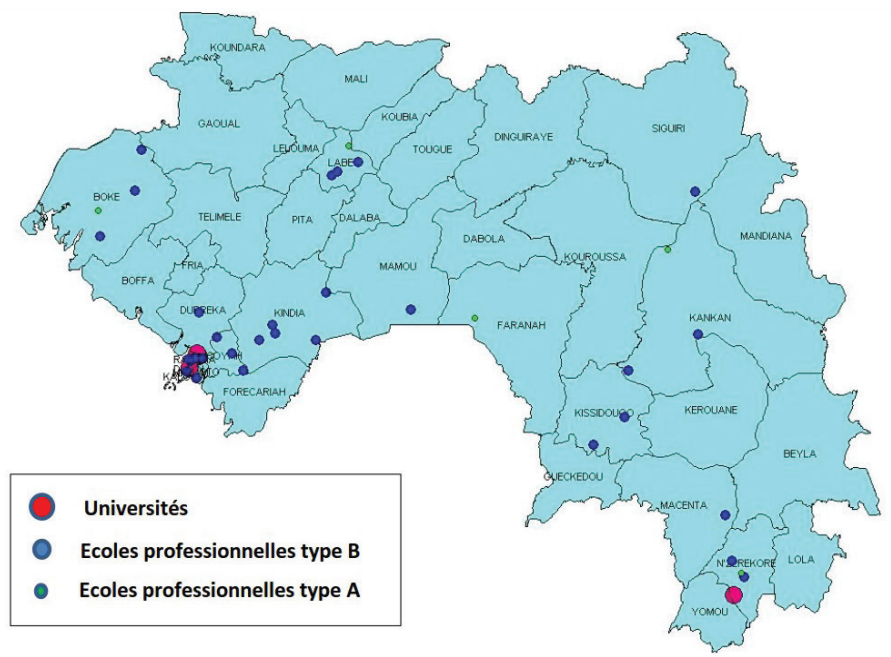

Figure 3.2: Geographic distribution of health training institutions in Guinea, April 2017
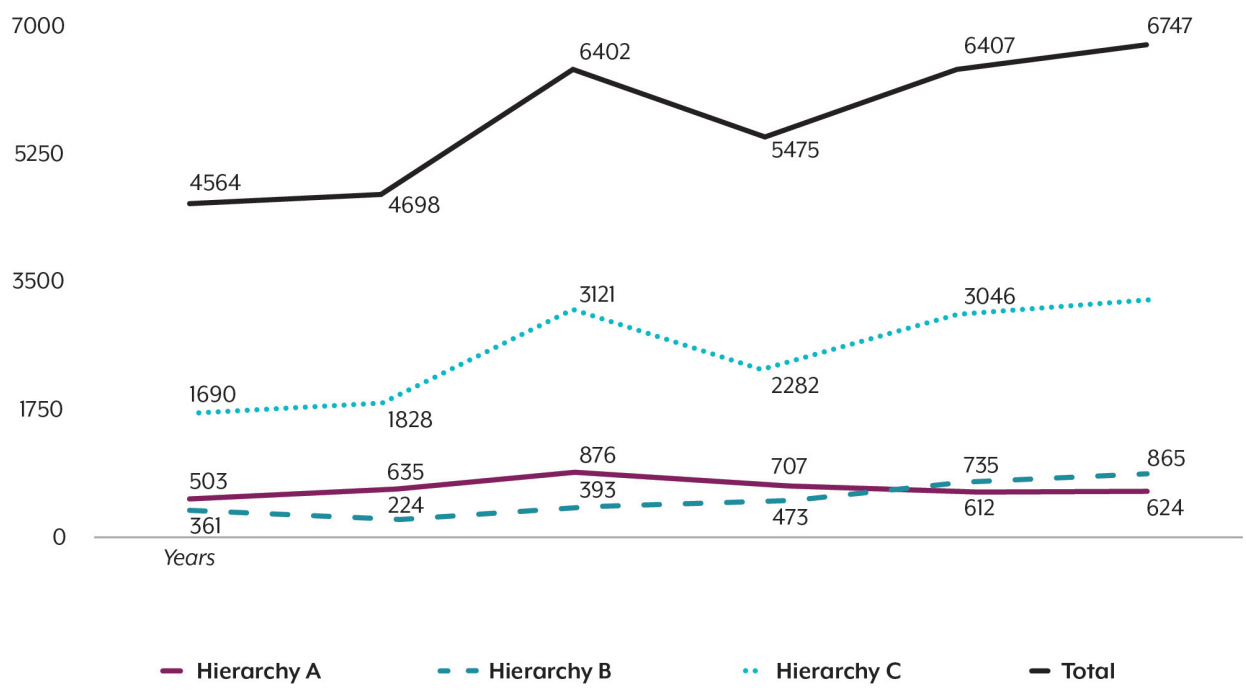

Figure 3.3: Distribution of graduates from 2010 to 2015 by staff category

- Hierarchy A applies to Physicians, Dentists, and Pharmacists. They have A-level exam + 6 years (Physicians) or 5 years of training.

- Hierarchy B is composed of Nurses, Midwives, Laboratory technicians, Public health technicians, and social assistants. They all have A-level exam +3 years of training.

- Hierarchy $\mathrm{C}$ is composed of Nurse-Assistants (ATS). They have O-level exam +3 years of training. 
Figure 3.3 shows an upward trend in the number of graduates (total 15,000 HWs) trained in Guinea between 2010 and 2015 and that ATs, state nurses, and midwives were the largest professional groups trained. Almost all professional categories (except ATS) were trained in lower Guinea, amounting to nearly $44 \%$ of the HWF trained over the last five years.

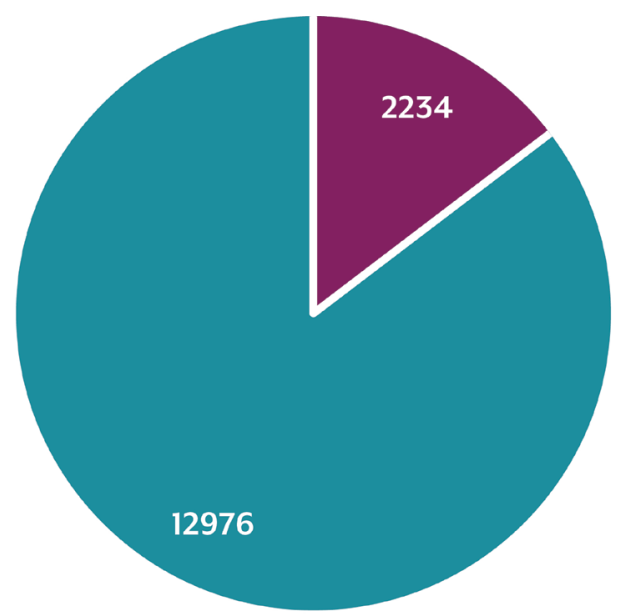

- Graduates from private training institutions

- Graduates from public training institutions

Figure 3.4: Distribution of graduates from 2010 to 2015 by type of institution (private or public)

Figure 3.4 shows the importance of private institutions in the training of certain professional categories between 2010 and 2015. Midwives, state nurses, and public health technicians were primarily trained in private institutions. Doctors, pharmacists, dentists, social assistants, and ATs were exclusively trained in public institutions.

\section{DISCUSSION}

The results provide an account of the challenges and possible solutions to improve HWF development in Guinea. The discussion builds on the results through analyzing different elements of the health policy triangle, followed by an analysis of the educational data, and finally some initial HWF policy, and governance recommendations and implications. 


\section{HRH situation and contextual factors}

It is challenging to employ and retain HWF in rural districts. The results indicate that the economic development situation makes it difficult for HWs to establish themselves permanently. They can only rely on a limited civil servant salary and there is less economic demand for additional private health services than in Conakry. Women HWs, generally about $60-70 \%$ of the global workforce, face particular challenges. International evidence points to systemic gender discrimination and inequalities in pre-service and in-service health education and (rural) employment. (Newman 2014) While not specially addressed in this study it is of imminent importance to take a gender and equity analysis in follow-up studies tracing HWF policy implementation in Guinea. (World Health Organization 2019a)

Respondents mentioned the need to be able to recruit and manage the HWF locally. Since salaries are paid at the central level, district-level health-team managers have no leverage to incentivize or sanction Hws' efforts. In general, it is crucial to find a good balance in dividing HWF governance responsibilities across central, regional, and district institutions. (Kolehmainen-Aitken 2004) Ongoing decentralization of government functions should facilitate this process.

\section{Workforce policy content and development}

The respondents indicated ongoing challenges in recruitment policies in the past and were cautious regarding the expectations of new policy developments. There are three main government policy frameworks that should enable HWF development in Guinea. The first concerns the recruitment of HWF in public services. (Ministère de la Fonction Publique 2016; Ministère de la Santé de Guinée 2015a) Although this policy has led to the actual recruitment of 3,802 HWs in 2017, this study has not been able to obtain an actual annual costed plan to finance this expansion of the workforce. The national health financing strategy to attain Universal Health Coverage (UHC) targets spending $15 \%$ of the total government budget on health by 2020 . However, it lacks a plan to attain this increase and provides no insight into the proportion of the budget allocated to workforce development. (Ministère de la Santé de Guinée 2015c) There is a considerable number of unemployed, Hws away from regular health services. (Jansen et al. 2014) While they are formally graduated, many have not received postgraduate training. Thoughtful planning and accompaniment will enable some of them to be integrated into the health system. It has been estimated that Guinea should increase its employed workforce by $17 \%$ annually to meet the standard it has set itself. While Guinea could be more ambitious in its scale-up of the workforce, it seems possible to make these investments within projected fiscal space available. (McPake et al. 2013) 
Secondly, the establishment of the ANSS aims to increase public HWF capacity for essential public health functions. In 2017, the ANss contracted and deployed 10 HWs per district to increase this core capacity. Part of this staff was later integrated into the recruited personnel by the MoH. It remains unclear whether ANss can still contract staff outside the wage bill in the future.

Thirdly, in the wake of the EVD epidemic, there is recognition that health issues must be addressed directly at the community level. The ministry, supported by organizations like UN Children's Fund (UNICEF), has begun to develop a community health workforce (CHWF) to serve as an interface, and hence increase trust, between formal health services and communities. (Ministère de la Santé de Guinée 2016) The government was inspired by the Health Extension Program in Ethiopia, where a cross-country community-based program was rolled out. (Banteyerga 2011) The future capacity to actually manage an increased HWF in the districts, including their performance and career development, will be a challenge. Managing and supervising such a mixed HWF requires specific competencies, resources, and training. Continuous training possibilities and peer-support are necessary to retain staff in remote posts. Respondents also suggest a system of rotations and scholarships schemes (for future specialization) to incentivize HWs to stay in rural areas. (Kolehmainen-Aitken 2004)

\section{Actors, positions, and collaborations on HRH policy}

The response to the EVD epidemic facilitated the emergence and presence of many 'new' actors, both nationally and internationally. ANSs, NGOs, and international organizations have been recruiting personnel at the district level creating a new staff 'mix'. The interviews indicate that there are challenges in aligning the workforce along common objectives. A first challenge is generational since districts have an existing group of often elder personnel that is now confronted with a new generation of younger staff, trained in Conakry, perhaps even with better remuneration. The position of the 'volunteers', basically unemployed HWs, in the health centers, requires similar attention. The results clarify that this cadre feels replaced by staff coming from outside while not been given a chance themselves to become civil servants. Both situations might create potential conflict situations that should be anticipated.

A second challenge is skills-mix. While there are proportionally more medical doctors in the workforce, international policies and donors urge for task-shifting and the development of midwives and nurses. (Buchan et al. 2013) Some international agencies mentioned that they focus on re-training and upgrading competences of ATS to have a proper midwife or nurse accreditation. This creates a new division of competences and tasks between the HWF and requires a clear framework on how 
primary healthcare teams function. A third challenge is possible tension between the village health teams initially engaged in addressing the Ebola outbreak and the CHWF deployed by organizations afterward. At the central level, the $\mathrm{MoH}$ has attempted to create a terms of reference for the CHWF profile. (Newman 2014) At the local level, there seems to be considerable variation in competences, remuneration, and profiles in the CHWF. The fourth challenge is related to remuneration and contracting. Several development actors said that they have the flexibility to recruit HWs on a short-term basis, often offering considerably higher salaries than the MoH. The remuneration differences and agreement on priorities require a strong district management team to coordinate different actors and programs. Nevertheless, workforce projections suggest that far more international support is needed in Guinea, Sierra-Leone, and Liberia to address their current training capacity weaknesses if acceptable levels of HWF are to be produced. A suitable investment on the part of 'an international community awakened to the global security threat' (Kluge et al. 2018) would be in supporting a significant scale-up of this capacity. (McPake et al. 2013)

\section{Educational developments concerning the HWF}

Interviewed actors acknowledged that there is no formal coordination matching the growing supply of graduated Hws in the more remote regions (Figure 3.3) with labor market dynamics. The prospect of having a job in healthcare services might be reasonable in the long term but the short-term prospects for newly graduated students remain limited. Private schools focus mainly on nursing and midwifery training. The directors indicate that the bottleneck is the actual availability of competent teachers, internship possibilities to enhance practical skills, and limited accreditation and supervision. Exposure to practical healthcare work is limited, impeding graduate quality and potentially impacting population trust. Capacity development in the areas of HWF management, professional education, institutional development, and leadership requires attention and investment. (Frenk et al. 2010; Nyoni 2008) The labor market mismatch requires reflection on student selection, management and actually capping the number of entrance candidates. (Jansen et al. 2014) The medical faculty of Gamal University has already imposed a student stop in 2016 to address the relative oversupply of medical doctors.

\section{Governance of the Guinean HWF}

HWF governance is a complex health systems function, bringing state and nonstate actors as well as different sectors together at multiple levels. The centralized management lines do not easily facilitate such a 'horizontal' approach. The respondents confirmed that in 2016, a new MoH-led HWF committee was established at the national level. This committee should technically be the governing body that 
analyses, oversees, plans, budgets, and evaluates several HWF policy actions. Ideally, all actors should buy-in to such a governance mechanism. An HWF committee could consist of representatives from several ministries, including the MoF. Workforce development should be aligned with fiscal and budgetary space to invest in the health sector. Although some international actors favored more sector-wide budget support, they demanded accompanying institutional reform of the $\mathrm{MoH}$ to rationalize health systems development, improve efficiency and cooperation with other institutional government partners. (ECORYs Consortium de Santé 2016)

Agood national HWF strategy and implementation plan should be based on evidencebased policy, labor market, and demographic needs analysis, budgeting, and include a proper monitoring and evaluation (M\&E) plan. Some countries including Sudan and Indonesia have developed an observatory to monitor HWF trends. (Dussault et al. 2016) This requires capacity and policy space at the MoH to plan, manage, cost and follow-up all actors adhering to one HWF plan. (World Health Organization 2012a) Leadership must acknowledge that HWF is an important development (and not only health) issue, so seeking alignment and support from the main political and societal actors is required, even involving them actively in the HWF committee. (World Health Organization 2012a)

\section{HWF development in Guinea}

The policy processes required to reform and develop the Guinean HWF in a sustainable matter is complex, using a range of interventions rather than a singlepolicy solution. A comparative study on HWF policies in four post-crisis settings indicated that these moments enable windows of opportunity for change and reform can occur but are by no means guaranteed-rather they depend on a constellation of leadership, financing, and capacity. (Witter et al. 2016)

Two main recommendations can be provided based on the research. The first is that it is essential that there is guidance, commissioned research, and space for policy adjustments by the MoH on the implementation of the different HWF processes in the country, most notably the deployment of HWF to rural areas. This could improve the fragile trust between the government, HWF, and communities.

The second recommendation concerns the need to initiate dialogue with all relevant national-level actors to provide a situational and comprehensive labor-market analysis of the HW situation, expanding professional education, financing, and various trends in the country. (McPake et al. 2013) There is also a need to construct an HRH governance mechanism with its terms of reference and division of tasks of relevant actors involved decided. 


\section{Limitations}

Some difficulties were encountered during data collection. Firstly, two educational centers could not be visited, so data concerning these establishments were not collected. Only HWF supply and needs have been assessed in this study, while the financial and demand aspects require a different, in-depth analysis. Secondly, two key resource persons were not available for interviews. This limited accessibility to the latest government health budget and its breakdown for the HWF. Similarly, it has been impossible to assess the financial contribution of development partners to the budget for supporting staff recruitment. Finally, the participation of a foreign researcher conducting interviews could influence responses and possibly constituted a bias for the study.

\section{CONCLUSION}

Health workforce development in Guinea requires a reform notably as there is a considerable mal-distribution of HWF between rural and urban areas. The weak state of the health system aggravated the EVD outbreak and led the government to initiate a plan to revitalize the health system and workforce. HWs were recruited in 2017 and deployed to rural areas for a minimum of 5 years. This study raises questions and challenges in terms of policy development, governance, HWF labor dynamics, and professional education aiming to achieve sustainable staff retention in rural areas. A longitudinal follow-up of this deployment will be undertaken to understand the structural drivers and policy options related to staff retention and to evaluate the policy implementation of future medicalization of primary care in the districts and impact on the quality of services and health outcomes. The EVD outbreak provided for a policy momentum to reform the HWF in Guinea. All actors involved share a responsibility to sustain that momentum and strengthen the health system. 


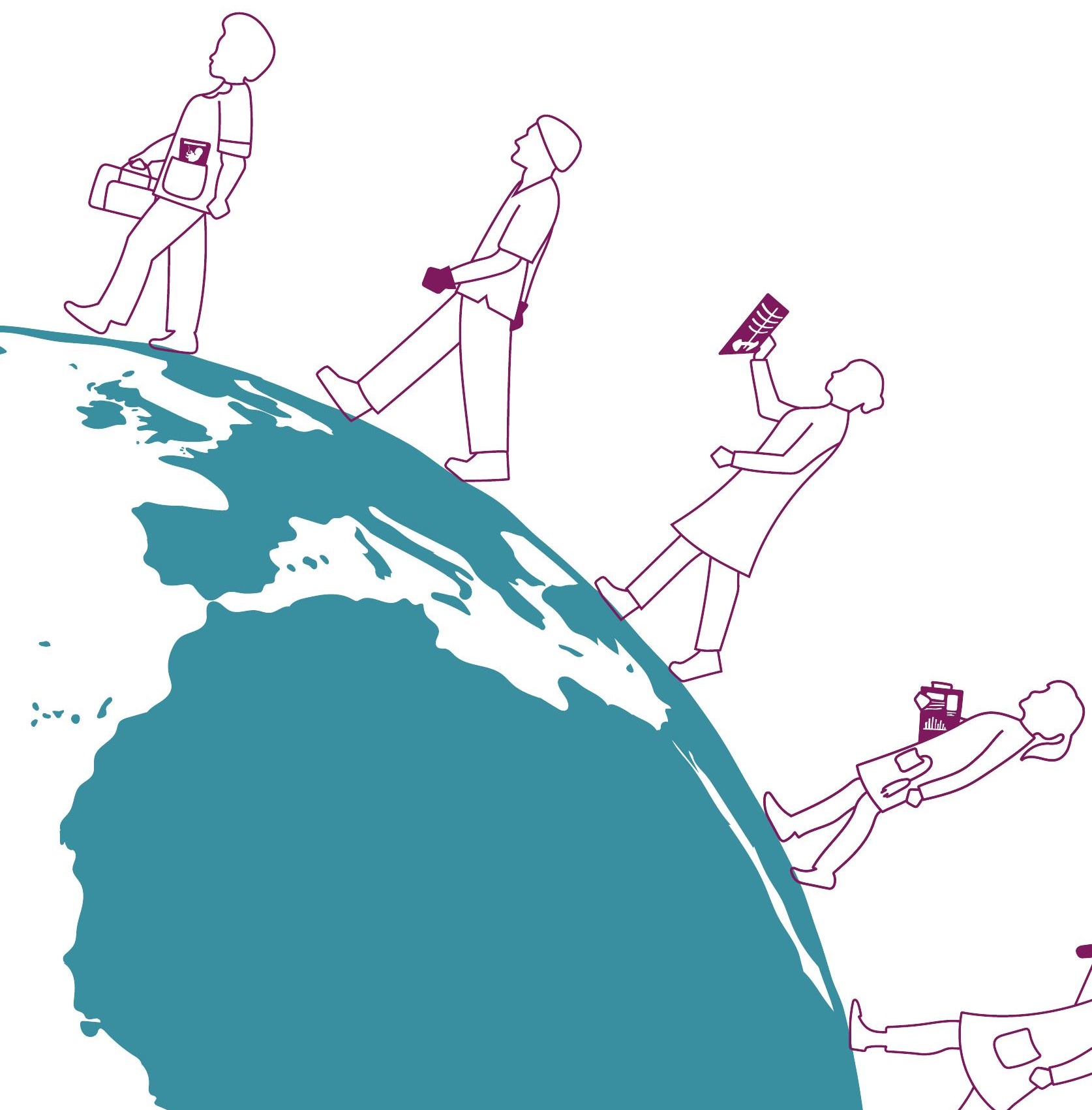




\section{2}

\section{Tracing the policy implementation of commitments made by national governments and other entities at the Third Global Forum on Human Resources for Health}

van de Pas, R., Veenstra, A., Gulati, D., Van Damme, W., \& Cometto, G. (2017). Tracing the policy implementation of commitments made by national governments and other entities at the Third Global Forum on Human Resources for Health. BMJ global health, 2(4), e000456.

doi:10.1136/bmjgh-2017-000456 


\section{ABSTRACT}

We conducted a follow-up analysis of the implementation of the Human Resources for Health (HRH) commitments made by country governments and other actors at the Third Global Forum on HRH in 2013. Since then Member States of the wHo endorsed Universal Health Coverage as the main policy objective whereby health systems strengthening, including reinforcement of the health workforce, can contribute to several Sustainable Development Goals. Now is the right time to trace the implementation of these commitments and to assess their contribution to broader global health objectives. The baseline data for this policy tracing study consist of the categorization and analysis of the HRH commitments conducted in 2014. This analysis was complemented in the application of the health policy triangle as its main analytical framework. An online survey and a guideline for semistructured interviews were developed to collect data. Information on the implementation of the commitments is available in 49 countries (86\%). The need for multi-actor approaches for HRH policy development is universally recognized. A suitable political window and socioeconomic situation emerge as crucial factors for sustainable HRH development. However, complex crises in different parts of the world have diverted attention from investment in HRH development. The analysis indicates that investment in the health workforce and corresponding policy development relies on political leadership, coherent government strategies, institutional capacity, and intersectoral governance mechanisms. The institutional capacity to shoulder such complex tasks varies widely across countries. For several countries, the commitment process provided an opportunity to invest in, develop and reform the health workforce. Nevertheless, the quality of HRH monitoring mechanisms requires more attention. In conclusion, HRH challenges, their different pathways and the intersectorality of the required responses are a concern for all the countries analyzed. There is hence a need for national governments and stakeholders across the globe to share responsibilities and invest in this vital issue in a coordinated manner. 


\section{KEY QUESTIONS}

\section{What is already known about this topic?}

Several country studies on advancing Human Resources for Health (HRH) commitments and national governance models have been published.

An overview study comparing different countries and tracing the implementation of this international health policy priority has not been conducted yet.

\section{What are the new findings?}

The study indicates that considerable progress has been made in several countries in implementing multiple HRH policies.

It also determines the contextual conditions and actors required to make this happen.

Moreover, it identifies gaps and issues that deserve attention in health workforce development.

\section{Recommendations for policy}

The HRH commitment process has engaged national governments and other actors in identifying, developing and implementing HRH policies.

This mechanism of action could be applied to other global health issues but attention is required to monitoring, accountability and financial aspects.

\section{INTRODUCTION}

In the lead-up to the Third Global Forum (3GF) on Human Resources for Health (HRH) held in Brazil (2013), countries and other entities were invited to make new HRH commitments to advance the HRH agenda. 57 countries and 27 other entities made commitments, which were announced at the 3GF. (Third Global Forum on Human Resources for Health 2013) These commitments were made when countries had to handle the repercussions of the global financial crisis, and several countries were acutely or prospectively facing conflict situations or public health emergencies such as the Ebola viral disease outbreak. Since then national governments have agreed on the Sustainable Development Agenda and the Member States of the World Health Organization (WHO) endorsed Universal Health Coverage (UHC) as the main policy objective through which health systems strengthening, including the development of an adequate, skilled, well-trained and motivated health 
workforce (HWF), contributes to several Sustainable Development Goals (SDG). (Marie Paule Kieny et al. 2017) The adoption of additional significant international policy frameworks supporting HWF development and the completion of the Global Health Workforce Alliance's (GHWA) mandate in 2016 presents a good moment to trace the implementation of the HRH commitments at the $3 \mathrm{GF}$ and to assess their contributions to broader global health objectives.

In preparation for the $3 \mathrm{GF}$, the GHWA and the WHO provided countries and other entities with a template for the identification of interconnected pathways and actions for systemic solutions to HRH challenges. This template recommended the inclusion of measurable targets to assess progress, and requested countries and other entities to be available for follow-up inquiries.

An initial analysis of the HRH commitments made by countries was conducted by the GHWA Secretariat in 2014. Commitments were categorized according to the Universal Health Coverage (UHC) framework of Availability, Accessibility, Acceptability and Quality and five areas of HWF action. (Global Health Workforce Alliance/WHO 2014) A clear demand from countries and international partners to follow-up on the commitments in order to ensure accountability and stimulate action for their implementation emerged.

Between February and June 2016, governments and other entities were approached in order to assess the implementation status of their HRH commitments and analyze the activities, policy mechanisms and drivers that facilitate HRH development at the national level. The objective of this tracing study is to analyze the implementation of the commitments by holding policymakers to account and by generating insights and evidence on relevance, effectiveness, and results of the HRH commitment process to date. The study provides insights into the pathways accelerating progress on the global HRH agenda, as well as an understanding of its main challenges.

First, this paper describes the execution of the study and the data collection. Then it looks at the factual outcome of the actions, including their monitoring, as implemented by countries. An overview of this is presented in boxes 3.2 and 3.3. Third, it provides a qualitative analysis of the contextual factors, the contributions by the different actors and the stages of the policy processes relevant for the implementation of HWF measures in a sustainable manner. Lastly, lessons learned and governance mechanisms and reforms required at the national and international levels to sustain the global HRH momentum are discussed. Exemplarily, several statements from the survey are presented in boxes 3.4-3.8 in order to enrich the analysis by illustrating the diversity of HRH actions taken by national governments and non-governmental organizations (NGOs). 
The baseline data for the policy tracing consisted of the HRH commitments analysis conducted by the GHWA secretariat in 2014. (Global Health Workforce Alliance/WHO 2014) Initially, a desk-based analysis consisting of a scoping review of the existing literature on HRH activities in each country was conducted. This review provided the reference against which subsequently the implementation status of the commitments, where available, could be verified. In the second part of the policy tracing study, the assessment of the outcome of the HRH actions was complemented by applying Walt and Gilson's health policy triangle as analytical framework. (Walt and Gilson 1994) The health policy triangle analyses policy development in terms of the interaction of actors, context and processes. In our study, the interaction between different actors, policy processes, and contextual factors driving the HRH activities was traced and provided insight into potential policy options for HRH investments and reforms. In addition, institutionalized and informal governance mechanisms, as well as the policy impact of international agencies on national HRH development, were assessed.

An online survey and a guideline for semistructured interviews were developed to collect data to be provided by the representatives from the governments and other entities (see online supplementary annex. 1). Triangulation of research data was performed via cross-checking available literature, policy documents and grey literature and through verification of the collected data by the wHO country staff.

\section{The implementation and monitoring of HRH commitments}

46 countries completed an online survey and/or interview explaining the status of HRH actions and related policy processes. For seven countries, detailed case studies on the implementation of the commitments have been published. (Dussault et al. 2016; Human Resources for Health Country Commitments 2015) Information on the implementation of the HRH commitments made at the $3 \mathrm{GF}$ is available for a total of 49 countries. No such information is available for eight countries.

For the 49 countries for which data are available, three countries responded being unable to implement the commitments due to a conflict situation and the impact of the 2014-2015 Ebola epidemic which also severely affected the HWF. 25 countries reported completion of the implementation for all commitments made or being in the process of doing so. 21 countries reported having partly implemented their HRH commitments, with conflicts or political instability listed as reasons hindering HRH actions. The categories and HRH pathways of the implemented commitments are generally in line with those of the baseline assessment from 2014. An overview of the implementation by country governments of their respective commitments is found in box 3.2 . 


\section{Box 3.2 results of the implementation of Human Resources for Health actions}

- 43 countries approached HRH challenges in a cross-sectoral way by implementing multiple commitments, such as investing in educational facilities for required cadres, accrediting training institutes, incentivizing practice in remote areas, developing institutional competencies in HRH management, strategic governance and information systems and labor market analysis (e.g., Panama, box 3.4).

- 36 countries' actions targeted improving the availability and accessibility of HRH. 27 countries have been working on quality improvement via the regulation of educational and professional performance (e.g., Cambodia, box 3.4),

- 17 countries have been working on the acceptability of HRH services, focusing on enhancing professional attitude and ethics via continuous professional development.

- 38 countries reported actions in the educational and training sector. Countries did not reform medical and nursing training curricula and introduced postgraduate and management training for $\mathrm{HRH}$ strategic planning, governance and monitoring, although progress regarding the latter shows considerable variation across countries (e.g., Bangladesh, box 3.4).

- 36 countries included labor market interventions, such as expanding the recruitment of different health cadres, regulating the qualifications of medical staff and quality of private clinics, increasing the budget for HRH investments, contracting private service providers, eliminating ghost workers from HRH registries and addressing absenteeism.

- Improving the skills mix, working conditions and professional autonomy in health services were prioritized in 20 countries, mainly in the WHO-AFRO and EMRO regions. These countries addressed this pathway by diversifying the education and recruitment of different cadres and investing more in community health workers and midwifery and in some cases nurse practitioners or clinical officers (cadres that provide first-line medical consultations and treatment).

- Systematic policy interventions at central governmental level represent other areas of focus in 43 countries. 26 countries have also implemented actions at the local/peripheral level, for example, providing policy space at district level to contract health workers, or to plan/manage the workforce according to local needs. 
- 16 countries, mainly in WHO-AFRO and EMRO regions, work on implementing community health workforce policies as a means to enhance the outreach and impact of health services.

- In 15 countries, mainly in the WHO-AFRO and PAHO region, Maternal, Neonatal and Child Health strategic objectives are important drivers for workforce development, especially by strengthening midwifery services.

- International migration of health workers and performance-based financing $(\mathrm{PBF})$ have not been identified as important drivers for policymakers, with only three and two countries working on mitigating international mobility and PBF, respectively.

- According to the original commitments, 17 countries had included HRH plans into a national health sector strategy or adopted an HRH plan. In 2016, more countries mentioned the (ongoing) development of a national strategic plan, the verification of which was difficult, as government approval was in some cases pending.

- Six countries had included a financial component in the original commitments and 23 countries defined measurable indicators to monitor their achievements. While tracing the use of indicators, these six countries did report on the progress in financing their workforce development while 20 countries used indicators to measure progress.

- In the African context, nine countries have improved their workforce register so that occupancy rates of posts, mobility of different cadre, as well as educational, recruitment and attrition data are regularly updated. The health labour market is covered only partially as these normally do not capture the workforce trends in private educational and health services.

Although HRH plans and strategies do exist for many countries, the availability of overview reports that monitor progress and evaluate these strategic plans as well as HRH commitments is limited. Several countries have shared annual and ad hoc reports of different formats. While absolute indicators are sometimes provided, such as '1,000 additional midwives have been recruited in 2015' denominators and baseline data frequently remain unclear. Likewise, the analysis of $\mathrm{HRH}$ policies as part of demographic and labor market trends is often neither available nor quantified by indicators. In the WHO-Pan American Health Organization (PAHO) region (e.g., Costa Rica, box 3.5) and in several countries in the wHO-South-East Asia Region (SEARO) region, these reports are more structured. Online HRH information and monitoring platforms have been created (e.g., Ghana, Republic of Moldova, box 3.5) and are sometimes linked to national or regional HRH observatories. 


\section{Policy mechanisms and HRH governance}

Some broader trends regarding policy mechanisms (the interaction of actors, context and processes) relevant for the implementation of the commitments as well as HRH governance modalities were assessed by a qualitative analysis, complementing the quantitative analysis of the HRH actions. In all responding countries, there was recognition of the need for multistakeholder approaches for HRH policy development. In most of the countries, scaling up the numbers of employed health workers was considered a priority over education. Their retention and equitable distribution were also considered important. Many countries recognized the need to adopt diverse skills mix models and increase the training of mid-level cadres, such as village midwives. Moreover, the need for investments in capacity building for HRH policy development and management was prioritized in several countries.

In 32 countries, basic HRH governance mechanisms such as technical working groups or HRH strategic committees exist-mostly under the management of the ministry of health. In countries with a strong presence of external agencies, HRH meetings regularly take place as part of donor co-ordination programs. Particularly in those countries, in which health and labor market development is a government priority, HRH governance bodies are broad in scope and formally backed by a highlevel institution. In other countries, the approach involves fewer actors and is more technical. A few international NGOs have played an important role in strengthening technical and governance capacity. GHWA's Country Coordination and Facilitation model has been used to develop and manage the HRH governance mechanisms of Indonesia, South-Sudan and other countries in the recent past. (Global Health Workforce Alliance/wHO 2012; 2013a; Kurniati et al. 2015) An overview of the initiated policy and governance mechanisms can be found in box 3.3.

Respondents noted the momentum that the $3 \mathrm{GF}$ commitment process provided, contributing to domestic and international recognition and, sometimes, investments in workforce development. Some stated that the HRH plans were already part of an existing government strategy while in other cases commitments initially developed for the 3GF were subsequently adopted and incorporated into national planning and strategic frameworks (e.g., Ethiopia, box 3.7). The examples in boxes 3.4-3.8 (but not exclusively) indicate that in a range of countries considerable advancements in scaling up a workforce fit-for-purpose have been made. Also, the Western-African countries affected by the Ebola viral disease outbreak in 2014-2015 have used this moment of crisis to rapidly scale up the workforce with the aim to strengthen the resilience of their health systems. (e.g., Guinea, box 3.7) Generally, respondents found the commitment process and subsequent follow-up to be a useful mechanism and encouraged such models to be repeated in the future. 


\section{Box 3.3 results of the initiated policy and governance mechanisms}

- The respondents of 40 countries mentioned the importance of having a department within the Ministry of Health that is in charge of harmonising activities across the remit of different ministries, such as the ministries of education and finance (e.g., Burkina Faso, box 5). Respondents mentioned that often co-ordination exists with the ministries of social, labour and internal affairs (e.g., Indonesia, box 3.6).

- In five countries, health workforce developments have attained sufficient importance for general cabinet and senior ministers to be involved in policy deliberations, decision making and follow-up of implementation (e.g., Sudan, box 3.6).

- Universities and other training institutions, professional associations and, to a lesser extent, community-based organisations are important actors in national Human Resources for Health (HRH) governance. In 27 countries, multilateral agencies (WHO, UNICEF, United Nations Population Fund (UNFPA)) and development banks play an important (technical) role. NGOs and bilateral donors can thus play a substantial role, reflecting the relevance of international assistance particularly in countries of the WHO-AFRO region and fragile states (e.g., Intra-Health International, African Medical Research Foundation (AMREF) Health Africa, box 3.8).

- The interviews indicate that upper-middle income countries exhibit stronger HRH departments and government ownership of the HRH strategic agenda, while lower-middle and low-income countries rely more on the interest and financing of external actors. In 13 countries within the WHOAFRO region, HRH development is a combined priority for the government, NGOS and funding agencies. In other regions, the picture is different, with a comparatively more limited role for international actors in the WHO-PAHO, SEARO and EMRO regions.

- 22 respondents support the role of international agencies, global health initiatives and NGOS in HRH development. However, several countries have highlighted declining interest in health systems strengthening due to the global recession and its impact on funding, particularly from European donors. Moreover, competing activities by global health initiatives, such as the Global Fund, Global Vaccine Alliance (Gavi) and the US President's Emergency Plan for AIDS Relief (PEPFAR), and international NGOs are sometimes considered a problem.

- According to respondents, neither labour unions nor private commercial health service providers have a noteworthy role in HRH governance in most settings. 
Box 3.4 Examples of actions according to the Availability, Accessibility, Acceptability and Quality dimensions and policy pathways

- The Ministry of Health succeeded in improving the quality of preservice education, developing a licensing and registration system for the health workforce in order to improve the quality of care services. The development of a new health workforce development plan, including strategic objectives and interventions, has been defined as part of the government's strong political commitment to strengthen the competency and regulation of the health workforce. 'The HRH commitment process helped provide additional political support towards this end' (Cambodia).

- The government implemented several Human Resources for Health (HRH) actions couched within a comprehensive HRH plan. For example, updating the recruitment rules for different cadres and institutions. Continuing medical education is undertaken by the Centre for Medical Education, the capacity and content of nursing training and education was expanded, and an accreditation system was institutionalised within the Ministry of Health. 'The initiatives ...which were undertaken for developing the commitments and implementing them create[d] momentum to put emphasis on the HRH agenda at all levels' (Bangladesh).

- The government has worked progressively on the formation of regional teaching units, created as a means for strengthening health services and development of human resources by implementing processes of health education, identifying training needs and co-ordinating national $\mathrm{HRH}$ development in broader processes. Training workshops were held, in which multiple actions were identified in order to strengthen regional teaching units, such as the development of a training programme of Primary Health Care for rural areas in Panama (Panama). 


\section{Box 3.5 Examples of monitoring mechanisms being initiated}

- The Ministry of Health (MoH) committed to strengthening management in the Human Resources for Health (HRH) department by 2020. They have developed and presented a proposal aimed at improving HRH management. Staff members were trained to improve their capacities to manage HRH policies. A national HRH observatory where data on health professionals are collected and updated annually related to general information, training and employment has been functional since 2012. The actions and commitments have been successful due to strong and sustained political commitment and institutional capacity (Costa Rica).

- In addition to the original commitment to develop and implement an HRH Commitment Action Plan, the state has made a lot of progress towards $\mathrm{HRH}$ development. To overcome inadequate numbers and maldistribution of health workers, a staffing norm was designed and implemented. A Human Resources Management Information System has been piloted and will be launched with the objective of managing and tracking the health workforce (Ghana).

- The government has initiated a functional national monitoring mechanism for HRH information and evaluation; this monitors among other aspects employment of newly graduated health workers in the health labour market, incentive structures, accreditation and curriculum standards, competence of nurses authorised for core health service tasks, and the number of bilateral and multilateral agreements in force (Republic of Moldova). 
Box 3.6 Examples of Human resources for Health governance mechanisms being initiated

- The HRH thematic committee under the auspice of the Directorate of Human Resources engaged in a multistakeholder approach involving all actors relevant for HR management. Among other actors, the Ministries of Health, Finance, Labour and Social Security and Public Service cooperated to improve the quality and availability of Maternal, Neonatal, Child Health (MNCH) cadres particularly in underserved regions. The ' ... (commitment) process has shown that concrete and operational actions could be identified and implemented to improve the distribution of and the quality of care of MNCH HRH in a concerted manner by seeking consensus of all stakeholders'(Burkina Faso).

- The HRH governance mechanism is part of a national Health-In-All-Policies strategy. This is managed by a National Council for the co-ordination of health services that is directly headed by the President of the Republic, who is committed to health development. $\mathrm{HRH}$ is a subtheme for which there is a special committee. There is governmental willingness, multi-actor dialogue and international assistance to develop the workforce. 'The issue of governance is very important. One has to ensure that there are strong governance tools' (Sudan).

- We expect to include almost all population by 2019 in a Universal Health Coverage scheme. This is a strong political commitment in this country to ensure everyone has equal access to quality health service. Yet, we still face various challenges including shortage of health workforce especially in remote areas. Main priority should be addressed to overcome those challenges by working with the local government. HRH actions, including the Coordination and Facilitation governance mechanism, should receive strong commitment from all stakeholders to support the fully functioning of the health system in every areas of the country' (Indonesia). 


\section{Box 3.7 Examples of the Human resources for Health political momentum}

- As part of the revival of the health system (after Ebola), the state undertook to recruit 2000 health workers who will be transferred in priority to disadvantaged areas. During the process of recruiting these 2000 health workers, each candidate signed a commitment to serve at least 3 years in rural areas. 'Due to political will as well as reflecting on the socio-economic impact of the Ebola epidemic, we have agreed on a new national health policy and health development plan that incorporates strong participation of Community Health Workers' (Guinea).

- 'Following the Recife declaration a situational analysis was made and strategic documents were prepared. This created a real momentum to employ new graduates and to an expansion of medical and health science colleges. Also, the ministry has recently started an international institute for Primary Health Care (PHC) for south-south cooperation between African countries on PHC development' (Ethiopia).

\section{The importance of engaging with non-governmental actors}

$16(59 \%)$ of the other entities-NGOs, professional associations and (regional) institutions providing technical assistance responded. Their commitments differed considerably and were sometimes highly specific. Several entities have funded and initiated integrated HRH policies and activities in partnership with governments. Only a few international NGOs have the resources, skills, and competency to cooperate on HRH development in multiple countries. These NGOs have played a key role in the development of the original country commitments as well as their implementation in partnership with governments. Other NGOs have used the commitments for their advocacy for sustained HWF investment. Some NGOs focused on digital innovation and skills transfer to strengthen nursing and midwifery. Seven NGOs have focused on investing in community health workers (CHW) development. Professional associations provided technical support to develop plans and strategies, while regional institutions and observatories provided a platform for the harmonization and standardization of HRH actions among countries (e.g., Health Schools network of the Union of South American nations, International Council of Nurses, box 3.8). Other organizations have used the commitment framework to highlight their own HRH work and to effectively guide HRH activities, monitoring, advocacy, and accountability towards donors and partners (e.g., the Health Workers 4 All project, box 3.8). 


\section{Box 3.8 Examples of Human resources for Health actions by other entities}

- We committed to contributing to strengthening the network of national HRH leaders around the globe. Over the last two years, we have actively engaged senior HRH leaders in more than 15 countries in provision of technical support, training, and leadership development regionally, nationally, and sub-nationally. Further, we continue to maintain the HRH Global Resource Center as a go-to reference site for information on the field of HRH' (IntraHealth International).

- The Executive Secretary develops a series of meetings with training institutions to identify the possibility of conducting more regional or (sub)regional courses on HRH management and governance' (Health Schools network of the Union of South American nations).

- In multiple countries the HRH priority is to match the community needs for health workers with the skills and competencies that are being taught at the health training institutions. A key priority is getting a mono-technic midwife in rural Africa a priority as compared to ensuring access to a multi skilled nurse midwife' (AMREF Health Africa).

- We have been active in advocacy, clinical practice, international migration, career development, leadership roles in health systems and a wider range of other workforce issues' (International Council of Nurses).

- We have been advising and urging policymakers at EU and Member State level to develop and maintain strong health systems and sustainable health workforces both within Europe and elsewhere. A main lesson learnt is the confirmation that the multi-stakeholder approach promoted by the Global Code of Practice on the International Recruitment of Health Personnel is key to its implementation' (Health Workers 4 All).

\section{Lessons emerging from the HRH commitment process}

It is important to acknowledge some of the limitations of this analysis by contextualizing the findings: data collection took place only two and half years after the 3GF while intersectoral HRH policy implementation and workforce development require time. Countries have set goals for their activities, but a timeframe and related milestones were missing for most commitments. This affected the possibility to systematically assess and compare progress in commitment implementation. 
Notwithstanding the aforementioned caveats, the results indicate that 46 countries are progressing towards their commitments. Countries have mainly focused on the implementation of policies to incentivize retention in rural areas, investing in and regulating education, professional accreditation and the development of a CHW cadre, and capacity building on management, monitoring, and governance. Gender mainstreaming, skills-mix reforms and international migration received limited attention.

\section{An enabling context for HRH actions}

Investment in the HWF and policy development was enabled by strong political leadership and coherent government strategies. The actors involved benefitted from sufficient institutional capacity and collaborated coherently within functioning intersectoral governance mechanisms. The 57 countries vary substantially regarding their institutional capacity to conduct these complex tasks. Some countries used the momentum of the $3 \mathrm{GF}$ to accelerate HRH policies previously agreed on as part of national strategic plans, while others have used the commitments as key principles guiding HRH policy development

In these countries, the problem, policy and politics streams converged to create a window of opportunity for countries to invest, develop and reform the HWF.

A suitable political window and socioeconomic situation emerge as crucial factors for sustainable $\mathrm{HRH}$ development. A stable sociopolitical situation and economy are required for the government to create demand and expand its investments in the workforce. Several Low- and Middle-Income Countries (LMICs) throughout all regions have over the last years been able to harness such momentum. Most of these countries experienced considerable economic growth, while no examples of (countercyclical) government investments in workforce development during an economic downturn can be provided. Moreover, complex crises (armed conflicts, climate disasters, displaced populations, financial crises, and epidemics) divert attention from HRH development and investments. Nevertheless, over the last years, several transnational viral disease epidemics have also raised awareness of the need to strengthen global health security-including the HWF as a crucial component. (The Lancet - Editorial 2016)

\section{Key actors for HRH development}

Our findings indicate that international agencies and international NGOs play a considerable role in advancing HRH processes at both the global and national levels in some nations. Countries in the PAHO region have benefitted from strong political 
support and intercountry technical cooperation for HRH policy development at the (sub-) regional level, including by the PAHO office itself. (Dal Poz et al. 2015; Pan American Health Organisation 2005) In the Western African Region, funding provided by France in the context of the G8 Muskoka initiative provided important financial support to develop and implement $\mathrm{HRH}$ actions in the field of maternal and child healthcare. (G8 Communique 2010) ${ }^{13}$ In multiple African countries, close cooperation with key international NGOs contributed momentum and funding for HRH actions. (Amref 2014; Human Resources for Health Country Commitments 2015) Reliance on external funding and support for HRH raises questions about sustainability and domestic ownership of the HRH agenda. However, our assessment indicates that HRH governance and development have been awarded higher priority by governments in the countries analyzed following the $3 \mathrm{GF}$.

Intersectoral policy development to expand fiscal space for workforce investments remains a challenge. Financial targets have only been included in the commitments of a few countries and it is unclear whether this has led to corresponding budgetary adjustments by governments. As the public sector's fiscal space is closely aligned to broader governmental strategies and political choices, progress in expanding and financing the wage bill is slow in most places. However, when there is political support from government leaders for the crucial role of the HWF in contributing to broader health, economic and employment objectives in society (this has been the case in Ethiopia, Ecuador, Indonesia, Sudan, Ghana and the Republic of Moldova), a rapid scale-up of HRH investments is possible.

The other entities, notably some international NGOs, have been instrumental in developing and implementing $\mathrm{HRH}$ actions in partnership with country governments, especially in a range of Sub-Saharan African countries. These partnerships have enabled funding and momentum to put policies and innovative solutions such as E-Health training modules and mobile health applications in place. A number of organizations have developed (and want to harmonize) the CHW program. Although some countries have developed CHW strategies, the work of NGOS is often complementary to that of the government when it comes to the training and remuneration of this cadre. This could create some tension regarding the sustainability, prioritization, and integration of these programs. (International) professional associations and networks have been relevant for strengthening norms in competences and training. However, their role in the governance of national HRH mechanisms seems limited. Lastly, it can be noted that labor unions and the commercial private sector have rarely been mentioned as key actors in governance mechanisms. 
The commitment process demonstrated that HRH observatories do play an important role in monitoring and evaluation of $\mathrm{HRH}$ development and policies. There are positive examples of regional cooperation in HRH observatories and integrated HRH information systems, especially in Latin America, Europe, and South-East Asia. It is recommended for all countries to establish institutional mechanisms and processes, such as an HRH observatory, working groups and/or HRH co-ordination committees in order to expand the evidence base and to promote policy dialogue on HWF issues as well as holding all actors accountable concerning HRH policies and actions initiated.

\section{Advancing the international health workforce policy process}

The commitment process generated political support and momentum to invest in the HWF. Most of the 57 countries that had made commitments at the $3 \mathrm{GF}$ faced severe HRH challenges. The multipronged, cross-thematic approach chosen by many countries indicates deepened knowledge of the governance mechanisms required to deal with this complex issue.

Notwithstanding, some caution is warranted: HRH planning and management require a long-term perspective. The momentum that has been generated by the 3GF must not be lost. Political instability and 'shocks' such as epidemics, financial crises, and environmental disasters could disrupt those earlier investments but could also raise political awareness of the need to support countercyclical economic investments to strengthen public health systems and public services employment.

The commitments were generally lacking appropriate financial planning and indicators to sustain HRH developments and to monitor success in the long run. This likely is the case due to a fear of cost escalation. However, the commitment process and the Recife Political Declaration on HRH (World Health Organization 2013a) provided the space to give HRH development and UHC priorities due consideration in discussions on the Sustainable Development Agenda. Consecutively, highlevel political attention by the United Nations High Level Commission on Health Employment and Economic Growth (UNHEEG commission) has highlighted the potential contribution of health employment to equitable economic growth and may add political momentum to sustained investments in the future workforce. (World Health Organization 2016d) The tracing of the HRH commitments also highlighted the continued need to develop strong monitoring and evaluation (M\&E) mechanisms at the national, regional and global levels for greater accountability.

The SDGs include a specific target $3 c$ for HWF Development, and the HWF can contribute to the attainment of other SDGs. (Tim Evans et al. 2016; World Health Organization 2015b) In 2016, the World Health Assembly adopted the Global 
Strategy on HRH: Workforce 2030. (World Health Organization 2016a) The UNHEEG commission has invited policymakers to commit to its agenda and the 10 recommendations it has put forward. (World Health Organization 2016f) The SDGs, the global strategy on HRH and the UNHEEG commission provide the policy guidance and political framework on how to further the ongoing national and global HRH commitments.

The HRH commitment process provides national and regional examples of intersectoral and multiple actor governance, including its policy dialogue, accountability, monitoring and observatory functions. It demonstrates that similar functions need to be secured at the global level. Global HRH development has become an international public good that is required to improve universal health outcomes, facilitates decent employment and represents a crucial pillar for health security. HRH development demands sharing responsibilities and political commitment as well as investment by countries and other actors to overcome the global gap in workforce shortages.

These factors merit policy proposals and dialogue on the initiation of a governance mechanism at the global level that monitors HRH investments, overviews country progress in the different HRH policy pathways linked to the WHO Global Strategy On HRH and the UNHEEG commission, monitors the WHO's Code of Practice on the international recruitment of health personnel and provides a forum for policy dialogue on managing transnational workforce mobility. Such a global platform would facilitate exchange, communication, best practice and mutual accountability between countries and other actors regarding HRH developments, and would act as a nexus for intersectoral and structured dialogue with other global mechanisms such as the UHC 2030 alliance, the High Level Political Forum on Sustainable Development, G7/G20, Multilateral, and regional trade agreements, the International Financial Institutions, International Labour Organisation (ILO)'s decent work agenda, the International Health Regulations and the Global Health Security Agenda. The Global Health Workforce Network is an excellent place to discuss the potential of such a mechanism, linked with and contributing to the SDG monitoring and accountability framework. (Berland et al. 2016)

\section{GONCLUSION}

The findings and analysis from the HRH commitments implementation indicate that intersectoral action, dedicated political support, a partnership approach, and sustained funding are of crucial importance to further advance the HRH development agenda towards the three objectives of equitable health outcomes, inclusive, and 
sustainable economic growth and improved health security. HRH challenges, their different pathways and the intersectorality of the required response are increasingly recognized as an issue of common concern; hence, there is a need for national governments to continue to share responsibilities and cooperate on this vital issue in a co-ordinated matter with all relevant actors. 


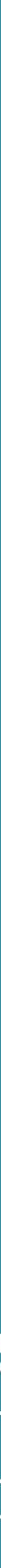




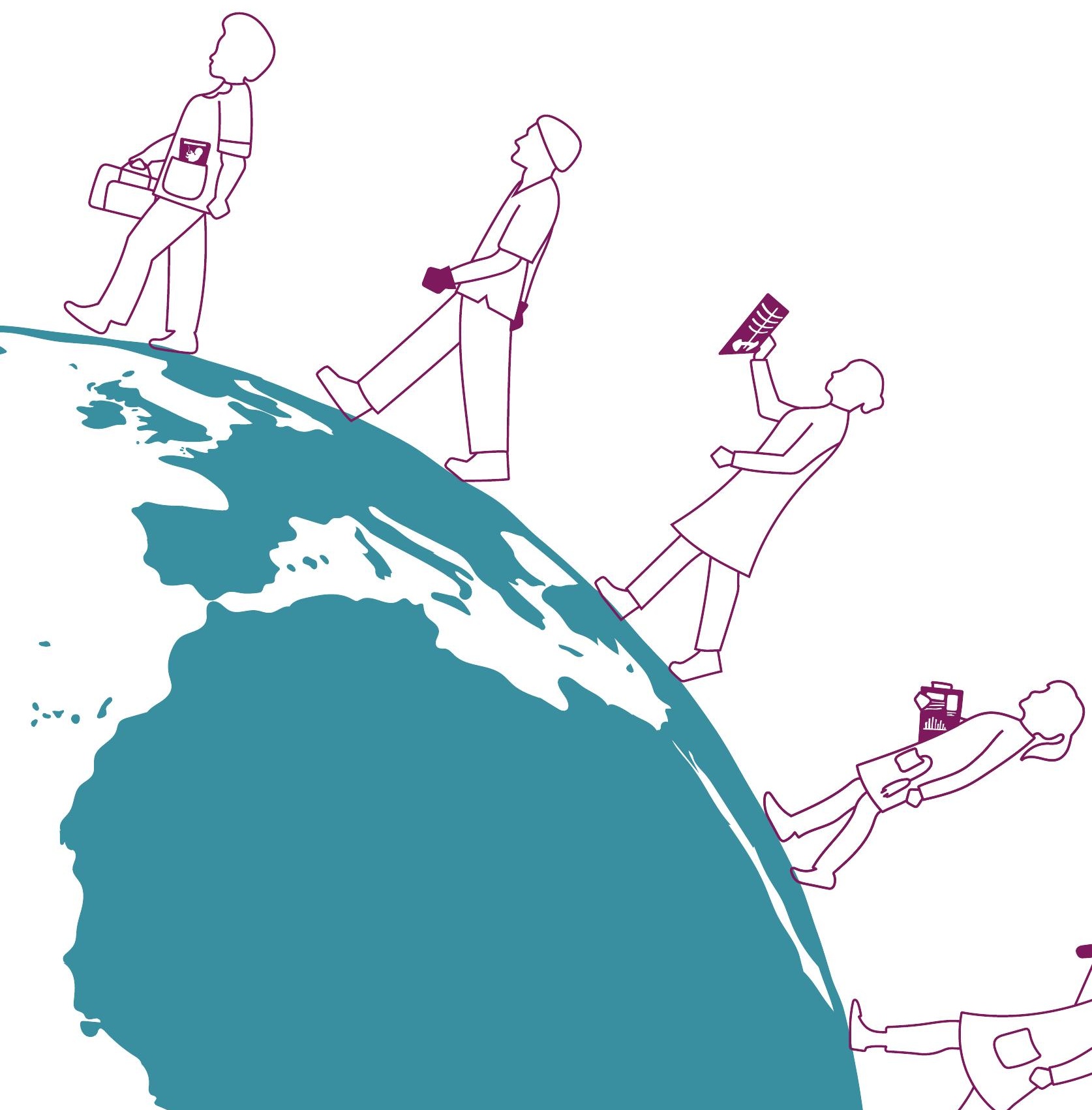




\section{1}

\section{Democratizing the World Health Organization}

Van de Pas, R., \& van Schaik, L. G. (2014).

Democratizing the World Health Organization.

Public Health, 128(2), 195-201.

doi: 10.1016/j.puhe.2013.08.023 


\begin{abstract}
A progressive erosion of the democratic space appears as one of the emerging challenges in global health today. Such delimitation of the political interplay has a particularly evident impact on the unique public interest function of the World Health Organization (WHO). This paper aims to identify some obstacles for a truly democratic functioning of the UN specialized agency for health. The development of civil society's engagement with the wHO, including in the current reform proposals, is described. The paper also analyses how today's financing of the wHO-primarily through multi-bi financing mechanisms-risks to choke the agency's role in global health. Democratizing the public debate on global health, and therefore the role of the wHO, requires a debate on its future role and engagement at the country level. This desirable process can only be linked to national debates on public health, and the re-definition of health as a primary political and societal concern.
\end{abstract}




\section{INTRODUCTION}

This article focuses on the need for democratizing the World Health Organization (WHO) and the public dialogue around health. Is the wHO functioning democratically today? First of all, this question requires some clarifications on how democratic legitimacy is actually defined in relation to the wHO's functioning. (van Ham et al. 2013) Secondly, we have to separate the democratic functioning of the wHO itself, from the current functioning of the global governance for health in which the wHO has a substantive role. This article mainly focuses on the first issue, and will only touch upon the latter.

Authors have diagnosed 'a deficit of democracy' as one of the key challenges for the WHO, as well as for the wider governance of global health. (Frenk and Moon 2013) It is one of the reasons that the wHO's work on human rights and health equity has been hampered over the last decades. (Lee et al. 2007) What has changed in recent times, in line with global trends in other sectors, is the mounting concentration of power-and money-when it comes to the bare handful of key decision-makers in global health. While the wHO is still functioning as a member state-driven multilateral organization, it is subject to a trend in which global governance has become polycentric and states have lost authority. (Scholte 2004) Is it then possible for the wHO to regain its multilateral legitimacy, through enhancing the quality of its democratic interplay in decision making? Can the wHO really be the key health authority in a globalized world based on a cosmopolitan democracy? And what would be the incremental steps required for this? (Koenig-Archibugi 2010)

\section{The democratic legitimacy of the wно}

Democratic legitimacy in transnational governance arrangements can be conceived as a five-faced prism, whose surfaces are respectively: (1) representation; (2) accountability; (3) transparency; (4) effectiveness; and (5) deliberation. (van Ham et al. 2013) Before we address these different faces, a fundamental contradiction in contemporary multilateralism requires explanation.

The wHO, like other United Nations (UN) institutions, has been created to enhance cooperation between states on issues of security and welfare (e.g. Polio eradication). As this cooperation is more effective than action by states alone, this creates output legitimacy. This should be complemented by input legitimacy, which implies the diversity of representation and inclusiveness of all its (sovereign) Member States.

For example, an international convention, such as the Framework Convention of Tobacco Control (FGTC), is so powerful because it is a diplomatic negotiation 
between 194 Member States and because it has included deliberation with non-state actors such as civil society. (Lencucha et al. 2010) This ideal vision of democracy is tarnished by a contradiction between the nominal state-egalitarianism of multilateral organizations and the realities of power politics, where weaker states may be unwilling to defy their powerful neighbors, creditors and trade partners. Moreover, many countries in the UN system are undemocratic or only partial democratic and their positions in the UN do not necessarily represent the interests of its citizens. Multilateral organizations are not organized democratically-with equal votes for each individual-but on a statist basis.

Principles of state sovereignty, whose origins lie in monarchy, and democratic policy-making are conflicting. Within the 21st century, the ideology of democratic governance makes it harder to organize the world on the basis of sovereign states. In democratic theory, individuals, not states are the subjects of political and moral concern. (Keohane 2006) There is hence a demand for UN institutions to adjust their governance models by improving its input legitimacy that goes beyond state representation. This could imply the inclusion of 'extended state' representatives that in the views of Antonio Gramsci includes not only the political sphere but also exists of, and is closely linked with civil society. Both within states and within multilateral organizations, this 'extended state' can contribute to the democratic legitimacy of policies. (Gebauer 2012)

\section{Representation (inclusiveness)}

The WHO remains in today's globalized world the one 'directing and coordinating authority' for the realization of the right to health and universal coverage: A role that is tightly embedded in its Constitution. (Medico International 2011) The WHO Member States have a legal responsibility for the health of their citizens. Currently, the wHO consists of 194 Member States. This includes tiny states such as Monaco as well as a giant country like China.

Over the recent years, the WHO's formal governance bodies, the World Health Assembly (WHA) and Executive Board (EB), have become more transparent and accessible, both for Member States and non-state actors. (World Health Organization 2012d) One of the positive effects of the current wHO reform is that countries become better prepared to the meetings. Diplomatic cooperation between Member States has become more intense. For example, since the European Union (EU) has a formal foreign diplomatic service (2010), it also has a formal delegation to the WHO'. The EU delegate facilitates the EU 28 members to come to a joint position on the

1 The EU has an observer status at the WHO. It speaks with 'one diplomatic voice' during the EB and WHA via its half-year rotating member state presidency. 
WHO policies. (van Schaik and Battams 2014b) As a result, other regional economic integration bodies, such as the Union of South American Nations (UNASUR) and the African Union (AU) also internally consolidate their positions on the WHO's policy. There is a growing interest in the role of the 'BRICS' (the emerging economies Brazil, Russia, India, China, South Africa) in global health and the wHO. The two BRICS health ministers meetings so far have identified shared global health priorities such as non-communicable diseases (NCDs) and cooperation for Research and Development. Despite robust interventions of India and Brazil at the WHA, the BRICS have not yet spoken out en bloc at the WHA or EB, and rather focus their diplomatic efforts on the G20. (Harmer et al. 2013) Countries start to take the wHO and global health more serious within their foreign policies and have created dialogue and space with its domestic stakeholders to prepare its position for the WHA and EB. (Gagnon and Labonté 2013)

However, engagement by Member States in the wHO's policies and its governance structures remains limited in general. The WHO's governance system is considered archaic, while the policies of the organization, including appointments of strategic positions, are politicized and determined by its main donors. (Chow 2010) At the WHA in 2013, it has been noted by some Member States that 'governance has been the most neglected area of the reform process', especially when it comes to the wHO's relationship with external actors. (Kamradt-Scott and Sangiorgio 2013)

\section{Inadequate finances and a lack of transparency and accountability}

Looking back at the wHO history, one realizes that Member States have not always done the agency a very good service. For example in 1984, in response to the perceived politicization of the UN organizations in the late '70s, the so-called Geneva group (comprising the 11 major donors of the UN agencies, including the United States (US) and several European states) set out to restrict the growth of international agency budgets, including the wHO, to zero in real terms. (Clift 2013) In the case of the WHO, this policy was further sharpened to nominal zero growth in 1993.

Defacto, just as the Health for All policywas to be enacted after the Alma Ata declaration in 1978, the agency started to be choked and bereft of its financial capacity and potential development. Today, this deprivation has become a structural condition, and WHO has lost control over its budget, hence over its institutional autonomy. The vast majority of the funding to the agency is provided via extra-budgetary voluntary contributions that-through the WHO-actually serve the interests of particular state and non-state donors. The Organisation for Economic Co-operation and Development (OECD) has phrased this development muIti-bi financing. (OECD/DAC 2010) Through this increasing trend, participating governments and others are 
controlling international agencies more tightly, thereby impacting on their policy priorities. (Sridhar 2012) In it's the WHO-strategy 2011-2015, Sweden argues that the wHO's legitimacy is undermined by accountability issues regarding the allocation of resources. Budget control was found to be weak and operations only partly governed by decisions of the WHA and EB. (Sweden 2011)

Funding for global health has grown significantly over the past decade, from USD 5.7 billion in 1990 to USD 27.73 billion in 2011. (Institute for Health Metrics and Evaluation 2011) This money has largely bypassed the wHO, possibly because of donors' lack of confidence in the agency. (Butler 2011) The current trends demonstrate that most funding has shifted to development assistance for global health. The wHO's core public health policies and norm-setting role risks to remain underfunded.

\section{Effectiveness (decisiveness)}

The role of the wHO at the national level is often weak, for different reasons. The focus of the WHO's activities in countries is primarily technical support to governments. Evaluation of programs is weak, resulting in a lack of insight if resources are spent effectively and efficiently. For Member States, which use taxpayers' money to fund the organization, this is hardly satisfactory, and therefore they demand a greater degree of transparency and accountability during the ongoing effort to reform the wHO. (Department for International Development - UKaid 2011)

The wHO's effectiveness is inherently hampered by the fact that its official guidance is derived from 194 Members. Resolutions and treaties by the WHA and EB emerge in general by consensus, not by voting ${ }^{2}$. This 'soft diplomacy' has resulted that both the International Health Regulations and the FCTC, the two legally binding the wHO agreements, do not include dispute settlements. Its language promotes and urges active cooperation between states and the wHO, without possibilities for external enforcement of public health measures. (World Health Organization 2005)

Furthermore, the wHO predecessors have been Regional Sanitary Offices, and this regional structure has been maintained when the wHO was founded in 1946. The six regional offices of the wHO have their own governance structures (regional committees). Coordination and coherence between the wHO headquarters and the regional offices have been a matter of concern, with fundraising and allocation not always connected to global strategic objectives. A case can be made for strong regional and country offices if only to be able to provide context-specific support. Needs in the African region are obviously very different from needs in other regions

2 It is noted that sometimes consensus not emerges between states, as in the case of the Intergovernmental Working Group on Public Health, Innovation and Intellectual Property in 2008. 
and priorities of work will differ. The current regional structure, with its different levels of management and performance, can be considered a key impediment to its effectiveness. (DeCoster 2013)

\section{Deliberation (epistemic reliability): civil society interaction with the WHO}

The relation between the wHO, civil society organizations' (CSOs') and other nonstate actors is controversial. In the wHO constitution, cooperation with non-state organizations and individuals is spelled out in several articles (art. 2, 18, 71). (World Health Organization 1946) Over the first decades, non-governmental organizations (NGOs) cooperated with the wHO mainly in the execution of programs and via professional consultations. Current principles governing the relations between the WHO and NGOs were agreed upon in 1987. (World Health Organization 2002)

In the 1990s, the wave of reform aspirations within the UN system prodded the recognition that solutions to overcome development, poverty and human rights issues could not be addressed by Member States alone. 'The universalization of Western liberal democracy as the final form of human government'-as described in Francis Fukuyama's The End of History (Fukuyama 1989)-became the contagiously dominant vocation. Firstly, it led the UN to look for a more active donor-driven embrace with the private sector as the new paradigm for development. The result is the 'critical platform' of the UN Global compact. Former director Brundtland was instrumental in re-engineering the way of working of the wHO along the lines of the Global Compact. The new global business model of multistakeholder cooperation she strongly pushed, kicked off the mushrooming of public-private partnerships in health, vertically directed at controlling a few diseases such as HIV/AIDS, tuberculosis and malaria. (Clift 2013)

Secondly, Brundtland tried hard to put health on the world stage and secure a role for wHO in the definition of the new development agenda underpinned by the values of equity, human dignity, and human rights. A key component of this vision was the achievement of the FCTC, in which NGOs have played an unprecedented role in their collaboration with the wHO Secretariat against the aggressive strategies of the tobacco industry. The process allowed NGOs to gain importance in the diplomatic policy deliberations on global health issues, with a very strong and competent monitoring role that continues today. (Lencucha et al. 2010)

In 1997, a meeting between the WHO and 130 NGOS delivered promising recommendations aimed at strengthening the collaboration between NGOS and the WHO at local and national levels. Considering NGOS as vital allies, the wHO's Health for All strategy even suggested that the wHO should appoint a formal NGO 
representative to the EB and the WHA. (World Health Organization 1997) The wHO did consecutively establish a partnership department, which developed the Civil Society Initiative (CSI). A new policy proposal for the interaction between the wHO and NGOS, building on the work of the CSI, was tabled in 2004 at the 57th wHA. (World Health Organization 2004b) The painstaking process was put on halt by a bare handful of countries (including China). Consideration of a new civil society policy has been halted since then.

\section{Financing: the core of the wHO reform}

The wHO, through its Director-General Margaret Chan, initiated a new process of reform in 2010. The need for predictability and sustainability of financing is at the core of the organization's reform initiative. (World Health Organization 2010) 75\% of the wHO's programs in 2010-2011 were funded through extra-budgetary voluntary contributions, $91 \%$ of which were earmarked for specific donor-driven priorities and programs. Uncontrolled donor dependence has directed the organization towards vertical programs for disease control relying mostly on drug donations. Funding for health systems has been systematically ignored. $18 \%$ of the donor funding comes from private foundations: the Bill and Melinda Gates Foundation (BMGF) is the second biggest funder of the wHO after the United States ${ }^{3}$. The vast majority of the BMGF's funding is channeled to the roll-out of existing vaccines and the research and development of new vaccines in low-and middle-income countries, mostly developed and produced by large pharmaceutical companies in the United States and Europe. (Harmer 2012) A new mechanism in the form of a financing dialogue was proposed to the EB in January $2013^{4}$. The financing dialogue is marketed as an innovative and transparent approach to secure the required funds. (World Health Organization 2012b) csos have expressed a shared concern that this approach may further institutionalize the wHO's donor dependence.

\section{The wHO's governance with external partners}

There is general agreement within the wHO Secretariat and with several governments that NGOs do bring a moral and qualitative strength to global health negotiations. Their role does help to promote more transparency and accountability in the different health negotiations, with healthier decision-making as a result. (World Health Organization 2002)

\footnotetext{
3 The BMGF donated US $\$ 446$ million in the period 2010/2011.

4 i.e. a venue where Member States and non-state donors come together after approval of the 12th General Program of Work and associated 2-years budget (a combination of Assessed contributions and Voluntary Contributions).
} 
Within the framework of the reform process, in 2011 the wHO Secretariat, taking the input of Brazil, suggested to convene a World Health Forum (WHF) as 'to increase engagement and trust in the international health system'. The WHF was to have the 'role of identifying from the different perspectives of its participants, future priorities in global health'. (World Health Organization 201la) Reassurance was given that the proposed mechanism would not 'usurp the decision-making prerogatives of the wHO's own governance'. Although NGOs broadly support inclusive consultation mechanisms on global health issues, their reaction against the WHF came as a surprise to many Member States, and to the Secretariat. Their strongest criticism was the notion of setting up institutional practices at the wHO for increasing participation of not-for-profit as well as commercial actors, while a robust mechanism to address conflict of interest lacking. (Medico International 2011 par. 5 \& 6) In the end, the wHF proposal was dropped by Member States.

Since 2011 several attempts have been carried out by NGOs to improve the negotiating dynamic for the wHO reform and raise key issues to give the reform a constitutional sense of direction. (World Health Organization 2012e) The Democratizing Global Health Coalition, a group of public interested oriented NGOS, stresses to regulate the wHO's engagement with external stakeholders, including NGOs itself. It advocates for clear regulations to be set in place to protect the wHO from undue private sector influence through the development of comprehensive conflicts of interest policy. (Medicus Mundi 2012) Until now, such a policy has not yet been seriously addressed by the reform initiative.

\section{The wHO and governance for global health}

The major responsibility for the future of the wHO lies with its Member States. While much lip service is paid to the need for capitalizing more effectively on the wHO's leadership position in global health, up to now the reform process has determinedly avoided a serious discussion on the WHO's role in global health governance. (World Health Organization 2010 par. 84) The related documents at the $132^{\text {nd }}$ and $133^{\text {rd }}$ EB were left aside without any discussion. (World Health Organization 2013b; e) In the wake of the global alert concerning NCDS, the wHO is expected to take a more active role in regulating key issues bearing an impact on health, including alcoholic beverages, food safety, and nutrition. The agency has tried already to address a number of challenges related to its role in a globalized economy. The trade and health agenda is a well-known case in point, with its controversial intellectual property chapter. The same can be said about the social determinants of health, climate change, and human rights. (Missoni 2011) It has been argued that a fundamental review and strengthening of the global governance system for health is required to address $21^{\text {st }}$ century health challenges. There is a distinct lack of overall leadership among 
all global institutions affecting health. This review and restructuring has to take place outside existing structures, in this case, the WHO's EB and WHA, in a purposespecific forum akin to the Brettonwood conference from 1944 that established the key multilateral institutions, but with far greater transparency and inclusiveness. (Labonté and Schrecker 2009) Others argue that the wHO's constitution needs to be revised, which 'could be used to fill gaps in global governance, hopefully in ways far more revolutionary than the meek evolutionary changes to the agency currently being discussed as part of the wHO's reform'. (Hoffman and Røttingen 2013)

\section{Democratizing the wHO: ways forward}

Regarding globalization, there is a dispute in political science between realists, complex multilateralists and cosmopolitan democrats. Realists argue that national political power and associated international agreements will continue for the foreseeable future, while complex multilateralists suggest that global social movements do already influence international organizations and bypass the national policy-making process but that national policies are also important. Cosmopolitan democrats view the world moving towards a new situation within which supra-national forms of accountable globalgovernance are being constructed. (Deacon 2003) Seen the historic supra-national developments within the EU, this form of 'regionalization' might also happen in other parts of the world. (Koenig-Archibugi 2010) Contemporary multilateral institutions such as the wHO should begin to reconstruct their legitimacy on a $21^{\text {st }}$ century basis, with more emphasis on democratic principles and less on national sovereignty. The right approach for the wHO is likely one of complex multilateralism, as in the contemporary world global democracy is unfeasible, but it would be wrong to close off the possibility of a democratic governance mechanism eventually developing on a global level. (Keohane 2006)

What does this imply for the WHO? In the wHO reform process, output legitimacy has already been addressed in the form of stronger internal governance procedures, improvements in management and organizational efficiency, and a results-oriented 12th general program of work, complemented by a bi-annual budget and outcome indicators for monitoring. (World Health Organization 2013d)

Concluding from the analysis on its democratic practices, there is still an absolute need to enhance the WHO's input legitimacy via its representation-, financing-and deliberation policies. As the wHO is a member state organization, discussion over its future role should be done at the country level. Diffusion of governance levels for health at the state level is becoming more complex. There is hence the need for inclusive and institutionalized cross-sectoral policy fora at the national level to shape the wHO policies (and wider global health issues). (Kickbusch and Gleicher 
2012) It will prepare and enhance the legitimacy of the country delegation to the wHO. Moreover, it will help to bring the WHO's role to the attention of the health community and public, and more into the political debate. Countries like Norway and Thailand already have such mechanisms. (National Health Commission Office of Thailand; Norwegian Ministry of Health and Care Services 2010) However, in current times many countries in Western Europe are privatizing their health services and downsizing their public sector in the wake of the financial crises and related austerity measures. Recent developments indicate a decrease of multi-bi financing for global health that could lead to less and not to more engagement by Member States in the wHO. (Sridhar et al. 2013) The debate on the wHO and global health can hence not be isolated from national public health programs that face serious budget cuts.

Regarding the WHO engagement with CSOs, there is a desire to re-initiate the policy developed under the Civil Society Initiative and proposed to the 57th wHA. (World Health Organization 2004b) This policy proposes clear principles for accreditation and collaboration with NGOs. Some non-state actors reflect mainly the interests of stakeholders from corporate entities and instrumentalize the WHO and its role in health as to fit with its own social responsibility image. A good example is the profile of the BMGF as the major philanthropist in global health, while at the same time the foundation is the major shareholder of Coca-Cola, a beverage whose contributions to health are doubtful. (Stuckler et al. 2011) Transparency and accountability are needed. The wHO cannot afford a blurred policy of collaboration with non-state actors; values, principles, inclusion and exclusion criteria that benefit public health outcomes have to be spelled out with conviction. The wHO could learn from the Food and Agricultural Organization, whose Strategy for Partnerships with cso could serve as an example, and which includes also community organizations and social movements. (Food and Agriculture Organization of the United Nations Council 2013)

The financing of the wHO remains the most salient point of the reform. The wHO does not necessarily require more funding in absolute terms-Its USD 4 billion bi-annual budget for 2014-2015 should be sufficient to fulfill its mandate-but it does require core funds and predictable sources for financing its key functions. Even though the proposed financing dialogue is expected to provide more flexible funding and transparency on voluntary contributions and budget allocations, it does appear a smokes-screen exercise as long as governments do not resolve the zero-growth policy of the agency. The dynamic of the wHO financing remains the same; only a small proportion of its funding is obligatory Assessed Contributions (AC) while the rest remains Voluntary Contributions (VC). The $132^{\text {nd }}$ EB has suggested that Member States explore how the proportion of AC can increase in the long term. (World Health Organization 2012c) Two possibilities to increase sustainable funding for the wHO can be seen. This is either via an agreed level of national revenues to be invested in 
global public goods and multilateral institutions. The wHO could hence be funded for its key role in global health protection and legislation. An alternative would be to develop international taxation for health, from which the wHO (and other health programs) can be financed. (Kickbusch 2013b)

From civil society, it is noted that engagement with some regional offices (such as the WHO-EURO and PAHO) is easier than with others. The wHO could do much more to democratize health at regional and country levels. Rather being mainly the technical referent and counterpart for ministries of health, the wHO could support and convene policy dialogue and democratic health fora. The Thai national health assembly or the 'Foro de Salud' in El Salvador, initiated by their respective governments, are good examples. (Foro Nacional de Salud de El Salvador) The WHO could capitalize on this and initiate policy fora in other contexts via its Country Cooperation Strategies.

Democratizing the WHO is about public trust that the organization and its members will value and consecutively act towards health for all. The political-economic determinants that either undermine or promote progress towards this vision must be made explicit, and it's the critical constructive role of civil society that will continue to do so. (Global Health Watch) 


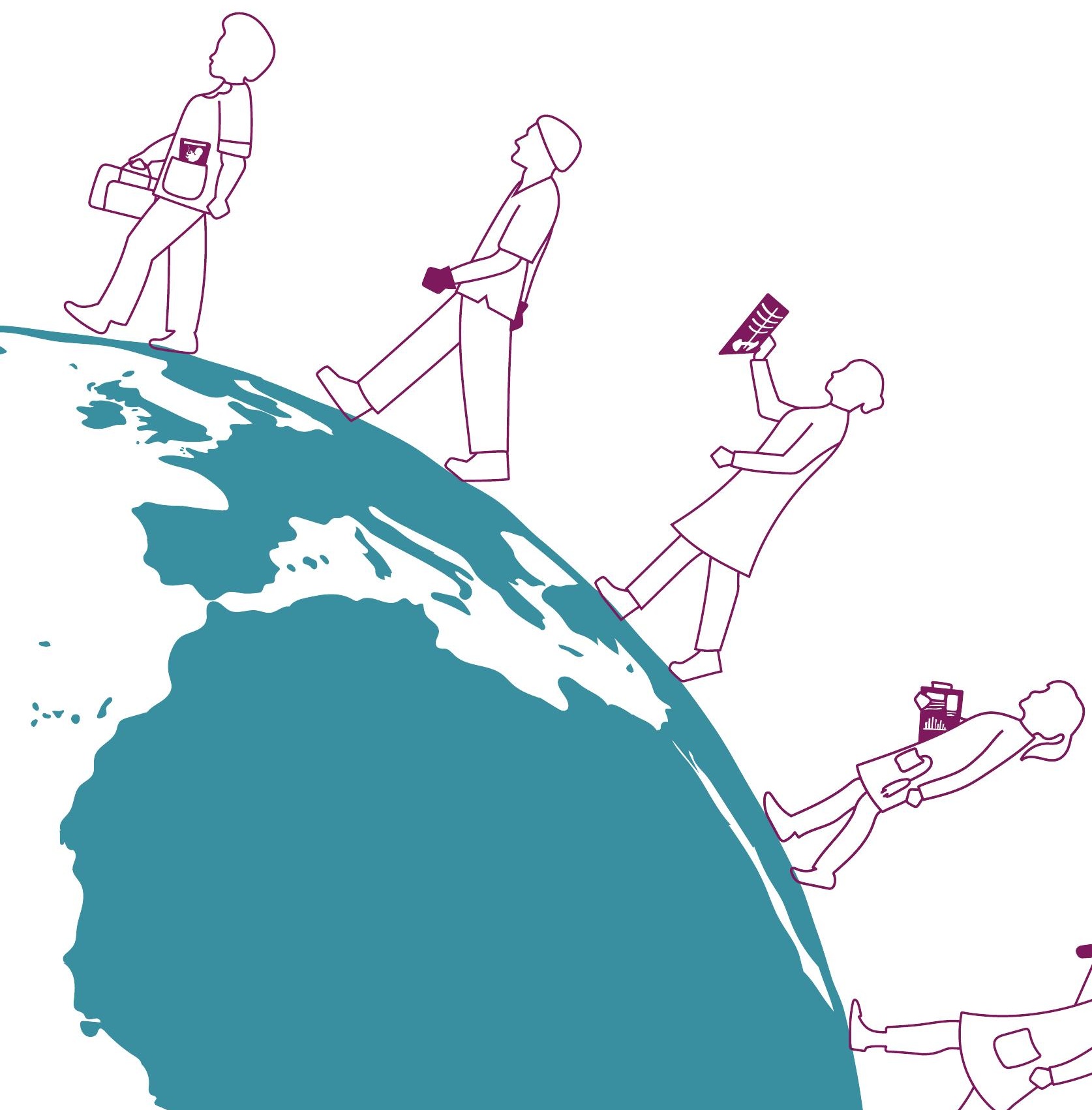




\section{The Code of Practice and its enduring relevance in Europe and Eastern and Southern Africa}

Van de Pas, R., Mans, L., de Ponte, G., \& Dambisya, Y. (2016).

The Code of Practice and its enduring relevance in Europe and Eastern and Southern Africa.

Human resources for health, 14(1), 30.55-63

doi:10.1186/s12960-016-0122-y 


\begin{abstract}
Background: The relevance and effectiveness of the wHO Global Code of Practice on the International Recruitment of Personnel will be reviewed by the World Health Assembly in 2015. The origins of the Code of Practice and the global health diplomacy process before and after its adoption are analyzed herein.

Methods and Results: Case studies from the European and eastern and southern African regions describe in detail successes and failures of the policy implementation of the Code. In Europe, the Code is effective and even more relevant than before, but might require some tweaking. In Eastern and Southern Africa, the code is relevant but far from efficient in mitigating the negative effects of health workforce migration.

Conclusions: Solutions to strengthen the Code include clarification of some of its definitions and articles, inclusion of a governance structure and a sustainable and binding financing system to reimburse countries for health workforce losses due to migration, and featuring of health worker migration on global policy agendas across a range of institutional policy domains.
\end{abstract}




\section{BACKGROUND}

\section{The origins of the Code of Practice}

The recruitment of health workers from abroad is part of an expansive pattern of skilled workforce migration that has existed since the rapid welfare state expansion of many countries in the 1950s and 1960s. Already in 1972, 6\% of the world's physicians were located outside their country of origin. (Bach 2003) The development of the World Health Organization (WHO) Global Code of Practice on the International Recruitment of Health Personnel (referred to as the 'Code' henceforth) was preceded by bilateral codes that aimed at mitigating the migration of health workers to richer countries. For instance, the United Kingdom Department of Health introduced a Code of Practice for international recruitment for National Health Service employers in 2001. (UK Department of Health 2001) A study that assessed the relevance of this code concluded that it was difficult to evaluate its actual impact due to a limited monitoring capacity, a multiplicity of factors besides active recruitment that influence the mobility of the workforce, and the limited visibility of this code in source countries. (Buchan et al. 2009) Other voluntary codes of practice and similar non-binding instruments have been widely criticized as weak and ineffective in mitigating workforce imbalances related to the migration of health workers. (Willetts and Martineau 2004) Despite this criticism, in 2004, the world health assembly mandated the Director-General to develop a non-binding code of practice on the international recruitment of health personnel. (World Health Organization 2004a) Simultaneously, the Joint Learning Initiative on Human Resources for Health and Development called for mobilizing and strengthening Human Resources for Health (HRH) as a key strategy to combat the health crises in the world's poorest countries and to build sustainable health systems everywhere. (Joint Learning Initiative 2004) In order to cope with the health workforce (HWF) crisis, the Joint Learning Initiative report proposed that effective country strategies should be reinforced internationally, "Ultimately, the crisis in human resources is a shared problem requiring shared responsibility for cooperative action". (Joint Learning Initiative 2004) This agenda was enforced with the release of the World Health Report 2006, Working Together for Health (World Health Organization 2006), and the creation of the Global Health Workforce Alliance (GHWA) in 2006. A decade of action on HRH thus commenced.

\section{A lost investment}

In an interconnected world, globalization and scarcity are closely linked. The fiscal realities that frame available public financing for health systems and HWF salaries are shaped by such issues as untaxed wealth, capital flight, wealth inequalities, etc. This 
fiscal crisis (including former 'ceilings' on expenditure of the HWF public wage bill, imposed by the International Monetary Fund in a number of African countries until 2007) has contributed to external migration, which, in turn, has caused significant savings in training costs to importing countries. (Global Health Watch 2014)

In nine African source countries, the estimated government-subsidized cost of a doctor's education ranges from USD 21,000 in Uganda to USD 58,700 in South Africa. The overall estimated loss of return on investment for all doctors currently working abroad is USD 2.17 billion, ranging from USD 2.16 million for Malawi to USD 1.41 billion for South Africa. The benefit to destination countries of recruiting trained doctors was largest for the United Kingdom (USD 2.7 billion) and the United States (USD 846 million). (Edward J Mills et al. 2011) As a counterargument Clemens reasons that many countries in the African region simply lack the absorption capacity to integrate the workforce either in the public or private sector. (Clemens 2011) Migrated African physicians in the United States and Canada send, on average, more than USD 4,500 per year to their countries of birth; these remittances will be used by private actors within the country and are higher than what leaves the public coffers. (Clemens 2011) However, several countries, especially those from the wHO African Region, when discussing the second draft of the Code during the wHO's Executive Board meeting in January 2009, expressed the view that it needed more 'teeth for enforcement and advised that it should include mechanisms to compensate developing countries for the migration to higher-income countries. (Taylor and Dhillon 2011) High-income countries, especially the United States, recommended not to link the provision of development assistance to recruiting practices. During the drafting and consultations on the Code that lasted from 2008 to 2010, the Health Worker Migration Initiative, a partnership of Realizing Rights (the ethical globalization initiative chaired by Mary Robinson), GHWA, and the WHO, facilitated the negotiations. This included the commissioning of a paper on potential strengths of non-binding instruments in international legal practice. The Health Worker Migration Initiative, together with Norway and the wHO Regional Office for Europe, also convened an inter-regional dialogue in Madrid in May 2010 to allow participants to get acquainted with the text and discuss content issues. The Code's non-binding character is considered as an advantage, as it allows flexibility, including with regards to future adaptation. The code sets forth a "deep legal and institutional framework" and may "promote deeper commitments" than legally-binding instruments. (Taylor and Dhillon 2011) A week later, the Code was adopted at the Sixty-third World Health Assembly, slightly modified though as high-income countries argued that the tone was too prescriptive or mandatory for a non-binding instrument -this modification has perhaps softened the sense of obligations amongst countries to comply with the different articles of the code. (Taylor and Dhillon 2011) The Code focuses on ethical international recruitment and fair treatment of migrant health workers, 
but also includes statements on self-sustainability in national HWF, international cooperation, support to developing countries, data gathering, and information exchange. Therefore, it comprehensively lays the ground for engagement on several aspects of the HWF, especially in developing countries.

The WHO recommended that the Code be incorporated into national policies and laws so that it can become legally binding. However, some states suggested that a more formal system for monitoring and implementing the Code was necessary for it to become a meaningful response to global HRH recruitment. The adoption of the Code, unfortunately, marked the end of a few 'good years for HRH' in global health policy. The economic crises in the United States and Europe led to a reduction of funds for GHWA and the WHO to effectively work on Code implementation and monitoring. Austerity in Europe and the United States put a strain on health systems, including the HWF. (Edward J Mills et al. 2011) Despite these resource constraints, a small but dedicated group of actors from different organizations and countries have been actively involved in the Code follow-up and implementation in recent years.

The HWF crisis should not be dealt with within its own thematic 'silo', but should rather be looked at in a systemic way. The global HWF gap has increased rather than decreased since the release of the World Health Report in 2006. Given current population growth rates in different regions in the world, an aging workforce, and an epidemiological transition to chronic disease worldwide, there is a desperate need for more skilled health workers. In 2013, approximately 7.2 million more midwives, nurses, and physicians were "missing and thus not in action"-and this shortfall is predicted to rise further to at least 12.9 million in the coming decade. (Global Health Workforce Alliance/wHO 2013b) The Ebola outbreak in West Africa in 2014 indicated how vulnerable health systems really are when a skilled workforce with core capacities for epidemic response is missing. The outbreak was yet another wake-up call for the international community and national governments to develop the global HWF urgently. (Sidibé and Campbell 2015)

\section{METHODS AND RESULTS}

Against this backdrop, the relevance and effectiveness of the Code has been assessed in a number of European countries and in Eastern and Southern Africa (ESA). These regions were selected for the present review given that the authors, active in academia and civil society, have been closely involved in Code follow-up and policy dialogue over the last few years. In this analysis, the authors provide their experiences with and insights into the uptake of the Code and its potential for future directions. 


\section{The relevance of the Code of Practice in Europe}

The period of implementation of the Code in the European region has coincided with the financial and economic crisis. The latter impacts directly on the relationship between investment in HWF development and HWF mobility, which is at the heart of the code: the resulting new intra-European Union (EU) wage imbalances and the persisting shortages of health workers confer to the Code a renewed relevance in the region.

Although countries in Europe have responded to the economic crisis in various ways, most have adopted large-scale cuts and public sector reforms: in the context of the austerity packages implemented in 2009-2011, public spending on health fell in many countries. (WHO Regional Office for Europe 2013) As health worker costs account for the largest share of spending on health, these costs have been a common target for budget cuts, also in countries where salaries are relatively low. (Thomson et al. 2014) Wage imbalances between countries (depending on changes in wages in immigration countries compared to emigration countries) or within countries (if the private and public sector have different rates of pay) are therefore changing considerably and have the potential to increase HWF mobility in the region and beyond. (Karanikolos et al. 2013) This new trend comes on top of already existing shortages: in 2012, the European Commission predicted in its Staff Working Document on an Action Plan for the EU Health Workforce, a potential shortfall of around 1 million healthcare workers by 2020, if no further measures were taken to meet existing challenges. (European Commission 2012b)

The response coming from EU institutions adopts a perspective that stems from considerations on the employment potential of the health sector.2 Health care is identified in the Action Plan for the EU HWF as a highly labor-intensive sector. (European Commission 2012b) As such, it is given a role in stimulating 'a jobrich recovery' from the economic crisis. Along the same lines, mobility of health personnel within the EU is facilitated, 3 as the assumption is that the EU Single Market functions as a mechanism to distribute health workers to where they are most needed. (European Commission 2012a)

Using this frame, public health considerations thus tend to take second place to market development approaches. The evidence shows, however, that the free movement of health workers leads to some seeking better opportunities abroad, creating a conflict in which personal and professional ethics sometimes collide (Tjadens et al. 2013) at the expense of an equitable distribution of health workers in the region and beyond. This is not entirely consistent with the principles of the EU's own Health Strategy and with the Health Programme 2014-2020, which assigns an important role to the reduction of health inequalities in the region. 
The Code can be a key tool to solve this incoherence, as it brings back a much needed public health perspective into the debate on the mobility of health workers by looking at the impact, in terms of brain drain, on health systems of origin. While the value of the Code as a policy framework to manage HWF mobility is formally acknowledged in several EU level policy documents (European Commission 2010), its voluntary nature implies that bold steps are yet to be taken to integrate its principles into the functioning of the Single Market: this can be done through a system of incentives and retention measures in countries of origin, and specifically by orienting EU Cohesion policy - which shapes the programming and deployment of Structural Funds - with a view to increasing support for the equitable internal distribution of a skilled HWF.

\section{Practices of Code implementation in Europe: the role of non-governmental actors}

In the above context, non-governmental actors, including health professionals' organizations, trade unions, nongovernmental organizations, and universities, are autonomously taking steps to implement the public health approach to HWF mobility promoted by the Code. Civil society organizations in eight European countries have been involved in documenting these efforts as a further indication of the relevance of the Code to actors on the ground. A selection of case studies, looking at both national and local levels, is briefly presented below. The case studies focus on key areas such as 'mobility, migration, recruitment', 'planning and forecasting', 'rights, working conditions, protection', and 'coherence, collaboration, solidarity'.

As the labor market becomes more globalized, rising demand is driving migration and mobility amongst health personnel.

- In the Netherlands, Wemos observed that hiring cheap personnel from other European countries or even from other continents is becoming an attractive option, both for home care provided via municipalities and for private (24-h) home-based care. Different civil society organizations and trade unions are seeking collaboration between recruitment agencies, Dutch inspectorates, the Ministry of Health, the Ministry of Social Affairs and Employment, municipalities, and other trade unions in order to ensure fair recruitment and the right of international health workers. (HealthWorkers4All)

Planning, forecasting, and providing for domestic HWF without resorting to international recruitment are key to the development of sustainable HWF globally and a fundamental step towards reducing brain drain. This also requires reliable data about inflow and outflow of health personnel. 
- In the United Kingdom, Health Poverty Action showed the engagement of the United Kingdom Royal College of Nursing in overcoming data limitations through the production of labor Market Review, which provides an annual picture of the United Kingdom nursing labor market, including the number of internationally recruited nurses and the wider global implications. (HealthWorkers4All)

- Redemptoris Missio documented how the National Chamber of Nurses and Midwives in Poland attempted to determine the actual scale of migration using direct requests to the appropriate authorities (mainly professional associations) in other European Member States. (HealthWorkers4All)

The Code extensively covers the promotion of (and respect for) fair labor practices as well as the provision of equal rights to all health personnel. Several case studies show that there are barriers, but also identify solutions.

- In Germany, Terre des Hommes analyzed the nurses' struggle for decent work at the Charité University Clinics in Berlin - a renewed trend to recruit nonEuropean candidates was observed, unfortunately occurring at the expense of improving conditions for the nurses already in the system. Thus, the recruitment of Asian or African nurses is the result of decreasing working conditions and may act as another 'push' for further cuts in wages and labor rights in the German nursing sector. (HealthWorkers4All)

- Terre des Hommes further analyzed the German-Philippine bilateral agreement for the recruitment of nurses, finding that the inclusion of social partners in both origin and destination countries at the right time, including in the monitoring of the agreement, allowed to shape a comprehensive agreement and avert detrimental consequences. (HealthWorkers4All)

- Another case study documented how increased collaboration between the European Federation of Public Service Unions, Verdi, and the Spanish Trade Unions for Health Workers (FES-CCOO and FSP-UGT) raised awareness that exploitative working conditions experienced by a group of Spanish nurses in Germany are unacceptable and that collective agreements must be respected. (HealthWorkers4All)

- In the Italian province of Florence, Amref documented how IPASVI, the professional federation of nurses, put in place the first Contact Point for international health workers: it supports and helps international colleagues find their way, addressing their concerns and concrete problems such as the recognition of professional qualifications, contract, and working conditions, as well as other general living and employment issues. (HealthWorkers4All) 
Contributions from Europe towards achieving a sustainable HWF and strengthening health systems worldwide require cooperation amongst several actors and a more common understanding and awareness - from global to local.

- In Belgium, the civil society-led platform for international health "Because Health" engaged key actors, including the Belgian Technical Cooperation, nongovernmental organizations, academic institutions, and private companies, on the issue of recruitment of foreign medical personnel, with the aim to harmonize, increase efficiency, and render more equitable the practices of Belgian development cooperation actors in this field. (HealthWorkers4All)

- Memisa's hospital twinning program stimulates professional development and exchanges between hospitals in Belgium and those in selected African countries. (HealthWorkers4All)

- Amref documented how a multi-stakeholder dialogue could effectively strengthen the role of the Italian National Professional Organization of medical doctors (FNOMCeO) in global health, based on principles of inclusiveness and solidarity. (HealthWorkers4All)

- Wemos demonstrated the role that health providers can take, through their Corporate Social Responsibility policies, in translating a global and European code at the local level in the Netherlands; this also needs various actors such as civil society organizations, trade unions, health care institutions, and recruitment agencies to help collectively raise awareness on this issue (HealthWorkers4All)].

- The center for Health Politics and services illustrated the case of Bulgarian specialist doctors being hired part-time in the neighboring Calarasi region of Romania, thus 'topping up' their Bulgarian salaries and in this way remaining in their region without having to migrate to another EU country. (HealthWorkers4All)

These case studies indicate that the public health approach to HWF mobility promoted by the Code is already translated into practice in many local and national contexts, thanks to the efforts by a variety of non-governmental actors. They are also a confirmation that the multi-stakeholder approach promoted by the Code is key to its successful implementation. These efforts, however, are often fragmented - it is time for a more systemic approach.

As a contribution towards this end, the civil society-led Call to action: A Health Worker for Everyone, Everywhere (HealthWorkers4All) was launched in 2014: it is currently gaining support at EU level, with more than 60 institutional endorsements 
indicating that there is a constituency of actors across Europe demanding Code implementation. The Call provides recommendations to EU institutions and Member States for strong HWF and sustainable health systems around the world.

\section{Code implementation in Eastern and Southern Africa}

A study in the ESA region, with 10 countries in the region represented, found that 3 years after the Code was adopted by the World Health Assembly, the main HRH concerns in the region were considered to be internal migration, maldistribution, and absolute shortages of health professionals, rather than external migration. (Dambisya et al. 2014) Regarding the content of the code, there was a perception among stakeholders that African policy interests in the negotiations on compensation and mutuality of benefits were not adequately covered in the final Code, and there were concerns regarding its voluntary nature. According to the research, Code implementation was lacking in all countries in the region, dissemination of the Code had is not materialized in the region, and only one country had a designated authority. Barriers to Code implementation included lack of champions/designated authorities, poor preparedness, weak mobilization of stakeholders, and low involvement of civil society.

The Code has not realized its potential to galvanize action on HRH in the ESA region, and yet it is one of the regions most affected by the HRH crisis. For instance, the topic of policy focus alluded to in the Code include improving migration monitoring(e.g., through a minimum core data set), managing migration flows(for instance, through bilateral agreements, memoranda of understanding, guidelines), HRH policy and practice (covering areas such as protection of the rights of migrants, promotion of circular migration, incentives for retention, better working conditions), strengthening health systems(through approaches such as HWF planning, education, retention strategies), and coordination, collaboration, and monitoring progress.

Clearly, most of the strategies needed to combat the HWF challenges in the region can be adequately addressed through implementation of the Code.

It goes without saying that the Code is relevant in driving forward the HRH agenda, and yet there has not been much progress in implementing the Code in the ESA region since it was adopted in 2010; most progress in implementation took place in European/Organisation for Economic Cooperation and Development countries. (Siyam et al. 2013) Challenges cited in the ESA region include lack of country champions, little effort by regional organizations and virtually no activity by civil society organizations (CSOs) in the region, the need to engage multiple stakeholders involved in the decision-making process on HWF migration 
and international recruitment, lack of coordinated and comprehensive data on health personnel mobility, weak national capacity to deal with HWF issues, lack of shared understanding between stakeholders, lack of inter-country cooperation in exchanging data, and lack of proper mechanisms for sharing good practices to better manage health worker mobility. (Dambisya et al. 2014) The silent voice of csos since the adoption of the Code is noteworthy. Civil society was part and parcel of the negotiations for the Code from the outset, throughout the entire process and up to the last minute when the Code was unanimously adopted at the World Health Assembly. The cSO voice has gone silent in recent years, however, partly because funding for further cso engagement on the Code has dried up. Without that voice, there is no one to whip countries and governments into action on the Code. A strong finding was that the Code content was not well known in the countries. (Dambisya et al. 2014) Strong cso action would have ensured proper dissemination and local interpretation of the Code.

The Code is relevant and has the potential to spur action on virtually all aspects of the HRH challenges in the developing world. Nevertheless, action has been lacking on both the part of governments and cisos.

\section{DISCUSSION}

The analyses of Code implementation in the European and ESA region indicate stark differences between these regions. In Europe, Code implementation and its underlying norms have been effectively addressed. Most countries are aware about the Code, and have a designated authority in place that monitors the different elements of the Code. In 2013, most of them also submitted timely reports to the World Health Assembly regarding the monitoring of Code implementation by its Member States. The wHO Regional Office for Europe has offered consistent policy advice and leadership to keep the Code relevant and under attention of its Member States. (Dussault and Buchan 2014) The EU Joint Action Health Workforce Planning and Forecasting, a 36-month project funded by the European Commission with the objective to provide a platform for collaboration and exchange between Member States to support them to prepare the future of the HWF, has concluded that "The principles of the Code are also relevant within the free movement zone of the EU". (World Health Organization 2013c) They suggest retention measures, circular migration, and better use of EU cohesion policies and the European Social Fund as policy options to mitigate unbalanced HWF mobility within the European Region.

In addition, a vibrant coalition of civil society (to a considerable extent also financed by the European Commission), academic institutions, professional associations, and 
labor unions ensures that the governance of HRH migration is addressed and remains on the policy agenda. The inter-sectoral approach with involvement of multiple actors as promoted in the Code is taking place in a number of European countries. Hence, the Code remains relevant for policy guidance within the EU. However, due to the financial crisis and related austerity measures, employment opportunities for the European HWF have diminished. There has been less recruitment from outside the European region, and more mobility of health workers between the European Member States. Migration mostly takes place from eastern and southern European countries to those in North and Western Europe. (EU Joint Action Health Workforce Planning and Forecasting 2015) This migration is mainly governed by European policies on the free market mobility of goods, services, and labor within the union. The European economic governance framework, the so-called 'European semester' provides guidance for the budgetary and fiscal space that the countries have commonly agreed upon. This economic framework also offers recommendations for reforming their health system, although this remains ultimately the responsibility and competency of the member state itself. The Code, in principle more tailored to addressing imbalances and ethical considerations considering health systems development between high-and low-and-middle-income countries, could also be used to mitigate this intra-European mobility, if slightly adjusted.

The ESA region offers a contrasting picture. The Code is still relevant in addressing HWF migration, but its implementation has been far from effective. Research has indicated that the number of African physicians in the United States workforce continues to increase substantially despite the adoption of the Code. (Tankwanchi et al. 2014) The absence of health workers in Sierra Leone and Liberia due to international migration was one of the key factors undermining an effective response by the health authorities to the Ebola epidemic. (Remco van de Pas and van Belle 2015) However, African countries have not been able to use the Code as a negotiating tool in health diplomacy to pursue their own policy interests as northern countries seem to prefer using development aid to address health worker issues rather than bilateral agreements. (Dambisya et al. 2014) There is a perception that these African interests are not taken seriously by the global health community, including most of the 'donor' countries in the North. Indeed, promises and pledges on funding for health systems strengthening have not been met over the last years. (Hill et al. 2011)

Additionally, weak political leadership, limited institutional capacity, and a silenced civil society have all played a role in failing to take the principles of the Code forward. However, poor dissemination and scarcity of resources might also explain, to a certain extent, why uptake of the Code has been hampered. In contrast to the EU, where there are several inter-governmental, research, and civil society projects funded in the field of HWF mobility, this is hardly the case in the ESA region. 
Additional resources could advance dissemination and advocacy amongst African policymakers to implement the Code's articles.

Perhaps there are simply more urgent issues to address than mitigating the migration of health workers. Further, in the short run, it might even be beneficial to have migrated health workers sending their remittances home so that their families can cover basic economic needs. The long term objective of building a national health system, often in settings where institutional governance arrangements are fragile, might not be the main priority for many ESA governments, hence the disinterest to implement, monitor, and report on the Code.

There are other issues that impede the effectiveness of the Code. "Active recruitment" (article 5.1) is not further explained, allowing space to interpretation and thus confusion as to what is considered "ethical" and what is not. (Tankwanchi et al. 2014) A second assessment is that the Code lacks an enabling governance structure supported by a sustainable financing mechanism for cost-sharing and reimbursing of resource-poor countries for the mobility and loss of their public workforce. During the negotiations on the Code, low-income and emerging market countries recognized that high-income states would simply not agree to more binding provisions on financial support to developing countries. As the Code is a living document, this situation is not carved in stone, however, and one could imagine a meaningful discourse on compensation in the future. (Taylor and Dhillon 2011)

A policy proposal has been made to recommend a global fee-supported system similar to that employed by UNITAID. This Global Health Resource Fund would basically use a dynamic fee structure that would oblige high-income countries and private sector actors engaged in the recruitment of resource-poor country health workers to contribute with funds earmarked for health systems strengthening and employment in the public sector. This fund would build upon the existing efforts of health systems funding platform by the wHO, the World Bank, the Global Fund, and the Gavi alliance. (Mackey and Liang 2013) This proposal matches well with current suggestions for an international health systems fund (L. O. Gostin and Friedman 2014) and there solution by the wHo's Executive Board Special Session on Ebola in January 2015 that called for "the establishment of a more extensive global, public health reserve workforce". (WHO Executive Board 2015)

Finally, the governance of HRH migration has become more complex over the years, as it is now at the nexus of wider global policy initiatives and debates. The "migration of health professionals is at the junction of the right to mobility, right to health and the right to decent work. It is about finding an acceptable compromise between the rights and obligations of migrant workers, employers, and governments based on sound research findings". (Yeates and 
Pillinger 2013) A key challenge is the coordination of responses within the different multilateral organizations that are involved in the multifaceted arena of $\mathrm{HRH}$ migration. It is for this reason that multilateral organizations share the view that the Code is unlikely to become a binding tool in the future. Nevertheless, one should explore broader public policy coordination affecting migration. This would include, amongst others, policy coherence with the International Labour Organisation's Multilateral Framework on Labour Migration. It is, in addition, necessary to make HRH migration an issue within the post-2015 development agenda, and in the debate on the role of global trade agreements in the quest for development. Global and regional trade agreements are likely to increase (temporary) labor migration. Therefore, there are many remaining questions about the global and shared responsibility for humans to have a universal right to access health services by skilled health workers. This leads to the following question: what role can a global alliance like GHWA play with respect to the monitoring of the Code, other codes, and global commitments to keep HRH migration on global policy agendas across a range of institutional policy domains? When the relevance and effectiveness of the code are discussed at the sixty-eighth World Health Assembly, and in relation to an upcoming Global HRH strategy, it seems vital to also discuss the necessary source and forms of global institutional leadership needed to refocus global attention on urgently needed HRH development and governance of health worker migration. (Yeates and Pillinger 2013)

\section{CONCLUSIONS}

When it comes to the relevance and effectiveness of the Code in the European and ESA regions, the picture is ambiguous. In a number of European countries, the Code is effectively implemented, partly due to a dynamic civil society engagement. The financial crisis, the related austerity agenda, and the internal European policy context have made the Code even more relevant within the EU in recent years. Conversely, in the ESA region, the Code remains very relevant due to the high attrition rate of health workers migrating abroad. The Code is, however, far from being effectively implemented, mainly because policymakers and civil society do not think the Code brings many benefits. Hence, it does not have a high priority for the governments and societies in the region. There are limited resources for dissemination, advocacy, and policy support to implement the Code. The nonbinding character and lack of compensation have led to a somewhat similar fate for the global Code as the bilateral and regional Codes of practice that were created over a decade ago. Solutions to overcome this situation would be to further clarify certain definitions within the Code and to develop a governance structure and a sustainable, binding financing system to reimburse countries for HWF losses due to migration. Likewise, there is 
a need to address the governance of HRH migration within the context of global international labor migration frameworks, the sustainable development agenda, and the development of global and regional free trade agreements. A human rightsbased approach, focusing on universal access to health care and health equity, should underpin such a global governance regime.

\section{ENDNOTES}

${ }^{1}$ Sixteen countries reported changes to health worker pay, almost all in direct response to the crisis (Austria, Cyprus, Denmark, Greece, Iceland, Ireland, Italy, Latvia, Lithuania, Montenegro, Portugal, Romania, Serbia, Slovenia, Spain, and United Kingdom). In some countries, especially those with economic adjustment programs, pay cuts have been substantial.

${ }^{2}$ The Action Plan for the EU Health Workforce is, in fact, an annex to the Commission's Communication towards a Job-Rich Recovery, which sets out a range of measures to encourage employment within the Europe 2020 framework for smart, sustainable, and inclusive growth.

${ }^{3}$ Free mobility of workers and services within the EU internal market is an economic imperative and a civil right enshrined in the Lisbon Treaty. The EU can increasingly be seen as a single labor market for health workers. It should also be seen as a protected market, given that Directive 2005/36/EC on Recognition of Professional Qualifications gives health workers from the European Economic Area (EEA) easier access to employment than their non-EEA counterparts.

${ }^{4}$ Health Workers for All and All for Health Workers is a partnership connecting civil society organizations in Belgium, Germany, Italy, the Netherlands, Poland, Romania, Spain, and the United Kingdom. 


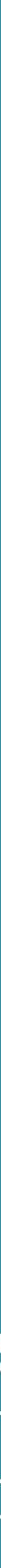




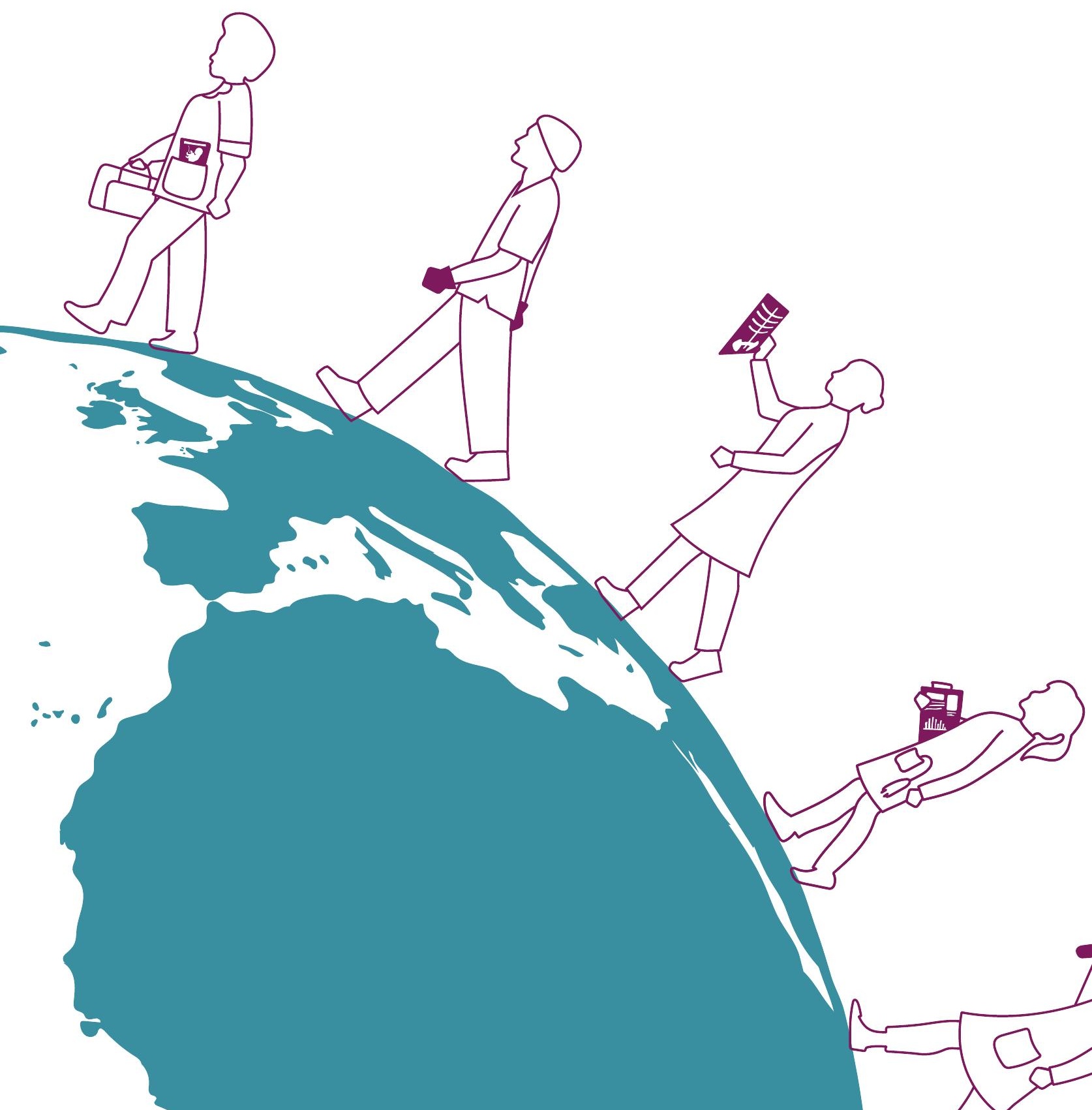




\section{1}

\section{The securitization of health and humanitarian space: Health workers under attack?}

van de Pas, R., Rushton, S., Krumeich, A., Townend, D. (2020).

The securitization of health and humanitarian space: Health workers under attack?

Medicine, Conflict and Survival. (under review) 


\begin{abstract}
:
Attacks against healthcare and humanitarian workers by adversaries have increased over the last years. Most of these occur in countries facing serious armed conflicts. This article deconstructs why attacks have increased and provides an interdisciplinary health and political sciences perspective. The main research question is whether a "securitization of health" discourse can explain these attacks against humanitarians. Secondly, it wants to assess whether there has been an erosion of humanitarian space for aid workers to provide care in a safe and neutral environment. This study aims to provide policy-makers and practitioners insights on overcoming attacks against aid workers. A review of the main theoretical concepts is provided as well as a classification of health workers' role in conflicts. The modern role of humanitarian NGOs under the banner of liberal humanitarianism is explored followed by three empirical cases on attacks against aid workers. Literature indicates that humanitarian space is a social construct, and a complex political, military and legal arena. The humanitarian system can act as a vector of Western values and interests that are not universally shared in the places where it intervenes. The analysis concludes that humanitarian workers have been complicit in undermining this space, even if unwittingly. The securitization of health provides challenges for aid workers that require a political dialogue and professional-ethical reflection on their legitimate role in conflict situations.
\end{abstract}




\section{BACKGROUND:}

The documentary film 'The new Barbarianism' released by the Centre for Strategic \& International Studies in 2018, tells the story that 'Healthcare and humanitarian workers are increasingly in the crosshairs as hospitals and aid centers have become part of the battlefield in today's wars'. (Morrisson 2018) It provides case studies of attacks against humanitarian healthcare in Syria, Afghanistan, and Yemen. It makes the point that the Geneva conventions are 'under siege' and tries to define recommendations for the international community to overcome the current gridlock of inaction in protecting humanitarian principles. (Morrisson 2018) Attacks on domestic health staff, as well as international health workers, have, at first sight, increased over the years. Most of these occur in countries facing serious armed conflicts, although not exclusively. The bulk of attacks occur in ongoing conflicts in Afghanistan, Somalia, South-Sudan, Syria, and Yemen. Globally, over the two-year period from January 2014 to December 2015, there were 594 reported attacks on health facilities and health care workers that resulted in 959 deaths and 1561 injuries in 19 countries with emergencies. Similar figures are seen for the years 2016-2018. (Fouad et al. 2017) The United Nations Security Council (UNSC), the main multilateral institution dealing with global security issues, has strongly condemned the attacks against medical facilities and health personnel in conflict situations. (United Nations Security Council 2016) Its resolution delivered a strong call to respect international humanitarian law which is based on the principles of impartiality, independence, and neutrality.

The international public health and medical humanitarian community, including the World Health Organization (WHO), has over the recent years regularly called for deepening the evidence base, categorizing types of, as well as understanding trends in health care services being under attack. This analysis is then normally coupled with a call to actors in a conflict situation to respect International Humanitarian Principles and the Geneva Conventions, which provides a standard of international law for humanitarian treatment during war. (Marie Paule Kieny et al. 2017; World Health Organization 2018f) The International Committee of the Red Cross (ICRC) has started a Health Care in Danger initiative aiming to increase public awareness, improving national responses to violence, and mobilizing a 'Community of Concern' addressing the issue of violence against patients, health workers, facilities and ensuring safe access to and delivery of health care in armed conflict and other emergencies. (ICRC 2017) Médecins Sans Frontières (MSF) runs the 'Not a Target' campaign calling for all parties to conflict stop attacks on healthcare workers, facilities and patients. (Medecins Sans Frontieres 2017) A structural analysis of the context, conditions and international relations driving this violence against health care workers is often missing in these projects. An interdisciplinary approach providing a health and political sciences perspective on this phenomenon might provide entry points to understand and prevent attacks on health care facilities and their personnel. 
This article tries to deconstruct why attacks on health care workers, working in contexts of conflict have increased during the last decade. The scope of this paper are domestic or international aid workers that work in humanitarian health programs in (post-) conflict-affected areas, either in government services or non-governmental organizations (NGOs), and that are funded through international cooperation. This approach follows a research need identified by a group of scholars and practitioners to 'Understand forms of and motivations for conflict-related violence towards health care' as part of a research agenda and foundation for protection of health services in times of violence. (Center for Public Health and Human Rights 2013)

To understand this trend of violence, it is also necessary to consider current policies in global health governance and the main paradigms guiding its directions. The 2014-2016 Ebola epidemic in Western-Africa and the ensuing debate on the governance gap in existing global health policies prompted Kickbusch and Reddy to suggest that 'global health is in the second phase of a political revolution'. (Kickbusch and Reddy 2015) Global health's political revolution is described as the phase that started 20 years ago due to a convergence of developments and trends. These include the changes created by the end of the Cold War, globalization, the increasing influence of non-state actors as well as a proliferation of serious health problems, including re-emerging infectious diseases, non-communicable diseases and changes in diets and lifestyles. All these together have led to an investment of vast political, economic and intellectual capital in global health over the last two decades. Global health is 'considered critical for national and international security, domestic and global well-being, and economic and social development in less developed countries'. (Kickbusch and Reddy 2015) Could it be that governments respond to a 'cosmopolitan moment' and strengthen transnational global health cooperation and humanitarian action to address increasing interdependence and structural global vulnerability, identified as a 'Global Risk Society'? (Beck 2012) Or might there be a backlash against this 'humanitarian' expression of globalization and do we witness a form of Global Health regression instead (Labonté and Gagnon 2010) as governments seem powerless to do anything about the deliberate destruction of health facilities?

This scoping paper examines aspects of this health-security nexus; a concept known as the Securitization of Health. A medical humanitarian focus is applied to describe the impact of this securitization ${ }^{1}$ and reflect on its relationship with humanitarian practice. The two sides of the health-security nexus are intertwined to the extent that

1 In this piece, we are using the term "securitization" as a label to contain a number of concepts relating to the safety, respect, and well-being health professionals, and beyond that, the sociocultural and medical significance of the work that they undertake in society. This does not relate to the use of "securitization" in financial contexts, where it means securing loan finance against defined assets. 
both security and medicine practices have changed which raises additional problems and challenges for medical personnel. This securitization of health trend potentially leads to a greater role for health and medical professionals in international affairs and security policy. (Elbe 2012) However, one doesn't only see a greater role for medics in security policy, but also a greater role for the military and other security actors in healthcare delivery. International health assistance has become considered by some governments a 'smart power' strategy, useful in stabilizing post-conflict settings or contributing to counter-insurgency operations, by winning the hearts and minds of populations and legitimizing the presence of government and its programs. There is a risk that these international health interventions can encourage violent attacks on health care providers by antagonists of such 'smart-power' approaches. (McInnes and Rushton 2014) It opens up the question of whether the medical "neutral and safe environment" (still) exists within a "humanitarian space" as is often assumed.

The main research question of the manuscript is whether securitization of health framework can provide an explanation for the increase of attacks on humanitarian health workers over recent years. Secondly, it wants to assess whether there is erosion of humanitarian space and whether humanitarian health workers have been complicit in undermining this space, even if unwittingly?

This paper aims to bridge the academic debate on humanitarian space and the policy and programmatic realities faced by humanitarian health workers. It calls on humanitarian policy-makers and practitioners to think about the political nature of their activities. It asks humanitarian workers whether, even if unwittingly, they been part of the undermining of humanitarian space and what could be possibilities to prevent this in the future?

\section{Structure}

The structure of this paper is built along the following lines. To begin with, an explanation and scoping review of the main theoretical concepts are provided. This includes issues such as the Securitization of Health, Humanitarian Space, Health as a Bridge to Peace, and a classification of health workers' role in violent conflicts and humanitarian settings. The paper will explore the modern role of NGOs and development cooperation for health under the banner of liberal humanitarianism and global health diplomacy. The current securitization of international health affairs is described as being part of 'smart power' and related "Development Diplomacy-Defence" strategies.

An empirical analysis of violence directed against health care workers during and after the Ebola outbreak in Western Africa; outlining several attacks targeting health 
care workers providing polio vaccinations; and a discussion on the role of the white helmets in the Syrian conflict are provided to supply insights into the securitization of health and polarization of humanitarian work.

In the discussion part, arguments are provided that nuance the current increase in violence to health care workers by providing a comparison to practices during the second world war and making the argument that humanitarian practice has always been the product of the dynamic and complex interplay of political, military and legal actors, interests and processes. What has changed over the last few decades is the role of humanitarian agencies and the space occupied during conflict. The discussion also touches upon the 'blurred' space between biomedical humanitarianism and the current global health security discourse, arguing that a co-governance and dependency approach is required to address current violence, whether in overt armed conflict situations or under the bio-political lens of increased cooperation on surveillance, surge response and containment to tackle infectious disease threats.

The paper ends with thoughts on advancing practice, policy, and research on the securitization of health and whether a medical humanitarian space can still be presumed in times of deep economic globalization, increased geopolitical tensions, and what is considered a global risk society.

\section{Health-security nexus}

Several developments over the last few decades indicate that health is treated increasingly as a security issue. A first observation is that globalization has brought about a paradigm shift dramatically increasing the frequency and speed of international travel and trade. The result is, among others, a pervasive feeling that pathogens can no longer be contained within state borders (if they ever could) and that a new approach for responding to disease outbreaks is required. The second common argument on the health-security linkage, which also became relevant during discussions on the reform of the global health security regime, is that pathogens might be weaponized, either by terrorists or through state-sponsored biological weapons programs. A third common argument is the claim that high burdens of disease can have social, political, economic, and military effects that threaten the stability of states and regions. (Davies et al. 2015)

The securitization of health might lead to a skewed priority setting in health. Highly virulent infectious diseases and bioterrorist threats drive international concern and investment in ways that do not necessarily reflect the burden of disease. This creates a disconnect between perceived threats and actual health needs at the national level. 
(James Smith 2015) 'In particular, the securitization of health leads to issues being seen either as existential threats requiring exceptional measures or as requiring technical/bureaucratic procedures that fall below the radar of democratic scrutiny'. (Nunes 2012)

The securitization of health is not the only way in which health and security are related in the health-security nexus. There is also what Elbe has called 'the medicalization of security'. Elbe has distinguished three dimensions of this concept. Firstly, whereas before insecurity was mainly thought of as a military or a political problem, insecurity is also increasingly being framed as a medical problem caused by the outbreak of disease, and which requires medical treatment. For instance, responses to the HIV/AIDS epidemic, more recently the Ebola epidemics, but also the fear of bioterrorism threats, would call upon images of destabilizing societies due to widespread disease. Secondly, this then leads to a greater role for medical professionals in international affairs as active actors practicing security. Finally, security problems defined in a medical sense call for interventions with a broad social and political reach. An example given is the way how governments stockpiled antiviral treatment during the H1N1 'Swine flu' pandemic in 2009-2010 preparing for mass treatment in case this was deemed necessary to protect the population. (Elbe 2012)

The question that a focus on the medicalization of security raises, then, is not only who are the real beneficiaries of a global health security intervention or what is the actual threat (Rushton 2011) but also who practices security including the role of health professionals as security actors. The exact fulfillment of this role might considerably impact on trust in and legitimacy of the medical profession. However, to understand this better, it is required to distinguish the different roles that health workers have before, during, and after armed conflict.

\section{The different dimensions and roles of health workers in armed conflict}

Buhmann and colleagues have identified four broad areas where health workers can and do play roles within situations of armed conflict, it being the 'military', 'humanitarian', 'development' and 'peace-through-health' domains. Health workers sometimes shift between these areas and roles so it is not a clear cut division. (Buhmann et al. 2010) In addition, the authors also identified four cross-cutting 'dimensions' which relate to the scope and focus of interventions; being 'insider/ outsider', 'individual/population health', 'policy and sector-wide intervention' and 'primary/secondary/tertiary prevention'. The dimensions, roles, and implications are summarized in the following table (Buhmann et al. 2010): 
Table 5.1: Overview of four roles in four dimensions of health work in violent conflicts.

\begin{tabular}{|c|c|c|c|c|}
\hline & Insider/outsider & $\begin{array}{l}\text { Primary, } \\
\text { secondary } \\
\text { or tertiary } \\
\text { prevention }\end{array}$ & $\begin{array}{l}\text { Individual/ } \\
\text { population } \\
\text { health }\end{array}$ & $\begin{array}{l}\text { Policy and } \\
\text { sector-change } \\
\text { dimension }\end{array}$ \\
\hline \multirow[t]{2}{*}{ Military } & $\begin{array}{l}\text { Insider: local/ } \\
\text { regional forces }\end{array}$ & $\begin{array}{l}\text { Not applicable for } \\
\text { fighting parties }\end{array}$ & $\begin{array}{l}\text { Individual, but } \\
\text { with population } \\
\text { approach to own } \\
\text { troops }\end{array}$ & No \\
\hline & $\begin{array}{l}\text { "Outsider: } \\
\text { international } \\
\text { forces" }\end{array}$ & $\begin{array}{l}\text { Secondary/tertiary } \\
\text { in peace-keeping } \\
\text { missions }\end{array}$ & & \\
\hline Humanitarian & $\begin{array}{l}\text { Outsider, } \\
\text { although often } \\
\text { employ locals }\end{array}$ & $\begin{array}{l}\text { Secondary, but } \\
\text { increasingly all }\end{array}$ & $\begin{array}{l}\text { Individual, but } \\
\text { increasingly both }\end{array}$ & $\begin{array}{l}\text { No, but } \\
\text { moving in that } \\
\text { direction }\end{array}$ \\
\hline Development & Both & $\begin{array}{l}\text { Tertiary, but } \\
\text { increasingly all }\end{array}$ & Both & Often \\
\hline $\begin{array}{l}\text { Peace-through- } \\
\text { health }\end{array}$ & Both & $\begin{array}{l}\text { All, with an } \\
\text { emphasis on } \\
\text { primary }\end{array}$ & $\begin{array}{l}\text { Population, but } \\
\text { sometimes both }\end{array}$ & Yes \\
\hline
\end{tabular}

Without going into detail on each dimension and role, the main consideration is that the areas where health care workers have roles in times of conflict have differences between them. Each of these areas may address the consequences of conflict on health (care) but may also have an impact on the conflict itself. It is important for health workers, managers, to reflect on their role in a health system, and how it may differ from others. The four roles by health care workers may differ in values and approaches over time and between conflicts. While there is complementarity, the role of a military-affiliated health personnel working on a health project initiated by armed troops might be very different from a civilian health worker working for a development NGO.

The notions of impartiality and neutrality are traditionally considered to be crucial to enable humanitarian actors to work amidst conflict, without becoming party to the conflict. Regardless, Humanitarian actors do become part of a violent conflict setting, and hence inevitably affect the conflict through the transfer of resources and implicit ethical messages; e.g. the attitudes and behavior of aid workers may send signals of superiority or even partiality. (Buhmann et al. 2010) Clear clarification of roles and responsibilities, also in communication vis-à-vis the population, might increase trust in the health workers. This is much required given the attacks on health care workers and health facilities in conflict areas, standing at 706 in 2018. (World Health Organization 2018f) 
An interesting, in-depth analysis of the history and roles of humanitarian actors is provided by Michael Barnett. Besides describing three different ages of humanitarianism since 1800 , he also provides the insight that humanitarianism has 'many mothers' and is known to idiosyncratic developments. It is crucial to understand that humanitarianism is characterized by the combination of forces of destruction, production, and compassion. Destruction, because patterns of war are shaped by the strategic ambitions of great and lesser powers. "If states believe that there is a convergence between their security interests and humanitarian actions; then aid organisations will find new opportunities; if otherwise they will confront significant barriers" (Barnett 2011, p. 22) The forces of production include capitalism and the global economy. A viewpoint is that capitalism is the structure and humanitarianism part of the superstructure that aids capitalism's reproduction and expansion. Marx identified already in the Communist Manifesto that economists, philanthropists, humanitarians, and organizers of charity operate to smooth over social grievances and help improve bourgeois society. "One could say that humanitarianism is a global welfare institution, and aid workers are social workers-appearing to be emancipatory, when operating as actors of social control." (Barnett 2011, p. 23) Humanitarianism is also a force of compassion and this trend can be traced back to the enlightenment with its discourse of humanity and that this related to the adherence to certain moral codes, one of them being that people should not suffer, including a feeling that mankind is causally and morally responsible for the misfortunes of others. (Barnett 2011, pp. 2529) Given this logic, humanitarian health workers are shaped by these destruction, production and compassion factors throughout their functioning. How these forces relate to one another in a particular conflict depends much on context. Selfawareness of their relationship to these co-existing forces might help aid workers to position themselves in areas of conflict and conduct their role in a constructive way. Given the focus of this article is securitization of health, it might be relevant in the next section to deconstruct its opposite paradigm: the peace-through-health angle and the role that health workers (could) take in this.

\section{Health as a Bridge for Peace}

The securitization of health has clearly diverged from the concept of Health as a Bridge for Peace (HBP), which has somewhat disappeared after having played a prominent role in the 1990s. The WHO integrated the HBP concept in its strategy on health for all in the twenty-first century. (World Health Organization 1998) Through projects in post-conflict states in the former Yugoslavia, e.g. by establishing intercommunity medical facilities so as to enable previously hostile groups to work together at the professional level, the wHO piloted this concept in the '90s. The appropriateness and effectiveness of the HPB approach have always been put in doubt, however. The concept was formally never abandoned but was not retained 
in the Millennium Development Goals (MDGs) peace-building objectives. There is a belief in some headquarters that health assistance programs are vital for humanitarian and development objectives and should not be diverted by broader political concerns. For some, the peace-building objectives bring the risk for health professionals to be involved in political conflicts that they would rather avoid. (Rushton and McInnes 2006)

In 2012 an initiative was taken by the network Medical Peace Work (MPW) to revive the Health and Peace concept. It explored questions like whether it is a universal concept and applicable in all phases of conflicts? Based on analysis from MPW programs in Southern-Thailand, Myanmar, Syria and Northern Iraq it concluded that in all the four areas 'peace' possessed a highly charged political connection. For health workers whether or not to engage in peace work very much depended on the context. In all contexts, the primary concern was security for the families of health workers as well as patients. Of equal concern was coping with the potential impact of military operations on health services. Health and peace might be universal concepts but their application in the field is certainly not given the different political contexts and sensitivity. Documenting existing activities of national health professionals in their efforts preventing and mitigating impact of violent conflict is needed. (Chan Boegli and Arcadu 2017)

Arya asked the question whether there is a divide between the Global North and South in relation to peace and health work. Most victims of war are in the Global South, while countries in the North often precipitate or help fuel violent conflicts. Thus, the predominance of lived experience of conflict is in the South, but the discourse, discipline and conceptual elements are largely coming from the North. An ultimate aim of global health is to seek justice, reach across divides, and respectfully share knowledge and experience, recognizing inequality of circumstance. For this it is needed, sometimes, to acknowledge the (historical) roles of health professionals as perpetrators of violence and injustice or as beneficiaries of colonialism. Such recognition is the basis for any reconciliation and resilience. (Arya 2017)

\section{Humanitarian Space}

Given this complex, political and even dangerous, space of peace and health work it is hence not surprising that humanitarian aid workers feel more compelled to function in a constructed, humanitarian space. Humanitarians traditionally base their work on four core principles that, they argue, enable them to follow their values and not the interests of others. These principles are humanity, impartiality, neutrality, and independence. Like weak states defending their sovereignty, aid agencies cling to the principle of a 'humanitarian space', as this space and its 
principles allow agencies to be innocent by association. Humanitarian's perceived apolitical character is part confidence trick and part self-delusion but it helps aid agencies to do the impossible. Nevertheless, humanitarians do practice politics. It's a particular brand of politics, operating in Foucault's famous aphorism that 'the misfortunes of men must never become the silent left-over from politics'. (Barnett 2011, p. 38) Humanitarians practice a politics of resistance, of humanity, of protest against an international sacrificial order that sacrifices so many in the name of justice, of life. Emergency agencies work to maintain the appearance of being apolitical because it helps them practice their kind of politics. Humanitarian organizations have been tirelessly lobbying, pleading, cajoling and shaming states to respond to tragedies around the world and opt for more progressive foreign policies. Advocacy is politics by another name. (Barnett 2011, pp. 37-41)

Although much of the discourse on the erosion of humanitarian space implicitly harks back a supposed 'golden age', the concept of humanitarian space is actually relatively new. The ICRC only formally adopted the' Fundamental Principles of the Red Cross' in 1965, amongst which the most important were impartiality, neutrality, and independence. (Barnett 2011, p. 137) The term 'humanitarian space' appears to originate in the Cold War conflicts in Central-America, and became more widely used after 1990. The concept means different things to different people and remains poorly defined and understood. Collinson and Elhawary, who reviewed the concept, clarify that humanitarian space can be understood (1) as agency space; (2) as affected community space; (3) as International Humanitarian Law; and (4) as a complex political, military and legal arena. 'Humanitarian space is therefore an unavoidably wide and subjective concept'. (Collinson and Elhawary 2012) Actors like MSF and ICRC used to frame the concept as an apolitical agency space, claiming a shrinking of humanitarian space over the last decade as well as a perceived "politicization of humanitarian aid'. Nevertheless, the story of humanitarian action during the cold war period as well as during the conflicts in Somalia, Rwanda, and former Yugoslavia indicates that humanitarian space demands to be understood in essentially political terms. During the cold war period, international humanitarian response was highly restricted as politics demanded respect for stave sovereignty. NGOs and the ICRC, not yet representing a major force, were often refused admission by parties in a conflict. During the early '90s, there was a relatively brief period of expanded political space for neutral and impartial action by NGOs. With direct financial and political support from Western governments, United Nations (UN) agencies and NGOs built a moral and media-appeal to alleviate human suffering, especially in settings with weak authorities in Sub-Saharan Africa. This, in conjunction with broader changes in international relations, contributed to what could be seen as an erosion of the assumption of non-interference in sovereign states. The inability to prevent the Rwanda genocide and its aftermath led to an increased acknowledgment of the 
huge human costs of failing to intervene in some circumstances, and a growing realization that there needed to be greater coherence between humanitarian NGOs, political and military actors to attain sustainable peace and stability. NGOs accepted cooperation with expanding UN peacekeeping forces in several conflict settings as well as with the North Atlantic Treaty Organization (NATO) in the case of the Kosovo war. In areas that fell outside of Western strategic interest humanitarian action was still essentially a substitute for robust foreign policy. (Collinson and Elhawary 2012)

The very concept of humanitarian space has thus not been fixed and has arguably never firmly existed as a separate impartial and neutral space outside or above state politics. It must be understood as a construct; a product of power and practice, shaped by the politics of that time, notably the decline of the Soviet-Union. NGOs and UN-agencies scaled-up their humanitarian programs rapidly and were considered legitimate actors to intervene in complex and violent conflicts. However, their space and agency was, and is, mostly shaped by the Great Power interests. 'The petty sovereignty of NGOs was governmentalized' (Collinson and Elhawary 2012) as we can see in a further transformation of humanitarian practice in the new millennium.

\section{Liberal Humanitarianism, Global Health Diplomacy, and Smart Power}

Before the turn of the millennium, there had been a rapid increase and expansion of humanitarian and medical NGOs. This coincided with a near-hegemonic dominance of liberal, democratic, cooperative development models in international health. Official funding for humanitarian assistance increased from USD 2.1 billion at the beginning of the '90s to USD 16.7 billion in 2010, including private donations. (Collinson and Elhawary 2012)

Many of these NGOS provide services in conflict and post-conflict settings that are otherwise under-governed, fragile and thus a possible threat to peace and stability. The designation of the HIV/AIDS epidemic as a threat to international peace and stability in a 2000 UNSC resolution has also been noteworthy. (United Nations Security Council 2000) 'Fighting' the HIV epidemic was included as a separate goal in the MDGs and the creation of the Global Fund to Fight Against AIDS, Tuberculosis and Malaria (Global Fund) triggered investments in the provision of HIV prevention and treatment programs by NGOs in severely affected countries, many of them in Sub-Saharan Africa.

This facilitated a subtle form of soft diplomacy by Western states that aimed to counteract the violence and conflict following from an increased trade in weapons, military interventions and race for scarce raw commodities in so-called 'fragile states'. While the welfare state in European countries is built on a social contract 
between citizens and state, there is no such equivalent beyond the state. Global policy agendas such as the Millennium and Sustainable Development Goals as well as the financing and fast growth of NGO practice provide an alternative, far from perfect, liberal humanitarian attempt to achieve some loose approximation of a globalized version of a social contract. Global Health Governance and Global Health Diplomacy (GHD) implying the multi-level and multi-actor negotiation processes that shape and manage the global policy environment for health are essential in securing accountability, transparency, and deliberation required to advance such global health goals. (Kickbusch 2011)

The events of September 11, 2001 (but not uniquely) brought development and humanitarian action back into the security sphere. Development became more closely aligned with Diplomacy and Defence objectives, hence one talks now of the '3Ds' of foreign policy and security. The 3Ds focus on state-building, stability, and good governance, which are of course based on political values and political ideas on the role of the state and other agencies. (Sondorp and Bornemisza 2011) Civilian health and medical personnel that are working with NGOs in the context of highly politicized '3D' operations might be less aware of the potential ethical dilemmas this entails. Sondorp and Bournemisza noted that:

"Overall, health professionals working in conflict-affected and fragile states may increasingly be confronted with dilemmas emanating from tensions between their primary motivation to improve health in the most equitable way and the stabilisation and state building goals of their sponsors. For instance, in the current climate, a health professional working to address the obvious health needs of the Afghan population will almost inevitably be part of efforts to legitimize a possibly illegitimate regime." (Sondorp and Bornemisza 2011)

McInnes and Rushton have described how, in the case of health interventions in Afghanistan and Iraq, health became part of a wider strategy based on 'smart power'. The latter is being defined as a combination of smart and hard power to reach desirable policy outcomes. Health became part of an expansive military counterinsurgency strategy. There is hence not only an expansion of humanitarian NGO's and GHD efforts but likewise a militarization of health that has crept into the domain of public health interventions during times of conflict. This has led to medical ethical tensions as 'health for health's sake' programs and broader geopolitical/strategic programs collide. The authors urge caution in utilizing health assistance for strategic ends as it politicizes the health sector and reduces a humanitarian space. Moreover, the strategic benefits of smart power and health interventions might actually be limited. (McInnes and Rushton 2014) In addition, there is anecdotal evidence that such smart power approaches are part of the explanation for why there has been an increased attack on humanitarian aid workers. 
The three examples in the following section indicate the political nature of humanitarian practice in the health and relief domain. The examples on the attacks against health workers in Guinea during the Ebola outbreak, violence directed towards polio immunization staff as well as the contestations concerning the "White Helmets' in Syria have all in common that health and humanitarian practice cannot be de-linked from the socio-political context in which it interacts. These empirical cases provide a reflection on the drivers behinds attack against health care workers and how they could have been prevented.

\section{The Ebola Epidemic in West Africa and the politics of fear}

The civil-military response to the Ebola outbreak in West Africa did not only blur the lines between a health and a humanitarian crisis but also between civilian, military and security actors. This became most evident when MSF called for military intervention on 2 September 2014, as they deemed this the only possibility (and last resort) to restore order and prevent violent conflict. Actors involved in the Ebola outbreak response argued that this was regarded by the international community as providing legitimacy to the subsequent deployment of military forces. (KamradtScott et al. 2015) During and after the Ebola epidemic, MSF has portrayed itself as the prime medical-humanitarian professional association active in international affairs. Its international president Joanne Liu participated in several high-level political panels and committees, including at the UNSC, to set up and reform global health security regimes such as the International Health Regulations and UN mechanisms to address humanitarian crises. (United Nations Security Council 2016) In Guinea, the organization faced distrust and resistance while working in local communities as people perceived the organization to be biased and favoring the Guinean government regime in power. MSF has been accused of 'monopolizing' the Ebola outbreak and undermining cooperation with local health authorities. (Remco van de Pas 2015) Eight health workers, not from MSF but including staff from the Federation of the Red Cross and a Guinean journalist, were killed in the Guinean forest region more precisely the village of Womey. They were there providing Ebola prevention and health activities. This region was characterized by decades of perceived discrimination and social exclusion by successive regimes. It is still unclear exactly what precipitated the violence in Womey but the military invaded and looted the town shortly after the killings sending thousands fleeing their homes. Health interventions, benign in their intentions, must be understood as also be rooted in a longer local history of how such interventions have been interpreted by communities. (Benton 2017) Nunes, while reflecting on MSF's role in the Ebola epidemic, argues that given the legitimacy of the organization MSF workers could assume a political role that seeks not merely the immediate alleviation of suffering but also the redressing of longstanding vulnerabilities. He also argues that the politics 
of fear that pervades the global health agenda focused on crisis management and disease containment, should be countered by a politics of solidarity based on a global responsibility toward the health of others. In times of globalization, a more activist conception of borderless is required, one that recognizes the persistence of borders pertaining not simply to geography but also to the re-inscription of harm, vulnerability and based on differences of gender, race, class and sexual orientation... MSF has the responsibility to help bring down the walls of global health'. (Nunes 2017) Here, it is basically argued that humanitarian space is a complex political arena in which the organization has to position itself politically.

\section{Polio vaccination and attacks on health care workers}

Attacks on aid workers providing polio immunization have captured international attention over recent years. Most of these attacks have been in countries where the virus still leads to morbidity and, perhaps not surprisingly, are prone to an armed conflict. Most of the Polio cases, as well as violence directed towards Polio vaccinators, have taken place in 4 countries: Nigeria, Pakistan, Afghanistan and Syria. Although resistance to Polio vaccination by Islamist insurgents can be traced back to 2003, this resistance has become increasingly hostile in the last couple of years. Observers stress a relationship with the Central Intelligence Agency's (CIA's) use of a fake Hepatitis immunization program to collect DNA from Osama bin Laden's family members before his assassination in 2011. Attacks could be then considered the indirect impact of undermined trust and the suspicion that immunization campaigns are a cover for espionage activities. Moreover, the use of drone strikes in northwest Pakistan is said to have amplified enmity to polio vaccination campaigns because the insurgents suspect that Polio workers were carrying out surveillance in order to identify targets for drone strikes. (Kennedy et al. 2015) The role of a Pakistani medical doctor in, probably unconsciously, tracing Bin Laden's family via this immunization program has been described in detail. (Mullaney and Hassan 2015) His story is a cautionary tale about the consequences that can spiral out of control when health professionals get too close to intelligence operations. While the humanitarian and public health community would argue that it is armed conflict in general that is driving the persistence and re-emergence of polio, it seems to be, in several areas, specific insurgency groups that undermined the immunization campaign and are attacking polio workers. This increasing hostility is ascribed as being a reaction to the counterinsurgency strategies of domestic governments and international actors. As an indirect consequence, Polio campaigns got interrupted and wild Polio Virus cases re-emerged in Afghanistan and Pakistan, with a recorded 22 cases in 2017. (Global Polio Eradication Initiative 2018) 


\section{Syria's White Helmets}

The weaponization of health care and targeting of facilities in Syria over the last years led the UNSC to condemn attacks on health workers and facilities in conflict situations in resolution 2286 in 2016. (United Nations Security Council 2016) In the Syrian conflict, there has been an evolution and expansion of the role of health workers and their organizations. The Union of Medical Care and Relief Organizations, with international support, was treating 50,000 patients per month in 2015 in nongovernment controlled areas. (Fouad et al. 2017) Another crucial institution is the Syria Civil Defence, known as the White Helmets, a volunteer group of 2900 rescue workers that has attracted a lot of international attention due to its role in emergency responses to armed attacks on civilians. Defined as an unarmed and neutral humanitarian force, and with professionals from a variety of backgrounds, they serve populations in non-government controlled areas. They receive funding and support from American and European governments to conduct their work which includes not only rescue operations and medical evacuations but also the provision of basic public services such as reconnecting electrical cables. Over 250 White Helmets have reportedly been killed while saving others. (The White Helmets 2018) What is striking in the weaponization of care and its humanitarian response in Syria, is the role of social media, images and how journalists report on aid and health care in the armed conflict including attacks on health care workers. This dimension deserves careful analysis and research in itself but the polarization in the (social) media, including the presence of very active twitter bots, on the mandate and agency of the White Helmets is considerable. While the White Helmets are lauded in some places as humanitarian heroes, including via a Netflix-streamed documentary (Von Einsiedel 2016), in others, such as a Russian backed online propaganda campaign, they are considered to be an Al-Qaida linked terrorist organization. (Giraldi 2018) The White Helmets have become the target in a true modern information war. This is seemingly not only because of their rescue work but also because of the documentation that they provide via the use of cameras producing footage that has helped organizations like Amnesty International to verify the aftermath of airstrikes. In August 2018 about 100 White Helmets and their families were evacuated from Southern Syria to Jordan with the support of Israeli defense forces. This was done on request of the United States (US) and a number of European countries. This has been considered a humanitarian rescue mission as their lives were under immediate threat. The United Nations High Commissioner for Refugees (UNHCR) is involved in a resettlement scheme for the aid workers to be taken up by a number of Western countries. (Wintour 2018) This evacuation is likewise part of an information war with the White Helmets being accused of 'being part and parcel of the attempt to overthrow a legitimate government and install a regime friendly to Western, American and Israeli interests'. (Giraldi 2018) While it is difficult to disentangle facts from fiction it 
indicates that health and humanitarian workers have become weaponized, willingly or unwillingly, in the instability and insecurity seen in Syria. Consecutively they are considered to be legitimate targets by parties in the conflict.

\section{Discussion: Empire of Humanity?}

The preceding sections and cases have deconstructed several consequences of the health-security nexus and what this implies for the role and safety of health personnel, especially in humanitarian settings. Likewise, there might be a parallel convergence whereby health services in certain settings have become militarized. The final section of this paper provides a reflective analysis of this securitization trend, the transformation of humanitarian space and what it implies for policy and practice to protect health personnel.

Barnett concludes his historical study with the suggestion that humanitarianism could be compared to a form of Empire, a term mainly attributed to nation states and their imperial expansion. Humanitarianism shares the following characteristics with empires: first, they involve long-distance rule by one people over the other; second, they lack legitimacy because they rule without the blessing or the participation of the people; third, power radiates downward and for the purpose of advancing the empire's interests. (Barnett 2011, pp. 220-24) Also, a crucial difference with empires is noted. While empires fight for their immortality, humanitarianism is dedicated to its own destruction, at least in theory. Although humanitarians have always been sensitive to the power of states they have often been amazingly insensitive to the power they have over those they want to help, mainly in the form of paternalism. (Barnett 2011, pp. 220-24)

Arguably international aid workers seek their paternalistic legitimacy to practice and act in the form of universal values referring to the notion of a 'shared humanity'. But, of course, these shared values differ according to the local socio-cultural context, the historical connotation, grievances as well as perceived interests of these international actors. More and more the appeals to expert knowledge as well as measurable outcomes have given the field an aura of being evidence-based and rational in the way it functions. One can think, for instance, of the Sphere project, that has aimed to set humanitarian standards. (Sphere Association 2018 ) All this moral and expert authority can obscure the very presence of real power. The acceptance that humanitarian intervention based on a shared humanity also might valorize military force to protect people is known as the Responsibility to Protect Principle. This has been seen in Afghanistan, during the Ebola outbreak in WestAfrica, and in a more distant past in Kosovo and Rwanda, and is part of the answer why humanitarian health workers working in the health-security nexus are part and 
parcel of complex political relations. Humanitarian personnel can become part of a political, even violent, conflict.

For several years, the wHO has, together with international partners, systematically collected data on attacks against health care workers. These figures indicate that the trend of attacks against health care workers has not decreased since the start of data collection in 2014. (World Health Organization 2018f) Most of the attacks are taking place in about 15 countries plagued by violent conflict and fragility. A comparative analysis by Physicians for Human Rights on violence against health care in 6 conflicts during the last 30 years came to the conclusion that a proportionally high number of attacks have taken place in Syria, indicating a general trend that has worsened during this period. (Briody et al. 2018) It is difficult to predict how this conflict, and those in other countries, will develop in the near future but the problem of attacks against health and aid workers is likely to remain over the coming years. The nuance needs to be made that an increase in attacks on aid workers must also take account of the fact that many more agencies and aid workers are trying to operate in dangerous places compared to the past. Before the end of the cold war, humanitarian actors often didn't have access to conflict locations while nowadays humanitarians routinely operate in dangerous environments at the center of conflicts. (Collinson and Elhawary 2012)

Harman and Wenham while reflecting on the Ebola response in 2014-2015 argue that in the humanitarian-health nexus, described as two 'separate regimes of Global Health', it is imperative that there is a co-governance and dependency between global health and humanitarian actors. They are critical about the current direction by the wHO to programmatically putting together health emergencies (Zika, Yellow Fever) and emergencies with health concerns (such as the conflicts in South Sudan or Yemen). This would provide further confusion to the governance of infectious diseases and fails to address the divide between global health and humanitarian actors. (Harman and Wenham 2018) A case in point is the development of the Emergency Medical Teams (EMT) unit within the wHO that has as its mission: 'To reduce the loss of lives and prevent disability in sudden-onset disasters, outbreaks, and other emergencies through rapid deployment and coordination of quality-assured EMTs' (World Health Organization 2018 ) The EMT, by setting up minimum standards and principles for internationally deployable teams, provides a critical role in contributing to national, regional and global response capacities. Normally this is done during natural disasters and health emergencies but given the 'blurring' between state, civil and military actors in health emergencies these teams might be dragged into situations of violent and protracted conflicts hence providing a certain security risk for health personnel. (Kamradt-Scott et al. 2016) An in-depth analysis of the role of EMTs during the ongoing Ebola outbreak (2018-2019) in violence-ridden Eastern 
Democratic Republic of the Congo (DRC) might provide guidance on how to further the EMT initiative in a safe and sustainable manner. (World Health Organization 2018h) Unfortunately, there have been already several attacks on Ebola treatment centers managed by MSF. Observers have noted that there is especially distrust towards health outreach workers when they are accompanied by security forces. (Nguyen 2019) In April 2019, an epidemiologist deployed by the WHO in the response to the Ebola outbreak in DRC was killed in an attack on Butembo University Hospital. (World Health Organization 2019f)

This leads then to a reflection on whether a 'neutral and impartial' humanitarian space can still exist for health aid providers to conduct their work? Barnett suggests that while discourses of humanity imply non-discrimination, discrimination might actually be a natural order of things, even necessary to realize our humanity. To an ordinary human being, love means nothing if it does not mean loving some people more than others. Humans are part of concentric circles of multiple communities, providing them with a sense of belonging and identity. This also affects who humanitarian agencies feel obligated to provide services to, and discriminate via existing attachments, selective population groups. (Barnett 2011, p. 231)

For instance, humanitarian NGO's do apply the 'Do No Harm' approach in which not providing aid to those in need is ethically defensible through the human rights discourse. This emerged after reflections on the use of humanitarians to support a genocidal regime in the refugee camps of Goma in 1996. But the application of the 'Do No Harm' principle is perceived by some scholars and practitioners as 'tantamount to playing god'. (Chandler 2001) The 'new humanitarian' approach is selective, politically and culturally motivated. Such a tendency of blaming the 'undeserving victims' has led to support for sanctions and the refusal of aid, e.g., in the '90s to Serbia and nowadays to Iran. (Chandler 2001) There is an inability to generate strict impartiality as some form of discrimination is required to generate a sustained sense of humanity. (Barnett 2011, p. 231) Humanitarian health workers hence also discriminate, perhaps unconsciously, in their services. Much of this type of health work involves crossing boundaries and injecting values that are presumed to improve wellbeing. 'There are many reasons why local populations might reject those who come bearing gifts (and provide services: note authors) but one is surely the fear that humanitarians are not content to truck, dump and run but instead seek permanent revolution'. (Barnett 2011, p. 231)

This is coherent with the understanding of humanitarian space as a complex political, military, and legal arena. The humanitarian system is frequently exclusive, dominant, internally competitive, and fragmented. It can also act as a vector of Western values and interests that are not universally shared in the places where it intervenes. The ultimate responsibility for ensuring respect for humanitarian principles does not lie 
with humanitarian organizations but rather with political authorities and military forces. It requires humanitarians to come to agreed positions and actions that can more effectively influence these actors. Furthermore, political and military actors might still oppose principled humanitarian action and principles if they feel that they can benefit from a more politicized humanitarian response. Attacks are often designed to demonstrate 'the might of the attacker, the weakness of the victim, the inability of the opposing force to prevent such an attack'. (Collinson and Elhawary 2012)

A study on the failure to protect humanitarian workers during the Second World War provides valuable lessons for current policy and practice. First, advances in military technology and air warfare made it difficult to create safe spaces for civilians and humanitarian workers. Second, the close association of humanitarian workers with national military efforts may have made them targets of attack. (Verma 2017) Drone and missile technology and urban warfare used in current conflicts threaten the principle of distinction between military and civilian targets and make it harder to find a secure space. Humanitarianism is still often closely tied to military efforts. This became very clear in Colin Powell's statements when he called aid agencies 'force multipliers' and 'an important part of our combat team'. (McInnes and Rushton 2014) The ongoing blurring of principles and political objectives by United Nations missions has an impact on the security for humanitarian workers. A sharpening of the distinction between humanitarian and military activities is required. (Rushton 2011) However, the analysis in this paper indicates that the role of health workers in conflicts and emergencies is intrinsically influenced by several, sometimes contrasting values, norms, and contexts. It requires reflection by humanitarian organizations on how to best effectuate the values that fit their professional role in services and how this relates to structural factors that shape their deployment and agency.

A structural transformation is that current global governance, including in global health and humanitarian action, is considerably influenced by the perception of a global risk society, which is a society that is increasingly occupied with debating, preventing and managing risks that it itself has produced, such as climate change, antimicrobial resistance or terrorism. (Beck 2006b) There is a key distinction here between risk and catastrophe. Risk does not mean dealing with catastrophe. Risk means the anticipation of catastrophe. There is also a fundamental irony of risk: science, the state, and the military are becoming part of the problem they are supposed to solve! (Beck 2006b) This risk anticipation and its management enables securitization of health insofar that it reinforces the governmentality of statehood by linking public health activities such as infectious disease control, management of biological threats with foreign policy and security activities. For instance, the 'pathologization' of societies (helpless, traumatized, victims) may have served to legitimize external interference by humanitarian or other actors. This entails the 
bypassing of democratic decision-making and public scrutiny indirectly leading to the encroachment of management and government in the lives of individuals and societies. This is not intrinsically 'good' or 'bad' but it asks for a cautious and reflective stance when understanding the connection between health, security and politics at both the domestic and international levels, including actors like health professionals and their functioning in health systems during or outside emergencies. (Nunes 2012) Global health risks, potentials for crisis and related vulnerabilities are not evenly distributed. The Ebola outbreak indicated that these health risks follow structural injustices, neglect and render invisible the people most affected by such global risks. (Nunes 2016) Likewise, most victims of war are still in the Global South while countries in the North and other Great Powers often help fuel violent conflicts. (Arya 2017)

Risks are also not evenly distributed in humanitarian organizations. International NGO's demonstrate a considerable mismatch between the security resources, support, and capacities provided for international and national staff. It is noteworthy that the rate of incidents affecting national staff averaged two or three times the rate of accidents affecting international staff between 1997 and 2008. (Collinson and Elhawary 2012) There remain tension and disagreement on who benefits from the global health security and humanitarian regime as well as over the distribution of its costs. Deepening deliberation and fair global governance mechanisms on the role of humanitarian and health staff in these programs and policies could potentially increase the legitimization concerning 'securitized' health strategies. At least it could enable a global reduction in the attacks against health care workers.

\section{CONGLUSION}

Considerable analysis, modesty, and reflection are needed by humanitarian aid organizations and health professionals on their agency, power, and position in old and new arenas of conflict. This has become a transnational challenge beyond geographic borders making it a true 'cosmopolitisation community of global risks' requiring different localized contextual approaches and solutions. (Beck 2011)

The neutrality, non-discrimination, and impartialityoften assumed byhumanitarians and medical professionals are constructed political concepts. International NGO's have tried more recently to reclaim apolitical humanitarian agency space so as to distinguish themselves from contested 'Smart Power' and '3D' interventions that have politicized aid. However, the humanitarian space has always been a complex political and legal arena. Whether such a space provides a safe working environment for humanitarian health personnel requires a permanent assessment and dialogue 
with local and international authorities, given the contextual situation these humanitarian NGOs find themselves in. The emergence of health professionals in international policy debates and coordinated activities, on enhancing security and stability (e.g. the humanitarian missions of 'rescuing' refugees in the Mediterranean Sea come here to mind) may also pose a risk for the aid and health workers involved. Organizations and individuals have to reflect on their political and ethical positions in these actions and whether they want to partake in them or seek possibilities for action in a different way. Humanitarian workers, even if unwittingly and not as a general rule, might have been through their actions contributing to undermining health aid and international cooperation. To put it in Gramscian terms: humanitarian staff may be regarded as contributing to, or maintaining, a hegemonic discourse unconsciously legitimizing security policies by states that exclude and discriminate a group of 'others'. Counter-hegemonic actions, whether its social activism, terrorism or vandalism, might find humanitarian workers being an 'easy' target as they are the perceived frontline representatives of an unjust state. This trend fits with a broader political analysis that, in general, there is a recession or even erosion in the democratic functioning of states and that the interests of their citizens are no longer safeguarded. (Diamond 2015) The unrest and violence it triggers is also played out in the humanitarian and health care arena.

As a final note, there is need for caution, self-reflection, and deliberation as a basis for overcoming the attacks on aid workers. Rather than merely calling on the parties in a conflict to respect the neutrality and impartiality of health personnel, Alex De Waal advises the following:

"Militarizing public health is a strategic error. Security and public health experts know this and have tried to steer global health and security policies in a direction that is informed by the best evidence and analysis...

.... We also need to maintain social services and establish confidence in public health measures that may be undignified, alien, impoverishing, and unpopular. These demand the types of skills and relationships that only local health providers possess, combined with public education, community consultation, and a clear commitment to human rights". (De Waal 2014) 


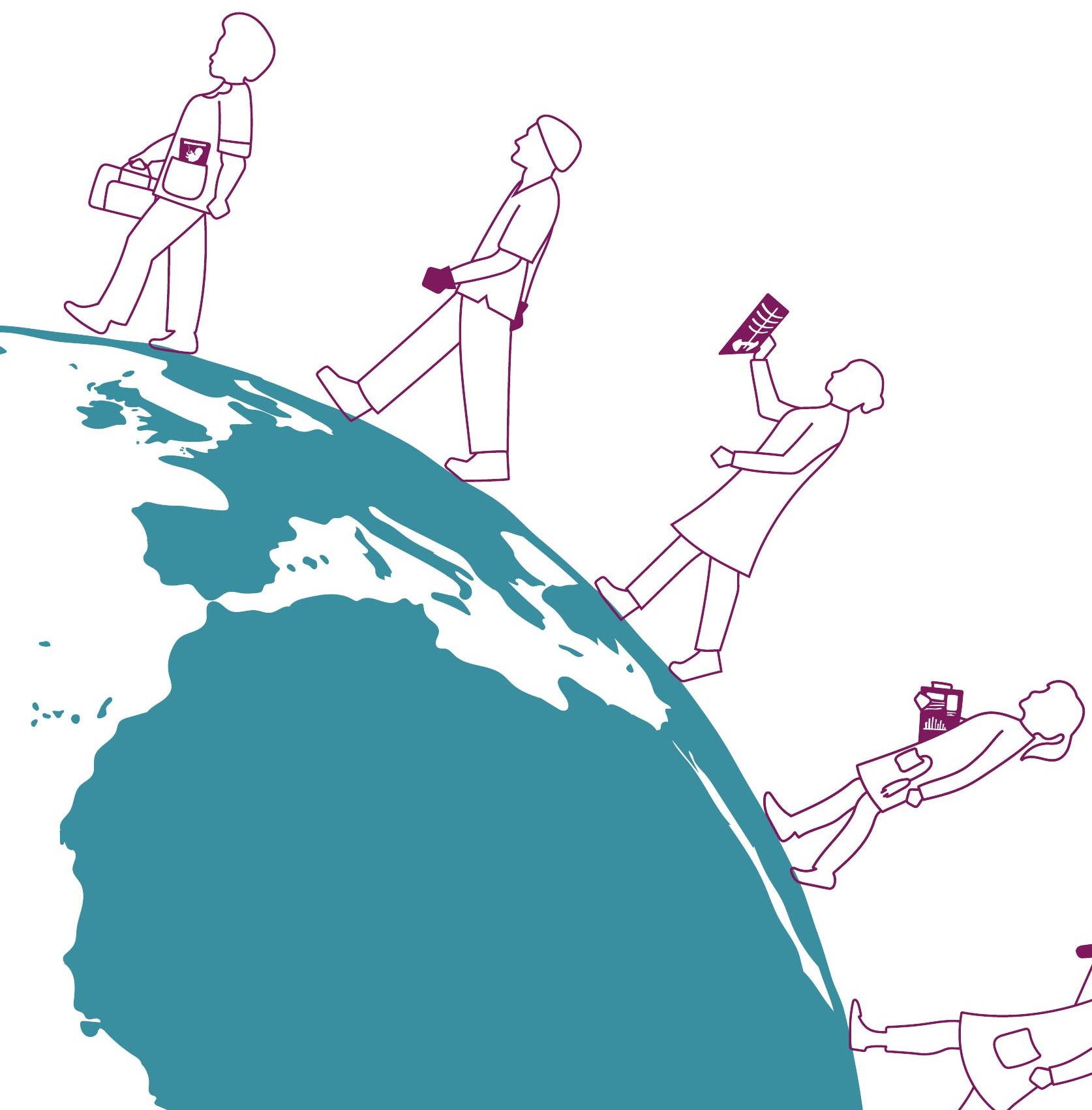




\section{2}

\section{Ebola, \\ the epidemic that should never have happened}

van de Pas, R., \& Van Belle, S. (2015).

Ebola, the epidemic that should never have happened.

Global Affairs, 1(1), 95-100.

doi:10.1080/23340460.2015.989724

This isn't a natural disaster. This is the terrorism of poverty.

(Paul Farmer) 
At the time of writing (November 2014), the death toll from the Ebola outbreak in West Africa has exceeded 5,000 people. This number is likely to be an underestimate. The first Ebola outbreak occurred four decades ago, in 1976, in former Zaire, current Democratic Republic of the Congo (DRC). (Piot 2012) There have been over 20 outbreaks since, but the current one has led to more cases and fatalities than the aggregated total of all the former outbreaks. Why is that the case? In this article, we argue that besides the challenge of ecological thresholds and other driving factors, an analysis of the political economy underlying bilateral development cooperation for health might provide insight into the dynamics of the current epidemic. Secondly, an assessment of the response of the World Health Organization (WHO), the mandated United Nations (UN) institution to deal with international outbreaks of epidemics, may shed light on what the actual policy space is and point to future challenges related to managing emerging infectious diseases such as Ebola, through international cooperation. (Chang 2005) The social, cultural and ecological determinants that contributed to the current Ebola outbreak, the marginal position of these West-African countries in the global political economy, as well as the belated international response indicate a complex interplay between different systems and (political) drivers. This requires interdisciplinary research, necessarily complementing public health with other perspectives. Land reforms, deforestation, and investment in palm oil plantations have lowered the ecological threshold for Ebola to emerge. (Wallace et al. 2014) In addition, rapid urbanization on the African continent, with inadequate housing, water, and sanitation in slums, provides fertile ground for human to human transmission on a large scale.

This is the first time that Ebola has emerged in the West-African region. After an initial outbreak in a rural forested part of Guinea in December 2013, a small, localized outbreak ensued, without proper diagnosis of the virus nor containment until mid-March 2014. By the time national authorities and the international humanitarian organization Médecins sans Frontières realized that this was evolving into a serious epidemic, the Ebola virus had already spilled over the borders to Sierra Leone and Liberia via dense intra-country trade networks and intra-regional human mobility. In April 2014, the virus popped up in the dense urban environments of the capitals Conakry (Guinea), Freetown (Sierra Leone) and Monrovia (Liberia). Never before has an Ebola outbreak taken place in urban populations. Tragically, dilapidated infrastructure, lack of equipment and qualified personnel mean that the health services in these countries are a source of transmission. Poorly protected health workers carry a heavy brunt, as in such conditions, they are easily infected while taking care of seriously ill (or dying) Ebola patients. By 7 November 2014, 549 doctors and nurses had been infected with Ebola, and 315 had died. ${ }^{1}$ 
Even before the current crisis inflicted this deadly toll on health workers, the three countries were already severely underresourced. Liberia had just 57 doctors and 978 nurses and midwives in 2008, while Sierra Leone had 136 doctors and 1017 nurses, far below the WHO recommended staffing levels. Out of fear of becoming infected, the population avoids health centers or hospitals in all affected countries. Utilization rates have dropped considerably. In Guinea, for example, hospital visits dropped by $54 \%$, antenatal care by $59 \%$ and vaccination rates decreased by $30 \% .{ }^{2}$ As a consequence most of Ebola's victims may well be dying from other causes at the moment; women in childbirth, children from diarrhea and so on. (Farmer 2014) Although reports of the epidemic spiraling out of control became more frequent, and the need for coordinated international action increasingly evident, it took the wHO until 8 August to declare the Ebola crisis a public health emergency of international concern (PHEIC). ${ }^{3}$ Many have criticized the WHO for its slow response. Confronted with several simultaneous public health crises (among which the Middle-Eastern Respiratory Syndrome and the H7N9 flu), the WHO appeared to be overstretched. Furthermore, it seems the wHO was focusing much attention on the eradication of polio in these crucial months - one of the main private donors of the organization, Bill Gates, is a passionate advocate of polio eradication. In January 2014 the United States (US) delegate to the WHO's Executive Board asked to declare polio a PHEIC. This was endorsed by the International Health Regulations Emergency Committee in May 2014. This was questionable, as only 413 cases of polio were reported worldwide in 2013, and the risk of the disease spreading globally is relatively low because of high levels of vaccination coverage in all but a few countries. ${ }^{4}$ A case can be made that an external private donor hence distorted the wHO's priority-setting process. At the same time, the Member States of the WHO underfunded the core task of communicable disease surveillance and response. (Hawkes and Arie 2014) This is not really a surprise, as the WHO currently finds itself embroiled in "the dynamics of open source anarchy", in which powerful private philanthropic foundations exert undue influence on the international political agenda setting of sovereign states. (Fidler 2007) Moreover, the wHO was hamstrung by its own members, who effectively restricted the WHO's autonomous (operational) capacity by tying their funds to specific programs. For the past decade, richer countries have decreased funding for the wHO's core functions and as a result, the wHO controls only $30 \%$ of its budget. (Van de Pas and Van Schaik 2014) We suggest they are committing a shortsighted, strategic error that might eventually come back with a vengeance in the form of a complex epidemic. Indeed, the diminution of the role of the WHO undermines the long-term interests of high-income countries including the European countries who usually preach the importance of effective multilateralism. (Mahbubani 2013; van Schaik and Battams 2014a) On 18 September 2014, the Security Council called the Ebola outbreak a "threat to international peace and security" and unanimously adopted Resolution 2177. The un General Assembly established the United Nations 
Mission for Ebola Emergency Response (UNMEER) following General Assembly Resolution 69/1. (United Nations General Assembly (UNGA) 2014) Its mandate is to coordinate the global Ebola response and manage international funding. An estimated USD 1 billion is required to contain the disease. By the end of October, over USD 100 million had been pledged to UNMEER, although actual spending so far is much lower. In practice, this means that many of the Ebola Treatment Centers, promised in September, are not functional yet. At this moment, only a quarter of beds needed to treat and isolate patients in the three countries are in place. (Hawkes and Arie 2014) The European Union (EU) countries and the US are the major funders and decision-makers of the World Bank (WB) and the International Monetary Fund (IMF), two institutions that pledged USD 530 million to help Guinea, Liberia and Sierra Leone. The EU and its Member States have committed more than $€ 600$ million in humanitarian and development aid to the affected countries since March 2014, and have recently pledged to increase that figure to $€ \mathrm{l}$ billion. ${ }^{5}$ Although the EU and its Member States have invested a considerable amount of development aid into the fragile health systems of these countries (mainly post-war Sierra Leone) over the last decade, there are incoherencies that require further explanation. For instance, over a recent two-year period, a little over 5\% (USD 3.9 million out of USD 60 million) of EU health sector support to Liberia was actually passed on by its ministry of finance (see "Cut in UK aid", 2014). The unpreparedness of the three countries to deal with a disease like Ebola, is to some extent a consequence of post-war fragility and unstable leadership (in the case of Sierra Leone and Liberia), but can also be explained in part by a decades-long lack of investment in core public health infrastructure and services. This can be directly traced back to sparse spending on public services, following the dictates of IMF loan conditions. Under the 'Structural Adjustment Programmes' (that the three countries have been subject to) (SAPRIN 2004), the "macro-economic stability" paradigm curtailed public spending. These restrictive fiscal regimes hindered the ability of least developed countries to invest in their health systems. In combination with the impact of political instability, this led to run-down facilities, inadequate numbers of health personnel and demoralizing working conditions. In Sierra Leone, per capita annual government expenditure on health is only USD 31, a fraction of the USD 443 spent by the South African government (see WHO, 2014). Especially worrisome is the wage bill ceiling the IMF sometimes sets for public services. (Rowden 2014) This ceiling contributes to the "push factors" that drive migration of nurses and doctors from these countries. It is estimated that $40 \%$ of Liberian doctors are currently working in the us alone. (Fuller Torrey 2014) The need for considerable investment in the public health capacity in least developed countries thus requires a revision of IMF Fiscal and Monetary policies as they display obvious shortcomings. (Rowden 2014) There are some signs of hope: Members of Parliament (MPs) of the United Kingdom (UK) Parliament's International Development Committee acknowledged in October that declining levels of 
international aid to health systems development (including from the UK) might have contributed to the current Ebola outbreak ("Cut in UK aid", 2014). After the world was shaken up in 2003 by the severe acute respiratory syndrome (SARS) epidemic, the WHO overturned a Westphalian-inspired system of country-led responses to transnational disease. The wHO's Global Outbreak Alert and Response Network (a global pool of expertise and technical institutions) helped to contain SARS within four months after its emergence. This experience facilitated agreement among Member States on the revised International Health Regulations. ${ }^{6}$ However, Ebola shows that the WHO (and its Member States) have forgotten some of the hard lessons of SARS. (Horton 2014) The 2007 World Health Report warned that at least " 57 countries, most of them in sub-Saharan Africa and South-East Asia, are struggling to provide even basic health security to their populations". (World Health Organization 2007) Despite promoting public health allegedly being its core task, the wHO's capacity in this respect has been severely curtailed. The organization has a budget that is the equivalent of a third of the US Centers for Disease control (CDC). In 2011, the wHO had to cut its budget by nearly USD 600 million, as funding could not be guaranteed. The organization's emergency response unit was downsized and epidemic control experts left. (L. O. Gostin and Friedman 2014) Although funding for global health cooperation quintupled over the last two decades, much of these funds bypassed the WHO, and were spent via bilateral cooperation, global publicprivate philanthropic foundations or the many international NGOS. In the meantime, the coordination, legislative and technical norm-setting functions of the wHO were largely ignored by the international community. (Van de Pas and Van Schaik 2014) In 2003 global health lawyer David Fidler claimed that SARS was "the first PostWestphalian pathogen". (Fidler 2003, p. 485) The Ebola virus can be characterized in similar terms. Despite the fact that the epidemic remained largely restricted to the three western African countries (at least till now), fear-bordering on hysteria, in the US for example-spread across the globe. (Horton 2014) Draconian measures such as refusing visas for western African travelers to Canada and Australia, as well as quarantine measures for West-African travelers to China, have been taken (see “Canada suspends visas”, 2014). (The Guardian 2014a) In other countries, such measures are being considered as well, often under pressure from public opinion. The evacuation of a number of international health staff infected with Ebola to their countries of origin, receiving high-quality care and experimental drugs, whereas their West-African counterparts are dying without proper care, moreover raises tricky ethical questions ("Why are western health workers", 2014). Ebola is the tip of an iceberg of (re)-emerging infectious diseases that prosper in a world where increased global mobility of humans, food and animals increases the risk of transmission, resulting in more and more transnational health threats. (Karesh et al. 2012) The Ebola outbreak illustrates that countries and regions do not yet know how to respond to ever-increasing globalization, even if it has been around for some 
time. (Stiglitz 2002) In 2015 we believe post-Westphalian public health should take center stage again. This horrific crisis should make humbled nation states realize that together we need to reinvest - urgently - in the wHO, the internationally mandated institution to defeat health threats for global health security. (Remco Van de Pas and Van Schaik 2014; van Schaik and Battams 2014a) Paradoxically, Ebola provides the opportunity to strengthen global health security, and one could even think of the creation of a the wHO-led fund for health emergency contingencies, or even an international health systems fund which would enable low income countries to boost capacities for local health systems, surveillance and early response mechanisms for emerging infectious disease outbreaks. (L. O. Gostin and Friedman 2014) The Ebola epidemic should never have happened. In other African countries, like Uganda (in the past) and DRC (now), Ebola outbreaks were rapidly contained. Actually, the virus is not highly contagious as it is not an airborne disease. The high case fatality rates are the signature of a virus that cannot spread widely - it kills its host too rapidly. ${ }^{7}$ In the current outbreak, a complex set of factors at different levels have come together to create the perfect storm. (Wolfe 2013) Ebola is a wake-up call and only through international cooperation and solidarity can this perfect storm be dealt with. This avoidable public health crisis should spark a (renewed) global commitment to coordinate and build up fragile health systems. Moreover, it can kickstart a much needed discussion about how, if we truly want to improve the health of a global citizenship, international cooperation should be motivated by cosmopolitan ethics and not solely driven by the health security interests of sovereign states. (Lencucha 2013) It also poses the question of how the EU and its Member States can do more to strengthen the wHO's surveillance role to diminish the risk of unnecessary spread of deadly infectious diseases. 


\section{NOTES}

1. See the wHO Ebola Response Map Situation Report, 12 November 2014. Retrieved from the wHO website: apps.who.int/iris/bitstream/10665/141468/1/ roadmapsitrep_12Nov2014_eng.pdf?ua=1

2. Data as presented by Guinean students of the Master in Public Health, Institute of Tropical Medicine, Antwerp, 4 November 2014.

3. See Statement on the 1st meeting of the IHR Emergency Committee on the 2014 Ebola outbreak in West Africa, 8 August 2014. Retrieved from the wHO website: www. who.int/mediacentre/news/statements/2014/ebola-20140808/en/

4. See the WHO Statement on the Meeting of the IHR Emergency Committee Meeting concerning the international spread of wild poliovirus, 5 May 2014. Retrieved from the WHO website: www.who.int/mediacentre/news/statements/2014/polio $-20140505 / \mathrm{en} /$

5. See EU Response to Ebola. Retrieved from European Newsroom website: europa. eu/newsroom/highlights/special-coverage/ebola/index_en.htm\#1

6. See "About the International Health Regulations". Retrieved from the wHO website: www.who.int/ihr/about/en/

7. See http://ebola.itg.be/en/faq/ 


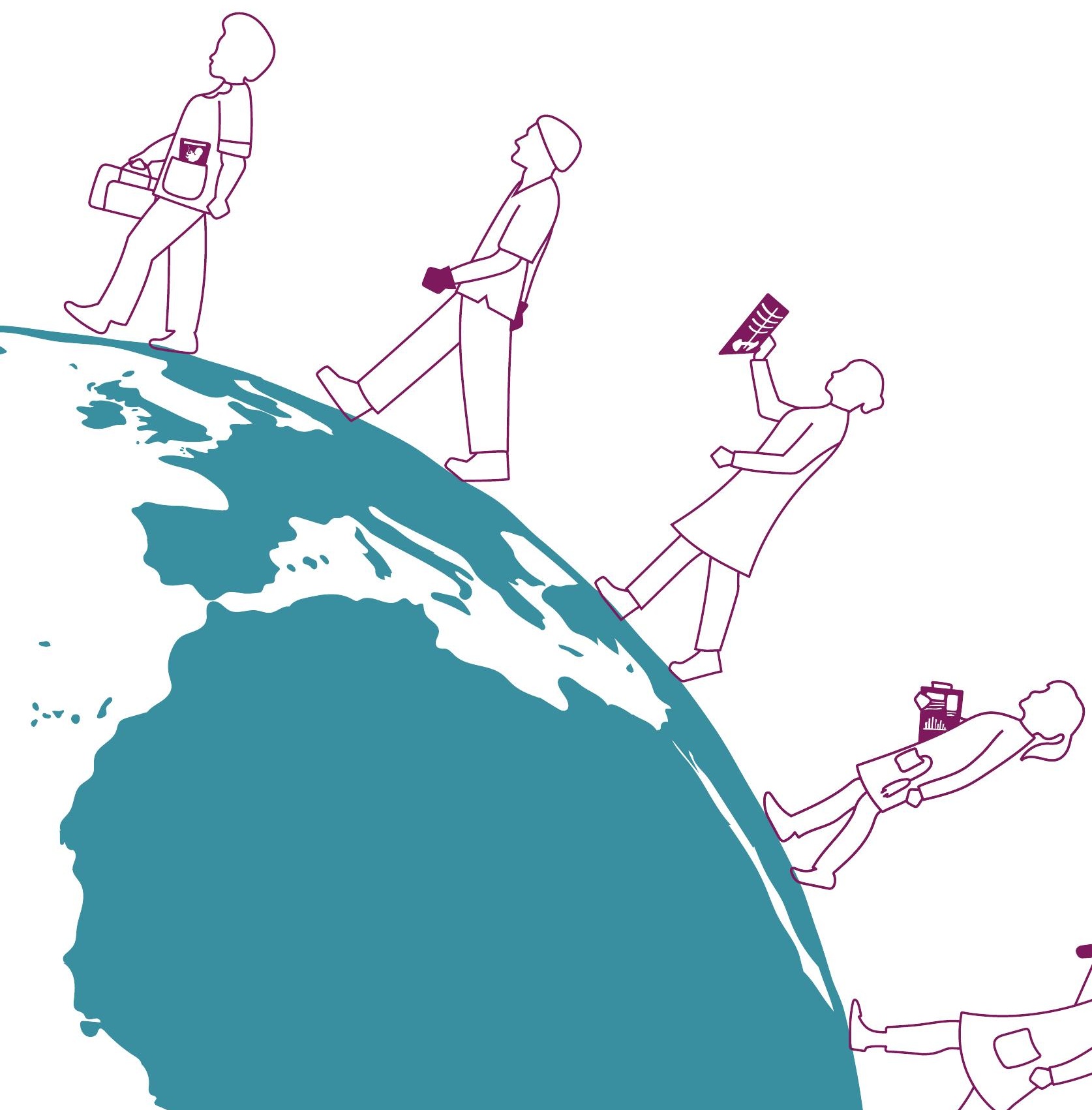




\section{3}

\section{Interrogating resilience in health systems development}

van de Pas, R., Ashour, M., Kapilashrami, A., \& Fustukian, S. (2017).

Interrogating resilience in health systems development.

Health policy and planning, 32(suppl_3), iiis8-iii90

doi: 10.1093/heapol/czx110 


\begin{abstract}
The Fourth Global Symposium on Health Systems Research was themed around 'Resilient and responsive health systems for a changing world.' This commentary is the outcome of a panel discussion at the symposium in which the resilience discourse and its use in health systems development was critically interrogated. The 2014-15 Ebola outbreak in West-Africa added momentum for the wider adoption of resilient health systems as a crucial element to prepare for and effectively respond to crisis. The growing salience of resilience in development and health systems debates can be attributed in part to development actors and philanthropies such as the Rockefeller Foundation. Three concerns regarding the application of resilience to health systems development are discussed: (1) the resilience narrative overrules certain democratic procedures and priority setting in public health agendas by 'claiming' an exceptional policy space; (2) resilience compels accepting and maintaining the status quo and excludes alternative imaginations of just and equitable health systems including the sociopolitical struggles required to attain those; and (3) an empirical case study from Gaza makes the case that resilience and vulnerability are symbiotic with each other rather than providing a solution for developing a strong health system. In conclusion, if the normative aim of health policies is to build sustainable, universally accessible, health systems then resilience is not the answer. The current threats that health systems face demand us to imagine beyond and explore possibilities for global solidarity and justice in health.
\end{abstract}

\title{
Key Messages
}

The resilience narrative overrules certain democratic procedures and priority setting in public health agenda's by 'claiming' an exceptional policy space.

Resilience forces accepting and maintaining of the status quo and excludes alternative imaginations of just and equitable health systems.

Resilience is symbiotic with, and not a solution to, the vulnerability of societies and health systems. 


\section{INTRODUCTION}

The Fourth Global Symposium on Health Systems Research (HSR) (Health Systems Research 2016) was themed around 'Resilient and responsive health systems for a changing world.' As a key reflection, the Vancouver statement recognizes the many meanings of resilience. It argues that 'Systems need to be resilient precisely so that the burden of such resilience does not fall on the most vulnerable in our societies' (HSR 2016). We contest the logic of this approach as this opaque description of resilience provides a smokescreen for the acceptance of a policy discourse that has ideological bearings and political implications. This commentary is the outcome of a panel discussion at HSR (2016) in which the resilience discourse and its use in health systems development was critically interrogated. We argue that resilience is symbiotic with, and not a solution to, the vulnerability of societies and health systems.

\section{DISCUSSION}

The resilience concept has entered the health systems domain only recently. The 2014-15 Ebola outbreak in West-Africa added momentum for the wider adoption of resilient health systems as a crucial element to prepare for and effectively respond to crisis. In this reading, resilience is comprised of a pre-existing strong health system as well as its ability to react in a suitable and timely response to an outbreak. (Marie-Paule Kieny et al. 2014) Surprisingly, there has been little attention to a crucial pre-condition of resilience: that it presumes that crises are permanent or a given in the 'changing world' in the title of HSR (2016). Global health risks, potentials for crisis and related vulnerabilities are not evenly distributed. The Ebola outbreak indicated that these health risks follow structural injustices, neglect and render invisible the people most affected by such global risks. People dealing with acute health challenges form the background, or are peripheral, in the narrative on health systems resilience. (Nunes 2016)

The growing salience of resilience in development and health systems debates can be attributed in part to development actors and philanthropies such as the Rockefeller Foundation. They have been key in framing the discourse as relevant for health systems development. The concept of 'resilience dividend' introduced by the Foundation's president, Rodin (2013), was subsequently argued as a key health systems function in the global debate following the lessons of the Ebola outbreak. In their framework of a resilient health system, Kruk et al. (2015) identify five key characteristics/elements that health systems should espouse: being aware, diverse, self-regulating, integrated, and adaptive. Blanchet et al. (2017) have also put forward 
a conceptual framework to manage resilience of health systems. This framework, firmly grounded in complex systems sciences, identifies four interlinked dimensions to manage resilience: knowledge, uncertainties, interdependency, and legitimacy.

A significant validation of the concept came from the World Health Organization (WHO) as it incorporated resilience as an objective in its health systems strengthening and research portfolio (Kutzin and Sparkes 2016) although it is amiss in the formal resolutions passed by the wHO's governing bodies in 2016. (World Health Organization 2016c) This inconsistency may reflect an unease or lack of consensus among country delegations on what the concept offers in advancing the global public health agenda.

Roemer-Mahler and Elbe (2016) have described the crisis discourse in their study on pharmaceutical developments and the securitization of health in the Ebola response. Such securitization, they argue, has also created an exceptional political space in which pharmaceutical development can be freed from constraints....to break key norms and rules governing the development and approval of drugs and vaccines. (Roemer-Mahler and Elbe 2016, p. 487)

The resilience narrative similarly overrules certain democratic procedures and priority setting in public health agenda's by ‘claiming' an exceptional policy space.

In the opening plenary of HSR (2016), the WHO's Agnes Soucat mentioned that the emergence of resilience after the Ebola outbreak could be somehow regarded as the '9/11 of global health'. Neocleous (2015) argues that the rise of the resilience discourse in the last 15 years is indeed connected to the security problematic of societies. In total 9/11 made it clear that for major state powers security challenges can no longer be externalized (e.g. via armed conflict with an enemy state) but that an internalized narrative is required. Basically, resilience intimately connects the security and emergency concept and solidifies a mode of self-governance that, in the Foucauldian sense of bio-politics and governmentality, is an ideal, modern and appropriate method to manage populations. (Foucault 2009) Resilience has become the leitmotiv for a broad range of policies, which demand populations to anticipate and prepare for future catastrophes and survive. This catastrophe is often defined in relation to an economic crisis, a natural/climatic disaster, a terrorist attack or a health emergency. Regardless of the nature of the catastrophe and its underlying factors, 'vulnerable' individuals and communities are encouraged to withstand, and even bounce-back from these events. (Neocleous 2015)

Indeed it even demands a certain exposure to the threat before its occurrence so that we can be better prepared. Resilience as such appears to be a form of immunization. (Brad Evans and Reid 2015, p. 156) 
We argue that resilience forces an acceptance and maintenance of the status quo and, as Neocleous (2015) asserts, excludes alternative imaginations of just and equitable health systems including the socio-political struggles required to attain those.

In the context of health systems development in times of global instability, resilience can be seen as a political strategy to attain 'good enough governance' for health (Drezner 2013) and not to tackle the structural crisis weakening health systems.

Joseph (2013) argues that resilience is best understood in the context of rolling-out neoliberal governmentality. Post-liberal theories such as those on complexity accept as given the idea that governance has become de-politicized and that terms such as resilience are here to stay, without critical reflection. Chandler (2014) confirms that resilience approaches develop upon and transform neoliberal conceptions of complex systems.

Resilience is a shallow and shifting concept, and has varying meanings depending on which discipline is applying it. It might hence be exchanged easily with another buzzword. The resilience discourse might increasingly pervade international organizations like the wHO but this does not necessarily lead to a meaningful effect or transformational change on the ground. (Joseph 2013)

The empirical case study from Gaza presented in the panel further evidences our critique on resilience maintaining the status-quo of social injustices. (Ashour 2016) Although vulnerability and resilience are often thought of as two sides of a coin, in the Gaza Strip, resilience and vulnerability are actually interrelated. The international community (and humanitarian sector) has intervened routinely since the start of the conflict in Gaza in 1948. Yet, while the plethora of health initiatives has prevented starvation and disease they have not addressed the conflict or the vulnerability resulting from it. These interventions enable people to survive and to live-but live a bare existence. The health system is far from dysfunctional but the suffering of people continues; people queue for hours to access basic healthcarecreating, in a nutshell, 'resilient' systems for vulnerable populations whose dignity is violated with every interaction with the health system. Resilience is, in some ways at least, their vulnerability and disposability. One must refrain from seeing this model of resilience and vulnerability as an example for health systems in other parts of the world. It would be a step backward. (Ashour 2016)

In the discourse of what resilience 'is' and 'enables' for health systems (Kruk et al. 2015), it is equally important to identify what it 'is not'. It has no built-in 'moral compass'; is stripped of social and political analysis; undermines theory of power, agency and structure; and runs the risk of being co-opted to sustain an undesirable 
status quo of legitimizing chronic weak, under-resourced, health systems. (Institute of Development Studies 2013) With the resilience focus on permanency of crisis, we fail to consider alternative political and moral policy pathways rooted in the principles of social justice, investing in global public goods for health (such as universally accessible health systems) while considering also a central role for the preservation of ecology. (Remco van de Pas 2016)

And these are to be seriously considered. Recent thinking on a reflective and responsible modernity paradigm hint towards the inherent transnational, cosmopolitan, shared responsibility approach required by country governments and other actors to govern civilization and health systems in order to correct the unequal distribution of global health risks. (Beck 2006a) Atkinson's book Inequality: What Can Be Done? describes how increasing international cooperation could create conditions for the establishment of welfare policies in all countries of the world by avoiding the 'tax competition' that erodes government revenue and thus the space for social policy by creating minimum taxation and social policy standards. (Atkinson 2015b)

\section{GONGLUSION}

In conclusion, if the normative aim of health policies is to build sustainable, universally accessible, health systems then resilience is not the answer. The current threats that health systems face, demand us to imagine beyond and explore possibilities for global solidarity and justice in health by acknowledging that the future of the world's population is closely connected through our shared risks and capabilities to overcome modern health challenges. 


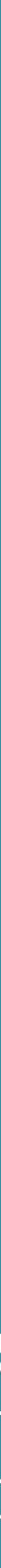




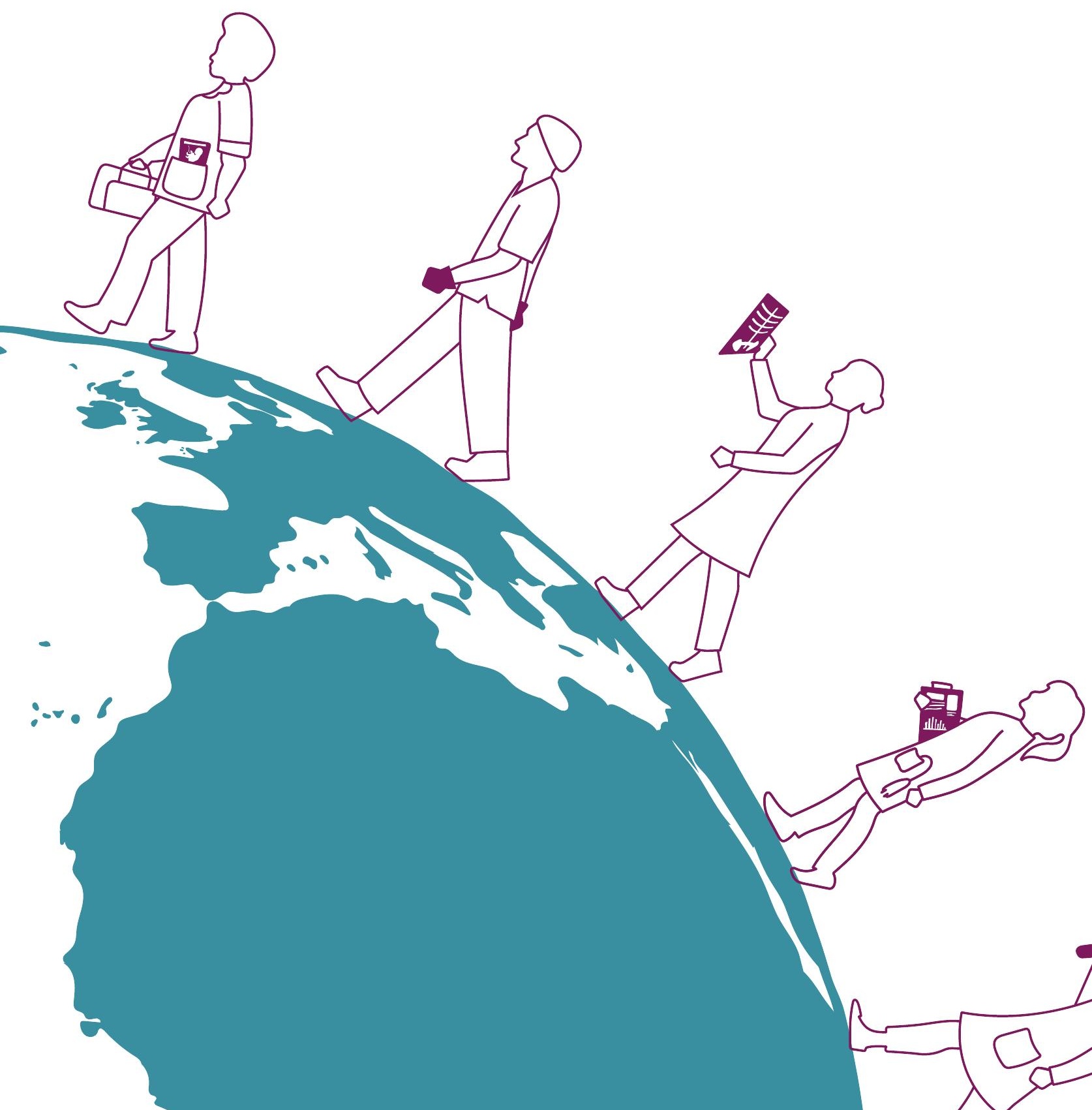




\section{1}

\section{Global health governance in the sustainable development goals: Is it grounded in the right to health?}

van de Pas, R., Hill, P. S., Hammonds, R., Ooms, G., Forman, L., Waris, A., Brola, C. McKee M., Sridhar, D. (2017).

Global health governance in the sustainable development goals:

Is it grounded in the right to health?

Global Challenges, 1(1), 47-60.

doi: 10.1002/gch2.1022 


\begin{abstract}
This paper explores the extent to which global health governance-in the context of the early implementation of the Sustainable Development Goals is grounded in the right to health. The essential components of the right to health in relation to global health are unpacked. Four essential functions of the global health system are assessed from a normative, rights-based, analysis on how each of these governance functions should operate. These essential functions are: the production of global public goods, the management of externalities across countries, the mobilization of global solidarity, and stewardship. The paper maps the current reality of global health governance now that the post-2015 Sustainable Development Goals are beginning to be implemented. In theory, the existing human rights legislation would enable the principles and basis for the global governance of health beyond the premise of the state. In practice, there is a governance gap between the human rights framework and practices in global health and development policies. This gap can be explained by the political determinants of health that shape the governance of these global policies. Current representations of the right to health in the Sustainable Development Goals are insufficient and superficial, because they do not explicitly link commitments or right to health discourse to binding treaty obligations for duty-bearing nation states or entitlements by people. If global health policy is to meaningfully contribute to the realization of the right to health and to rights-based global health governance then future iterations of global health policy must bridge this gap. This includes scholarship and policy debate on the structure, politics, and agency to overcome existing global health injustices.
\end{abstract}




\section{INTRODUCTION}

As governments pursued the Millennium Development Goals (MDGs), the idea of global health featured increasingly in health policy literature. Academic debate has sought to define it, differentiating it from international health (Fried et al. 2010; Koplan et al. 2009), to assert its position within a public health epistemology (Theodore M Brown et al. 2006), to argue for its distinctive complexity (Hill 2011), and to contest its framing as a recent and novel phenomenon. (Fidler 2001) During this time, its use has increased exponentially, being used by public and private stakeholders, in networks and alliances, and diverse relationships, leading Kickbusch and Szabo to characterize it as a "global public health domain" (Kickbusch and Szabo 2014), with key health challenges faced by the international community being recast as issues of governance rather than disease. (Kickbusch 2006)

The management of this rich interdependence of actors, networks, and interfaces demands fresh imagining of governance. Fidler's inclusive definition of global health governance as "the use of formal and informal institutions, rules, and processes by states, intergovernmental organizations, and non-state actors to deal with challenges to health that require cross-border collective action to address effectively" (Fidler 2010), has been parsed further by Kickbusch and Szabo. (Kickbusch and Szabo 2014) They distinguish three global health governance concepts:

- global health governance, focusing on institutions and processes of global governance with an explicit health mandate such as the World Health Organization (WHO) or the Global Fund to fight AIDS, Tuberculosis, and Malaria (Global Fund);

- global governance for health, that embraces institutions and processes with direct or indirect impact, including the United Nations (UN), and the World Trade Organization (WTO); and

- governance for global health, referring to the mechanisms and institutions created at national and regional levels to support global health governance. (Frenk and Moon 2013)

But, as Frenk and Moon point out: "Global governance is distinct from national governance in one critical respect: there is no government at the global level." (Frenk and Moon 2013) There is a largely unchallenged acceptance of the Westphalian arrangement of populations into nation states, but as of yet no equivalent consensus around a "hierarchical political authority, or world government" with authority over them. (Fidler 2010) 
If there were such a government, Owen Barder would characterize it as a failed state - as he did in a recent presentation to the London School of Economics' Diplomacy Commission:

no rule of law with no institutions to set or enforce rules, and no way to agree and enforce contracts... no mechanism to raise money for, or to deliver effectively, public goods such as clean air, law and order, financial stability, public infrastructure, research, and development or disease surveillance... a winner-takes-all economy... with no collective insurance for its citizens against natural disasters, and in which inequality is allowed to grow to the extent where the rich have to wall themselves off from the poor. (Barder 2014)

The critique is not without substance. Yet, however imperfect, the nation state remains the primary locus of political legitimacy and the pursuit of justice. Indeed, as the recent referendum on the United Kingdom's European Union (EU) membership, as well as the growth of anti-European parties in France and other parts of Europe shows, there is evidence of a retreat from supranational structures.

Thomas Nagel has argued that the path from global anarchy-the absence of global authority-to global justice will not always be equitable. (Nagel 2005) It is through the expansion of complex multilateral networks and supranational arrangements between those states, initially pursuing common interests rather than altruistic sacrifice, that global governance arrangements will become institutionalized. To apply this to global health: it is likely that the global institutions that emerge may lack legitimacy, and by prioritizing the interests of its major funders (both states and non-states) may distort distributive justice-a key critique of global health philanthropists or global public-private initiatives for health. (Stuckler et al. 2011) The moral and public imperative will be to democratize and hold accountable such institutions in order to enhance their legitimacy. (Stuckler et al. 2011)

It is in this context that Frenk and Moon identified four essential functions of the global health system, that we will argue, parallel several key functions of the state: the production of global public goods, the management of externalities across countries, the mobilization of global solidarity, and stewardship. (Fidler 2010) With the recent UN acceptance of the Sustainable Development Goals (SDGs), ${ }^{1}$ governance of that global health system is increasingly important. The sDGs are universal in nature, integrating economic and social development, and environmental change, with broad implications for global health. Sustainable Development Goal 3 (SDG 3) "Ensure healthy lives and promote well-being for all at all ages" extends its claim

1 United Nations Sustainable Development Goals. UN: New York 2015. https://sustainabledevelopment.un.org/topics. 
from the unfinished agenda of the MDGs to include additional communicable disease targets, but also to address non-communicable disease, mental health and well-being, motor-vehicle trauma, and the health consequences of environmental pollution. In fact, SDG 3 arguably embraces all the dominant contributors to the global burden of disease. (Global Burden of Disease Study 2013 Collaborators 2015) This near comprehensive scope, with its increasing engagement with sustainable change in other sectors, the demands SDG 3 sets for all states, and the demand for solidarity between them-makes the achievement of a system of global governance for health an imperative.

In our research, examining the positioning of health within the emerging post-2015 SDGs, the Go4Health (Goals and Governance for Health in the Post-2015 Agenda) research team has already argued that the global goal for health must be grounded in the right to health. (Ooms et al. 2013) However, while the right to health may be implicit in the aspirations of Universal Health Coverage (UHC) (Ooms et al. 2014), the global health governance that will respond to the complex demands of the SDGs is yet to emerge.

In this paper, we will explore the extent to which global health governance-in the context of the early implementation of the SDGs-is grounded in the right to health. First, we will unpack the essential components of the right to health in relation to global health. We will then outline Frenk and Moon's four functions - reordered for the purposes of this analysis of global health governance, and considered in the light of the right to health - and conduct a normative analysis of how each of these governance functions should operate. We will then map the current reality of global health governance now that the post-2015 SDGs are beginning to be implemented -a picture that may share elements of Barder's caricature - pointing to the incremental but achievable steps that are needed as we launch onto "[t]he road to dignity by 2030: ending poverty, transforming lives and protecting the planet". ${ }^{2}$

\section{Defining the Right to Health in the Context of the SDGs}

By the right to health, we are referring to the entitlement of all humans to organized efforts by society that promote and improve health and the corresponding obligations born by governments and the international community, as enshrined in international human rights law. (Ooms et al., 2014) While several treaties have addressed the right to health, our primary reference is the International Covenant on Economic, Social and Cultural Rights (ICESCR) (United Nations General Assembly: International

2 United Nations Secretary-General. The road to dignity by 2030: ending poverty, transforming lives and protecting the planet. Synthesis Report of the Secretary-General on the post-2015 sustainable development agenda. New York, United Nations, (2014). http://www.un.org/ga/search/view_doc.asp? symbol=A/69/700\&Lang=E 
Covenant on Economic, Social and Cultural Rights, 1966). This is because of its broad endorsement by states and wider scope than treaties that focus on the right to health for specific groups - like the Convention on the Rights of the Child or the Convention on the Elimination of All Forms of Discrimination against Women. The ICESCR affirms "the right of everyone to the enjoyment of the highest attainable standard of physical and mental health" (Article 12(1)) and the responsibility of every state "to take steps, individually and through international assistance and co-operation, especially economic and technical, to the maximum of its available resources, with a view to achieving progressively the full realization of" the right to health (Article 2(1)) (United Nations Committee on Economic, Social and Cultural Rights, 2003). These articles have been interpreted to codify rights to both adequate health care and the underlying determinants of health, and to place corresponding obligations on governments to act on health at home and where able, abroad (United Nations Committee on Economic, Social and Cultural Rights, 2000).

A right to health framed global governance for health would need to ensure at least "minimum essential levels of each of the rights enunciated in the Covenant, including essential primary health care"- rights expressed in the proposed SDGs as the achievement of UHC "including financial risk protection, access to quality essential health care services, and access to safe, effective, quality and affordable essential medicines, and vaccines for all". ${ }^{23}$ However, the right to health is not the only economic, social, or cultural right impacting on the SDGs' implementation, which also require realization of rights to water and sanitation, food, housing, education, and collectively, to development. This broader scope is exemplified by the content of the inter-related goals that are necessary for the implementation of the health goals: for instance, SDG 2 (End hunger, achieve food security, and improved nutrition and promote sustainable agriculture); SDG 4 (Ensure inclusive and equitable quality education and promote lifelong learning opportunities for all); SDG 6 (Ensure available and sustainable management of water and sanitation for all); SDG 11 (Make cities and human settlements inclusive, safe, resilient and sustainable); SDG 16 (Promote peaceful and inclusive societies for sustainable development, provide access to justice for all and build effective, accountable and inclusive institutions at all level); and SDG 17 (Strengthen the means of implementation and revitalize the global partnership for sustainable development).

\section{Four Functions for Global Health Governance in the Context of the SDGs:}

\section{A Normative Right to Health Analysis}

With their claim to universality, the SDGs do provide a framework within which global health is redefined in terms of the health of the global population, understood in terms of global interdependence. The absence of a world government does not 
obviate the need for global governance, although the form of that governance will clearly be different from the governance of nation states. To facilitate our analysis, we have re-ordered and adapted Frenk and Moon's four functions of the global health system (Frenk and Moon 2013), building on the current functions of nation states that would allow the right to health to be achieved, and extrapolating them to global governance for health:

1. Stewardship provides "overall strategic direction to the global health system" (Frenk and Moon 2013), and embodies in many ways the functions of the executive branch of the state: the establishment of norms, values, and rules that guide the development of policy and setting of priorities, the advocacy for global health across sectors and the convening of partnerships at global and regional level that might enable its achievement.

2. The production of global public goods is instrumental in progressively ensuring "the highest attainable standard of physical and mental health" (Frenk and Moon 2013), and embodies and operationalizes the policy concepts elucidated in the stewardship function. Arguably this parallels the functions of the legislative branch of the state, implementing policy with the resources mobilized domestically and through global solidarity. Frenk and Moon draw particular attention to knowledge-related public goods, research and development, standards and guidelines, comparative evidence, and analyses. We define global public goods more broadly, echoing Kickbusch's call for an expansive concept of global public goods for health (GPGH) which highlights health's "deep relation to human rights, equity and governance" noting they "all relate to the provision of GPGH”. (Kickbusch 2013a)

3. The mobilization of global solidarity combines four major sub-functions: the shared financing of global health; capacity building and technical assistance; humanitarian interventions in crisis; and agency for the marginalized and dispossessed. This function parallels the role of the state in revenue-raising through taxation and other means, coupled with resources provided by global partners, and its disbursement in the implementation of redistributive policies determined through its stewardship functions.

4. The management of externalities embraces those functions that contain the negative impact of decisions made by one state - or transnational body-on others. Frenk and Moon list the deployment of instruments such as surveillance systems, coordination mechanisms, and information channels essential for controlling international risk, but the exercise of sanctions - analogous to the judicial branch of the state, would need to find equivalence at the global level. 
In terms of stewardship, a Right to Health driven Global Health Governance would align its goal with Article 12(1) ICESCR which recognizes "the right of everyone to the enjoyment of the highest attainable standard of physical and mental health" (United Nations General Assembly: International Covenant on Economic, Social and Cultural Rights, 1966). It would achieve this by taking incremental steps "to the maximum of its available resources, with a view to achieving progressively the full realization of the rights recognized in the present Covenant..." (United Nations General Assembly: International Covenant on Economic, Social and Cultural Rights, 1966). But it would define "minimum core obligations" from which this progressive realization would proceed, ensuring "the right of access to health facilities, goods, and services on a non-discriminatory basis, especially for vulnerable or marginalized groups... access to the minimum essential food which is nutritionally adequate and safe, to ensure freedom from hunger to everyone... access to basic shelter, housing and sanitation, and an adequate supply of safe and potable water... [t]o provide essential drugs... [t]o ensure equitable distribution of all health facilities, goods and services... [t]o adopt and implement a national public health strategy and plan of action, on the basis of epidemiological evidence, addressing the health concerns of the whole population". ${ }^{3}$ This non-discriminatory health strategy - under global governance would be "devised, and periodically reviewed, on the basis of a participatory and transparent process". ${ }^{25}$

Under a Right to Health based system of global health governance, the production of global public goods would necessarily prioritize meeting those "minimum core obligations", delivering the knowledge-related public goods that ensure universal access to effective curative and preventive health services and the essential public health provisions anticipated in the ICESCR. ${ }^{25}$

The mobilization of global solidarity would be integral to the achievement of this. Under the Right to Health, every state is responsible for ensuring these minimum core obligations "to the maximum of its available resources". ${ }^{4}$ It assumes shared financing of global health as states "take steps, individually and through international assistance and co-operation, especially economic and technical, to the maximum of its available resources", ${ }^{26}$ to achieve progressively the realization of the Right to Health. Shared responsibility to realize the right to health is emphasized in General Comment 14 which suggests that "it is particularly incumbent on States

3 United Nations Committee on Economic, Social and Cultural Rights. General Comment No. 14: The Right to the Highest Attainable Standard of Health, E/C.12/2000/4. Geneva: United Nation. (2000). http://www.refworld.org/ docid/4538838d0.html.

4 United Nations General Assembly: International Covenant on Economic, Social and Cultural Rights, G.A. Res. 2200 A (XXI), 21 U.N. GAOR Supp. (No. 16) at 49, U.N. Doc.A/6316 (1966), 993 U.N.T.S. 3, entered into force. 1976. http://wwwl.umn.edu/humanrts/instree/b2esc.htm. 
parties and other actors in a position to assist, to provide 'international assistance and cooperation, especially economic and technical' which enable developing countries to fulfill their core and [comparable priority obligations]". ${ }^{5}$ Subsequent expert interpretations have emphasized that states hold extraterritorial obligations to enable the realization of "core obligations to realize minimum essential levels of economic, social and cultural rights". (Hammonds et al. 2012; International Commission of Jurists 2012) In capacity building and technical assistance, and in particular in humanitarian interventions in crisis, this shared responsibility is assumed. The "agency for the marginalized and dispossessed", to which Frenk and Moon point is subsumed in the right to health principle of non-discrimination, and in its prioritization of vulnerable and marginalized groups. Here the bar is raised - if a health issue disproportionately affects the marginalized, protection of their interests necessitates a policy response, even if, at a population level, it is not costeffective (or politically palatable at the domestic level). (Ooms et al. 2014)

The management of externalities under a right to health-based global health governance would be implied in the principle of shared responsibility, and interface with the recognition of other cognate rights articulated in the Universal Declaration of Human Rights, ${ }^{6}$ and in the ICESCR (United Nations General Assembly: International Covenant on Economic, Social and Cultural Rights, 1966). This could imply that the legal functions of the wHO, specifically the legislative and executive authority, in addressing global health threats should be strengthened. One can think here of deepening the International Health Regulations (IHR) (Lawrence O Gostin and Sridhar 2014), or even a much further-reaching Framework Convention on Global health, that would serve as a legal umbrella for the further management responsibilities of states to address global "bads" and strengthening GPGH. (Lawrence O Gostin et al. 2013)

\section{Global Health Governance in the Context of the SDGs and the Right to Health}

\section{Stewardship}

In terms of stewardship for global health within the context of the SDGs - setting the global health agenda, establishing norms and guidelines, engaging partners for international policy development and implementation - the wHO is unique

5 United Nations Committee on Economic, Social and Cultural Rights. General Comment 14: The right to the highest attainable standard of health. (Twentysecond session, 2000), U.N. Doc. E/C.12/2000/4 (2000), reprinted in Compilation of General Comments and General Recommendations Adopted by Human Rights Treaty Bodies, U.N. Doc. HRI/GEN/1/Rev.6 at 85 (2003).

http://wwwl.umn.edu/humanrts/gencomm/escgencoml4.htm.

6 United Nations General Assembly: Universal Declaration of Human Rights, G.A. res. 217A (III), U.N. Doc A/810 at 71. 1948. Available from: http://wwwl. umn.edu/humanrts/instree/bludhr.htm. 
in terms of its legitimacy as the only global health institution with a mandate to promulgate international law. (Moon et al. 2010) Health goal SDG 3 "Ensure healthy lives and promote well-being for all at all ages" echoes both the right of everyone to "highest attainable standard of physical and mental health", ${ }^{26}$ and the wHO's definition of health as a "state of complete physical, mental and social well-being and not merely the absence of disease or infirmity". There is clear synchrony in aspiration. But despite the representation of nation states through their ministers of health in the World Health Assembly (WHA), and respect for its norm-setting functions, the capacity of the WHO to embody the stewardship function of global governance for health is repeatedly questioned. (Hoffman and Røttingen 2014; Ruger and Yach 2009) Substantially underresourced, and operationally hamstrung, the WHO faces a situation where the bulk of its budget is earmarked by powerful "donor" states. Sridhar and Woods have phrased this institutional gridlock as "trojan multilateralism", defined as "increased funding to multilateral institutions that is creating the illusion of multilateral intent, whereas it is covertly introducing bilateral goals and interests into multilateral institutions". (Sridhar and Woods 2013)

As a consequence, the wHO is constrained in terms of policy and direction, and there are equivocal perceptions of its capacity to drive the global health agenda. This was most recently evident in the critiques of its executive role in and leadership response to the Ebola outbreak (Lawrence O Gostin et al. 2014), and again in its failure to secure UHC as the overall SDG health goal. (Brolan and Hill 2016) At the same time, recognition of the centrality of the wHO to global health governance is evident in proposals for a Committee $\mathrm{C}$ which would allow the wHO to more effectively engage civil society, formalizing civil society's current significant contribution to global health governance. (Kickbusch et al. 2010) Yet recent proposals for a new UN agency to address global health (Dybul et al. 2012), revisit earlier proposals to extend the Global Fund from its targeted communicable disease mandate to become a Global Fund for Health (Cometto et al. 2009), and an earlier UN decision that relocated management of the HIV epidemic from the wHO into the Joint United Nations Programme on HIV/AIDS (UNAIDS). ${ }^{8}$

But the wHO has not held a monopoly on the stewardship for global health for some time: the UN agencies UN Children's Fund (UNICEF), UN Population Fund (UNFPA), and UNAIDS have specific global health mandates that interface with the wHO.

7 Preamble to the Constitution of the World Health Organization as adopted by the International Health Conference, New York, 19-22 June, 1946; signed on 22 July 1946 by the representatives of 61 States (Official Records of the World Health Organization, no. 2, p. 100) and entered into force on 7 April 1948. http://www.who.int/governance/eb/who_constitution_en.pdf.

8 UNAIDS. (2016) http://www.unaids.org/. 
Since the 1990s, the World Bank has also made the claim for investing in health. ${ }^{9}$ The WTO exercises a governance role for medicines through the Agreement on Trade-Related Aspects of Intellectual Property Rights (TRIPS). ${ }^{10}$ The Organization for Economic Cooperation and Development (OECD)-initially the provenance of high-income economies-has now redefined aid effectiveness (including for health) into development effectiveness, reaching beyond its immediate membership and embracing the multiple, complex contributors to global health and development. (Busan Partnership Agreement 2011)

What is increasingly clear is that there will be no return to an imagining of a global governance hierarchy and that the concrete, architectural metaphors of the past no longer suffice. (Fidler 2009) Global health governance will continue to be networked, with largely voluntary partnerships and alliances addressing key issues as they have in the Gavi Alliance, Global Fund, Roll Back Malaria, The Partnership for Maternal, Newborn \& Child Health, and the NCD-Alliance. Under the right to health, coordination of the networks would itself be a necessary function, the global policies regularly and transparently reevaluated (United Nations Committee on Economic, Social and Cultural Rights, 2003). This option has been canvassed in the form of a Global Health Forum, offering voice to multiple stakeholders, beyond the state representation of the UN system. ${ }^{11}$ The conspicuous consultation of the "World We Want" campaign ${ }^{12}$ was a direct response to civil society's absence from the formulation of the MDGs.

The experience of the MDGs is that, once accepted, the goals and their targets are relatively fixed. Despite their significant contribution to the Global Burden of Disease (Murray et al. 2012), the non-communicable diseases (NCDs) were marginalized for the 15 years of the MDGs. Although NaDs are now included in the SDGs, it is to be seen whether they will receive the prominence and attention deserved. The NCD challenge does not only require funding or new financing mechanism, but also global regulation to address the key vectors of the epidemic, such as the overconsumption of sugars, tobacco, and alcohol. The global governance structures as part of the sustainable development agenda are poorly suited to deal with this multisectoral issue. (Sridhar et al. 2013)

\footnotetext{
9 World Bank. Pandemic Emergency Financing Facility: Frequently Asked Questions (2016) http:// www.worldbank.org/en/topic/pandemics/brief/pandemicemergency-facility-frequently-askedquestions.

10 World Trade Organization (WTO) -Agreement on Trade-Related Aspects of Intellectual Property Rights (TRIPS Agreement). (1994) http://www.wipo.int/wipolex/en/other_treaties/details.jsp?treaty_ id $=231$.

11 WHO World Health Forum. Concept paper. (2011) http://www.who.int/dg/reform/en_who_reform_world_health_forum.pdf. 12 United Nations. World We Want. (2016). https://www.worldwewant2030.org/.
} 
The MDG 5b "Achieve, by 2015, universal access to reproductive health" was only added in 2007 following persistent community protest. The SDGs may provide for the progressive realization envisaged in the right to health, but experience from the MDGS suggests the SDG indicators currently under development will determine the priorities for implementation, and as with the MDGs, will form the hubs around which governance structures will coalesce. In terms of ensuring support for the minimum core obligations and prioritizing the marginalized and vulnerable, this comes with some risks.

The recent Ebola crisis provides some insight into potential processes: in its aftermath, the WHO's Report of the Interim Ebola Assessment Panel recommended support for national and international capacity to implement its IHR-but recognizing the reluctance of Member States to raise their contributions, ${ }^{13}$ recommended a modest the wHO Emergency Contingency Fund and a process of internal reform. ${ }^{14}$ Concurrently, the World Bank Pandemic Emergency Financing Facility has been proposed (World Bank, 2016). The facility will provide financial resources to deploy trained health workers, equipment, medicines, and whatever else is required quickly when a pandemic hits. Simultaneously, the Global Health Security Agenda, driven by the Centers for Communicable Disease Control and Prevention and related United States' agencies, has been created in partnership with other nations, international organizations and public and private stakeholders, to "seek to accelerate progress toward a world safe and secure from infectious disease threats and to promote global health security as an international security priority." ${ }^{15}$ Each of these initiatives is a rational response to a significant issue for global health security. Each would have to recognize the legitimacy of other contributions. Yet the lack of a single locus for governance responding to this threat is of concern, and risks the duplication of effort and the disruptive lack of coordination that has characterized other acute crises. And while Ebola is a tragic threat for Sub-Saharan Africa, the significant investment for its control from key development donors cannot be considered proportional compared with other global health burdens. But even within the response to Ebola, while accelerated investment in vaccine development has been highlighted, the health systems deficits identified as underlying the outbreak may not obtain the urgent financial and technical attention required. A short-term focus on a vaccine may deflect commitment from the long term support necessary to address the lack of development and coherence between elements of the systems

13 TWN Info Service on Health Issues. Health: WHO D-G warns of serious funding shortfalls in 2016-17 budget. Published in SUNS \#8346 dated 2 November (2016) Third World Network. http://www.twn.my/title2/health.info/ 2016/hil61102.htm.

14 WHO. Report of the Interim Ebola Assessment Panel. (2015) WHO: Geneva http://www.who.int/csr/resources/publications/ebola/report-by-panel.pdf.

15 US Government .The Global Health Security Agenda. (2015)

http://www. globalhealth.gov/global-health-topics/global-health-security/ghsagenda.html. 
building blocks-the health workforce (HWF), health financing mechanisms, governance and stewardship, and health information systems. (Lawrence O Gostin and Friedman 2015) The policy and governance responses addressing global health security will be amongst many arenas where the competing interests in networked governance may challenge that essential stewardship function that would protect the right to health values.

The right to health concept of non-discrimination also appears to differ from the commitment to address inequality foreshadowed by the sDGs. From the report of the High Level Panel, ${ }^{16}$ the dictum "leave no one behind" has been one of the "transformative elements" of the SDGs, articulated in SDG 10 "Reduce inequality within and among countries". ${ }^{23}$ It is also included in other goals such as SDG 5 "Achieve gender equality and empower all women and girls". ${ }^{23}$ But marginalized and vulnerable populations have not been explicitly identified within the SDGs and Vandermoortele points to the consequences of the SDGS' primary focus on poverty as the underlying concept of global equity. (Vandemoortele 2015) It is in this concept of equity, however, that the right to health, because of its state-centric orientation, produces unexpected outcomes when applied to global governance. As the analysis of UHC and the Right to Health pointed out (Ooms et al. 2014), while the Right to Health expects rectification of inequalities within states, and the privileging of marginalized groups-arguably including refugees and asylum seekers (Brolan et al. 2015)-it does not apply that expectation between states. The principle of shared responsibility in the Right to Health requires the international solidarity that would ensure a low-income country meets the minimum standard for provision of health services, but at a global level, it does not compellingly articulate expectations of equity beyond that.

\section{The production of global public goods}

The production of global public goods, as we define it in a broader sense, operationalizes the abovementioned stewardship functions, and is instrumental in progressively realizing the Right to Health's "highest attainable standard of physical and mental health"-SDG 3's healthy lives and well-being for all. The norms and guidelines for global health are detailed in SDG 3 targets: achieving UHC, through health systems that are adequately resourced and staffed, and guarantees protection against financial risk, access to quality essential health care services, sexual and reproductive health care, and essential medicines for all. ${ }^{23}$ But what the sDGs and their targets do not do is to articulate a clear set of minimum core obligations. For some targets, absolute levels are asked of each state: in SDG 3.2 all countries are to aim

16 Report of the UN Secretary-General's High-level Panel of Eminent Persons on the Post-2015 Development Agenda. (2013) http://www.un.org/sg/management/pdf/HLP_P2015_Report.pdf. 
"to reduce neonatal mortality to at least as low as 12 per 1,000 live births and under-5 mortality to at least as low as 25 per 1,000 live births"; SDG 3.7 seeks "universal access to sexual and reproductive health-care services, including for family planning, information and education". Other targets are expressed at the global level: SDG 3.1 is specific in aiming for "a global maternal mortality ratio to less than 70 per 100,000 live births", with national targets yet to be established; SDG 3.6 seeks to halve global road traffic deaths within 5 years. But other goals lack sufficient operational definition. ${ }^{23}$ SDG 3.3 unrealistically proposes ending epidemics of communicable diseases; SDG 3.4 the promotion mental health and well-being, but without specifying a level of achievement; SDG 3.5 broadly advocates strengthening the prevention and treatment of substance and alcohol abuse; SDG 3.9 seeks to substantially reduce morbidity and mortality from pollution - again without quantification. And while the targets have expanded on the narrower MDG focus, they are not comprehensive. For example, it has been argued that the use of "premature" mortality diminishes the attention given to older people. (Lloyd-Sherlock et al. 2016) A defined set of minimum core obligations, required to satisfy the Right to Health, is not set by the SDGs, although it may be implied in SDG 3.8 "Achieve UHC". By extension, the tracking of progressive realization of those elements that are ultimately operationalized in the SDGs will be limited to those that benefited from indicators agreed by the Inter-agency and Expert Group on Sustainable Development Goal Indicators (IAEG-SDGs).

Sridhar and colleagues have elaborated on the indicators required for UHC monitoring in the SDGs that would cover the six legal principles of the right to health. (Sridhar et al. 2015) These six principles include the progressive realization of the Right to Health and fulfillment of the minimum core obligations, cost-effectiveness, non-discrimination, shared responsibility, participatory decision making and attention to vulnerable, and marginalized groups. Ooms and colleagues have assessed the UHC framework as being in line with the legal principles of progressive realization. The non-discrimination principle is addressed via the development of a health system that is accessible to all, including financially accessible at the point of service. The cost-effectiveness principle might be addressed if UHC follows national determined sets of health services. However, participatory decision making and prioritizing marginalized and vulnerable groups are only included to a limited extent in the UHC framework and its indicators. The biggest difference is that the Right to Health principles of minimum core obligations and shared responsibility, in the form of international financial assistance, receive no attention in UHC policies. (Ooms et al. 2014)

The IAEG-SDGs has developed an indicator framework for the monitoring of the goals and targets of the post-2015 development agenda at the global level, and to support 
its implementation. ${ }^{17}$ The World Bank and the wHO consider it critical to have two indicators on the UHC target 3.8; one of the coverage of interventions, and one on financial protection, both with an explicit equity dimension. ${ }^{18}$ The World Bank and the WHO have released a first global UHC monitoring report in 2015 that is built around these two main indicators. The coverage indicator looks both to prevention services and treatment while their proposed financial protection indicator was built around two sub-indicators: the incidence of impoverishment resulting from out-of-pocket (OOP) health payments, and the incidence of financial catastrophe from the same cause..$^{19}$ But to the dismay of civil society organizations, and also the WHO and the World Bank, the IAEG-SDGS has suggested changing the SDG 3.8 financial protection indicator in "number of people covered by health insurance or public health system per 1,000 population", an indicator that is not a valid measure of financial risk protection and could hide existing health inequalities in countries. (Ravelo 2016)

The research and development of vaccines and medicines, an essential global public good for securing essential medicines for all, is the explicit focus of SDG $3 \mathrm{~b}$, and recurs in SDG 3.8 "Achieve UHC". The explicit inclusion of the TRIPS agreements and the flexibilities to protect Low- and Middle-Income Countries, speaks to an increasingly contested arena for pharmaceutical research, production, and access, in debates on trade partnerships such as the Trans-Pacific Partnership (TPP), ${ }^{20}$ and renewed calls for tiered pricing of drugs. (Owain D Williams et al. 2015) Public health scholars have argued for a Global Biomedical Research \& Development (R\&D) Fund that would address Anti-microbial Resistance, emerging infectious diseases, and neglected diseases, incorporating financing and coordination mechanisms $\mathrm{D}$ that deliver both innovation and access to medicines and technology by the poor. (Balasegaram et al. 2015)

Implicit in these governance and policy proposals are issues of cost and costeffectiveness, and implications for availability, accessibility, acceptability, and quality. While the preamble to the SDGs envisages "a world of universal respect for human

17 UN DESA's Statistics division. Second meeting of the Inter-agency and Expert Group on Sustainable Development Goal Indicators. (2016) http:// unstats.un.org/sdgs/meetings/iaeg-sdgsmeeting-02.

18 UN DESA's Statistics division. Second meeting of the Inter-agency and Expert Group on Sustainable Development Goal Indicators. Statement on SDG 3 Joint Statement by WHO and UNICEF on behalf of health agencies. (2016)

http://unstats.un.org/sdgs/files/meetings/iaeg-sdgs-meeting-02/Statements/UNSSO\%20statement. Goal\%203\%20-\%200ct\%202015.pdf

19 World Health Organization \& World Bank. Tracking universal health coverage: first global monitoring report. (2016) http://apps.who.int/iris/bitstream/10665/174536/1/9789241564977_eng. pdf?ua=1

20 Trans-Pacific Partnership. International trade association. (2016) http:// www.trade.gov/fta/tpp/. 
rights and human dignity, the rule of law, justice, equality, and non-discrimination”. ${ }^{24}$ The Right to Health principle of non-discrimination, while applying within state jurisdictions, has not been extrapolated to apply between them.

Another public good required on the path to UHC is the HWF: SDG $3 \mathrm{C}$ mentions to substantially increase health financing and the recruitment, development, training, and retention of the HWF in developing countries, especially in least-developed countries and small island developing states. Despite the recognition since the early 2000s that the HWF is a crucial bottleneck in attaining the health-related MDGs - and the concerted commitment to building that workforce - the global HWF gap has grown, with a current estimated global deficit of 7.2 million health workers. Because of demographic and epidemiological changes, this deficit is expected to grow to 12.9 million health workers by 2035 (Sidibé and Campbell 2015), further accentuated by maldistribution and urban bias. The same governance debate developed in relation to access to essential medicines ${ }^{21}$ needs to happen for equitable distribution and just policies for HWF development.

Global public goods in terms of knowledge generation have been acknowledged in the call for a data revolution to underpin the monitoring and reporting functions for the SDG indicators. ${ }^{22}$ SDG 16, promoting peaceful and inclusive societies for sustainable development, includes the provision of legal identity for all, dependent on comprehensive vital registration systems. The Global Burden of Disease report has been useful in quantifying health priorities and will continue to play a role in monitoring global change. (Rudan and Chan 2015) The IN-DEPTH network will provide an evolving platform for monitoring SDG indicators. (INDEPTH Network 2016) The systematized evaluation of other health systems evidence through metaanalyses such as the Cochrane collaboration and the Health Observatories' Health in Transition reports are a necessary complement for understanding change. (European Observatory on Health Systems and Policies 2016)

\section{The mobilization of global solidarity}

The mobilization of global solidarity combines four major sub-functions: development financing; technical cooperation and capacity building; humanitarian interventions in crisis; and advocacy - and agency - for the marginalized and dispossessed. While the other three sub-functions are likewise important, a major focus for activities seeking to mobilize global solidarity should be the creation of a just form of financial redistribution between richer and poorer societies.

21 The United Nations Secretary-General's High-Level Panel on Access to Medicines (2016).

Available at: $\underline{h t t p}: / / w w w . u n s g a c c e s s m e d s . o r g /$ \#homepage-l.

22 UN Data revolution group (2016) Available at http://www.undatarevolution.org/. 
In the context of the SDGs, the recent Third International Conference on Financing for Development (FfD3) offers some insight into proposed financing of the SDGs as a whole ${ }^{23}$ although the estimated SDG envelope is well beyond current projections. (Bustreo 2015) The dominant focus is on increasing domestic resourcing, "through modernized progressive tax systems, improved tax policy, and more efficient tax collection". (Global Policy Watch 2015) Illicit financial flows and corruption are targeted; international tax cooperation to be "scaled up" with emphasis being placed on public-private partnerships but stopping short of a global institution to govern international tax issues and their fair share across the globe. The roles of the private sector - effectively directed towards an alignment with sustainable development and the contribution of migration and empowerment of women are noted. In its state-centric orientation, it is taking the first baby step towards being consistent with the right to health's obligation for the state to "take steps, individually and through international assistance and co-operation, especially economic and technical, to the maximum of its available resources" (United Nations General Assembly: International Covenant on Economic, Social and Cultural Rights, 1966).

But is the shared responsibility sufficiently addressed, or are we witnessing in this expanded development agenda a reluctance to sustain-let alone extend-current development assistance? The FfD3 report reiterates the need for providers of Official Development Assistance (ODA) to re-commit to their target of $0.7 \%$ of Gross National Income-more honored in the breach than the observance - and welcomes the additional resources offered by South-South cooperation, and philanthropy. With regards to global health, the contribution of multi-stakeholder partnerships such as Gavi, Global Fund and the Global Financing Facility in support of Every Woman, Every Child are specifically mentioned, together with the wHO's role in directing and coordinating, and its contribution to health systems strengthening and the Framework Convention on Tobacco Control.

But consistent with the analysis of the fourth Organization for Economic Cooperation and Development High Level Forum on Aid Effectiveness in Busan (Fidler 2009), aid is seen as only one contributor to development, with trade and the engagement of the private sector an increasingly dominant counterpart. The lengthy treatment of the WTO in the FfD3-and for health, the reaffirmation of the right to TRIPS flexibilities for low-income countries-suggests some anxiety around the complexity of "global solidarity" that uncritically embraces the private sector.

23 UN 69/313. Addis Ababa Action Agenda of the Third International Conference on Financing for Development (Addis Ababa Action Agenda). (2015) http://www.un.org/ga/search/view_doc. asp?symbol=A/RES/69/313. Flavia Bustreo. Financing the health Sustainable Development Goal. (2015):

http:// www.who.int/life-course/news/commentaries/financing-health-sustainablegoal/en/. 
The global partnership and solidarity of the FfD3 does not live up to the common but differentiated responsibility demanded by the right to health, and the FfD3, while identifying the diversity of potential contributors to development, not only did not offer a governance mechanism for ensuring they deliver, it specifically rejected it during the negotiations. (Global Policy Watch 2015)

\section{The management of externalities}

Frenk and Moon identify certain functions that contain the negative impact of decisions made by one state - or transnational body - on others. They argue for deployment of instruments such as surveillance systems, coordination mechanisms and information channels to respond to international risks to health. Examples include the global alert system for infectious disease, tsunami warning systems, and monitoring of radioactivityin the atmosphere to detect nuclear power plant accidents. The Lancet-University of Oslo Commission on global governance for health came to the conclusion that there are systemic global governance dysfunctions, undermining the management of externalities that impact health. The commission has identified democratic deficits, weak accountability mechanisms and poor transparency, institutional inertia, missing institutions, and an inadequate policy space for health, as key reasons why it is so difficult to manage externalities, or so the called "global bads" for health at an international level. (Ole Petter Ottersen et al. 2014)

In most cases where there is a severe threat to health arising from direct transnational developments, such as epidemic disease, there will be consensus among the states concerned about the action to be taken. However, this will not always be the case. Examples include hesitancy in notifying outbreaks of infectious disease because of concerns about the impact on trade or tourism, with the former a factor in the delay in recognizing the West African Ebola outbreak, cross-border movement of refugees fleeing conflict, as in Syria, or activities that restrict or contaminate cross-border water supplies.

Were these issues to arise within a state or at least one with functioning institutions, measures would be taken to enforce policies to address the fundamental problems. The scope to do so at an international level is constrained by the doctrine of state sovereignty. The revised IHR permit the wHO to draw on evidence from sources other than national governments when a disease outbreak is suspected.

However, beyond the changes to the IHR, developments in global cooperation have either been of little or no help in advancing the right to health or have actually undermined it. With many armed conflicts involving countries linked to, or protected by, a permanent member of the Security Council, action is frequently vetoed. (Hale 
et al. 2013a) International trade agreements place little, if any, weight on health considerations, tending to favor the powerful, which includes many corporations producing health-damaging products. Incorporation of health considerations is often cosmetic, such as the restriction on tobacco companies taking certain actions against states included in the Trans-Pacific Partnership, while leaving open the possibility of associations of tobacco producers, in effect front organizations for the tobacco companies, to do so. ${ }^{24}$

Thus, of the four functions, the institutional arrangements necessary to achieve the right to health seem weakest here.

\section{Does Global Health Governance in the SDGs Satisfy the Right to Health?}

The advent of economic globalization, in particular, has meant that some states and other global actors exert considerable influence on the realization of economic, social and cultural rights across the world. The Maastricht Principles on the Extraterritorial Obligations of States in the Area of Economic, Social and Cultural Rights clarify the legal principles for states to respect, protect, and fulfill human rights both within their domestic territories and outside their national borders. (Hammonds et al. 2012) In theory, the existing human rights legislation would enable the principles and basis for the global governance of health beyond the premise of the state. In practice, there is a governance gap between the human rights framework and practices in global health and development policies. This gap can be explained by the political determinants of health that shape the governance of these global policies (Tobacco Tactics, 2016).

The central question for this paper was: does the SDG agenda overcome that gap? Does the SDG agenda entail new or improved global health governance that satisfies the demands of the Right to Health? The answer is, unfortunately, negative. In each of the four functions of global health governance (according to Frenk and Moon), the SDG health agenda undercuts the Right to Health. Firstly, the stewardship function of global health governance is not addressed in the SDGs. Secondly, the GPGH that are included in the SDGs are insufficient. Beyond domestic legislation, there is no clear allocation of the responsibility to produce those global public goods. Thirdly, the mobilization of global solidarity merely includes the long-existing promise of High-Income Countries to spend $0.7 \%$ of their Gross National Income on Official Development Assistance complemented by a shifting focus on trade investments and domestic financing. Lastly, the management of externalities that impact health is hardly considered in the SDGS. All in all, the SDG agenda does not alter let alone improve global health governance.

24 Tobacco Tactics. Front Groups. (2016) http://www.tobaccotactics.org/index. php/Front_Groups. 
This assessment of relative neglect of human rights in the SDG health target is also consistent with a report on the World Bank, a major institution promoting the UHC target, by the UN Special rapporteur on extreme poverty and human rights. This rapport concludes that "the existing approach taken by the World Bank to human rights is incoherent, counterproductive and unsustainable. For most purposes, the World Bank is a human rights-free zone". ${ }^{25}$ The implementation of the SDGs will depend on the eventual realization of the financing framework agreed at the FfD3. It attributes a significant role for the private sector in development, without providing any mechanisms by which corporations can be held accountable. (Kvangraven 2015)

On the other hand, it has been argued that the SDGs do not depart from the discourse of accountability through enumeration established in the MDGs, but rather intensify it. The number of targets has increased from 21 to 169 and the indicators are likely to proliferate accordingly. Even richer countries would struggle with the data collection. The SDGS could have an epistemic, communicative and coordinating role but to truly play a constructive role in global development it might be wise to focus on the 17 higher-level goals, rather than the 169 targets. It might open up innovation, flexibility, and fuller democratic accountability. (Ooms et al. 2014) This resonates with Kickbusch's call for "a concept of global public health in the SDGs context which is democratic and ecological rather than utilitarian”. (Kickbusch 2016)

Finally, legal scholars have suggested that current representations of the right to health in the SDGs are insufficient and superficial, because they do not explicitly link SDG commitments or right to health discourse to binding treaty obligations for duty-bearing nation states or entitlements by people, whether legal citizens or undocumented migrants. (Carmel Williams and Blaiklock 2016) If global health policy is to meaningfully contribute to the realization of the right to health and to rights-based global health governance then future iterations of global health policy must bridge this gap. This includes scholarship and policy debate on the structure, politics, and agency to overcome existing global health injustices. (Benatar 2016)

25 Report of the Special Rapporteur on extreme poverty and human rights. UNGAA/70/27. Augustus (2015) http://www.un.org/en/ga/search/view_doc. asp?symbol=A/70/274\#sthash. oPzhm92l.dpuf. 


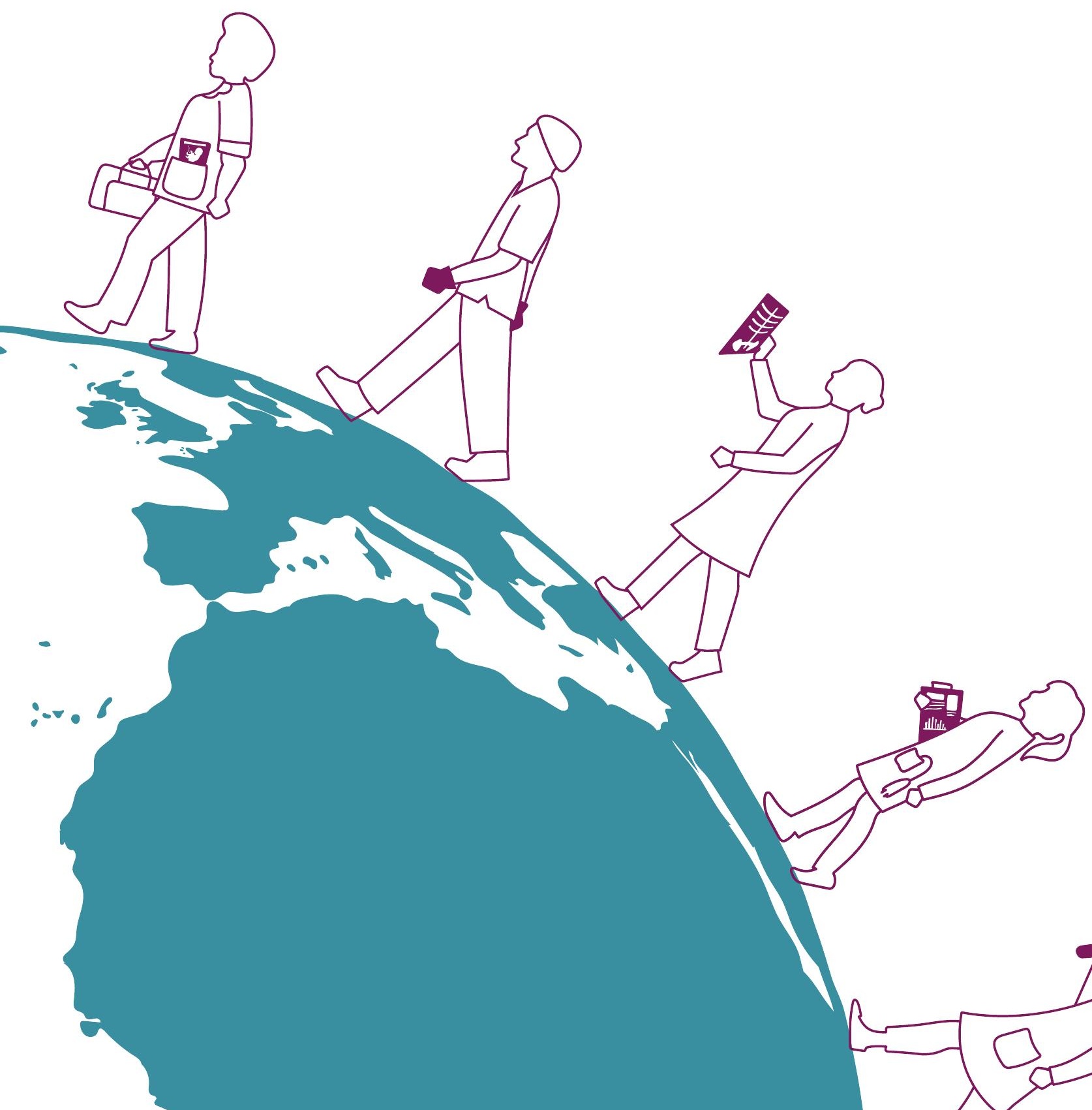




\section{2}

\section{Global Health in the Anthropocene: Moving Beyond Resilience and Capitalism}

van de Pas, R. (2017).

Global health in the anthropocene: moving beyond resilience and capitalism: comment on "health promotion in an age of normative equity and rampant inequality". International journal of health policy and management, 6(8), 481- 486.

doi:10.15171/ijhpm.2016.151 


\begin{abstract}
There has been much reflection on the need for a new understanding of global health and the urgency of a paradigm shift to address global health issues. A crucial question is whether this is still possible in current modes of global governance based on capitalist values. Four reflections are provided. (1) Ecological-centered values must become central in any future global health framework. (2) The objectives of 'sustainability' and 'economic growth' present a profound contradiction. (3) The resilience discourse maintains a gridlock in the functioning of the global health system. (4) The legitimacy of multi-stakeholder governance arrangements in global health requires urgent attention. A dual track approach is suggested. It must be aimed to transform capitalism into something better for global health while in parallel there is an urgent need to imagine a future and pathways to a different world order rooted in the principles of social justice, protecting the commons and a central role for the preservation of ecology.
\end{abstract}


Ronald Labonté's editorial on "Health Promotion in an Age of Normative Equity and Rampant Inequality" is part of an important ongoing debate on the future of global governance of health. (Labonté 2016) It is a debate about the fundamentals of global health challenges, more specifically on whether and why there is currently a pervasive sense of crisis in the numerous realms of the global health domain. There is much discussion about possible pathways and the governance mechanisms required to address "wicked" problems such as climate change, environmental degradation, increased income inequality, the obesity epidemic, antimicrobial resistance (AMR) and the largest human refugee streams since World War II. There has been much reflection already in this journal, elsewhere and since over a decade on the need for a new understanding of global health, the urgency of a paradigm shift to address global health issues that display a deep connection between health (and other) sectors, and the need for thorough reform of and investment in the international organizations mandated to address global health problems, notably the World Health Organization (WHO). (Bakker and Gill 2011; Baudot 2001; Benatar 1998; Benatar et al. 2003; Kent Buse and Hawkes 2015; Lawrence O Gostin et al. 2015; Harmer and Buse 2014; Kickbusch 2016) This is all punctuated with a growing understanding that global health policies are shaped by political agendas, powerful interests and inter-linked transnational networks of agencies and structures sharing like-minded norms and worldviews. (Benatar 2016; Gill and Benatar 2016a; Ole Petter Ottersen et al. 2014; Shiffman 2014) After elaborating on why and how to arrive at a number of Sustainable Development Goal (SDG) priority goals for health, and in some cases modifying them, a crucial question is posed by Labonte in the concluding parts of the paper: "How can we tame capitalism and the predatory market logic to support human equity and (now) a livable planet? Or, if it cannot be tamed, how might capitalism be transformed into something better fit for human social and ecological survival into a $21^{\text {st }}$ century?"(Labonté 2016)

\section{Different Paradigms}

Two different paradigms are proposed here. The latter is inspired by the transformative thinking and holistic values behind the SDGs but remains based on the Western development model as initiated after World War II, then institutionalized via the establishment of the United Nations (UN) and the Bretton Woods institutions (International Monetary Fund and World Bank) and later the Organization for Economic Cooperation and Development (OECD). This model follows the principles of economic growth (implying an expansion of (im)material extraction, production, and consumption) based on capitalism, free trade, democratization, good governance and the rule of law via cooperation between sovereign nation states. (Pronk 2015) Labonté labels this approach appropriately as "a gigantic global version of Franklin Roosevelt's New Deal," (Labonté 2016) basically a Neo-Keynesian 
investment model in green growth, decent employment, social protection and provision of public services and promoted, among others, by Nobel prize-winning economists such as Paul Krugman and Joseph Stiglitz who typically advocate for fiscal expansion to foster demand in the economy. Much of the thinking behind Official Development Assistance (ODA) has been (and still is) based on providing-via bilateral and multilateral channels-some form of redistribution and leveraging investment, loans, and capacity to LMICs so that they can 'grow out of poverty,' with regulation and norms to secure public goods and advance environmental and labor rights. The hope is that eventually, a (more) democratic organization of society with respect for basic human rights follows. The SDGs follow this path, by and large: the idea is to continue, albeit in a more inclusive, deepened and universal way, the trajectory of the poverty reduction objectives of the Millennium Development Goals (MDGs), but now combined with the ecological and sustainable development consensus as outlined originally in the Rio Declaration on Environment and Development. (United Nations Environment Programme 1992) The Ottawa Charter on Health Promotion and later also the work of the Commission on Social Determinants of Health, despite their progressive approach and demand for a reform of the dominant global economic model, both tend to advocate for change within a framework of incrementalism and inspired by a worldview that the current multilateral system of sovereign states, via balanced diplomacy, international agreements, foreign aid and policy coherence for development will eventually be able to protect the environment, secure public goods and safeguard peace and stability. As Labonté mentions, a glass-half-full galvanizes more than one half-empty, and he seems more optimistic about the potential of the SDGs than about the COP21 Paris Agreement. In any case, there is an immediate requirement to analyze the political feasibility of filling the SDG glass sooner rather than later: what can really be expected from the SDG framework as a 'politics of the improbable,' or is instead a real paradigm shift required to tame capitalism and the predatory market logic? Observing the current political directions in major G20 economies, with global governance for public goods and international power relations being multi-polarized and gridlocked, there is even much to argue that the glass is more than half-empty. There is a sense of urgency required in imagining and constructing alternative policy pathways for just and equitable globalization for global health. I provide four reflections to complement (rather than contradict) the arguments outlined in the paper.

\section{Ecology Becoming a Central Value in Global Health}

The former (paradigm) touches upon the centrality of ecological - centered values in any future global governance of health framework. Labonté refers to anthropogenic depravation, unequal ecological footprints, the promise of the fossil-fuel divestment movement, the 50 year-history of environmental critique as well as the importance 
of sustainable consumption and production patterns (while re-labeling them as the need to "consume sustainably"). Nevertheless, the focus remains in his approach, understandably, on the social and economic goals, for pragmatic reasons it appears. I would argue, instead, that the planetary ecology is so fundamentally jeopardized by the current global economic system that it must become a cross-cutting, if not central concern for all those working in global health, sooner rather than later. (Steffen et al. 2015; Turner 2014) A consistent, coherent understanding of global health must be developed that integrates social and ecological objectives. Gill and Benatar note that an "alternative paradigm of ecologically health ethics is sorely needed," one that is premised upon global solidarity and the "development of sustainability."(Gill and Benatar 2016b) Global health must be properly understood as an eco-centric concept embracing the idea of a healthy people on a healthy planet, recognizing the interconnectedness of life forms and human wellbeing as well as inspired by a deep sense of responsibility and respect for our "mother earth" and future generations. (Benatar et al. 2003) Inspiration and a moral frame can be found, for example, in the 'Laudatio Si' encyclical letter by Pope Francis,(Pope Francis 2015) but also in the Earth Charter. (Earth Charter Initiative 2015) Anthony J. McMichael argued for a 'sustainability transition' (McMichael et al. 2000) and the planetary health manifesto published two years ago stressed, rightly: "Planetary health is an attitude towards life and a philosophy for living. It emphasizes people, not diseases, and equity, not the creation of unjust societies...We need a new vision of cooperative and democratic action at all levels of society and a new principle of planetism and wellbeing for every person on this Earth."(Horton et al. 2014) In short, if this eco-centric approach is taken seriously we need to connect this concept with more 'traditional' global health objectives such as enhancing universal health coverage, reducing health inequalities, improving nutrition and access to essential medicines. If coherent, this would imply a shift from mere analysis and action on improving human and community health to a more inclusive consideration of the environmental ecosystem they are embedded in. For instance, this would shift the debate on how to deal with AMR away from the current focus on R\&D of new medicines to more attention for the understanding and adaptation of the ecological context that contributes to AMR in the first place. (McFarlane 2015)

\section{Fundamental Contradictions}

A second reflection relates to the priority SDG goals. SDG 17, on 'revitalizing the global partnership for sustainable development,' (United Nations 2015b) should be a priority to let the SDGs materialize. In essence, though, SDG 17 reveals much about the dominant political agenda lurking behind the SDGs, in spite of the transformative vision and lofty words in most of the other goals. While these are more universal, the indicators of SDG 17 still distinguish sharply between 'developed' and 'developing' 
countries. There is a lot of talk on 'nudging' countries into action and partnership with a focus on domestic resource mobilization in developing countries. In addition, "action is needed to mobilize, redirect and unlock the transformative power of trillions of dollars of private resources...long-term investments, including foreign direct investment, are needed in critical sectors, especially in developing countries."(Earth Charter Initiative 2015) In other words, the framing of this global partnership, combined with the 'blended financing' model of assistance, investment, and innovation as propagated by the Addis Ababa Action Agenda on financing of development (United Nations 2015a) makes it evident that there is still a strong belief in the harnessing power of economic growth without really acknowledging the public 'bads' and the social and environmental crises it has gotten the globe into in the first place, certainly in the last decades. Although there are many believers in 'green growth' nowadays, the objectives of 'sustainability' and 'economic growth' present a profound contradiction. (Hickel 2015b) SDG 17 does not aim for sharing the responsibility between countries by mitigating the historic human rights abuses (Pogge 2005a) that are at the root of stark differences between high- and low-income countries, and the unforeseen, but very serious (from a health perspective) sideeffects of industrialization for the integrity of the biosphere. SDG 17 does nothing fundamental to counteract the inherent and worsening instability of the current global economic system. The SDGs "offer to tinker with the global economic system in a well-meaning bid to make it all seem a bit less violent.” (Hickel 2015b) According to Gill 'global governance' is not just an analytical category but simultaneously an epistemological and strategic political project. Global health governance and the global partnership for sustainable development can be regarded as being part of a wider, though eroding, hegemonic project serving the outlook and interests of the most powerful states and affiliated actors. According to him, there is an organic crisis of global governance that raises fundamental questions about the legitimacy, ethical content and current forms of global leadership. In this sense-world order and global governance can be seen as an imperial system that is predicated upon the maintenance of a fossil-fuel intensive 'market-civilization' and the delay of an unavoidable energy revolution as it would be accompanied with inherent power shifts. (Gill 2015) A similar reflection was made by Naomi Klein in the 2016 Edward W. Said lecture on the ongoing violence of "othering" in a warming world. "Climate crisis must be seen in the context of austerity and privatization, of colonialism and militarism, and of the various systems of othering needed to sustain them all. The connections and intersections between them are glaring, and yet so often resistance to them is highly compartmentalized." (Klein 2016) Global health can be interrogated in the same way: in many instances, the 'othering' is perpetuated via framing it as 'just' a problem of developing countries with scarce resources and poor governance in a context of fragility combined with a limited awareness by communities of their health situation and lack of access to the innovative wonders 
of modern medicine. Global power structures that maintain inhumane health situations, such as those that became evident during the 2014-2015 Ebola outbreak in West-Africa, remain neglected. (Benatar 2015; Nunes 2016; Schrecker 2012) In our times of 'deep' economic globalization based on deregulated finance and free trade (plus the inherent democratic deficits), there is only a marginal policy space for nation states who find themselves in a fiscal race to the bottom to develop progressive social and ecological policies. Admittedly, there is 'a rise of the rest' and nation states and emerging economies, like the BRICS (Brazil, Russia, India, China, South Africa), have chosen alternative development modalities. (Kickbusch 2016) However, in our (now) multipolar world, these powers favor new financial institutions, known as the "new non-Western financial model," over-investing in the leadership, finance, and strategic directions of existing global health governance institutions such as the wHO, the United Nations Programme on HIV/AIDS (UNAIDS) or the Global Fund. (Kent Buse and Hawkes 2015) Political scholars have come to the conclusion that there is a gridlock in global governance domains, (Hale et al. 2013a) although there is debate whether this also applies to global health. (The Graduate Institute Geneva 2016) Studies from the ecological field clearly indicate the instability and limits of the current carbon-constrained capitalist growth model and the planetary boundaries. (Benatar 2016; Ole Petter Ottersen et al. 2014) In short, the analysis above provides a sobering view on (the expectations for) the financing to be generated via the global partnership on development. The glass will probably only remain half-full or, in these times of increasing nationalism and a backlash against globalization, might even be emptied further. Consequently, if the world remains within this capitalist model, even of a more "sustainable" and "inclusive" kind, grand global health claims such as "a world converging within a generation" (Jamison et al. 2013) or the prediction that the current trend of economic growth will continue and provide the fiscal space in Low- and Middle-Income Countries (LMICs) to employ 18 million extra health workers required to attain the health objectives of the SDGs by 2030 (World Health Organization 2016b) remain a gamble.

\section{The Status Quo of Resilience and Multi-stakeholder Governance}

The third and fourth reflection follow the thinking above that the sDGs might (only) be locked in the status quo of global governance rather than display the transformative shift they are usually associated with. Reflections on resilience and multi-stakeholder governance are dealt with on their own merits in longer debates and papers (Harman and Williams 2013; Resilience Journal) so they are only touched upon briefly here. In his article, Labonté alludes to the distraction of the resilience discourse and I can't agree more with him. Although resilience capabilities might be one of more desirable outcomes of health systems strengthening (Kutzin and Sparkes 2016) the normative thinking behind much of the current resilience 
discourse is that crises are permanent and that individuals, thus, have to be permanently prepared for the worst. Evans and Reid note: "The real tragedy for us is the way the (resilience) doctrine forces us to become active participants in our own de-politicization... it even demands a certain exposure to the threat before its occurrence so that we can be better prepared. Resilience as such appears to be a form of immunization." (Brad Evans and Reid 2015) By internalizing resilience as the main principle of dealing with insecurity, it becomes part of self-policing. Neocleous concludes "In so doing resilience shapes our political imagination and thereby cuts off alternate political possibilities." (Neocleous 2015) This includes cutting the moral imagination (the ability to imagine oneself in the shoes of others) that can enable to alter one's outlook and actions significantly. (Benatar 2005) The Ebola outbreak in Western Africa catalyzed further development of the Global Health Security Agenda, a partnership representing governments, academia and the private sector, built on the value of resilience and the notion that "Our connectedness... poses serious challenges with implications for our health security and for the stability and security of our populations." (Pope et al. 2016) The very valid question remains whose interests and whose security are predominantly being served by this agenda, and whether alternative models of overcoming infectious diseases epidemics can be developed. (Rushton 2011) Multi-stakeholder governance is mentioned by Labonté as one of the core contradictions of the sDGs. Indeed, without enforced regulation for the public good, this sort of governance might continue to be a smokescreen for legitimizing the powerful actors and interests that contributed most to the current economic and ecological crises. While in general there is a need to strengthen forms of deliberative democracy beyond the nation state, (Habermas 1996) including in institutions dealing with global health, a lot needs to improve on the output legitimacy (accountability, transparency effectiveness) and certainly input legitimacy (deliberation and representation) of global health and sustainable development regimes. (Remco Van de Pas and Van Schaik 2014) Research on the development of the health SDG goal has indicated limited participation of local communities in setting priorities for this goal and the governance gap between the global policy-makers and the 'target groups.' (Siddiqui et al. 2014) Proper regulation, the management of conflicts of interest and a strong democratic framework to govern global health programs all require close attention in the implementation of the SDGs. This should not merely be about the international organizations itself and their engagement with corporate actors, but also touch upon the politics and agency of philanthropic organizations, academia, and civil society. (Kent Buse and Hawkes 2016; Harman 2016; Leschhorn et al. 2016; Sénit et al. 2017) 


\section{Moving Forward}

In conclusion, I would argue, somewhat similarly, for a 'dual track' approach as formulated by Labonté. We have to continue with our aim to gradually transform capitalism into something better while in parallel we should be well aware that we need to move beyond resilience and capitalism fast, and thus, imagine a future and pathways to a different world order rooted in the principles of social justice and protecting the commons with a central role for the preservation of ecology. Despite my reservations described above, multiple health crises and the cosmopolitan window of opportunity they create (Kickbusch and Reddy 2015) could perhaps trigger a momentum within the SDG framework to have more global public goods for health universally (co)financed, such as basic public health functions and universal health coverage. It must, however, be noted that current representations of the right to health in the SDGs are insufficient and superficial, because they do not explicitly link commitments or right to health discourse to binding treaty obligations for dutybearing nation states or entitlements by people. (Remco van de Pas et al. 2017a) If the crises become deep enough there will be a stronger push for global redistribution mechanisms like an international tax regime or the need to regulate the harms of our overheated consumerist societies. (Atkinson 2015a) More importantly, we should allow for moral and political imagination and conceptualize alternative views of organizing societies. Recent publications on the 'Politics for the Anthropocene' (Purdy 2015) and 'Realistic Cosmopolitanism' (Beck 2006a) hint towards the inherent shared responsibility required to govern civilization, the environment and global risks. Thinking along the lines of the End of capitalism (Mason 2015) the Basic Income Earth Network (Basic Income Earth Network 2016) as well as the Degrowth and Divest movement, (Research and Degrowth 2016) Indigenous principles of 'Buen vivir' (Balch 2013) and citizens reclaiming the common goods (Mestrum 2016) all provide elements of hope. The global health community should hence not only pursue this important debate in academic journals or at global policy meetings but participate actively in societal movements and debates to help drive a real (and clearly much needed) paradigm shift. This trajectory is deeply political and risky. In the words of Eduardo Galeano:

"I advance two steps, it goes two steps backward. I take ten steps and the horizon moves ten steps forward. No matter how far I walk, I will never reach it. What is the use of utopia? That's its use: to help us walk." (Galeano 1993) Yet, we owe it to the next generations. 


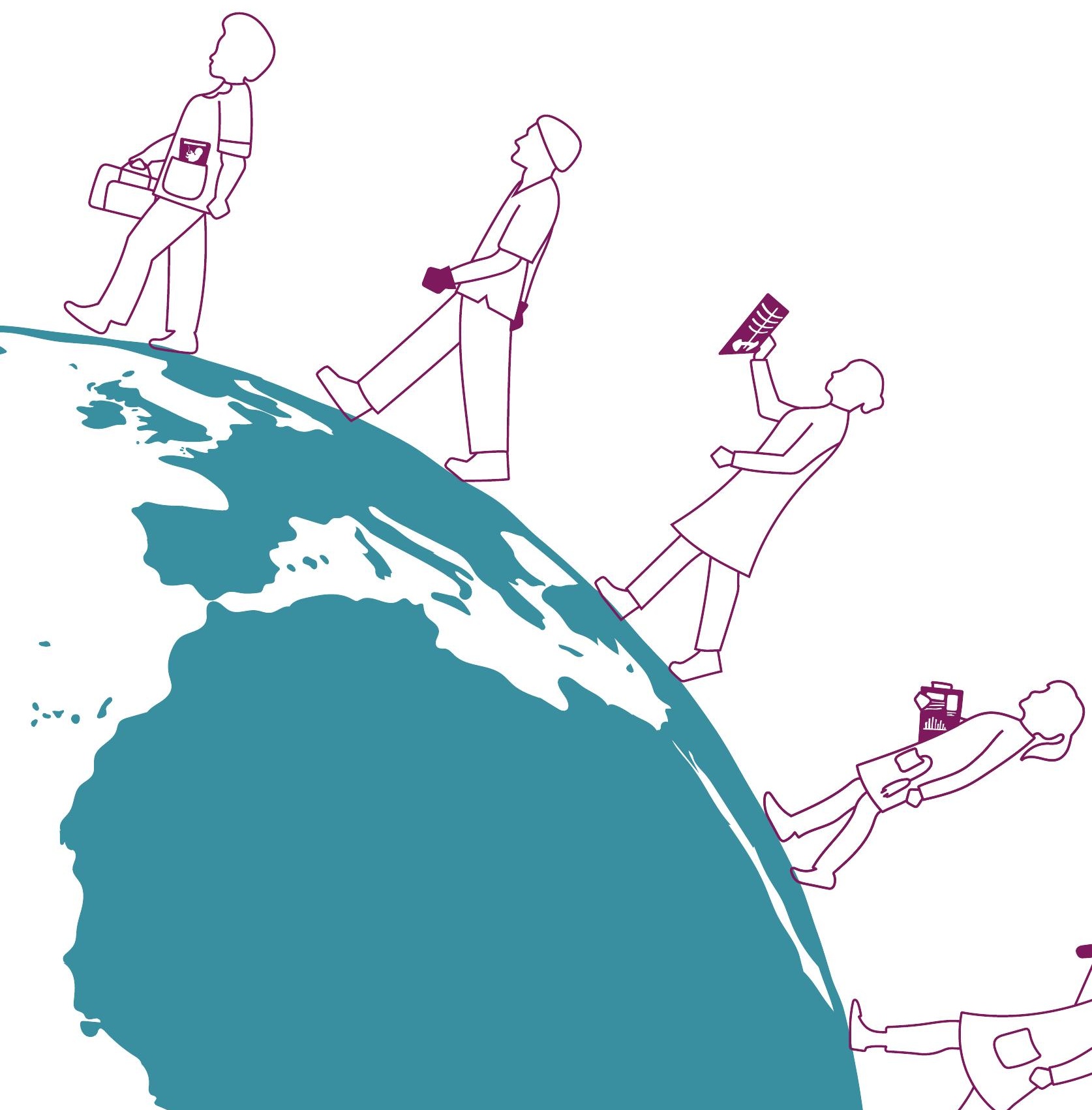




\section{3}

\section{Framing the Health Workforce Agenda Beyond Economic Growth}

van de Pas, R., Mans, L., Bemelmans, M., \& Krumeich, A. (2018).

Framing the health workforce agenda beyond economic growth.

International journal of health policy and management, 7(8). 678-682.

doi:10.15171/ijhpm.2018.45 


\begin{abstract}
The fourth Global Forum on Human Resources for Health (HRH) was held in Ireland in November 2017. Its Dublin declaration mentions that strategic investments in the health workforce could contribute to sustainable and inclusive growth and are an imperative to shared prosperity. What is remarkable about the investment frame for health workforce development is that there is little debate about the type of economic development to be pursued. This article provides three cautionary considerations and argues that, in the longer term, a perspective beyond the dominant economic frame is required to further equitable development of the global health workforce. The first argument includes the notion that the growth that is triggered may not be as inclusive as proponents say it is. Secondly, there are considerable questions on the possibility of expanding fiscal space in low-income countries for public goods such as health services and the sustainability of the resulting economic growth. Thirdly, there is a growing consideration that economic growth solely expressed as increasing gross domestic product (GDP) might have intrinsic problems in advancing sustainable development outcomes. Economic development goals are a useful approach to guiding health workforce policies and health employment but this depends very much on the context. Alternative development models and policy options, such as a Job Guarantee scheme, need to be assessed, deliberated and tested. This would meet considerable political challenges but a narrow single story and frame of economic development is to be rejected.
\end{abstract}




\section{Health Employment and Economic Growth}

The Fourth Global Forum on Human Resources for Health (HRH), held in Ireland in November 2017, had the aim of furthering a bold economic case for investing in the health and social workforce, and intensifying inter-sectoral coordination. The Dublin declaration builds on the report of the United Nations Secretary-General's High-level Commission on Health Employment and Economic Growth (UNHEEG) and its benefits across the Sustainable Development Goals (SDGs). (World Health Organization 2016e; 2017b) The declaration also mentions that strategic investments in the health workforce (HWF) could contribute to sustainable and inclusive growth and are an imperative to shared prosperity. Over the last few years, the World Health Organization (WHO) has made economic growth the dominant, but not exclusive, frame for HWF development. In its publications, the WHO explicitly mentions the need to frame the HWF agenda in a way that generates political will for HWF development. (Cometto et al. 2013; World Health Organization 2017c) Social theory provides the insight that similar issues can be framed in different ways by different actors. (Koon et al. 2016) Framing analysis and its relevance for global health governance and policy-making has become more prominent. (McNeill and Ottersen 2015) The framing of global health challenges has important power implications for the determination of policies and action, and therefore on the solutions that are proposed for dealing with a problem. (Koon et al. 2016) Nearly a century ago, in his thinking on 'linguistic hegemony' or 'cultural hegemony,' Gramsci already provided the analysis that "In a vital sense language is politics, for it affects the way people think about power." (Boothman 2017) Lipmann, around the same time, introduced the term 'manufacturing consent' as a possibility to shape and manipulate the public opinion in democratic societies. (Lippmann 1922)

What is remarkable about the investment case as a frame for HWF development is that there is little debate about the type of economic development to be pursued. Rather, 'inclusive growth' as the outcome of HWF investments is considered a given. This article provides three cautionary considerations of this principle and argues that in the longer term a perspective beyond the dominant economic frame is required to further equitable development of the global HWF.

\section{The Health Labor Market and Fiscal Space}

The WHO has made labor market analysis the central framework for assessing HWF requirements both at national and global levels. It uses supply-, need- and demand-models to provide scenarios on how the workforce will likely develop over the following years. (Cometto et al. 2017) Over the years, the World Bank (WB) has become more engaged in HWF development. Guided by its focus on employability, 
poverty eradication and shared prosperity the wB has recognized that the health services sector provides a considerable economic growth potential contrary to 'traditional' industrial and extractive sectors. The wB has conceptually paved the way to assess health services from a labor market and fiscal perspective as a strategy for economic development in Low- and Middle-Income Countries (LMICs). (Soucat et al. 2013; Vujicic et al. 2009) Both in the domain of HWF financing as well as health financing strategies such as Universal Health Coverage (UHC) the wB has started to collaborate closely with the wHO. This does not only include cooperation and exchange at the technical level but also joint leadership and global commitment for UHC. (Ghebreyesus 2018)

The labor market framework provides the insight that in LMICs a major problem is not merely the lack of an available skilled HWF but also the insufficient (economic) demand to finance health sector employment, thereby emphasizing the need to invest in jobs. Evidence also suggests that health labor markets are not intrinsically wellfunctioning. In order to reach a 'market-cleaning equilibrium' health labor markets require regulatory or institutional interventions to achieve socially desirable and economically efficient outcomes (e.g., universal access to a health worker's services via incentives to having the health worker retained for employment in rural areas). (McPake et al. 2013) The wHO provides a conceptual overview of how investing in health systems can lead, via six pathways, to economic growth. The authors hereby mention that the concepts 'efficiency' and 'growth' are interlinked and provide two arguments for why growth is relevant for societies; first, by "producing more benefits, in terms of income, consumption, investment, production, and other forms of (mainly) marketvalued benefits." Secondly, growth requires government action to correct market failures (inefficiency) such as negative externalities and to provide public goods (e.g., education and health care). (Lauer et al. 2017b) The question is then, how to pay the wage bill for the additional HWF required in order to attain the SDGs, and how to secure fiscal (public) and financial (public and private) space? An analysis shows that conditional on "current trends of economic development and population growth" there are challenges to securing the wage bill in a (small) number of countries (optimistically, as few as 4-16 countries; less optimistically, as many as 69 countries). The authors conclude "that the number of countries requiring sustained development assistance for wage bills from donor nations is likely to be limited, possibly to as few as 20-30 countries or even fewer." (Lauer et al. 2017a)

In the analysis above the underlying notion is that economic growth, properly regulated by public authorities, is required to meet the Sustainable Development Agenda and its health-related goals. In essence, the SDGs are "one gigantic global green version of Roosevelt's New Deal agenda" to advance socio-economic and ecological goals. (Labonté 2016) However, Labonté notes that there is a contradiction at the 
heart of the SDGs that builds on an implicit assumption that the same economic rules that have created an increasingly unequal and unsustainable world can somehow engineer the reverse. (Labonté 2016)

\section{Alternative Analyses on Economic Development Needs}

Three critical remarks can be made about the concept of (inclusive) economic growth being the desirable outcome of investments in health employment. The first considers the notion that the growth that is triggered may not be as inclusive as proponents say it is. While there is evidence that regulated economic growth might improve equitable access to health services, this attribution is not self-evident for health inequalities in principle. A range of countries in several regions of the world have used economic growth to enhance access to health professionals and sustainable health employment. (Dal Poz et al. 2015; Dussault et al. 2016; Remco van de Pas et al. 2017b) At the same time income and wealth inequalities in many countries, although not necessarily between countries, have been growing over the last decades. Social policies as a way of public redistribution, such as social protection as indicated in one of the pathways by the wHO, are a possible instrument for reducing income inequalities which would, in turn, lead to a reduction in health inequalities. (Jutz 2015) However, this is not sufficient. Milanovic, by pointing out the growing disconnect between labor and capital, analyzes this 'new capitalism' as a major reason for the growth in global inequalities and argues for long-term equalization of capital ownership and education. (Milanovic 2016b) Rather than focusing on economic growth via mere investment in labor (such as in the HWF), a reduction of income inequalities via high-inheritance taxes, corporate tax policies and broader ownership of assets (by the poor and middle-class), as well as equalizing meaningful access to education, are policy recommendations for effectively reducing inequalities. The assets that would become available could then be reinvested in health employment and building a sustainable workforce. Milanovic makes the case that economic growth is still needed in poor countries. However, to make it ecologically sustainable, restraints on growth should be imposed on the rich countries. (Milanovic 2016b) SDG goal 10, on reducing global inequality, is disappointing as only target 10.1 has as its aim to "progressively achieve income growth for the bottom $40 \%$ of the population." Big drivers of poverty and global inequalities accumulated through private wealth accumulation are neglected by the sDGs and remain unaccounted for. (Hickel 2015a)

Secondly, there are considerable questions by scholars on the possibility of expanding fiscal space for public goods in low-income countries and the sustainability of the resulting economic growth. Assessing fiscal space for financing health systems has gained momentum by health economists, but their main focus has been on increasing 
domestic revenues in line with recommendations by the Addis Ababa Action Agenda on financing for development. (Barroy et al. 2018) Rodrik, however, has clarified the tension between national democratic decision space and global markets as the 'political trilemma of the world economy.' In this trilemma there are basically three options; restrict democracy in the interest of minimizing international transaction costs (e.g., labor wages); limit globalization in the hope of building democratic legitimacy at home; or to globalize democracy at the cost of national sovereignty. The 'trilemma' exists in the challenge that at most two out of these three options can function together. Too often the reality of sovereign nation states functioning in a hyperglobalized order is them being locked in a 'Golden Straitjacket.' (Rodrik 2011b) In this model, national, democratic, economic and fiscal policy space and its governance is inevitably restricted. The other possibly attractive options of limiting globalization by rethinking trade and investment agreements in order to expand democratic decision-making or to globalize democratic governance along with markets have so far had too much resistance from both old and new major state powers. (Rodrik 2011b) The limitations of fiscal flexibility are outlined in an analysis on the impact of the International Monetary Fund (IMF) conditionality (1995-2014) on government health expenditure in 16 African nations. Despite the rhetoric that in recent years the IMF has started to promote social protection policies and health systems strengthening, the evidence reveals that, under direct IMF tutelage, these countries have had limited policy space and considerably underfunded their health systems. (Stubbs et al. 2017) For instance, in Malawi, 60\% of the wage bill for the required staff establishment to meet essential health services is not funded [Clinton Health Access Initiative \& Ministry of Health, Unpublished data, 2016]. Albeit health professional staff graduating in significant numbers, often with the scholarship support of donors, there has also been a freeze on the recruitment of staff. This has followed IMF recommendations to the government that a key priority in the short term is to restore macro-economic stability and that "an appropriately tight fiscal policy is needed." (International Monetary Fund 2015) Despite assumptions of continued economic growth, characterized by a divergence of paths between countries, Africa's economies have seen a slowdown over the last couple of years. (Russo et al. 2017) Health policies and its financing must incorporate the realities of non-linear economic growth and potential economic contraction. In the face of economic crisis, countercyclical measures should be brought in to mitigate its effects and provide social protection for low-income and vulnerable populations. (Russo et al. 2017) In times of economic volatility, rather than leaving the onus of health employment financing in LMIcs a sole domestic responsibility, it would be fairer to develop a coherent global framework for health financing based on shared responsibility principles. Such a framework is built on 4 principles (a global pact); domestic financing, joint financing of global public goods, external financing for national health systems, and a global agreement and accountability mechanism. (Trygve Ottersen et al. 2017) 
Thirdly, and truly paradigmatically different, is the slow but growing consideration that economic growth solely expressed as increasing gross domestic product (GDP) might have intrinsic problems in advancing sustainable development outcomes. Woodward has calculated that under current 'pro-poor' economic development models it would take over 200 years to attain the eradication of poverty (measured at USD 5 per person per day as poverty baseline). To do so, global GDP would have to increase to 175 times its present size. "There is simply no way this can be achieved without triggering truly catastrophic climate change." It basically implies that we should shift our attention from global economic growth to the (re-)distribution of the benefits of global production and consumption. (Woodward 2015) This principle is in essence also put forward by Raworth, who in her thinking on circular economics puts forward a model, the Doughnut, of social foundations and planetary boundaries (our 'ecological ceiling'). (K. Raworth 2017a) She urges us to move from being growth addicted to being growth agnostic, and argues that economies should become distributive by design. This implies that investments in public goods, such as health employment, would be decoupled from economic growth and be achieved by tax justice and wealth redistribution, as outlined by Milanovic above. However, he considers such policy reforms not (yet) politically feasible in current times. (Milanovic 2017) In the circular economy, health employees would ideally work for public, democratic, accountable institutions or member/employee-owned companies that would have a distributive enterprise design instead of a profit-oriented shareholders model. (K. Raworth 2017a) Stiglitz and Sen have put it very clearly; GDP is not a good measure of economic performance; it is not a good measure of well-being; it is a mismeasurement of life. (Stiglitz et al. 2010)

Furthermore, the Degrowth economic paradigm and its movement are slowly gaining momentum. It postulates that all countries have a common but differentiated responsibility to fulfilling basic development goals. This would imply that poor countries may grow their economies until at least 2025, while richer countries downscale production and consumption by around $6 \%$ per year. This would allow poorer countries to use up a disproportionate share of the global carbon budget for socio-ecological development, for example by investing in health employment. (Hickel 2017) The chairperson of the commission of the African Union has concurred as follows: "African Youth represent more than 60\% of the population in the continent. Without a heavy investment in this youth, its education, training, employment, and intellectual capacity...Africa does not have a future." (Hickel 2017) He then continues as follows; "The question of emigration, especially to Europe, arises in tragic terms. This is our common challenge. Our shared responsibilities here are excruciating; they challenge us in the depth of our consciences." (Faki Mahamat 2017) In line with this plea, Milanovic and Rodrik both argue for a new deal on labor mobility; making the case for international agreements on facilitating temporary work visa programs including for labor mobility in the health services. (Milanovic 2016b; Rodrik 2018) 


\section{The Relevance of Economic Growth and Other Useful Frames}

To be clear, we do not argue that the economic growth frame should be left unconsidered when reflecting on how to develop the HWF and generate investments for health employment. A health labor market and fiscal space assessment can help make the right policy choices. The global strategy on HRH asserts that domestic resources for $\mathrm{HRH}$ should be supported by appropriate macroeconomic policies at national and global levels and that, at least under certain circumstances, "countries will require overseas development assistance for a few more decades to ensure adequate fiscal space." (World Health Organization 2016g) Sustainable and inclusive economic growth in low-income countries is something to strive for. This is to be accompanied by progressive corporate tax policies, tackling illegitimate capital flight and closing down tax havens, as well as redistribution of the assets resulting from economic growth into social goods such as health services. Moreover, the gender balance of health employment is also of relevance. Women constitute $60-70 \%$ of the HWF in most countries. Targeted investment in this labor group would contribute to addressing gender inequality at the workplace, with potential impacts in the household and in society in general. (Langer et al. 2015)

Nevertheless, the WHO and other key actors in HWF policy must be encouraged to recognize, research, deliberate and test alternative frames, guiding HWF development, and the different corresponding political pathways to change. (McNeill and Ottersen 2015) When these actors claim 'inclusive economic growth' as the outcome of HWF investments, they do so referring to SDG 1 (poverty elimination), SDG 3 (good health and well-being), SDG 4 (quality education), SDG 5 (gender equality) and SDG 8 (decent work and economic growth). However, the social determinants of both human health and environmental degradation should not be neglected. (McCoy 2017) The security frame has often been invoked since the Ebola Outbreak of 2014-2015 in West-Africa. A skilled workforce is required to generate the capacities for global health security. (The Lancet - Editorial 2016) Other policy options have somehow been neglected in the HWF governance 'discourse.' For instance, the notion of the HWF being a requirement for delivering Global Public Goods for Health (GPGH) has not been mentioned by the UNHEEG report. Functioning health systems can be considered an 'access' good for GPGH and presents a strong case for the provision of free health services at the national level, and for external subsidies needed to achieve this. (Richard D Smith and Woodward 2003) Also, from a health equity perspective, values (frames) such as 'health and human development,' 'health as a human right' and 'health and global justice' are to be considered. (McNeill and Ottersen 2015) From a development angle, one could build on the health capability approach, and the implicit health systems and providers responsibility to pursuing this. (Ruger 2010) Although there is reference 
by the UNHEEG report on the International Labor Organization's recommendation 202 to the right to social protection, and gender equality, this is mostly applied to the social security rights of health workers themselves. (International Labour Organisation 2012) A decade ago more attention was given to the human Right to Health and how it contributes to HWF development. (Health Workforce Advocacy Initiative 2009)

\section{A Job Guarantee Scheme for the Health Services Sector}

Interestingly, more labor proposals are increasingly returning to a social policy framework that was popularized during and shortly after World War II; guaranteeing full employment as a strategy to realize macro-economic, redistributive and collective outcomes. (Klosse and Muysken 2016) The late Tony Atkinson, the godfather of inequality research, promoted a job guarantee scheme in his Magnus Opus; 'Inequality, What can be done?' (Atkinson 2015b) An elaborate proposal on the Job Guarantee, a public option for jobs, has recently been published. "It is a permanent, federally funded, and locally administered program that supplies voluntary employment opportunities on demand for all who are ready and willing to work at a living wage." (Tcherneva 2018) Future research is required to see if and how full employment schemes can be implemented and financed in the health care sector, assess its broader impact on socio-economic outcomes, and gauge the policy space that is possible in highincome counties as well as LMICs to pursue such social strategies.

Unfortunately, the human rights approach to health has largely been left out of the Sustainable Development Agenda. (Remco van de Pas et al. 2017a) A global justice (shared responsibility) approach to health systems development and health employment, within ecological limits, could be materialized by effectuating mechanisms such as a coherent global framework for health financing, a Job Guarantee scheme or applying Raworth's Doughnut model on circular economics to health systems development. (McCoy 2017; K. Raworth 2017a)

\section{Conclusion: Framing and Differentiating the Health Workforce Agenda}

In conclusion, economic development goals are a useful approach to guiding HWF policies and health employment but this depends very much on the context. It does call for sustainable and inclusive economic growth in LMICs, and Degrowth and delinking health employment from economic demand in countries beyond a certain income level. Low-income countries struggling to address health challenges still need sustained international support and targeted measures in order to address underlying inequities in the global HWF distribution. (Bemelmans and Philips 2017) This also requires the assessment, deliberation and testing of 
alternative development models and policy options, such as the Job Guarantee scheme. We realize that it would meet considerable political challenges but a narrow single story, a frame, of economic development is to be rejected. 'The future is fertile and rich with possibility; we need only have the courage to invent it.' (Woodward 2015) 


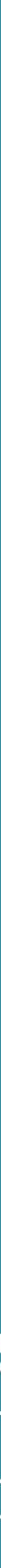


In this part, I will analyze and discuss the previous chapters based on the overall research question of this thesis: Can health workforce (HWF) policies rooted in a cosmopolitan outlook advance HWF development? The studies in the preceding chapters have researched the four governance questions relevant to HWF development. The four chapters and their themes form the structure for the first part of the discussion. The chapters follow an outline of the global health governance conceptualization along three political spaces. (Kickbusch and Szabo 2014) At the same time, these studies also take, implicitly or more directly, the 'political trilemma' (Rodrik 2011a) into consideration and provide an explanation as to why there are inherent 'global' political and economic tensions in developing the HWF and its related governance structures. The last chapter studies whether and how it is possible to expand universal global health policies, including for the HWF and provides for perspectives on whether there is space for a cosmopolitan orientation.

The methodological approach and constraints encountered in the studies will be then reviewed in the latter part of the discussion. The studies were conducted as part of a larger research and development cooperation program and funded via different means. This has impacted the scope and results of the studies. This thesis, which took more of a boundary-spanning approach to a global health challenge (Sheikh et al. 2016) had to build upon study approaches from different disciplines. This provides difficulties in reaching a comprehensive analytical conclusion. I have attempted to provide a reflection on the limitations and challenges encountered in integrating the different elements of this thesis by presenting an analysis on advancing global HWF development based on the cosmopolitan thinking and policy suggestions by Beck and other scholars. This part will also reflect on the overall framework of the 'Political Trilemma in the Governance of Global Health Workforce (GHW)' and whether there is a way forward beyond the current gridlock in global cooperation in relation to the HWF challenge. It will also explore whether the original framework has been an appropriate analytical lens to assess the Human Resources for Health (HRH) governance problematic or whether other approaches would be more relevant in dealing with this global health issue. The thesis will end with thinking on how to advance policy, research, and approaches towards a more cosmopolitan, transnational, consideration of HWF development. Lastly, an addendum has been added (annex 1) to this chapter valorizing the outcomes of this thesis in relation to policy development, implementation and furthering scientific debate. 


\subsection{DOMESTIC POLICY SPACE TO EXPAND AND REFORM THE HEALTH WORKFORCE}

Chapter 3 studied how national HRH policies have been pursued by governments and other actors. The question is whether governments in countries with HRH challenges do actually have the policy space to expand and reform the workforce? I refer to this as 'Governance for Global HRH' in the overall framework (Figure 1.3). I have studied this policy space by tracing health policy developments and health systems strengthening in Guinea in a post-Ebola epidemic setting. Secondly, I have traced the policy actions of 57 states and 27 other actors as a follow-up of the $3^{\text {rd }}$ global forum on HRH in Brazil, 2013. Both studies provide the insight that HWF policy development is not a linear process, but follows certain 'shocks'. These shocks might be initiated by external events or are made possible due to political and economic changes at the domestic level. In the case of Guinea, the need to develop a strong HWF and consecutively to develop a resilient health system was driven by both domestic as well as international concerns following the Ebola Virus Disease (EVD) outbreak in 2014. After such a political momentum a stable sociopolitical and economic situation is required for the government to create demand and expand its investments in the workforce. Several countries have been able to reform and invest in their workforce during the last decade. The tracing study indicates that such investments were possible in countries as diverse as Ethiopia, Ecuador, Indonesia, Sudan, Ghana, and the Republic of Moldova. It must be noted that these countries have seen considerable economic growth during a number of years and that political leadership, as well as institutional capacity, supported investment in the (public) health care sector. These investments were hence pro-cyclical. It implies that these investments took place when economies were in a high conjuncture phase. In the tracing study conducted there is no evidence of countries pursuing counter-cyclical investments (Keynesian economics) in the workforce during times of economic hardship. This indicates that HWF development is fragile and influenced by domestic politicy choices, political changes as well as economic cycles. For instance, international pressure for reform and domestic political pressure allowed for investment in the Guinean health system post-Ebola. The government increased its health expenditure to $8 \%$ of the national public budget. This has led to the recruitment of about 4,000 health workers to work in rural areas. Nevertheless, results from follow-up research indicate that there are serious concerns about sustainability of these investments and reforms. (Kolie et al. 2019) This is partly because international interest in the financing of the Guinean health systems has reduced following the waning of the health security threats. Three years after the end of the EVD outbreak, the main interest of international donors has been to finance community health workers (CHW) programs in rural areas ensuring early warning of, and rapid response to, emerging infectious diseases as well as the continuation of ongoing vaccination programs. At the same time, there 
has been, due to domestic political choices, an impasse in the financial reform and governance of the health system. With limited capacity and means to ensure that health personnel will be retained in the rural areas, there is a risk that many of them will return to the main urban centers. (Kolie et al. 2019) There has been much attention over the last few years for the need to strengthen global health security. This has, in the case of Guinea, not really led to a positive 'spillover' in relation to the development of a national health insurance system and strong health system. While this might change from country to country, there is no evidence that there is a true synergy between Universal Health Coverage (UHC), health systems strengthening (HSS), and global health security programs. (Ooms et al. 2017)

\subsubsection{International finance}

Both the Guinean case study and the comparative tracing study covered in chapter 3 indicate that there remains a tension in relation to a 'shared-responsibilitiesapproach' (a cosmopolitan outlook) in financing essential health care services in Low- and Middle-Income Countries (LMICs). Internationally, the development consensus focuses on the requirement by national governments to domestically finance their health system. (Montes 2016) Researchers have proposed a mixed target of a proportional indicator (5\% of gross domestic product; GDP) as well as an absolute target (USD 86/capita) as an appropriate benchmark for the financing of essential public health care services. Even if all countries reached the relative target of $5 \%$ of GDP, no low-income country (LIC; such as Guinea) and only $60 \%$ of LMICs would be spending above the absolute target of USD 86 per capita. This highlights the need for external support to secure the needed health care in countries which even at their 'maximum available resources' cannot meet this absolute target alone through domestic resources. (McIntyre et al. 2017) The same authors argue that even LICs have the possibility to increase fiscal space for investment in social services and health care jobs via progressive taxation measures and natural resources extraction. The ability of LMICs to successfully implement such strategies for increasing government revenue is in many ways dependent on supportive global action. This includes addressing tax competition and improving transparency in business activities, tax payments and payments to governments by extractive companies. (Meheus and McIntyre 2017) Such an approach could eventually be part of a coherent global framework, based on shared responsibilities, for health financing including for the HWF. Despite much focus on domestic financing, external health financing for health systems will remain critical for the years to come. (Ottersen et al. 2017) Economists from the World Health Organization (WHO) have estimated that USD 371 billion per year is needed to reach universal health system targets by 2030 in 67 LMICs. (Stenberg et al. 2017) Development Economist Jeffrey Sachs provides a simple and stark argument: "Because of a shortfall in ODA [Official Development Assistance] of a 
mere $0.1 \%$ of GDP by the rich countries, millions of people die unnecessarily and tragically in the low-income countries." (Sachs 2019) According to him, UHC must be a matter of global solidarity between the rich and the poor. Sachs proposes that "a 1\% net worth tax on billionaires could in principle fund both universal health coverage and universal education access in the low-income countries." Alternatively, he suggests a modest transfer from military funding to health care services in LICs. (Sachs 2019)

However, the study in Guinea and the countries studied in the comparative tracing study (majority of them LICS and LMICs) indicated that external funding is in vast majority being used to finance humanitarian aid and Global Public Goods for Health (GPGH), especially for health security objectives such as progressive immunization, surveillance, and the control of a limited number of infectious diseases. Regarding the workforce, most of the external funding is being used to pay for (temporary) CHW training and their salaries as part of international health programs. While some external funding is used to recruit formal health professionals such as doctors, midwives, and nurses, this is as a rule always off-budget and via temporary programs. The main thinking hereby is that external funding is not a fiscally sustainable approach to finance recurrent costs such as wage bills of public health workers. (Heller 2005) However, temporary contracting of health workers has its limits in developing a sustainable domestic workforce (a public worker or civil servant contingency). Despite governments often 'promising' to development partners that they will absorb the temporary workforce as civil servants upon finalization of contracts, this is often not respected due to fiscal constraints being set by the Ministry of Finance. (Wemos 2019)

\subsubsection{Economic reforms and fiscal space limitations}

The tracing study on HRH actions in chapter 3 indicates that several LMICs have chosen to invest domestically in the HWF and were able to (partly) reform the HRH sector. The 'straightjacket', as referred to in Rodrik's political trilemma, does allow some space for policy prioritization, including for the public health sector, as long as there is economic growth. How much space countries have and actually take differs though. Let us take for example Ethiopia and Rwanda, two LICs in Eastern Africa that have been hailed as examples for the rest of the continent. Both have invested considerably in the public health sector with Rwanda spending about $9 \%$ of its government expenditure on health. For Ethiopia, this is 6\%. In both countries GDP growth has doubled over the last few decades, which is also reflected in their per capita health expenditure, which was USD 28 in Ethiopia and USD 48 in Rwanda in 2016. Especially in Rwanda, out-of-pocket expenditure (OOPE) has reduced although $50 \%$ of the current health expenditure is financed through development assistance. (World Health Organization 2019b) Both countries have invested in the 
HWF. Both have about 8.4 nurses and midwifery personnel per 10,000 population, and about 1 doctor per 10,000 population (Rwanda slightly more at 1.3). While the formal workforce employment has increased over time, there have been likewise huge investments in cHWs, with both domestic and external finance for this workforce available. (World Health Organization 2019d) The crux of the matter is assessing whether such a workforce expansion is sustainable and if it leads to a skilled, accessible HWF in the long term. Ethiopia has seen a GDP growth of about $8 \%$ per year over the last decade and for Rwanda, this economic growth trend has been somewhat similar. A major question is whether such economic growth in LMICs will develop into a domestic scientific, economic, and industrial capacity to generate 'indigenous' labor capacity and production to sustain welfare and wellbeing, including in the health sector. Rodrik's argument is that "Many (if not most) developing nations are becoming service economies without having had a proper experience of industrialization, a process known as premature deindustrialization”. (Rodrik 2017, pp. 9092) This would imply for countries such as Rwanda and Ethiopia that they can't rely on traditional industrial growth paths as those seen historically in Europe, America, and East Asia. This also explains why there is so much focus on the health sector (a service economy) as a driver for employment and growth in LMICs. Rodrik promotes such an approach via massive economy-wide investments in human capital (such as the HWF) and institutions. This has to be a comprehensive reform strategy to invest in the soft service infrastructure via learning and institutional capabilities rather than physical infrastructure (machinery, roads, hospitals, etc.). (Rodrik 2017, pp. 92-93) Rodrik looks specifically at Ethiopia, Rwanda, Uganda and notes that despite improved governance and institutions, and despite investment in health sector employment and human capital, the transformation has been limited. It is projected that in the next decade only one in four African youth will find regular employment as a salaried worker. These youth expect to find good jobs without their countries greatly expanding the capacity to deliver these jobs. The focus in many LMICs should not be on rapid growth figures through commodity booms and external financial investments but rather on structural transformation and social inclusion. (Rodrik 2017, pp. 242-47) The health sector has an important role to play in facilitating such inclusive and democratic processes. Ethiopia has reformed its Primary Health Care (PHC) system and HWF. Several scholars acknowledge that it is not only economic growth that is required to sustain these gains, but that it is also socio-economic development, peace, and stability. There has been political turmoil since 2015 and this challenges the retention of health workers in rural areas. Democratization, social justice, and economic equity are important drivers to systems sustainability (Assefa et al. 2018), more even perhaps than economic growth. For Rwanda, a slightly different analysis can be made. Dussault questions the Rwandan focus on specialist training, academic twinning approaches for capacity development, and vertical program initiatives over PHC development, HWF retention, and long-term health 
systems policy transformation and regulation. (Dussault 2019) While there has been a considerable amount of capacity building in Rwanda, this seems mainly at the medical and individual care level. Rwanda, through aid initiatives, has developed a large community HWF. There are questions on sustainability now that the United States withdrew a considerable amount of their support to PEPFAR (The United States President's Emergency Plan for AIDS Relief). Both Rwanda and Ethiopia position their health systems to be example health models for other African countries. The Guinean government has also been inspired by the Ethiopian Health Extension Program with the aim to strengthen PHC through extensive community outreach. (Kolie et al. 2019) On reviewing available literature, it appears that Ethiopia's system is more solid and that reform has been more transformative compared with Rwanda's. Guinea also has seen considerable economic growth over the last few years, with a projected $5.9 \%$ GDP increase in 2019. (International Monetary Fund 2019a) There is rapid growth but whether there will be structural transformation and social inclusion, also in relation to the health system, is uncertain. Transformation in relation to HWF governance would relate to a decentralized autonomy for health authorities to contract health workers in rural areas as well as an inter-departmental, inter-sectoral committee to plan, implement, and monitor HRH deployment and reform. This process seems to be in a gridlock after an initial momentum post-Ebola in 2016. (Kolie et al. 2019)

Premature de-industrialization, a side effect of hyper-globalization, seems to be mostly taking place in Sub-Saharan Africa as well as Latin America. (Rodrik 2017, pp. 90-91) It is interesting to see what has happened to the health sector in some of the Latin American countries that were covered in the HRH tracing study in chapter 3. While GDP growth rates were up to $5-10 \%$ about a decade ago, this has faltered considerably in several countries. This drop is related to a drop in global commodity prices. (International Monetary Fund 2019a) Latin-American countries included in the HRH study were Ecuador, Brazil, and Argentina amongst others. They have all invested considerably in the HWF over the last decade, including in retention, management, education, skills-mix optimization, and including buy-in and political engagement from the governments in charge at that moment. (Remco van de Pas et al. 2017b supplementary online data) With the economy falling and given widespread protests, it would be relevant to see whether countercyclical economic investments and transformation in the health systems are possible. This depends partly on fiscal space and conditionality agreed upon with International Financial Institutions, something that I'll return to in the discussion part on global governance and the role of external policy actors. In the case of Brazil and the lackluster health sector response to the Zika epidemic in 2016, Gomez and colleagues argue that the focus of the government had been to address determinants of health to alleviate poverty and hunger. In contrast, funding basic health care infrastructure and human resources was increasingly assigned to states and municipalities who had limited fiscal capacity 
to actually do so. (Gómez et al. 2018) Despite investments in the Mais Médicos (more doctors) program in 2010-2015, this policy focus was abandoned due to corruption and political scandals. Since a new government is in power since 2016, there has been an adoption of neoliberal policies which entailed decreasing spending for the MoH. (Gómez et al. 2018)

Researchers from Argentina's MoH came to the observation that while the country has nominal UHC it does not have effective UHC. They have identified 4 key issues for reform: 1. Establishment of provincial public insurance schemes; 2. Creation of a public deliberative process; 3 . Reducing disparities in effective coverage; and 4 . The building of a PHC-oriented system. Especially for the latter, investment is needed to establish 'core family-health teams' (a general doctor, a nurse, and two to three CHWs for approximately 1,000 households or 3,500 people). (Rubinstein et al. 2018) Unfortunately, this is very unlikely as Argentina is in deep economic trouble as it has a USD 88 billion debt to be repaid to international creditors and the International Monetary Fund (IMF). (Elliott 2019) Economists argue that Argentina should embark on "a third path, by developing a homegrown adjustment and reform program that places greater emphasis on protecting the most vulnerable segments of society... Given the downturn in the global economy and the rising risk of global financial volatility, there is no time to waste. Managing a domestic-led recovery will not be easy, but it is achievable-and far better than the alternatives." (El-Erian 2019) Lastly, in Ecuador, the government embarked on an ambitious, broader HRH reform plan about 10 years ago. This also figured in the HRH tracing study and consisted of specialization possibilities, job security program and the inclusion of ethnic minorities within the workforce. (Dal Poz et al. 2015) Nevertheless, the commodity-driven economic boom has ended in Ecuador as well. The year 2019 saw much unrest in the country due to popular protests against austerity measures. The health sector, of which health worker salaries account for $60 \%$ of its expenditure, is bureaucratic and could improve much in efficiency. Specifically, it should move towards a unified health financing system. Something that has not been achieved during the last decade. There is a real risk now that upcoming austerity measures will also affect job security and salaries in the HWF. (Lucio et al. 2019)

In sum, despite the fact that several African and Latin-American countries have attempted to invest, govern, and develop their HWF in the last decade, it has proven difficult to structurally reform the health sector. Besides a range of different factors at the domestic level, the phenomenon of premature deindustrialization might provide an explanation for this. (Rodrik 2017, pp. 246-47) This 'structural' macroeconomic issue is beyond most domestic global health and HRH governance influences but ultimately has a great impact. It is hence not strange that the reverse argument is made by the WHO and others that public investment in the HWF is beneficial for 
inclusive economic growth. (Remco van de Pas et al. 2018) Whilst there is a range of (historical) evidence for this in Europe and East-Asia (Stuckler and Basu 2013), there is much less indication for this in Latin-America and Africa. Rodrik provides nevertheless an argument for going 'back to fundamentals' in LMICs by focusing on public investment in skills-intensive services, the health care sector being one of these. Secondly, he argues for much more public-funded innovation in new technologies so that the benefits of these serve the common good rather than private wealth. (Rodrik 2017, pp. 246-63) In a similar sense, the development economist Rick Rowden argues for more public investment banks in LMICs and heterodox economic approaches (e.g., those that accept relative higher public debt deficits and inflation rates) than the major, orthodox, macro-economic stability policies preached by the IMF and others. Such a heterodox public-investment approach could prevent wagebill restrictions for the HWF and lead to more bottom-up innovation and domestic economic growth. (Rowden 2019)

\subsection{DEMOGRATIC LEGITIMACY}

In chapter 4, I have analyzed the reform process of the wHO from a democratic legitimacy lens and separately analyzed the relevance and effectiveness of the implementation of its Global Code of Practice (on the international recruitment of health personnel). I have conducted these studies early on in the thesis process. Since then, new policy developments have taken place that deserves reflection. Global HWF governance is the second political space in Kickbusch' governance framework while in Rodrik's political trilemma, the functioning of the wHO, and more specifically how it addresses HWF migration, could be seen as part of the embedded liberalism and complex multilateralism approach so much in vogue since the '90s. Nevertheless, I will argue that there are considerable challenges for the WHO to continue its normative function properly.

\subsubsection{The democratic legitimacy of the World Health Organization}

In chapter 4, I have analyzed pathways for the wHO to improve its democratic legitimacy and address its financial difficulties. I have proposed that the wHO should put more focus on democratic principles and less on upholding national sovereignty. More specifically, it would be needed that the wHO improves its input legitimacy (besides output functions such as accountability, transparency, and effectiveness) to increase public trust in the organization. This would require improved deliberation both at the member state as well as international levels to decide what would be priorities for the organization. Although all Member States are represented in the governance of the wHO via the World Health Assembly, it is in reality through 
earmarked funding by (some) Member States and others that priorities are defined. (Legge et al. 2017) Unfortunately, that financial situation, a form of multilateral gridlock hasn't improved much over the subsequent 5 years. Despite several reform proposals, as well as technical improvements in the financing and accounting mechanisms of the organization, is the wHO hampered by "the political-economy of state sovereignty". (Reddy et al. 2018) Firstly, there is still a zero nominal growth policy on assessed (member state) funding. This is blocked partly because some states might have certain economic and business interests that may be hampered by enhanced normative guidance by the wHO (e.g. in the regulation of food products). Secondly, there is currently a politics of withdrawal from international cooperation. One sees a reluctance of states to trust and hence invest in global multilateral institutions. Reddy et al. make the case that "states are reticent to invest in the core budget of the WHO as by doing so they are partly relinquishing their state sovereignty". (Reddy et al. 2018) This conundrum exists until now. Despite the wHo having made an elaborated 'investment case and impact framework' aiming to nudge Member States to invest in the organization as well as having developed innovative finance mechanisms to attract non-state funding, it still struggles to get its budget financed. The wHO's value to Member States remains in question and hence it keeps depending on voluntary, earmarked contributions. (Horton 2019)

This seems to indicate that (contrary to what I wrote in 2014), democratic space, within a complex multilateral arena such as global health and with the wHO as a core institution, is decreasing rather than expanding. Ideally, the wHo could be the 'meta-governor' in networked GHG but that principle is more honored in the breach than in the observance. (van Belle et al. 2018) After several years of negotiations, The World Health Assembly adopted a 'Framework of engagement with non-State actors' (FENSA) in 2016. This framework manages the risks for the wHO engaging formally with a broad range of different non-state actors, including NGOs, the private sector, philanthropy, and academia. While FENSA technically 'democratizes' policy deliberation at the WHO, it does create a kind of 'level-playing field' in which representation may more be dependent on the financial weight that the actors would be willing to put on the table. Buse and Hawkes argue that it will entail "a shift from treating FENSA as a technocratic and managerial project to the political one that it patently is". (Buse and Hawkes 2016) This is needed to protect from and govern the health impact of Big Industry. They argue for the wHO to take sides and actively collaborate with governments and public interest NGOs. (Buse and Hawkes 2016) From this viewpoint, it is interesting to see how the wHO has developed its collaboration with actors working on HWF migration. 


\subsubsection{The governance of international health workforce migration}

In the study in chapter 4, I have analyzed that there are differences between the implementation of the Global Code in Europe, vis-à-vis the implementation in the Eastern and Southern African (ESA) region. This difference can be explained by the fact that within Europe, a civil society and a European Union (EU) 'Joint Action' program was funded to address the transnational effects of HWF mobility. In the ESA region, on the other hand, there was relatively little action by the government and others working with the Code. There is a perception that African interests are not taken seriously by the global health community, including funding agencies from Europe and the United States. Promises and pledges for health systems strengthening have not been fulfilled. Together with colleagues, I posited that perhaps there may be simply more urgent issues to be addressed than health care workers' migration, and in the short run, it might even be beneficial to have migrated health workers send their remittances home. (Van de Pas et al. 2016) Regardless, the most impeding factor seems to be that there is neither a financial incentive nor any form of sanctions that can leverage adherence to the Code. Despite it being a relevant international legal instrument, the voluntary non-binding nature of the Code may not incite countries to take action or report on it. (Taylor and Dhillon 2011) Moreover, original demands by governments from source countries for redistributional compensation measures by receiving countries have not been met. There is a lack of prominence of the Global Code in source counties. Moreover, the 'push' factor of inadequately financed or administered health systems is not addressed in the Code as such. Bourgeault argues, like I do, that "the Code risks having little impact on its laudable goal of ensuring ethical and equitable health worker migration". (Bourgeault et al. 2016) Aluttis also concludes that incentives to change current policies under the Global Code are small and that global power relations are skewed as such that too many high-income countries (HICs) benefit from unregulated HWF migration. (Aluttis et al. 2014)

The study in chapter 4 was used as evidence in the first formal review process of the Global Code of Practice in 2015. (World Health Organization 2015a) A second review process of the Global Code takes takes place in 2020. Partly related to its strategy to work in closer collaboration with the Organisation for Economic Cooperation and Development (OECD) and the International Labour Organisation (ILO), the wHO has also established an International Platform on Health Workforce Mobility (IPHWM). I took part in its first meeting in 2018. (World Health Organization 2018c) The IPHWM was established so as to deepen dialogue on HWF mobility, maximize its benefits, and strengthen the implementation of the Global Code as well as to make the link to the United Nation's (UN's) Global Compact for Safe, Orderly, and Regular Migration. (Campbell 2018) Interestingly, this platform can be considered a type of governance 
innovation as it enabled a broadening of actors involved in the policy dialogue concerning HWF migration. Given the increase in migration of doctors and nurses to OECD countries by $60 \%$ over the last 10 years as well as a complex blurring pattern of mobility between source and destination countries (Campbell 2018), it is greatly necessary that the dialogue between sectors and actors broadens. This adaptive governance mechanism (IPHWM) consisting of both Member States, as well as a broad range of non-state actors, is now tasked to take the issue further beyond the existing 'gridlock' whereby there is limited finance and political willingness by state governments to make HWF migration a policy priority. One of the difficulties of the IPHMW is that beyond the deliberative and dialogue space, there is no agreed approach or normative perspective on how to address HWF migration and its governance. While Member States remain formally in the driving seat, policy entrepreneurs are able to shape the agenda to their interest by means of financial capacity. As an example, the Educational Commission for Foreign Medical Graduates, a United States (US)-based for-profit organization facilitating certification for migrant doctors is active within the IPHWM, and together with the WHO, has co-organized the first global symposium on HWF regulation and accreditation in December 2019. (World Health Organization 2019c) Arguably, this approach facilitates and deepens further migration and international workforce mobility by using an investment- and human-capitalperspective but somehow neglects the equity dimension and potential downsides.

Yeates and Pillinger acknowledge that there is more multi-stakeholder participation in the implementation and monitoring of the Global Code. There are also successes beyond Europe as in the Andean and Association of Southeast Asian Nations (ASEAN) networks where the Code led to a framework for a dialogue on health worker mobility. (Yeates and Pillinger 2019) Nevertheless, in addition to the structural challenges mentioned above, they point to the limited financial and human capacity of the wHO and many governments. Rather than focusing on the adherence of the Global Code, one must consider the root causes of the HWF shortage. There has been much pressure on national health systems in the aftermath of the global economic crisis (2008/2009) that disproportionally affected LMICs. This pressure led to "push" factors for health workers looking for occupations outside their country of origin. (Yeates and Pillinger 2019, pp. 123-25) Also, these authors refer to the expanding growth of the private labor recruitment and staffing industry. The reasons are clear. This international market is highly profitable. Until now there are, despite several codes by the sector itself, limited to none statutory obligations or enforcement mechanisms that force recruitment agencies to change unethical practices. It is suggested by Yeates and Pillinger that such practices could be regulated under a 'UN Treaty on Transnational Corporations and Other Business Enterprises'. (Yeates and Pillinger 2019, pp. 129-33) Such a treaty is under negotiation since 2017 but several European countries, the US, and Canada have so far rejected these proposals and frustrated the process. (Wetzels 2019) 
Returning to our original framework and as outlined above, the two studies in chapter 4 have indicated that there has been institutional reform and innovation in the overall governance and functioning of the WHO, including in the way it addresses HWF migration and recruitment through its Global Code. Nevertheless, this governance space of complex multilateralism is strongly influenced by economic interests by sovereign nation states and other private actors. I analyze that there is much focus on the effectiveness, transparency, and accountability (output legitimacy) but that actual democratic representation and deliberation (input legitimacy) on normative directions and policy choices remain shallow and that such mechanisms (such as FENSA and IPHWM) even obfuscate the economic powers that hinder more robust regulation of commercial interests. Rodrik's political trilemma indicates that in global HWF governance also, the pressures from economic hyper-globalization as well as adherence to nation state sovereignty (by most of the WHO Member States) dominate policy-making and its implementation over democratic principles.

\subsection{INTERNATIONAL POLICY TRENDS OUTSIDE THE GLOBAL HEALTH DOMAIN}

Global HWF development and its governance is a complex inter-sectoral policy field. Notably, Financial-, Labor-, Security-, Development-, Trade-, and Educationalpolicies need to be coherent with broader Public Health objectives. The LancetUniversity of Oslo (UiO) Commission on Global Governance for Health analyzed the impact of other domains on global health policy (without specifying this for the workforce). This commission considered health as a political challenge, not merely a technical outcome. "Global governance for health is achieved when we obtain a fair and equitable global governance system, based on a more democratic distribution of political and economic power that is socially and environmentally sustainable." (Ottersen et al. 2014) The commission looked into seven policy-intervention areas in which the existing system of global governance failed to promote or protect health. Two of these policy-intervention areas seem to be most relevant for the HWF while a third one may become more important in the future. The first of these two policy areas is the financial crisis (from 2008/2009) and its related austerity measures, while the second one is armed violence and impact on health. The third area is related to the impact of investment treaties on health services. In Chapter 5 I have mainly looked into the security (armed violence) and partly the economics (austerity) elements impacting the HWF but in chapter 6, a specific analysis has been included on the link between HWF development and economic growth that I will subsequently address in this section. For reasons of structure, I will look into the security and economics as separate global policy issues, albeit there is overlap in a real-life situation. At the end of this section, I will briefly discuss the relationship between trade policy and HWF 
development as this may become an emerging challenge. I will position the security, economics, and trade elements in the GHG and political trilemma framework that is used as an analytical lens throughout the thesis.

\subsubsection{The securitization of health and its impact on the health workforce}

The main study in chapter 5 looked into the question of whether the securitization of health phenomena could be an explanation for the, seemingly, increasing trend of attacks against humanitarian care workers. In addition, it assessed whether there had been an erosion of humanitarian space for aid workers to provide care. The analysis of the trends, as well as the empirical case studies, does confirm that there is some claim for both hypotheses. Caution must be made here as there are very different contextual and multiple drivers influencing this violence. Part of the increased violence can be explained by a 'blurring' between state, civil (humanitarian) and military actors working in health emergencies and situations of conflict. (KamradtScott et al. 2016) 'Modern' global health issues (e.g. antimicrobial resistance; AMR), Ebola, Endocrine Disruptive Chemicals) are considered global risks in times of globalization. Likewise, these issues have become securitized in the sense that their emergency and 'crisis' characteristics legitimize related health responses bypassing democratic procedures and scrutiny. (Roemer-Mahler and Elbe 2016) Such a health crisis might also legitimize humanitarian intervention in foreign countries. Nunes, while reflecting on the Ebola epidemic, made it clear that global health risks are not distributed in an even matter and this follows patterns of injustice and power imbalances. (Nunes 2016) Against this background, and taking into consideration that several humanitarian actions over the last decade were also part of western, mainly American, but not exclusive, 'smart power' strategies (McInnes and Rushton 2014), it is understandable that this might lead to a form of mistrust against aid workers. To an extent this has always been the case as humanitarian workers, as did missionary health workers in the past, bring with them a set of values and, unwittingly, also a form of "biopower". Humanitarian action can hence be considered part of the "numerous and diverse techniques for achieving the subjugations of bodies and the control of populations". (Foucault et al. 2008)

The ongoing 2019 Ebola epidemic in the eastern part of the Democratic Republic of the Congo (DRC), unfortunately, confirms this pattern. In 2019, the wHO has documented more than 300 attacks on health care facilities that have caused 6 deaths and 70 injuries of health care workers and patients in the DRC. Globally, the WHO has recorded so-far in 2019, almost 1,000 attacks against health care workers while 178 health staff died. (World Health Organization 2019e) While it seems that humanitarian space and related protection of health care workers under humanitarian law is shrinking, one must take also a broader perspective. Over the 
last decade has violence, forced migration and conflict increased in the world. In 2019, 132 million people required humanitarian assistance in 42 countries. Nearly USD 22 billion is required, while the financial shortfall is expected to be about $40 \%$. (UNOCHA 2019) According to the Crisis Group, there hasn't only been an increase in war and political influence. It also states that many actors, both government and non-government, are deepening human misery and deliberately inflicting pain on civilians and use political or military tactics despite the enormous human cost. (Crisis Group 2019) The perspective on attacks against health care workers in conflict (AHCC) has to be seen in relation to this broader trend of humanitarian fallout and militarization. Zimmerman and colleagues provide an insightful reflection that, besides underreporting of the phenomena, there is a particular simplified reporting on AHCC as produced by a small group of powerful actors. This 'framing' of the issue by a powerful epistemic community neglects the complexity of this trend and local drivers for conflict. (Zimmerman et al. 2019) Political agendas drive the reporting as well as the silencing of AHCG. For instance, there has been a relative neglect of Yemen's AHCC vis-à-vis those in Syria. The authors argue that this has to do with geopolitical interests and funding received by the Saudi-led alliance bombing Yemen militias via Western countries. Moreover, non-governmental organizations (NGOs) like Médecins Sans Frontières (MSF), International Committee of the Red Cross (ICRC), and Physicians for Human Rights have their advocacy agenda as well by reporting on AHCC. Potentially, this could backfire as these reports are referred to in resolutions by the United Nations Security Council (UNSC) and are considered illegitimate and incorrect by part of the security council. The authors argue that there are even risks involved with the increased global attention for AHCC. "It is possible that exceptionalist treatment of particular forms of AHCC may create a perverse incentive structure that encourages armed actors seeking global attention to engage in these acts." (Zimmerman et al. 2019) This latest finding is coherent with the conclusions from our study that looked into the inter-relation of the securitization of health, humanitarian space and AHCC. Indeed, humanitarian space is a complex political, military, and legal arena. The ultimate responsibility for ensuring respect for humanitarian principles, including prevention of AHCC, does not lie with humanitarian and health organizations but rather with political authorities and military forces. (Collinson and Elhawary 2012)

The Lancet-UiO Commission clarifies that the global governance mechanisms such as the Geneva Conventions were designed to address conflict between nation states and are less suited to violence committed by non-state actors against citizens and health care workers. Moreover, the different global governance mechanisms are compartmentalized into issues of security, justice, and economic stresses, rather than being developed through a cross-sectoral, integrated approach. The commission argues that issues of global (economic) inequities and injustice must be addressed, not merely overt conflicts and violence, as all of them are key determinants of stable 
and healthy societies. Nevertheless, democratic deficiencies (such as the lack of non-state actor representation or deliberation in the UN Security Council) or weak accountability and enforcement mechanisms in relation to resolutions and security treaties, makes is currently very difficult to govern the conflict and health care domain. (Ottersen et al. 2014) It is likely that most of the reported AHCC will not face juridical prosecution by national or international courts.

\subsubsection{The legitimacy of the health care workers}

In the study on AHCC, my main argument is that the securitization of health and the blurring of spaces between humanitarian and even military objectives potentially impact on the trust in, and the professional legitimacy of, health care professionals working in conflict settings. What deserves further empirical study is a phenomenon explained by Elbe and concerns the 'medicalization of insecurity'. He argues that the rise of health security is also changing who practices security. This leads to medical and health professionals to be more closely involved in the analysis and formulation of security policies. As an example, he mentions the position of international health advisors in national security councils and the rise of health security programs in long-established foreign policy and security think tanks which are run by medical and health experts. (Elbe 2012) Indeed, the international policy debate on the necessity and urgency of certain health security interventions, e.g., in relation to immunization programs or rapid response interventions in disease outbreaks, also demonstrate democratic deficits. While this trend is part of a general democratic recession seen in countries across the globe (Diamond 2015) it might possibly have a spillover effect on the HWF as well. The medical profession is in many countries protected and regulated via a professional council including its own ethical codes of practice. Nevertheless, history tells us that the medical profession has contributed to (while also many opposed and resisted) terror and oppression. (Kolb et al. 2012) It is not immune to political and societal trends. The securitization of health deserves scrutiny within the medical profession as well as how it is perceived within society.

This scrutiny and need for deliberation concerning health emergencies is also the argument that I put forward in the accompanying two analyses in chapter 5. In the analysis of interrogating resilience in health systems development, I argue that the resilience discourse, tying together the security and emergency concept, maintains a status quo and must be understood in the context of rolling out neoliberal governmentality. The focus on resilience is hence not the answer to health systems strengthening and HWF development. I would further argue that it is even antagonistic to the cosmopolitan outlook by its focus on health systems adaptation and responsiveness based on sovereign, national judiciaries. There is as such no space in the resilient health systems perspective for shared responsibility measures or a 
notion of global public goods. Abimbola and Topp argue in an overview article that health system strengthening is a precondition for health system resilience furthered this discussion. They call this 'adaptation with robustness'. (Abimbola and Topp 2018) In the analysis of the Ebola epidemic, I also argue that national health systems should be both adaptive and strong. However, and often neglected in many analyses in this domain, I argue for a post-Westphalian shared responsibility approach based on cosmopolitan ethics via deepening international cooperation and financing mechanisms. Now looking back, 5 years after the Ebola outbreak in West-Africa, this shared responsibility approach seems a distant ideal. The cosmopolitan moment and 'political revolution in global health' (Kickbusch and Reddy 2015) never really took off. There is however an active debate on this, including whether investments in global health security enable health systems strengthening. I will return to this debate when I discuss if and how global HWF governance is able to move beyond the 'gridlock'. Returning to our governance and trilemma framework, I would argue that the securitization (of health) and globalization drivers, rooted in national sovereign governance models (like the wHO's International Health Regulations; IHR) provide such a pressure that the democratic, deliberative space for developing the workforce, both at national and international levels, is undermined.

\subsubsection{Structural adjustment policies}

Both the article in chapter 5 on preventing the Ebola outbreak through HSS, as well as the article in chapter 6 on moving the HWF agenda beyond economic growth, allude to the fact that conditionalities imposed by International Financial Institutions (IFIs) still have a considerable impact on fiscal space available to finance HWF development. The impact of Structural Adjustment Programs (SAPs) imposed by the IMF and the World Bank (WB), including their impact (austerity) on the health sector in LMICs, has been discussed at length in the literature. The debate was originally concerning wage bill ceilings and how this impedes health systems' goals. (McCoy et al. 2008a) Stubbs et al. conducted a cross-national analysis on the IMF conditionality in 16 West-African countries (1995-2014) and arrived at the conclusion that this conditionality reduced considerable government health expenditure in these countries. Basically, there are three pathways identified as to how economic conditionality impacts health: 1. Macroeconomic targets to reduce fiscal space; 2. Wage bills and employment ceilings; and 3. Decentralization measures that amplify budget execution challenges. For the HWF the second pathway matters the most directly, although the others have an indirect effect as well. Kentikelenis provides a framework on how structural adjustment impacts on health systems, deepening and confirming these pathways. (Kentikelenis 2017) 
Over the years, the IMF has acknowledged that it has caused damage to public systems and economic recovery by imposing too stringent conditionalities. For instance, in the case of Greece, it did acknowledge a mea culpa as its austerity measures caused a deep recession in the wake of the 2010 financial crisis. (Brunswijck 2018) In 2019, the IMF released a report that clarifies how it should engage with, and scale-up, social spending in its lending programs. By now, it uses social protection 'floors' in IMFsupported programs and will focus on sustainable and inclusive growth as drivers attaining the Sustainable Development Goals (SDGs), specifically in the field of education and health. (International Monetary Fund 2019b) In a similar trend has the WB reframed its approach and now advocates for investments in Human Capital, including creating new social contracts and financing social inclusion programs. The WB support investing in skills creation to enhance and innovate in the health labor market. (World Bank Group 2019) This aligns closely with the wHO's five-year action plan for health employment and inclusive economic growth (2017-2021). In this action plan, it argues for "raising adequate funding from domestic and international sources... as to invest in the right skills, decent working conditions and an appropriate number of health workers." (World Health Organization 2018a) Seemingly, Major IFIs like the WB and the IMF realize that they require returning to a more pro-public Neo-Keynesian economic investment model. The devil is however in the details. Critical scholars are skeptic about the direction. Ortiz, former director of ILO's social protection program writes "This minimal view of social policy reflects the Washington Consensus, it contradicts international conventions, standards and agreements, including human rights and the SDGs". (Ortiz 2019) Likewise, there is criticism on the human capital approach. The Human Capital Index (an index proposed by the wB) subsumes healthcare to economic concerns. Stein raises concerns on equity and responsibility, as investments in human capital become individualized. Moreover, it lends itself to the development of new debt instruments, as part of the wider financialization of health trends. One sees these instruments already in the financing of university and medical education. (Stein and Sridhar 2019) Many health care professionals in the US, United Kingdom (UK) and the Netherlands (NL) graduate with a considerable financial debt that they require to pay off during their career. These mechanisms have been proposed in the Sub-Saharan context as well. (Reddi et al. 2012) The human capital approach opens the doors to further indebting healthcare practitioners and patients. (Stein and Sridhar 2019)

I share a similar concern on indications that, in general, the fiscal space (for financing decent employment in the health care sector) will not soon be more flexible in the current economic policy environment. Ortiz and Cummins write that austerity policies have become the 'new normal'. In an update and expansion of a global crosscountry analysis on public expenditure and austerity trends, they predict that in 2021 , 130 countries are impacted by an adjustment shock and that the developing world 
will be most severely affected. For the health care sector, this will imply that in 2021 there will be adjustment considerations in "cutting or capping the public sector wage bill, including the number and salaries of teachers, health workers and civil servants delivering public services(in 80 countries)" as well as"...healthcare reforms (in 33 countries).” These public adjustments policies are used as a Trojan horse to induce Washington Consensus policies to cut back on public policies and the welfare state. (Ortiz and Cummins 2019) Indeed, more specific reports such as by Eurodad found that conditionality and advice on wage bills (for the health care sector) are still widespread in the IMF loan programs. Twenty-three out of Twenty-six LMICs' IMF programs sampled by Eurodad are geared towards fiscal consolidation, not towards development and human rights objectives, including the right to health. It is in stark contrast to the IMF claims that conditions have been 'streamlined' and adjusted to promoted social spending. Eurodad recommends (1) creating fiscal space for the health care sector through debt restructuring and (2) that the IMF should respect democratic ownership and stop applying conditions other than the repayment of these loans. (Brunswijck 2018) Researchers from the Wemos foundation came to a somewhat similar conclusion when they analyzed fiscal space for 3 LMICs (Malawi, Uganda, and Tanzania) where the NGO cooperates on health systems strengthening. In all the three countries there was volatile economic growth (on average $2.3 \%$ for 2010-2016) and far below the target of $7 \%$ set for the least Developed Countries in SDG 8.1. Moreover, fiscal consolidation (as a way to deal with structural debts) was still a prominent policy choice in all countries. In all countries, there was a wage bill freeze or cap. (Meurs et al. 2019) In the analyses in chapters 5 and 6 , I share the conclusions from the authors that a GDP increase does not automatically lead to an increase in health spending. IMF's policy advice still adheres to austerity and pro-cyclical economic development with potentially harmful effects on a country's population health. (Meurs et al. 2019) In summary, despite decadeslong debates and recommendations on allowing more flexible fiscal space for LMICS is structural adjustment, austerity, and fiscal consolidation still very much a reality and hindrance in expanding public health care systems. Moreover, even HIcs in the European region are not immune to these trends. The undemocratic control by IFIS through loan programs and debt restructuring is still very palpable, despite framing the matter differently, including terms as 'human capital investment', 'harnessing sustainable and inclusive growth', and 'engaging with social spending'. This has a considerable impact on advancing Global HWF development, especially in countries where there are immediate public health needs. Following my framework; the focus on national fiscal consolidation, matched with demands by IFI's (IMF/WB specifically) as well as pressures by foreign international investment on reducing taxes and salary levels, leads to an erosion of deliberative policy space in the public health realm. Despite popular democratic demands and a considerable increase in strikes by health care workers in LMICs (Russo et al. 2019), is a restriction on expanding the HWF wage bill more the exception than the rule. 


\subsubsection{Trade agreements and their impact}

I have not focused specifically on the trade and health systems policy interface in this thesis. I will only touch briefly on the issue discussing potential implications in the nearby future. The potential impact on HWF mobility in a liberalized, open labor market, in which mutual qualifications of medical diplomas are recognized, can already be encountered in the EU. The enlargement of the EU in 2004 and 2007 by 12 new Member States has also reinforced "east-west" migration in the healthcare sector. Estimated annual outflows based on intention-to-leave data from these EU12 countries to the west is estimated to be about 3\% of the domestic workforce, mainly medical doctors, dentists, and nurses (Ognyanova 2014) However, this trend deepened considerably after the financial crisis in 2010. For instance, Romania lost half its doctors between 2009 and 2015. (Hervey 2017) Many of these health workers ended up in the UK, working in its National Health Service (NHS). An upcoming Brexit would potentially see $10 \%$ of its medical doctors returning to the EU if no agreement can be reached on the mutual recognition of professional accreditations and the working visa status of this workforce. (Fahy et al. 2019) The difficulty within the EU is that the open labor market is not (really) matched by cross-subsidiary fiscal transfers or minimum salary wage agreements between its Member States. Health systems and social protection remain a national mandate but it functions within a European integrated and open labor market in which its states are primarily responsible for financing and sustaining their health systems and workforce. Ghodsee analyses "Life improves for the doctors and nurses who find better paid work in the west, but this migration places a greater strain on already weak health-care systems. Those left behind must deal with many more patients for shrinking salaries, precipitating a rise in informal payments". (Ghodsee 2019)

Mode 4 of the World Trade Organisation's (WTO's) Global Agreement on Trade in Services (GATS) defines a policy framework regulating trade-related possibilities for temporary cross border movement of service providers. The perceived insignificance of health care services in GATs mode 4, partly excluded by countries from this trade framework, has led policymakers, academics and health advocates to focus on other aspects of the (multilateral) trade governance. Trade in health services is hence an under-researched and under-estimated policy terrain. Nevertheless, recent, unpublished, research by the wHO has indicated that contrary to perceptions countries have slowly opened up to liberalize their health services via commitments to GATs mode 4. (World Health Organization 2018e) Although the WTO-GATS negotiations have been 'frozen' since the Doha round in 2003 countries have deepened the trade commitment and framework in bilateral and regional trade agreement (such as exist within the EU). With progress slow in the Multilateral GATS negotiations, 23 WTO members started in 2013 negotiations on a pluri-lateral 
Trade in Services Agreement (TiSa) with the aim to advance liberalization of trade in services and secure commitments from participants that go beyond those in GATS. Talks are on hold since November 2016 but TiSa could imply a deepening of services liberalization. There is a concern by the public health community, labor unions, and civil society that the HWF will be mainly seen as a "tradable commodity" in opening up services liberalization. (World Health Organization 2018d) The wHO sees a possibility to align a flexible trade framework with ethical health worker mobility through applying and monitoring the wHO's Global Code of Practice in a transparent manner. I am worried that there is likely a possible dominance by the trade and investment approach over the ethical, sustainable development needs of health systems across countries, mainly in LMIC's. This resonates with the analysis by Missoni on the impact of global trade liberalization on health systems advancing UHC. "Global trade liberalization can have negative effects on health systems' capacity to ensure Universal Health Coverage. This is especially true in the current weakness, not to say absence, of governance mechanisms to ensure adequate health protection and promotion in international negotiations and policymaking fora, which often lie outside the control of agencies primarily responsible for public health." (Missoni 2013) In summary, there is currently limited impact of the multilateral trade regime on HWF migration but this might change in the foreseeable future and demands close attention. Many of the agreements take place at the regional (e.g. EU, but more and more other regions in the world). There are also bilateral trade agreements between countries that may include HWF exchanges. Currently, the Philippines has such an agreement with several countries. Nevertheless, it seems in this arena, that the focus on national policy space as well as excluding health care mobility from multilateral wTO trade negotiations implies that, for now, the trade regime has a lesser influence on HWF development and mobility than international security and financial policy influences. This may, however, change in the nearby future with the (health) services sector taking a larger proportion of the global economy.

\subsection{LIMITED COSMOPOLITAN SPACE FOR GLOBAL HEALTH WORKFORGE DEVELOPMENT}

When I started in 2014 to work on this thesis, I was more positive that global governance mechanisms and international cooperation would benefit health systems, workforce migration, and HWF development in an equitable and just manner. This expectation to develop and expand shared-responsibility mechanisms and international solidarity to attain health and social goals across HICs and LMICs has become bleak. At least, the outlook and space towards cosmopolitan approaches have considerably diminished in current global governance mechanisms such as the SDGs, United Nations Security Council, the wHO's Working for Health program 
as well as the WHO's overall $13^{\text {th }}$ Global Program of Work 2019-2023. (World Health Organization 2018g) I have already touched upon several reasons, amongst others a regression of democratic space in many countries, including within the EU. (Diamond 2015) With increasing global risks in the financial, ecological and security realm do societies and political actors withdraw behind their perceived 'safe' national spaces and retreat from multilateralism and shared sovereignty approaches advancing global public goods (such as for health) or addressing global public bads (e.g. climate change). A Dutch think-tank in international affairs observes that the cooperation and rules-based, Western-dominated, multilateral world order is in decline. In the West, there is a trend of populist sovereignty with popular movements that are opposed to the supra-nationalization of governance, policy, and jurisprudence. In its analysis, the think-tank quotes Antonio Gramsci who wrote in the 20s' of the last century "The ruling class has lost its consensus... In this interregnum there arises a great diversity of morbid symptoms." (Sweijs and Pronk 2019) Indeed we live now in a new interregnum and this international chaotic shift ('multi-order') has a considerable impact on the Global Health Governance (GHG) framework and its trilemma. What can be observed in the general trends of the current GHG policy landscape, and more specifically those relevant for the HWF?

\subsubsection{Global health governance grounded in the Right to Health}

In the main study in chapter 6 , I researched with colleagues as to whether GHG in the SDGS is grounded in the Right to Health. Such a rights-based approach is considered as moving the cosmopolitan ethics forward in foreign policy for health. (Lencucha 2013) For this analysis I used and re-ordered Frenk and Moon's four functions of the Global Health System, extrapolating them to global governance for health functions and whether these would allow achievement of the Right to Health. These four functions include stewardship, production of global public goods, mobilization of global solidarity and the management of externalities. (Frenk and Moon 2013) In summary, regarding stewardship, one can observe a trend that GHG continues to be less hierarchical and has become more 'networked'. This phenomenon can also be witnessed in the "Global Action Plan for Healthy Lives and Well-being for All", also known as the SDG 3 GAP. (Voss et al. 2019) This GAP has been developed by the WHO in close collaboration with 12 other global health-related multilateral institutions and should be seen as the 'masterplan' to implement SDG 3. It is built around the principles of 'engage with countries, align efforts, accelerate progress, account for collective results, assess implementation.' (Voss et al. 2019) Despite these lofty words, there are no new financial commitments by governments to implement the Global Action Plan (GAP) and accelerate sustainable financing for health. Amongst the seven 'accelerators' highlighted in the GAP, there are some critical SDG 3 components overlooked; HRH is one of these. (Voss et al. 2019) SDG 3 could be regarded as the 
operationalization framework in producing global public goods for health. Nevertheless, Seidman analyzes that there are select limitations of SDG 3 including there being no or limited reference to PHC as a basis for a strong health system and that it does not track impacts related to financial risk protection. He rightfully questions the theory of change of SDG 3. (Seidman 2017) The last report of the UN Secretary-General on implementing the SDG mentions that progress in the health domain has stalled as at least half the global population does not have access to essential health services. It also states that in 2018 close to $40 \%$ of all countries had fewer than 10 medical doctors per 10,000 people, and more than 55\% had fewer than 40 nursing and midwifery personnel per 10,000 people. (Guterres 2019) I wrote in the GHG study that one of the four functions of the Global health systems is to mobilize global solidarity. However, the third International Conference on Financing for Development rejected the notion of existing common but differentiated responsibilities (a principle that has been evoked in the global climate governance domain) for financing the Sustainable Development agenda. Rather, there is an uncritical embracing of the private sector as well as a naïve hope for domestic resource availability in LMICs that is called upon to make global partnerships (SDG 17) happen. Others analyze that commitments to increase ODA and to improve its quality have not been implemented. Debt service payments of developing countries are proportionally rising, while the rate of taxation relative to GDP has fallen in most LICS. Progress on SDG 17 is conspicuously lagging behind. (Meurs et al. 2019) Statements on international support and partnerships are more honored in the breach than in the observance. Lastly, I argue that managing externalities, or the so-called 'global bads' impacting health seems the worst function being institutionalized from a right-based perspective. Beyond the IHR, there is currently little global governance space to take cooperative action on issues such as air pollution, commercial determinants of health, or armed violence. Three years after the original analysis for this study, this gridlock in global cooperation, from a rights-based perspective, has continued or even deepened. A follow-up research on the role of 'the International Health Partnership for Universal Health Coverage 2030' (UHC2030) in advancing GHG and the Right to Health states that a pure technical approach to advancing UHC risks mirroring the global and national patterns of exclusion and injustice. With ongoing shortfalls in domestic finance, and retreat from international engagements and financing from wealthy countries, it depends on civil society to express demands and pressure to implement the right to health, including its approach for HWF development. (Hammonds et al. 2019) Given a general shrinking of civil society space and democratic regression, it remains to be seen whether this is possible. Remarkably, the human rights discourse has also left the HWF policy domain. The World Health Report 2006 mentioned the need for "global solidarity and advocating ethical recruitment and migrant workers' rights". (World Health Organization 2006, pp. 159-60) The Kampala declaration and agenda for global action talks about upholding human rights principles and upholding the vision 
of health for all. (World Health Organization 2008) The Global Health Workforce Alliance, in preparation for the second global forum on $\mathrm{HRH}$, made a promotional video with the title 'Imagine - a health worker for everyone, everywhere'. (Global Health Workforce Alliance 2011) Less than a decade later, the frame has considerably changed. In the Dublin Declaration on HRH, human rights are not mentioned. On the contrary, the term 'investments' (in a transformative workforce, in human capital) appears frequently. (World Health Organization 2017a) The realization that there has been a shift in the discourse on HWF policy development led me to write the last analysis in chapter 6 , which argues that there are alternative approaches to address the workforce problematic rather than looking at it only from an economic and labor market angle. This analysis deconstructed the economic approach towards HWF development. It came to the same conclusion as the main study and other analyses in chapter 6; that a human rights approach to health has been largely been left out of the SDGs. A global justice (shared responsibility) approach to health systems development and health employment, within ecological limits, could be imagined but this requires paradigmatic change and a real shift in and beyond our GHG and trilemma framework. I will return to this in the last part of the discussion. Nevertheless, there are scholars who have analyzed different possibilities to overcome global health (workforce) governance challenges that require that I reflect, from a cosmopolitan outlook, on such suggestions. The first approach considers the potential that investing in global health security could also strengthen health systems and human security more broadly. The second approach sees global health as a complex adaptive system, and considers adaptive and resilient governance features as a possibility to further global health objectives.

\subsubsection{Synergies and tensions between UHC and global health security}

Kickbusch and Reddy have proposed that the Ebola outbreak in 2014-2015 could imply a cosmopolitan moment in global health, as its containment is considered critical for national and international security, and domestic and global economic well-being. Following Beck's thinking, they propose that an expectation of global risks 'open up a complex moral and political space of responsibility'. (Kickbusch and Reddy 2015) This would imply that there is political momentum for advancing international investments in health systems and managing externalities given the global risks (pandemics, environmental disasters, etc.) that humanity faces. This argument has been expressed by De Swaan: "The faraway poor are not enough of a nuisance, and not enough of a threat for the rich of this world to goad the wealthy into collective action aimed at improving the lot of the indigent in remote areas. Yet, epidemics that are associated with poverty in other parts of the world may one day provoke concerted action by wealthy countries to eradicate the conditions of poverty that caused the spread of disease on a world scale." (De Swaan 1998) This consideration let Ooms and all to propose 
establishing 'a marriage of convenience' and hence ensure that Global Health Security and UHC objectives plus its finance advance in an integrated and coherent manner. (Ooms et al. 2017) It has led to the establishment of a Lancet commission addressing the fragmentation in Global Health. This commission has the aim to overcome fragmentation and realize the potential for coherence in global health. It will systematically examine intersections between these leading agendas in global health, them being UHC, health security, and health promotion. (Ooms et al. 2018) Wenham and colleagues in a similar fashion have constructed a framework in which they argue that such a marriage of convenience between UHC and Global Health Security (GHS) could become a strategic, effective partnership. The authors argue for a conceptual divergence between individual and global security while making the case that HSS can be the policy mechanism, which brings GHS and UHC together. (Wenham et al. 2019) I analyze that the HWF is at the center of their synergetic framework but at the same time, there is very little consideration of how the workforce should be developed to serve HSS, UHC and GHS objectives. (Wenham et al. 2019) Likewise, both in the Lancet Commission on overcoming the fragmentation in global health as well as in the SDG 3 GAP, there is a conspicuous absence of the mechanisms and governance required to develop the HWF in a way that serves the multiple objectives of GHS, UHC and health promotion in an integrated manner. (Ooms et al. 2018; Voss et al. 2019) Despite arguments that the HWF is indispensable for GHS (The Lancet - Editorial 2016) and the policy recommendation by the wHO that IHR should be embedded in national health systems (Kluge et al. 2018), I have not found compelling evidence that current international investments in GHS programs contribute to HWF development on the long term. Of course, there are exceptions to this as for instance observed in Ethiopia and Rwanda but the overall impact of GHS labeled investments on sustainable HWF employment has been limited. Unfortunately, and despite an era of increased global risks, aggregated Development Assistance for Health (DAH) funding by G20 countries has stalled and has slowly decreased over the last years. Albeit in 2018 30\% of all DAH was labeled as HSS, $51.7 \%$ of these funds focused on building health system capacity for a specific health focus area, such as HIV/AIDS or vaccines. "Concerns remain that without meaningful investments in developing PHC systems and strengthening key health system pillars, health gains are less likely to be sustained." (Dieleman et al. 2019) Our empirical work on HWF development in Guinea post-Ebola confirms the comparative analysis above. Epidemic preparedness and response capacity were highly prioritized in the HSS programmatic cooperation and funding in Guinea. Financial inaccessibility to healthcare remains the bottleneck to expanding health services coverage in Guinea. Structural health systems reforms, including in the HWF and health financing mechanisms, have not happened despite all health investments in the country post-Ebola. (Kolie et al. 2019) I assess that, despite intentions and conceptual possibilities, the international investments in health security programs in overall 
do not have a large impact on long-term HSS and its structures. Short-term funding for a more 'narrow' approach to health security considerations prevails over longterm investments in social and human security, including in the development of HSS. This is partly because of the complexity and inter-sectoral approach required to strengthen the HWF and partly due to the political and fiscal space limitations in countries, as well as internationally, to actually invest in the workforce. Global risk distribution, including in health, replicate global injustices. As long as the direct pandemic risks are contained and prevented from spreading to richer countries, as well as richer parts of societies in less affluent countries, then there is not an immediate need to invest in the HWF, social protection, and health systems in the long term.

\subsubsection{Complex adaptive systems}

Following the theory by Hale et al., Kickbusch proposed that there might be a gridlock in global cooperation for health. (Kickbusch and Reddy 2015) In follow-up research, Held and Kickbusch provide the thesis and evidence that global cooperation for health is able to move beyond gridlock. Remarkably, it states "Global health governance is in many ways proving more innovative and resilient than other sectors in global governance." (Held et al. 2019) This conclusion requires closer investigation. More specifically, I am interested in what the position of the HWF is in this analysis. Held and colleagues come to the assessment that growing multi-polarity and institutional fragmentation allows for learning processes in governance innovation, such as the creation of highlevel commissions, e.g. the UN High-Level Commission on Health Employment and Economic Growth (although not directly mentioned by Held). Poly-centricity might also harness institutional diversity and could make GHG more inclusive, for example, by the creation of hybrid institutions such as the Global Fund and many others. In a separate analysis, we came also to a similar conclusion that poly-centricity and networked governance could be beneficial as long as there is clear stewardship, coordination and is best with the wHO as 'meta-governor'. (van Belle et al. 2018) Lastly, Held assesses that political leadership by both state and non-state actors, as well as individuals has been crucial to elicit innovation and engagement to solve complex global health problems. (Held et al. 2019) My main critique of the study is that the three global health cases on which the analysis rests are all in the Global Health Security domain (HIV/AIDS, Ebola, AMR). They hence form a direct global risk for the richer countries and trigger, potentially, a form of realistic cosmopolitanism. (Beck 2006a) Nevertheless, the study does not address indirect risks linked to the absence of social protection for health (UHC), weak health systems, or workforce limitations. This is acknowledged when it states that "many of our interviewees emphasized the importance of pathways that are only partially captured by gridlock theory... continuing the shift away from vertical, disease-specific governance to an approach focused on UHC and 
global public goods". (Held et al. 2019) Remarkably, there is no mention of the HWF, its limitations and the need to include HWF governance and development in adaptive GHG innovations. This is incomprehensible given the crucial role and pillars that $\mathrm{HRH}$ have in making health systems function. There is a conspicuous absence of the HWF in GHG innovation considering its essential role in moving pathways forward toward health for all.

Nevertheless, HWF policy and its governance are included in complex adaptive systems thinking. Especially at the domestic and local levels, there are studies that capture the complexity in HRH governance and performance, whether in LMIC(Dieleman et al. 2011) or HIC-settings (Greenhalgh and Papoutsi 2018). Nevertheless, in the GHG and HWF nexus, this has not been the case. One exception is a study by Best et al. which is a case study on the WHO's Global Health Workforce Alliance (GHWA), the hybrid global health initiative hosted by the wHO and functioned from 2006-2016. (Best et al. 2018) The study analyzed the functioning of GHWA using a complex adaptive systems lens and applied Shiffman's theory of network emergence on the development and effectiveness of global health networks. (Shiffman et al. 2016) The GHWA study-case arrived at some main recommendations for the next stage of global HRH governance. It focuses on effective management and leadership, an adaptive networked governance structure, the use of a vigorous communication strategy, and a clear focus on goals and management. (Best et al. 2018) To me, it is remarkable that the authors approached GHWA specifically, and networked GHG governance in general, as an autonomous system, somewhat devoid of international politics and its shifting context. There is no reference to the role of international finance undermining global health institutions, a phenomenon known as Trojan multilateralism (Sridhar and Woods 2013), nor is there attention to the relative ignorance of the HWF in other GHG domains such as UHC, Global Health Security, and the SDGs. It is naïve to assume that if networked functions of HRH governance improve, that this community will then automatically emerge as an important global health network. I have clarified in this thesis that the emergence of HWF governance along the three spaces is highly influenced by the constraints of the political trilemma, especially the security and economic conditions imposed by hyperglobalization, its straightjacket, and countries upholding their sovereign strategy. To understand these dynamics better, it requires me to assess underlying politics and power structures in GHG, and then specifically in the HWF domain.

\subsubsection{Exerting power in global health governance}

Shiffman, following Bourdieu, makes the case that global health must be seen as a field. Power in this field is not only enforced by financial capital, but also by social (e.g. education credentials), cultural (e.g. networks) and symbolic (e.g. in how 
legitimacy is constructed) capital. (Shiffman 2015) Shiffman and a range of other scholars confirm that overcoming the input legitimacy deficit in global health, e.g. improving deliberative mechanisms and representation, is of utmost importance. (Shiffman 2015) This is coherent with my analysis of the wHO reform in chapter 3. Likewise, applying Rodrik's political trilemma to Global HWF Governance I argue that democratic politics, basically a function securing input and output legitimacy, is mostly under stress. Shiffman also makes the case that the 'Global Health rationality project' is illusory. Issues of power cannot be excluded from the field and 'scientific methods alone cannot resolve fundamental questions in global health, such as what health equity means and how health resources should be allocated.' (Shiffman 2015) More recently, Moon has explained power in GHG, including reflecting on its complex adaptive governance capabilities. She deconstructs eight different types of power and clarifies them with examples from the health domain. (Moon 2019, pp. 5-6 tab.1) In this typology she nuances that these several forms of power are not mutually exclusive and transferable, and that a wide range of actors (like NGOs) can exert power, even without having means. (Moon 2019) Interestingly, neither Shiffman nor Moon or other GHG scholars give attention to power issues in relation to HWF. Global HWF policy development somehow 'escapes' this scrutiny by scholars and is mostly approached as a rational, scientific project. (Stone 1988)

When I apply this to the Global HWF domain, questions can be raised about the legitimacy of, and use of discursive power in, its governance structures including the new IPHWM. (World Health Organization 2018c) I questioned in the last analysis of chapter 6 why there is so much focus on the economic growth perspective in HWF development. This economics perspective is even more 'justified' by the wHO and others providing scientific evidence on the interrelations between investing in the HWF, decent employment, and inclusive economic growth. I have analyzed, and have provided, alternative (economic) perspectives on why states and other actors should take HWF development seriously, including from a security, human rights and global public goods approach. Nevertheless, some scholars do analyze governance and power relations in HFw issues, especially when it comes to migration. Labonté analyses HWF migration and governance mechanisms and points to the sensitivity of global compensation and restitution, and that this is not addressed in the Global Code of Practice. Labonté looks into the concept of Global Skills Partnerships (GSP), based on employers and governments in destination countries financing the training of occupationally desired potential migrants in source countries, and then managing their effective job placements and integration within destination countries. This would not only fund skilled workers to enter a foreign health labor market but would also finance domestic health workers in countries of origin. This 'human capital' approach to migration would almost certainly "require additional financial transfers between countries, however, to ensure decent employment opportunities 
for such health workers in their home countries." (Labonté and Ruckert 2019, p. 206) This analysis is coherent with our own analysis that GSP are a narrow, technical, bilateral approach to address the complex issues and injustices seen in global HWF migration. There is no evidence that GSP are efficient and sustainable in the long term. There is a considerable risk that existing inequities in health systems between and in countries will be reinforced. (Van de Pas and Mans 2018)

Yeates and Pillinger have written an authoritative overview on the global governance of international HWF migration and recruitment. (Yeates and Pillinger 2019) They come to some compelling conclusions that ought to be shared here. Over the years, the overall governance trend has been a shift in the regulatory approach away from more radical forms of progressive redistribution from destination to source countries and towards global 'ethical recruitment' approaches. With the latter, it is implied that HWF mobility becomes a win-win approach for all actors involved. Yeates and Pillinger are likewise critical about controversial proposals for GSP. Given the track record of bilateral agreements, they see little prospect in GSP providing lasting solutions. They assess that "global governance and policy have been neither sufficiently attuned to the scale and urgency of health workforce and health systems crises nor treated these crises sufficiently well as trans-boundary issues connected global capitalist dynamism." (Yeates and Pillinger 2019, p. 207) The 'thickening' of global governance in this domain has not led to HWF sustainability to be considered a shared global solidarity. The authors ask for critical scrutiny of the collective power and extent of the hegemony of rich destination countries in relation to HWF migration policy formulation and governance mechanisms. They also urge researchers and policymakers to look at the interplay between several global governance regimes to fully understand the evolving contours of this global policy field. (Yeates and Pillinger 2019, pp. 216-17) Yeates and Pillinger provide some recommendations on implementing shared global responsibility in HWF governance. Firstly, they focus on the need for strengthening the implementation of existing mechanisms such as the wHO's Global Code and ILO Conventions on labor migration and recruitment standards. Secondly, new instruments for the renewal of global HWF governance are required. A Global Health Resource Fund (Mackey and Liang 2013) is a mechanism that could deserve attention and potential support. While the authors do not support standalone global health funds, they do argue that mechanisms in which financial restitution transfers to source countries for their lost investments in education and training must be feasible. These mechanisms could also support Hss including increased public expenditure for HWF employment. Thirdly, the authors argue for a socially-progressive trade agenda in which the International Financial Institutions, the WTO and other multilateral institutions adhere to any regulation or agreement to respect fundamental rights at work, ethical recruitment, and fair migration. "Instead of social clauses in international trade agreements, much greater attention needs to 
be given to global social justice and the right to health as core principles of state policy, whether on a national or international scale, accompanied by sustained programmes of resourcing and implementation." (Yeates and Pillinger 2019, p. 220) Lastly, they argue that any labor migration in the care sector, including for instance via a GSP, should be done based on fair treatment of migrant workers and according to ILO's conventions. Reciprocal arrangements should be put in place to ensure that migrant workers have portability of social security and possibilities for re-integration in the care sector. (Yeates and Pillinger 2019) My overall analysis of global HWF governance and requirements, following the cosmopolitan outlook, are mostly coherent with these scholars. The question remains whether there is policy and political space to move forward with such recommendations. In current times of multi-order and nationalistic tendencies, there seems to be little room for cosmopolitan perspectives. There are some glimpses of hope though but these demand paradigmatic change. I will return to this in the last part of the discussion. Before doing so, I need to reflect on the framework constructed as well as the relevance and limitations of the research methodology applied in this thesis.

\subsection{THE POLITICAL TRILEMMA AND GLOBAL HEALTH WORKFORCE GOVERNANCE}

Reflecting on the different elements of the GHG policy space in the previous chapters, and linking them with the Political Trilemma of the world economy, I have arrived at an image that looks as follows (Figure 7.1): 


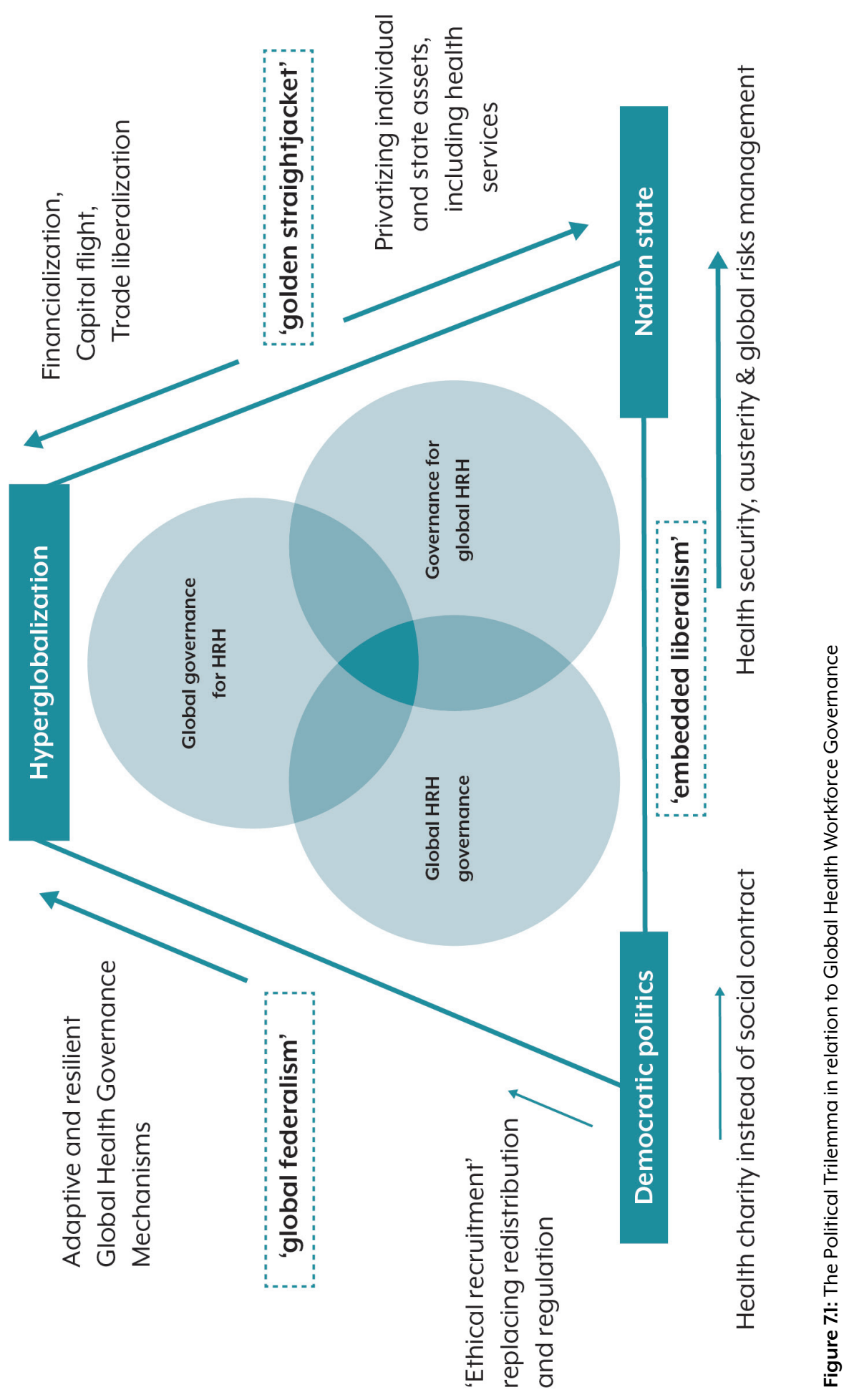


This picture indicates that under the influence of hyperglobalization, the embedded liberalism policies, also known as the 'Bretton Woods compromise', is under considerable pressure. The Bretton-Woods set of global monetary and fiscal regulations, including a role for the ODA, was established with the idea to maintain an open, liberal, and democratic economic order that facilitated a free trade regime. Also, countries would have the freedom to differentiate their policies, including in the domain of taxation and industrial protective as to build their economies and strengthen the welfare state. This has been the bases of prosperity, economic growth, and social protection systems in European states and several South-Eastern Asian countries. Nevertheless, the system has never been just as it relied, and still relies, on cheap labor and raw materials from post-colonial states in Latin America and Africa. Moreover and ironically, during the 40 years of neoliberal politics, this regulatory system has been eroded by the powerful countries that designed it. This is the Globalization Paradox. (Rodrik 2011a) Deregulation in the trade, monetary, and economic domains matched by debt-creation and structural adjustments policy under the Washington consensus has eventually led to what is framed as Neoliberalism 3.0. (Labonté and Ruckert 2019, pp. 62-65) This is less about eliminating the state as much as occupying it. Neoliberalism 3.0 has been successful in creating a neo-gilded age of extreme wealth. (Schrecker 2017) Although criticism of neoliberalism has been increasing, and the world economy has become unstable with cracks appearing in power constellation, neoliberal economic policies have proven to be resilient. (Labonté and Ruckert 2019, pp. 62-65) In this era of hyperglobalization, and under the banner of national sovereignty, there is a tendency to maximize assets and profits by the few that control the state. With increased mobility of capital, information technology and a global reach of banks and stock markets, it has become more difficult to tax wealth. (Milanovic 2016a, pp. 54-55) With reducedtax income from wealth and profits generated by transnational corporations, many states, while adhering to fiscal stability principles, opt for austerity measures in the public sector and welfare state. As a result, and to uphold essential public health functions (health security) and health services, more public-private partnerships are introduced, privatization is encouraged and labor markets become more 'flexible'. (Ortiz and Cummins 2019) Austerity as 'the new normal' is the default mode for many states, including within the EU. There may be alternatives for this that will be touched upon in the last part of the discussion.

The impact of this trilemma on global HWF development and its governance is that there are political-economic constraints outside the health sector itself that impede skilled, decent, and well-distributed employment for health professionals. The analyses from chapter 3 indicate that it is difficult to so on a sustainable basis despite an improved understanding of the functioning of labor markets, better data collection on workforce requirements, and a call for investment in the HWF as part 
of the Sustainable Development Agenda. There is no reliable international financial support mechanism to support health systems in LICS. The WHO, as the main UN multilateral organization working on health, still addresses this global HWF challenge from the 'embedded liberalism' thinking, stressing on 'good governance' and a 'democratic, whole-of-governance' approach. It is not surprising, since the Bretton Wood compromise is one of the main foundations on which the UN functions. Despite some countries like Germany and Norway focusing on the prerogative to maintain multilateral principles for global health and global public goods amongst others, by active support of the SDG 3 GAP there is considerable pressure on this side of the political trilemma. Many countries, under pressure from hyper-globalization and the 'golden straightjacket', are withdrawing from the multilateral compromise. They rather focus on domestic sovereign jurisdiction and aim to provide their 'own' citizens with a minimum form of a social contract, including for health care. Under popular pressure and political manipulation, countries as diverse as the US, UK, Brazil, India, and Turkey have withdrawn themselves slowly from multilateral 'shared sovereignty' politics, including in the health domain. It is remarkable to look at the framing of UHC (e.g. 'Modicare', Socialist 'Obamacare') and the sustainability of health systems in these political developments. They all share a focus on 'taking back control'. The narrative by the conservative UK government in addressing growing staff-shortages in its NHS focuses on getting "Brexit done and introduce an NHS fast-track visa for qualified doctors and nurses-because we value their contribution to our fantastic NHS." (Savage 2019) This populist account neglects, probably, real financial capital interests behind an upcoming Brexit and it is likely that the NHS and health employment will come under severe pressure in the coming years. It will become more difficult to recruit staff within and outside the UK. There are many uncertainties in the mutual recognition of professional qualifications. (Fahy et al. 2019)

Like many others, I struggle to understand why so many citizens in HICs and Middleincome countries (MICs) vote against their own interests, including in relation to social protection policies and health care. Schinkel and van Reekum who provide the metaphor of a 'corral' to describe modern bio-politics provide a convincing argument. They state that the shallow prospect of current social democratic compromises, including the promise of inclusive economic growth and basic welfare, is a partnership between 'Whiteness' and 'Capital'. It is a contract between a precarious middle-class, based on identity politics, and aimed at sustaining current power relations under capitalism. (Schinkel and van Reekum 2019, p. 31)

That there is much discomfort with current democratic politics in several countries is visible in the many demonstrations, riots, and violent conflicts taking place. Whether this is the 'Gillets Jaunes' in France, the Extinction Rebellion (XR) movement, anti-government demonstrations in the Middle-East and Latin America, or even 
the Islamist inspired insurgency in Sahel countries. These are several expressions of growing insecurity, precariousness and a reaction to planetary plundering and climate injustice. The HWF is not immune to this trend. In low-income countries, health workers' strikes have become more frequent in recent years. (Russo et al. 2019) Health workers also contribute to anti-government demonstrations such as in Gaza's 'Great March of Return' and become then at risk being targeted by security forces, as was explained in detail in chapter 5. In 2018, 3 health providers were killed and 560 injured during attacks on healthcare workers and facilities in Gaza. (David Mills et al. 2019) Another example indicates that health professionals have formed a 'Doctors for XR' chapter in the UK. (Extinction Rebellion 2019) These are all indications that the multilateral Bretton Woods compromise has become fragile, including for and within the health care sector.

On the cosmopolitan side of the trilemma, policies based on shared sovereignty and responsibility ought to mitigate the negative impact of hyper-globalization in a democratic, regulatory manner. I would assess that this side of the trilemma is most affected. Deep economic integration, including more and more in the health services, demands cheap and mobile labor. The global health economy is one of the fastest-growing investment sectors, and global healthcare expenditures are likely to continue rising. Its spending is projected to increase from USD 7,724 trillion in 2017 to usD 10,059 trillion in 2020. (Kirton and Kickbusch 2019, p. 17) Nevertheless, I assess, like other scholars, that there has been a shift away from global regulatory approaches that secure labor rights and GPGH in the HWF domain. (Yeates and Pillinger 2019) Rather, the trend indicates moving in the direction of multi-stakeholder partnerships as envisaged under SDG 17 and of which the IPHWM is an example, ethical codes to manage labor mobility (such as the global Code of Practice), corporate social responsibility, and adaptive governance mechanisms. (Held et al. 2019) Chapter 6 of this thesis confirms that this governance space has moved away from a rightsbased approach to global health development. Moreover, in chapter 4 that touched upon the legitimacy of the wHO reform, I have made it clear that input legitimacy (representation, deliberation) is missing in current GHG mechanisms, including for the HWF. Unfortunately, this has not improved in the SDG era. Shiffman, when analyzing the legitimacy of global health networks indentifies positive elements, like the role of civil society and the generation of shared expertise, but certainly also sees some negative elements. These include the control of those networks by 'Northern' elites, with limited representation from 'Southern' institutions, not to mention citizens from Southern countries. Additionally, these networks have contributed to the fragmentation of global and national health governance. (Shiffman 2017) He asks the question of whether the deficiencies of multilateral organizations justify the existence of these 'hybrid' public-private governance networks or rather that they exercise power without legitimate authority. My analysis tends to be more 
supporting the second clause. These new 'GHG' arrangements have not been able to mitigate the externalities of hyper-globalization, including widespread austerity, tax evasion, capital flight and the creation of a 'new' debt burden in LICs. During the 40 years of neoliberal policies and hyper-globalization the HWF has been reduced to a 'human resource', health professionals to 'human capital'. The health services domain has become a labor market. Health care provision is no longer considered a common good, but has become more and more commodified as part of the global health service industry. Moreover, economic externalities and global public 'bads' such as the overshoot of planetary boundaries, the climate crisis, and ecological degradation have not been included in the functioning and consideration of GHG mechanisms.

Kickbusch and Rodrik both offer options to advance problem-solving along the three GHG spaces, respectively overcoming the political trilemma. Kickbusch acknowledges the effect of hyper-globalization by stating that now that the global health industry grows to represent $1 / 8^{\text {th }}$ of global economic flows, GHG institutions must "firmly establish processes to link actors within and between sectors and define firewalls and conflict of interest strategies." Controversial actors can only be involved in the public health domain if there is a commonly agreed rule-based-system for including non-state actors in global governance institutions. Without it, it is difficult to subject powerful organizations such as foundations and corporations to critical analysis. (Kickbusch and Szabo 2014) The ongoing contestation over the WHO's FENSA indicates how difficult it is to arrive at this commonly agreed rule-based-system in GHG based on 'traditional' multilateral governance structures like the UN. (Kent Buse and Hawkes 2016) Rodrik provides seven principles for a new and sane globalization. The essence of these principles is a simple idea that "The reach of global markets must be limited by the scope of their (mostly national) governance. Provided the traffic lights are right, the world economy can function quite well with nation states in the driving seats." (Rodrik 2011a, pp. 13340) Rodrik calls this "Capitalism 3.0" Applying these cosmopolitan, but enlightened self-interest, principles would practically mean that there should be a reform of the international trade regime whereby there is agreement on development and social safeguards and that there is an agreement how to regulate global finance as the current system is one of international discord. He also talks about reaping the benefits of global labor flows. Rodrik, and as we similarly suggested in the GSP proposals (Van de Pas and Mans 2018), support temporary work visa programs to support labor mobility. The health sector plays an important role here. Rodrik acknowledges that a fair, enforceable, global governance regime must accompany such temporary work visa programs. It must be economically attractive to return to the country of origin, have portability of social rights and word under decent labor conditions in the health system of a receiving country. His main argument to support such schemes is that the economic benefits outweigh the negative side 
effects. (Rodrik 2011a, pp. 141-52) These principles are also embodied in the UN Global Compact on Migration. The Migration Compact provides a new undertaking by states to uphold the human rights of migrants. However, "the compact needs to be strengthened by an enhanced commitment to eliminating discrimination between citizens and migrants, and between different categories of migrants." (Guild 2019) Our own analysis of the GSP comes to a somewhat similar conclusion. A decent and just governance mechanism might provide mutual benefits, but the details and political economy of these schemes raise questions about equity and sustainability and about the rights of the domestic HWF ('citizens') vis-à-vis foreign health personnel ('migrants'). There is no systematic evidence yet that the temporary work visa programs in the health sector are fair and sustainable. Albeit these principles are included in the UN Global Compact on Migration as well as the wHO Global Code of Practice they are neither enforceable nor is it possible to hold the several parties accountable when they violate these principles. (Van de Pas and Mans 2018)

In conclusion, while there seems convergence how things should be done to improve the global HWF and its governance, taken into consideration (realistic) cosmopolitan ethics, in reality, this is not how it is. Conflicts of interests, entrenched power issues rooted in colonial legacies, and an unstable multi-order make than nation states rather focus on their own health systems, welfare state, and labor markets, even within the EU, rather than that they take a shared responsibility for a greater common good. Global health how it should be versus global health how it is remains a pervasive ambiguity and schism in modern GHG, including in relation to advancing UHC and HWF development. The gridlock in global health cooperation persists. (Van de Pas 2019) I have tried to address this in one of the analyses in chapter 6, whereby I argue for 'a dual track approach'. "We have to continue with our aim to gradually transform capitalism into something better while in parallel we should be well aware that we need to move beyond resilience and capitalism fast." (Van de Pas 2017) In the first element of this dual track, I agree with Kickbusch and especially Rodrik that it is required to regulate 'Capitalism 3.0' as a pathway to attain the health-related SDGs. However, several years further down the line and seeing how global (health and income) inequalities are growing and the climate crisis is unfolding, my analysis, and intuition, directs that the time is ripe for paradigmatic change if we want to further global common goods. Rodrik relies on open economies and trade, as well as inclusive, sustainable economic (GDP) growth, as a way forward to attain wellbeing and prosperity. (Rodrik 2017) Given the planetary plundering and overshooting its ecological boundaries, there is howver a real need to limit growth and organizing our societies and economies in a different way, including how we value labor, health, and care. Such a post-capitalist transformation requires paradigmatic change, principles and governance institutions. Given the ecological and climatecrises, or call it global risks (Beck 2006a) faced, societies are, urgently, forced to morally and politically imagine 
new ways of governing themselves on an overheated planet that faces a real risk of increased violence and conflicts. I argue that "this path is deeply political and risky, and requires engagement in societal debates and movements". (Van de Pas 2017) The last part of the thesis will provide for such a cosmopolitan outlook and what this would imply from an HWF perspective. Before doing so, I need to reflect on the relevance of our framework and how the research methodology has been applied throughout this thesis.

\subsection{REFLECTIONS ON CONCEPTUAL FRAMEWORK AND METHODOLOGY}

The overall framework has been constructed based on a 'Mind Map', originally made in 2015. This Mind Map was made to envisage the complexity of HWF development across different sectors and disciplines including its governance within, between, and beyond the nation states as the latter remain the most legitimate political institution to develop the HWF. (George et al. 2017) When looking at the intra-, trans-, and supraelements of global HWF governance, Kickbusch's framework on GHG along three political spaces seemed most suitable to capture those complexities and overlap in governance functions. I realized well that global health and its governance can be approached from different perspectives or so-called global health 'frames'. Cosmopolitan ethics based on equity and/or human rights considerations is one of them; security, public health, or economic development are other framings or even paradigms by themself. I understand the 'GHG along three political spaces' framework to 'bridge' these different frames. However, my experience in the domain made me aware of the inherent tensions moving the different perspectives forward. Rushton and Williams deconstruct the different elements of and provide a framework on how to analyze global health policy making. (Rushton and Williams 2012) In their approach, they clarify that policy debates are characterized by contestation between competing framings. These 'framings' are built upon broader 'paradigms of global health' and the 'power and authority' (agency) of the framer. Most importantly, and different from other global health policy frameworks, they also identify that behind the power, frames, and policy context, there is a 'deep core'. A problem "with many of the constructivist approaches to GHG is the over-emphasis on agency and the neglect of deeper structural determinants. The "playing field" on which global policy debates are played out is not level, but is skewed by historically specific and deeply embedded ideas and configurations of power." (Rushton and Williams 2012) The authors hypothesize that neoliberalism constitutes a 'deep core' of current global health policy making, characterized by privileging of market-based policy responses, commodification, liberalization of healthcare, and the individualization of risk and responsibility for health. Following Cerny, they state that embedded neoliberalism has become the 'hegemonic paradigm' during the last three decades. (Rushton and Williams 2012) When I reflected about 
the deep core of neoliberalism driving global health policy, I was inspired by Rodrik's Globalization Paradox, as its 'Political Trilemma of the World Economy' embodies well how hyper-globalization (deep economic integration) and its 'golden straightjacket', a kind of embedded neoliberalism, captures the democratic political space at national as well as the international level. (Rodrik 201la, p. 115) I have then considered this political-economy analytical framework to be a proper anti-thesis informing how global health policy is functioning rather than how it should be: the GHG framework along three political spaces. I combined the two and explored the tensions and dialectics between them in relation to a global health policy issue (HWF development), thereby exploring the different powers and authorities, policy debates, paradigms, and 'deep core' (hyper-globalization). Given that the debate on GHG in relation to HWF development is less developed than other GHG domains, say health financing or health security, I needed to see how to fit the several HWF elements coherently in the three political spaces at the national level; governance by the international health organizations and impact of policies outside the health domain. Interestingly, in HWF policy debates there seems to be a division between the governance of local and national HWF issues versus the debate on international HWF mobility. While this is, in reality, a kind of continuum, its governance spaces and the different actors involved appear separated. There is limited academic literature in this GHG and HWF domain, with the notable exception of the excellent overview work "International Health Worker Migration and Recruitment: Global Governance, Politics and Policy". (Yeates and Pillinger 2019) I had hence to construct my own conceptual approach, which has led to 'The Political Trilemma in global Health Workforce Governance' (Figure 1.3., Chapter 1). The three political spaces have formed the basis for the structure of chapters 3, 4, and 5. Chapter 6 provides study and analyses of what the space and potential are for current GHG, specifically for HWF development and labor migration. Hyper-globalization and neoliberalism can be considered a deep core and structural driver in this trend. Chapter 7 is a discussion of these separate spaces and GHG directions, applying a politicaleconomy perspective and reflection on the HWF development space in the political trilemma. The Figure in chapter 7 (Figure 7.1) provides a graphical overview of these tensions playing out in Global HWF governance. Notably, but not surprisingly, is that there is pressure on democratic politics and that critical remarks can be made about the legitimacy of several global HWF developments, especially in relation to who is represented and how deliberation is organized.

As I have followed a political-economy lens assessing global HWF policy issues, I have either neglected or complemented other approaches that analyze the mezzo- and micro-level of health systems governance. My approach has specifically tried to capture global HWF governance pathways at the macro-level, with the exception of the Guinean case study. Moreover, one could assess HWF policy also from another 
disciplinary angle, e.g. it being a labor economics, public health, innovation, humanitarian, human rights approach. I would like to ask for caution that my chosen conceptual approach is considered the 'right' one and others being 'wrong'. Other analytical approaches can be considered complementary and deserve dialectic, interdisciplinary approaches in academia, policy, and politics to address the global HWF challenge. I would like to hereby acknowledge HWF conceptual work that I might have referred to, but not specifically used. It must be said, however, that my framework, by taking an explicit health equity and the political-economy lens starts from the normative perspective that hyper-globalization and neoliberalism are core problems rather than enablers for just HWF development. My 'grand' theoretical perspective might hence be different from other HWF conceptions.

In a recent overview article by Cometto and colleagues on how to develop the HWF for UHC, the authors take the 'HRH Action Framework' as analytical basis. This public health framework looks specifically at elements of leadership, finance, policy, education, partnership, and human resources management systems. It is an excellent approach on how to improve HWF functions at the national level. The authors acknowledge: "Other factors exist outside the control of policy-makers in the health sector, which in turn have a fundamental role in determining the political, technical and financial feasibility and sustainability of health workforce policies and actions. While recognizing their importance, these factors fall outside the scope of this paper." (Cometto et al. 2019) This thesis is complementary to the HRH action workforce framework by analyzing these factors specifically.

The HPSR reader on HRH published by the WHO includes another framework used in HWF studies. This reader assesses the HWF more from a micro- and mezzo-level, within countries. Economic, political, and societal contexts are considered but mostly at the domestic level. (George et al. 2017) This health policy and systems approach is useful and I have applied elements of it in the two studies in chapter 3, focusing and comparing HWF development at the national level. Also, it is not surprising that the editors have restrained themselves to analyzing the topic domestically as this is within the technical mandate of the wHO and indeed nation states remain the main constituency to develop HWF policy. This thesis nevertheless clearly indicates that hyper-globalization does interfere with this national policy space. This 'externality' is too often neglected in Health Policy and Systems Research (HPSR). Another, muchsupported approach by the wHO, is the health labor market analysis and its policy levers. (Sousa et al. 2013) I have referred to this framework for the study on HRH policy development in Guinea, although this was a policy analysis rather than a labor market analysis. The health labor market framework is a useful tool but likewise, it is positivist in understanding what should be done, rather than including and accounting for (economic) externalities, agency, and power issues. Having said that, 
there are studies that use a specific political-economy lens and health labor market approach for analyzing the HWF. (Fieno et al. 2016) The main study in chapter 6 applies a human rights analysis to GHG. Interestingly, there is less focus on human rights in HWF development, vis-à-vis other global health domains such as UHC and the IHR. For excellent work on GHG, UHC and the Right to Health, the conceptual work on global constitutionalism by Ooms and Hammonds may be referred. (Ooms and Hammonds 2016) I have not delved further in the rights-based approach to HWF development, partly as I see limitations and enforceability of international human rights frameworks. Human rights scholars are better positioned to assess possibilities and constraints. An overview publication on HRH global governance and international migration is a unique exception by linking the several governance domains from a rights-based approach. (Yeates and Pillinger 2019) I have not explored in detail labor migration theories, which could have provided an additional perspective on the study in chapter 4 that explicitly looks into HWF migration and its governance. (Bailey 2001) The study in chapter 5 builds on what is known as the Copenhagen School of Security Studies. The concept of 'securitization' beyond traditional security actors is critically analyzed in International Relations. (Buzan et al. 1998) It is applied to global health security concepts. The securitization of health policy and programs is widely researched, especially in relation to issues of rapid response, surveillance, vaccine-development, AMR. Health personnel, and their agency, is an important, but so far underrepresented actor in securitization research. (Elbe 2012) There is a lot of attention to the need for innovation and digitalization in the HWF and its practice. Britnell takes a contrasting perspective to me in overview work on how to solve the global workforce crisis in health care. "I firmly believe that humankind is capable of solving the global workforce crisis with the help of the technology it has created." (Britnell 2019) He suggests orchestrating ten large-scale changes. In essence, these recommendations are about reframing the HWF issues to one about productivity, and wealth creation; governments to be entrepreneurial; provide new models of care and make patients 'active partners in care'; recognize informal care and create a new cadre of care assistance; and stimulate disruptive possibilities offered by Artificial Intelligence and robotics and embrace these new techniques. (Britnell 2019, p. 2) Clearly, Britnell, who has been working as a manager and consultant for over 30 years on addressing HWF challenge, has a different normative and theoretical perspective on how to solve the HWF crisis. In his view, hyper-globalization, technical innovation, entrepreneurialism and individualizing responsibility are enablers to overcome the problems that we now have. While his conceptions are diametrically different from mine, it would be relevant to have a dialectic exchange between the two of us to see where precisely contestation and possible overlap exists.

I have only taken limited attention to worldwide developments in developing the community health workforce (CHWF) and the need to take a gendered-approach 
to HWF development. While reflecting on this, it is telling that from a politicaleconomic perspective these constituencies are under-represented in governance considerations. 'Informal' care is still undervalued. Women carry most of this burden. Kindly refer to a recent publication by the wHO for conceptualization on how to strengthen the HWF from a gendered angle. (World Health Organization 2019a) Likewise, there is now more systematic evidence on how to integrate and govern CHW in health systems (Scott et al. 2018) From my perspective, however, is there still too little attention to, and research on, the political-economy and inequities related to the deployment of CHW. CHW programs might be a charitable approach in addressing essential health needs whilst from a rights-based approach and social contract theory people would be entitled accessibility to formally-trained health personnel. There remain important questions about whether cHW programs improve or sustain health inequities. (McCollum et al. 2016) Lastly, complex systems thinking, adaptive governance modalities and realist evaluation methodologies are frequently applied in assessing the functioning of health systems and the workforce. Often, this is done at the organizational and mezzo-level. (Prashanth et al. 2014) I have not used these middle-range theoretical frameworks, which in essence combine empirical with theoretical approaches, for analyzing global HWF development. I could have potentially done this in the Guinean HWF policy study and I would not exclude it in future research. Although there is much attention to complex adaptive systems in global governance and global health (Held et al. 2019; Hill 2011; van Belle et al. 2018), I have decided not to focus on these frameworks. I have reflected on this earlier but one of the main reasons is that these frameworks are less suited to analyze frames, power, processes, and agency in global health policy. Moreover, rather than relying on a middle-range theory as the main framework, I choose to focus on a grand-theory: the Political Trilemma in relation to GHG. However, Beck's cosmopolitan outlook could also be regarded as such a middle-range theoretical approach and while reflecting on this I realize that there is potential contestation between the political trilemma and GHG models that I have applied as overarching frameworks, and Beck's methodological cosmopolitanism. Let me explain this in the section on methodological limitations below.

\subsubsection{Boundary spanning, multi-perspectivism and epistemic limitations}

I have approached the Global HWF problem as a complex challenge. I have aimed to apply 'a boundary transcending and boundary-effacing multiperspectivism', following Beck's methodological cosmopolitanism. (Beck 2006a, p. 82) I have aimed to do so by looking into the three different political spheres and their overall outlook from slightly different perspectives. The studies in chapter 3 used a health policy analysis (policy triangle) methodology, more frequently used in HPSR. The studies in chapter 4 , especially on the wHO reform, applied a political science framework 
on legitimacy. The study in chapter 5 followed critical security studies concepts on securitization and the Foucauldian notion of biopolitics. The main study on GHG in the SDG used a human-rights analysis as the main entry point. In the discussion, I have focused on the three spaces by applying a political-economy analysis, Rodrik's political trilemma, on the three global HWF governance spaces and overall directions. With regard to using multiple perspectives, the cosmopolitan outlook worked out well. Nevertheless, one can question how I have analyzed the HWF phenomenon "both locally and nationally and transnationally and translocally and globally”. (Beck 2006a, p. 82) Following Brown and Labonté, this could be best done by analyzing globalization in a transformationalist matter, as the phenomenon is a pluralist one. This would require a multidimensional approach not only involving macro-economic approaches but also micro-level qualitative processes to capture processes of 'glocalization'. This includes a more inductive observational approach in addition to the more deductive theoretical conceptualization. (Brown and Labonté 2011) When reflecting now on the studies and methodology of this thesis, I should ideally have included more attention to the 'local'. The study that covers most of this local perspective is the HWF analyses in Guinea. This study is nevertheless not observational but focuses, via conducting semi-structured interviews on local policy and governance issues, on the retention of health personnel in rural districts. I have reflected on this with Guinean colleagues. A second study published is also policy-oriented (Kolie et al. 2019). Whilst not-yet-published new workforce data from Guinea are comparative and epidemiological in character. Nevertheless, we have planned to conduct a socio-anthropological study on a phenomenon that is called 'waithood'. Waithood is a situation in which stable jobs disappear and young people cannot support themselves and their families anymore. Most young Africans are living in a period of suspension between childhood and adulthood. (Honwana 2014) The idea is to analyze this phenomenon from the viewpoint of young health professionals in Guinea. These have little perspectives on a stable job in the health care sector. Here we aim to understand their adaptive strategies, labor mobility as well as possible migration pathways. This is an excellent research to be conducted with my Guinean colleagues being in the lead. However, I myself would have ethical considerations conducting such a 'local' research. This relates to what has been described as the 'foreign gaze' in global health. (Abimbola 2019) This is a form of authorship whereby the 'foreigner' publishes his research in a 'foreign' expert journal, often in English, recognized by fellow academics and practitioners. The question is whether the production of such local knowledge would contribute to transformational and meaningful change? Would it not be 'ideal' to have local experts publish in local journals? (Abimbola 2019) The author does not exclude that the two could be complementary. It nevertheless raises important ethical questions about what is ' $(\mathrm{g})$ local' research and how authorship and partnerships in global health is constructed. (Chu et al. 2014) I would agree that '(g)local' in this regard indeed also implies my 
own place and environment. I have recently published research with colleagues, in the form of a policy brief, on the solidarity principle and situation of HWF mobility in the European setting. (Mans et al. 2020) Such an approach to local global health research is relevant to overcome the foreign gaze and 'othering' (Said 2014) which remains pervasive in global health teaching and research.

Besides a relative lack of the 'local' in this thesis, I also encountered another challenge. As I methodologically conceptualized to study the three different political spaces of global HWF governance action, I needed to ensure that there is logical coherence between the different spaces, a form of boundaries; hence the chapters of this thesis. I have done this conceptually through a 'boundary-spanning' approach which is described as the practices of "reaching across borders, margins, or sections to build relationships, interconnections and inter-dependencies in order to manage complex problems therefore of relevance to the development of Global Health thinking." (Sheikh et al. 2016) Boundary spanning involves a mindset for learning, one that goes upstream, downstream, and one that goes laterally. My methodology is sufficiently downstream (deductive) and laterally (comparative) oriented, but could benefit from an upstream (inductive) case study. Nevertheless, I hope that my approach contributes to the aim that that "effective boundary-spanning can also be contingent on questioning one's own power and privileges... it requires a mindset of inclusiveness, awareness of and respect for different coexisting realities, which is often missing in current Global Health thinking and action." (Sheikh et al. 2016)

There have also been the practicalities and limitations of studying these different spheres during the research method. As outlined in chapter 2, I have conducted these studies, not as part of one integrated and financed research project. Rather, I collected the data, and integrated them according to the constructed framework, over time. The studies involved in the several chapters were financed through public funding by at least four different agencies via four different research and policy programs. This is done in an academic global health landscape "marked by chronic underfunding of universities and reliance on soft-funding through research projects that are short term and donor prioritised". (Storeng and Palmer 2019) To be clear, I have not experienced censorship in my research during this thesis period. There is nevertheless a tendency in research funded through bilateral and multilateral development agencies, which has been the case in this thesis, for 'rose-colored' reporting. This so-called "success cartel in Global Health" (Rajkotia 2018) may lead to an over-attribution of country governments or institutions in reaching global health goals; in my case HWF development. If one reads carefully through the different studies in the chapters, then one can distinguish a slightly different tone of voice between the funded studies in chapter 3 , 4, and 6 , and the overall analysis in the discussion in chapter 7 . For these separately funded studies (Guinea HWF development, the WHO policy tracing HRH commitments, analyzing the 
Global code relevance and efficiency, Global health governance in the SDGs) the tone of voice is one of 'the glass being half-full'; There are challenges, but with careful reflection and adaptation, we can overcome them. The overall discussion chapter and analysis is more critical, looking at the 'glass being half-empty', analyzing a gridlock and identifying structural challenges in overcoming the political economy of and power inequalities in global HWF development. This criticism was already present in chapters 5 and 6 . What is remarkable is that depending on the different epistemology applied, these representations, the glass half-full and half-empty, are both analytically correct. It depends on the perspective as well as on the presentation and framing of the results and its implications for policymakers and funders. In my own research and writing, I try and not escape my own normative position of 'framing' and 'construction' of a policy perspective that is the subject of my study. Regardless, rather than the 'either/or' dichotomy of the first modernity, Beck invites us to take a second modernity approach, being reflective and recognizing that there may be an 'and/and' outlook, a multi-perspective approach in methodological cosmopolitanism. (Beck 2006a, pp. 82-83)

Reflecting on the boundary-spanning approach, it is noteworthy that in essence, all the funded studies structured my research to remain largely within the 'boundaries'. These boundaries include the nation state and its sovereignty as the analytical 'denominator' while comparing the country's health systems and its economies, as is so often done by multilateral agencies such as the wHO and the wB. There is however some deep artificiality and ontological incorrectness in comparing nation states, their systems, and societies. Are these nation states not all unique, within their creation, historical path dependencies, and socio-political settings? "Social structures and processes are becoming cosmopolitan, whereas scientific knowledge remains beholden to the axiomatics of the national". (Beck 2006a, pp. 85-92) I felt this 'axiomatic of the national boundary', most strongly in the studies in chapters 3 and 4 . This is also not strange as these were done at a country level, through bilateral international cooperation in Guinea as well as through multilateral international cooperation via the wHO. Despite framed as 'global health' programs, this policy and research cooperation on health often remains inter-national, hence between nation states with clear set boundaries and physical borders. The containment of public health research within such a boundary has then the effect that it hinders a cosmopolitan outlook. This has a side-effect that certain political-economic and power-related problems in unjust, hyper-globalized, economies and labor markets, as well as their representations, become 'silenced'. (Bacchi 2012) Besides national boundaries, I also faced disciplinary boundaries and difficulty creating a dialectic in relation to health labor market discussions. I am not an economist, so am I aware of the right disciplinary methodologies, epistemics, and boundaries? I encountered these comments in peer-reviewed comments on the Guinean HWF study as well as in the 
'HWF agenda beyond economic growth' analysis. It is hence encouraging to read that a group of health economists have currently undertaken a systematic review on 'the impact of economic recessions on healthcare workers and their crises' responses'. (Jesus et al. 2019). This research aim is complementary to but in line what has been analysed in this thesis.

In addition to the disciplinary boundary, there is also the sectoral boundary issue. Working for a public health advocacy NGO, as I still did while engaging with the wHO processes and policy debates in chapter 4 , provides a different positionality than being an academic researcher. The study in chapter 5 on the AHCG builds on my own experience working for a humanitarian NGO. Both NGO and academic positions generate a different legitimacy via their epistemics. The first position allows for an inherent 'normative' position but it must be clear that this is an 'advocacy' position. The second position provides an evidence-based, methodologicallysound, scientific analysis, but this is then considered 'objective' and academically independent from institutions and contexts. The tension between these two has been aptly described by the need to 'foster a better dialogue between empirical and normative disciplines'. (Ooms 2014) In résumé, within the seperate studies outlined in the chapters, because of funding and settings, I was more or less obliged to remain within the epistemic boundaries. It has been only possible in the discussion of this thesis, through applying methodological cosmopolitanism and analyzing the global HWF governance complex (thesis) across three political spaces, within its political trilemma (anti-thesis), and from multiple perspectives, that I have been able to be boundary-spanning, at least partially, as I will now conclude.

This thesis has been able to capture multiple dimensions, across spheres, boundaries and capturing complexities. Nevertheless, it could include more inductively oriented, contextualized driven research, albeit the research from Guinea being included. Reflecting on the methodologies of analyzing the globalization and health nexus (Garrett W Brown and Labonté 2011), I realize that my research provides more of a skeptical- rather than a transformationaloutlook on the impact of (hyper-) globalization, GHG and its effects on the HWF development. This could have two potential explanations. The first explanation is that the methodology, focusing on policy and governance matters, might not have been differentiating enough to capture (realistic) cosmopolitanism trends in HWF mobility and migration. (Khan et al. 2015) The second explanation, and this is more likely, is that the applied framework of GHG and the political trilemma provided a 'grand' theory and outlook. This political-economy analysis of analyzing the role of (hyper-) globalization was more skeptical from the onset, given its focus on economic global straightjackets. (Friedman 2000) However, Rodrik's political trilemma allows for differentiation and it is not set in stone. There are pathways and 
policy recommendations to pursue a more sane form of (economic) globalization. (Rodrik 201la; 2017) These would include regulation and a traffic light system in relation to international trade, financing, and taxation. There would also need to be a shared-sovereignty approach between states in promoting global public goods as well as dealing with global public bads. Nevertheless and despite a global financial crisis, have both policy options not been (really) pursued by the major economies during the last decade, including those in relation to health systems development and health labor migration. Although there is a cosmopolitanism trend in the education, employment, and mobility of the workforce, there is currently limited space for a cosmopolitan outlook on HFW governance development. HWF employment and health systems development remain domestic responsibilities for nation states, while the international migration of health workers is governed by ethical codes, bilateral cooperation, and adaptive global governance arrangements that provide only limited guarantees and rights for the migrants themselves as well as the sustainability of health systems in the country of origin. With this rather sobering message that the cosmopolitan outlook on HWF development is truly difficult in the current political climate, I would like to introduce the last part of the discussion and thesis. There might actually be a cosmopolitan outlook to global HWF development and mobility but this requires socio-political as well as scientific paradigm shifts. It would require us to consider work, care, and wellbeing beyond economic growth. I provide an outline as to what this could look like.

\subsection{A COSMOPOLITAN OUTLOOK ON HEALTH WORKFORGE DEVELOPMENT}

Within the current gridlock of economic hyper-globalization and with countries retreating from multilateralism, the outlook to cosmopolitan policy-making and approaches for HWF development may be grim. Nevertheless, I have provided in the two analyses included in chapter 6 a basic outline for pathways and momentum that seem to emerge. Such pathways would move beyond capitalism as the 'core' driver of international cooperation. To clarify, these pathways would also move beyond the 'boundaries' of our GHG and political trilemma framework as both Rodrik and Kickbusch acknowledge in policy proposals that sustainable and inclusive economic growth, as envisaged within the SDG, is required for development objectives, including health. Before I explore post-capitalism pathways, let me first return to the cosmopolitan outlook and cosmopolitanism as social and ethical principles in the second decade of the $21^{\text {st }}$ century. Beck writes that a radicalized modernization has undermined the first modernity by five interlinked processes: globalization, individualization, gender revolution, underemployment, and global risks. He calls for a paradigm shift, a frame of reference (what he calls a second modernity) or a pluralization of modernity. (Beck 2010, pp. 217-28) Beck 
recognizes the underemployment and flexibility of labor, the emergence of several interconnected global risks and the decline in power and legitimacy of governments. He thinks that the global free-market ideology is collapsing and that there will be a politicization of the global market economy. He identifies 'a new cosmopolitanism', in which a political economy of uncertainty, of a world risk society, might be developed. This new cosmopolitanism is to be based on something like a cooperative or altruistic individualism. In times when increases in per-capita GDP leads to little or no employment growth it is of relevance to connect, a sense coming from fragility and risks, by means of cultural democratization and political freedom. Beck argues that to be able to be politically free one should not have the insecurity of basic daily needs required for oneself and family. To get there, Beck argues for a "basic assured income as a sine qua non for a political republic of individuals who will create a sense of cohesion and fellow-feeling" (Beck 2010, p. 224) While reflecting on Marx's Communist manifesto, Beck argues for a Cosmopolitan manifesto. The first one was about class, the latter is about the transnational-national conflict and must be about dialogue; the possibility of democracy in a global age. He is positive about the several transnational movements (ecological, feminist), institutions and universities emerging, focusing on a post-national understanding of politics and responsibility. Beck promotes a 'world citizenship' (Kant) but provides a warning: “A distinction must be drawn between 'global capitalists' and 'global citizens'. Yet, a plural world citizenship is soaring with the wind of global capital at its back. For the bourgeois must already learn to operate in his or her own interests in a transnational framework, while the citizen must still think and act within the categories of the national state." (Beck 2010, p. 228)

It would be interesting to conceptualize what this 'new cosmopolitan' thinking then would imply for an HWF being part of a 'global citizenship' constituency. Interestingly, it is not only philosophers and sociologists imagining such a new world order. There is also a trend within the economics discipline aiming to reform thoroughly how economic theory and practice contribute to a 'safe and just space for humanity'. This need for change has been described most aptly by Kate Raworth in her publication 'Doughnut Economics'. (Raworth 2017a) Her 'economic theory for the $21^{\text {st }}$ century' is based on the planetary boundaries framework. (Steffen et al. 2015) This planetary boundaries framework has analyzed the status of nine ecological, earth-system processes and defined for those processes the so-called planetary boundaries-this being understood as the 'safe operating space' for human societies. "Respecting these boundaries would greatly reduce the risk that anthropogenic activities could inadvertently drive the Earth system to a much less hospitable state."(Steffen et al. 2015) This aggregate research indicates that for two out of nine planetary boundaries, there is already a high risk to destabilize planetary integrity as these boundaries have been transgressed. This pertains to the boundaries of biosphere integrity (extinction rate of biological species) and biochemical flows (notably nitrogen). This framework also indicates an 
increased risk for planetary integrity in relation to climate change, where there is a boundary overshoot. (Steffen et al. 2015) The innovation that Raworth provides is that she outlines the economic principles not only to respect the planetary boundaries but also to ensure that the social foundations for humanity are being met. She calls this a $21^{\text {st }}$-century compass for a safe and just space for humanity and has called this "the Doughnut", given the shape of the framework. (Raworth 2017a, p. 44) The outer ring of the doughnut represents the planetary boundaries; the inner ring, the social foundation and is based on the 12 basics required for life. Health and income/work are two of those basics. The Doughnut framework, including its shortfalls in social foundations and overshoots in planetary boundaries, is depicted in its entirety in Figure 7.2.

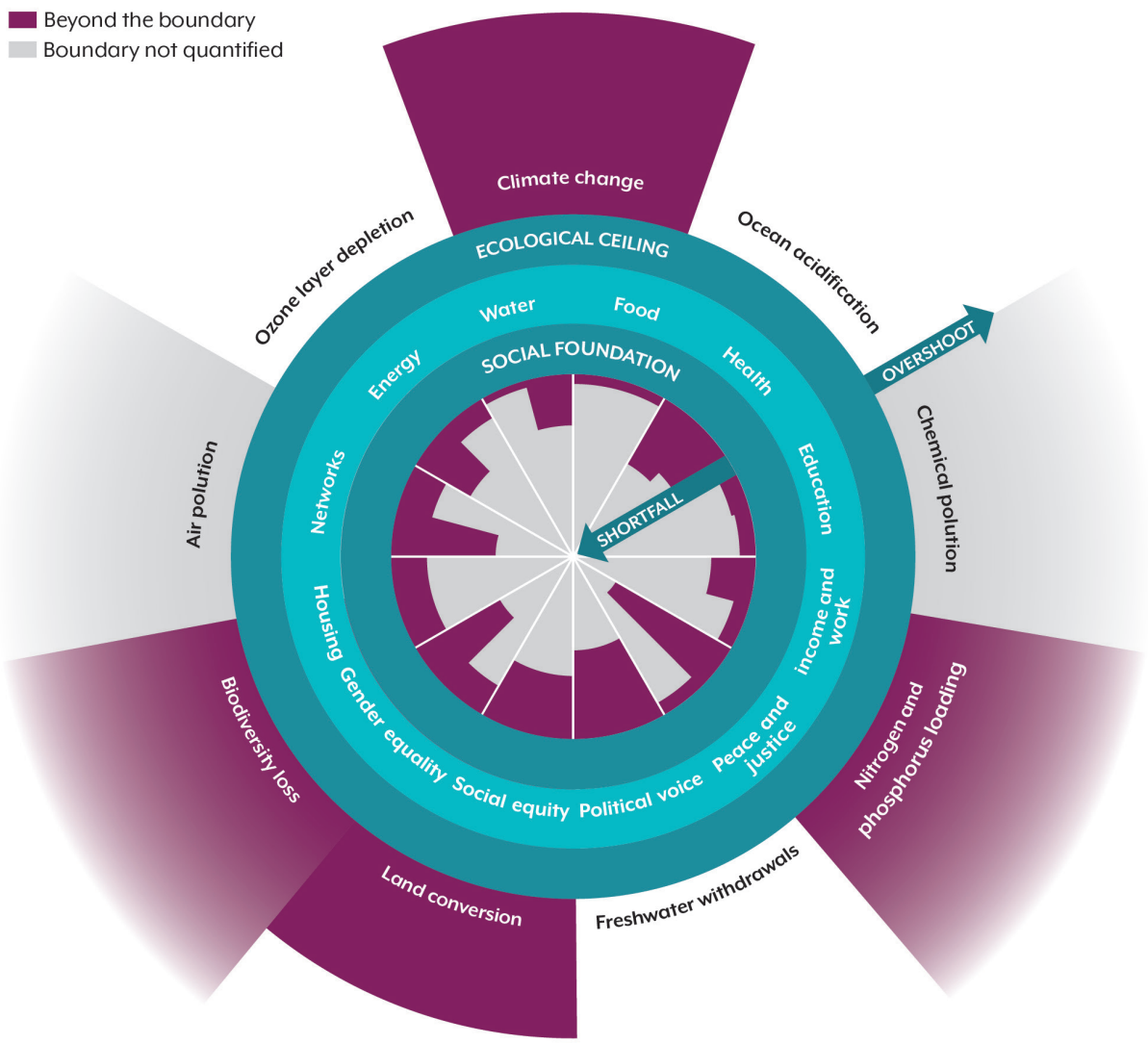

Figure 7.2: Shortfalls and overshoots in the Doughnut

Dark turquoise circles show the social foundation and ecological ceiling, encompassing a safe and just space for humanity. Purple wedges show shortfalls in the social foundation or overshoot of the ecological ceiling. (Kate Raworth 2017b) 
In essence, Raworth takes forward thinking and critique on economic growth principles that were put forward by the Club of Rome ${ }^{1}$ in 1968 and its landmark publication 'Limits to Growth'. (Meadows et al. 1972) Raworth stresses on the different economic thinking and practice required in the $21^{\text {st }}$ century as to stay within the doughnut. She identifies seven economic principles for this: 1. The Doughnut instead of GDP as the main goal; 2. Embedded economies; 3. Social adaptable humans instead of the rational economic man; 4. Allowing dynamic complexity; 5. Economics that are distributive by design; 6 . Economics that are regenerative by design; and 7. Economics that become growth agnostic instead of growth addicted. (Raworth 2017a, pp. 25-30) "We need an economy that makes us thrive, whether or not it grows.” (Raworth 2017a, p. 268)

From this 'new' circular-economy perspective, it would be relevant to understand the role of the HWF. Although Raworth is not specific about this, she provides some hints that are relevant for health, employment, and well-being. Firstly, she outlines the fallacy of the 'rational economic man'. People are dependent on, and caring of, each other and not as calculative as an older behavioral economic theory makes us believe. Health care workers have an intrinsic motivation to do their work and monetary incentives may trigger extrinsic motivation rather than reinforcing these intrinsic values. This is to say, if health care workers do receive a decent, sufficient, living wage then they will do their job with passion and competency. (Raworth 2017a, p. 117) Performance-based financial incentives will not improve productivity or efficiency in the health services. (Paul et al. 2018) Moreover, she warns about the creation of market mechanisms "into spheres of life traditionally governed by nonmarket norms... and sometimes, market values crowd-out nonmarket norms worth caring about." (Raworth 2017a, pp. 120-21) This is an indirect critique of the health labor market approach and its use of 'rational' policy levers and regulations to ensure that people behave according to capitalist logic. This contradiction in market-based capitalism and its relation to the 'crisis of care' is explained in detail by Fraser. She clarifies how the realm of social reproduction and care has become rapaciously subjugated to production under financialized-capitalism (Neoliberalism 3.0). She calls, like Raworth, for structural transformation of the capitalist social order, but without sacrificing emancipation or social protection. (Fraser 2016) This vision hence contradicts the rationale by the wHO and the UN to invest in the workforce to drive employment and economic growth (an increase of production and consumption by individuals and societies). This is also my argument in chapter 6 where I suggest expanding the HWF policy agenda beyond economic growth principles. (Van de Pas et al. 2018) Secondly, Raworth also makes the case that if we want our economies to thrive, then we should be able to finance universal public services like health

1 https://www.clubofrome.org/. 
care. Our economies need to be redistributive by design, to enable people to create capabilities, including being healthy. (Nussbaum 2011) At this moment in time, there should not merely be a redistribution of income at the national-, but moreover a redistribution of wealth at the global level. Raworth is critical about the role of ODA and mentions that labor migration, including in health care, is actually one of the most effective ways of reducing global income inequalities. This nevertheless depends on whether income inequalities in the host country reduce, as precarious working conditions could hinder community connections and social integration of migrants. Raworth, like other major economists, calls for taxing extreme personal wealth, closing tax loopholes and havens (the Netherlands being one of them), developing a global financial transaction tax, and/or a global carbon tax. (Raworth 2017a, pp. 198-205) The proposals are not new and have been elaborated in detail by others. They do challenge existing power and capitalist accumulation and would probably require a political 'shock momentum', likely a major crisis. Lastly, Raworth urges us to focus on economics beyond growth. Actually, economic growth should not be the overall aim but economies should rather, whether growing or contracting, support societies and ecology to thrive. As part of her argument to become growth agnostic, she refers to the original and revised 'Limits to Growth' models. GDP growth has largely happened due to the availability of cheap fossil fuels. "Future GDP growth is not only not guaranteed, it is more than likely to end within a few decades." (Raworth 2017a, p. 263) The thing is: households, communities, and societies might even exchange and value services (like health care), food, and goods without monetizing it implying without no money changing hands. This is the true collective power that citizens and societies have. Or "If there are indeed limits to growth, then the political underpinnings of our world fall apart." (Raworth 2017a, p. 266)

These underpinnings can be seen in the political trilemma framework and its elements used in this thesis: being political power (the nation state and its controlling agencies), accumulated capital (hyper-globalization) and labor unions; social and citizen movements, political parties (democratic politics). While we concluded that there is still a kind of 'gridlock' in the political trilemma, Raworth assumes that this capitalist construct will fall apart at one moment. Rather than labor and societies to be adaptive and resilient in capitalist economies, it is likely that there will be implosion and transformation. This will nevertheless not go without conflict and intense distributional conflicts will re-emerge within and among countries. (Raworth 2017a, p. 266) Indeed, the outburst of social uprisings and protest movements in countries around the world in 2019 indicates that we are going through such a transformation and that we are currently at a stage of late-capitalism. Nevertheless, countries that aim to sustain GDP growth in an economy that may be close to maturing, and I would argue that most Western European economies are, may take destructive measures. These include deregulating finance, privatize public services like health care, and 
adding into national GDP accounts things like 'ecosystem services', 'national capital' and then also 'human capital', like health care. (Stein and Sridhar 2019) "These policy choices are akin to throwing precious cargo off a plane that is running out of fuel." (Raworth 2017a, p. 269) Raworth also provides some policy advice on how to move to a growthagnostic, post-capitalist, economic order. I would adhere to most of them, and what they would eventually imply for health systems functioning. Firstly, moving forward with tax justice initiatives and public investment along the lines mentioned above. Secondly, shorten working-weeks so as to tackle both unemployment and overwork, while ensuring that people have still a decent and sufficient living wage. The standard paid working-week in Europe ought to move from above 35 hours to just 21 hours. Thirdly, civil society movements and networks like the C40 network (an alliance of the world's megacities) might provide alternatives to the powers in the G20 alliance of major economies. For instance, Amsterdam is at the moment in a process to organize its urban economy around the doughnut model. (De Walle 2019) However; "the compulsion of the old GDP game holds its grip because GDP brings both global market and military power." (K. Raworth 2017a, p. 280) Lastly, individuals and societies, especially in HICs need to learn to step away from the treadmill of consumerism, addiction to GDP growth and rather live by the principle of sufficiency instead. What really matters for us in life includes using our talents for helping others and standing up for what we believe in. (K. Raworth 2017a, pp. 280-81) Interestingly, the health care sector could play an important role there as the human sense of connection, giving, supporting, and developing skills matter to most health care workers and patients alike. Indeed, in my own professional practice, I have also experienced that these intrinsic drivers mattered for my colleagues and me. Having practiced in a social psychiatry department in the Netherlands, Primary Health Care in post-conflict El Salvador, a refugee health clinic in Darfur, Sudan, and HIV/ AIDS care in West-Papua, Indonesia, I have experienced that there are universal and intrinsic drivers that make health care workers commit to their caring role, mutual trust and responsibilities. However, contextual factors, socio-political or other insecurity, financial-, organizational, and systematic-challenges make it difficult for individuals to maintain such commitment and pursue their work in a sustainable manner. Perhaps, the real cosmopolitan realization is that the majority of health care workers around the world are not primarily rational economic men/women, a 'homo economicus'. Instead, the majority of them are nurturing, caring humans, the 'homo socialis'.

There are several thinkers, practitioners, movements, cities, and even countries (e.g. Iceland, New-Zealand) preparing for a post-capitalist society, supporting the global commons, while respecting planetary boundaries. The question will be how 'cosmopolitan' these new powers and constituencies will be? Many LICs still require a form of economic growth, an expansion of the domestic industry, to generate 
a fiscal basis to create jobs in the health sector. What will be the solidarity and shared responsibility provided by these new post-capitalist entities? Will there be a cosmopolitan form of financial redistribution allowing for the generation of millions of jobs in the health care sector? Or will we see new forms of global injustices, by some already indicated with the term 'eco-apartheid'? (Cohen 2019) To stay within Beck's terminology: will there be a cosmopolitan political momentum that a global capitalist order (societé bourgeousie) will be complemented or even replaced by a global citizen order (societé citoyenne)? What will be the role, and agency of the HWF at local, national, transnational, and supranational levels to contribute to such a transformational change? These are relevant questions; not only from an academic perspective but even more in a practical and socio-political manner.

Hickel, a scholar of development policy and Degrowth economics (Hickel 2019) and proponent of global economic redistribution also provides health and policy labor recommendations for a post-capitalist order. He argues for just wages integrated into a global system of labor standards and regulation. This would imply that wages either have to be above the national poverty line or at the threshold of $50 \%$ of the country median wage. This could be an approach as well for the wages of midlevel care workers (nurses, midwives). A global minimum wage and a global social protection floor could be an important basis to sustain the workforce in LMICs. (Hickel 2017, pp. 267-68) Like Raworth, Hickel urges to move beyond GDP growth. He has recently proposed a Sustainable Development Index (SDI) as an alternative to the Human Development Index, as the former would also include ecological considerations (the planetary boundaries). Cuba, Costa-Rica, and Sri Lanka rank first, second, and third within this SDI country list. These countries remain in safe limits of their national ecological footprint while at the same time providing decent education, employment, and health care. (Hickel 2020) Hickel also proposes a basic minimum income policy as a strategy for poverty reduction, even more as a possibility to improve working conditions and wages. It would also allow for basic security for citizens enabling them to provide informal, home-based care for relatives and other people with health needs in their social environment. (Hickel 2017, pp. 296-97) Other thinkers writing on post-capitalism policies like Mason and Bregman likewise write on the need for basic income schemes and a separate minimum wage as an enabler for social security, trust, and care within societies. It could also potentially allow for non-monetized, liquid exchange of services between citizens, e.g. a lawyer exchanging his services for daycare. (Bregman 2017; Mason 2016, pp. 284-86)

I must admit that in these transformative, macro-perspective analyses and proposals for cosmopolitan policy changes in times of a climate emergency, there is limited attention to the specific role that the health sector or health care workforce could have. It appears that the latter is still regarded within its local, organizational or 
at best, national context. When talking about global HWF issues, health personnel is quickly depicted as the 'foreign health migrant' while, of course, they embody complementary identities, being part of a 'transnational' place, diaspora, and globalized community. Not much has been published on Degrowth policies and their impact on health systems and its workforce. Zywert writes that it is likely that health systems could become more pluralistic and that they will contract due to a reduction of fiscal transfers. It could lead to a process of "re-embedding care in reemerging networks of family/community reciprocity." (Zywert and Quilley 2018) Perhaps reverse innovation might take place here as in LMICs pluralistic, albeit underfunded, adaptive health systems are the norm rather than the exception. (Crisp 2010) Missoni has written specifically on Degrowth economics and its impact on health care systems and builds on the Medical Nemesis thinking by Illich. I follow much of Missoni's analysis when he writes that 'localization' and lifestyle changes seem to inspire the dominant perspective of Degrowth supporters, in some cases explicitly excluding a possible role of global institutions. Post-growth alternatives and governance require supportive policies at the national and global levels. Missoni sees an essential role for civil society organizations. He proposes that "In a degrowth perspective, a strong alliance between WHO and a wider movement of CSOs bringing together scientists, practitioners, and activists who embody degrowth ideas in new material spaces, growing in a movement capable of building alliances with other similar cultural stories and movements, may represent a strategic step also to promote healthy policies in other domains... This will have to be pushed and probably literally reinvented through dynamics and mechanisms that will undoubtedly require further investigation and analysis." (Missoni 2015)

This leads me to my final conclusion. Chapters 3 to 6 of this thesis indicate that the cosmopolitan outlook to HWF governance and development had its limitations in the first modernity, under capitalist economic growth and hyper-globalization. A political momentum for cosmopolitan health cooperation may occur as economies will eventually, due to the ecological limitations come to a steadystate, or even contract. In such a Doughnut circular economy, or even economic Degrowth scenario, health care professionals may play a crucial role in society as they may provide value and care beyond the monetary value. They may be an important element of the social construct on which societies will still prosper in times of reduced material output. Actually, this crucial role is not new as health care workers have been central to the development of the modern state since the $19^{\text {th }}$ century. (Foucault 2002) However, health care workers, being likewise part of the cosmopolitan second modernity, have the potential agency to contribute to, or reject upcoming transformative changes in our health systems, economies, and societies. Likewise, they will do both, and there may be dialectics and conflict about these roles, as the HWF is far from a politically homogenous group. The question will be how cosmopolitan the outlook of the HWF will be beyond clinic 
and place; and whether they will be able to develop a transnational professional ethic beyond the 'here' and 'there'? Education, exchange, and narratives play an important role here.

I realize that while writing these final words of this thesis that there has been limited space for, or representation by, the perspective of health workers themselves. Of course, I am still, and will remain, a health professional myself. And yes, I have spoken with several health workers in the studies, albeit them being more in a policy, management, or governance capacity. An inductive understanding of how the HWF, in multiple places, engages with rapid economic and ecological changes under globalization has to be included in the follow-up work of this thesis. Nevertheless, and to paraphrase Missoni's words, this local and national engagement has to be complemented by a transnational HFw movement at the global level. There are considerable strong networks, organizations and experiences existing ${ }^{2}$ on which a new generation of health professionals can build while contributing to climate and health justice. I would like to end this long piece of work, sweat and tears with the words of Eduardo Galeano: "I advance two steps, it goes two steps backward. I take ten steps and the horizon moves ten steps forward. No matter how far I walk, I will never reach it. What is the use of utopia? That's its use: to help us walk." (Galeano 1993)

2 Including, but not exclusive to, networks, organisations, and movements like the People's Health Movement, International Physicians for the Prevention of Nuclear War, Physicians for human rights, International council of Nurses, International Federation of Medical Students' Associations, Doctors without Borders, World Organization of Family Doctors, The Global Climate and Health Alliance, and Medicus Mundi International - Network health for All! 


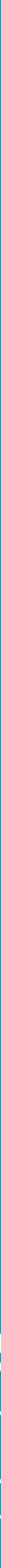


Abimbola, S. 2018. On the meaning of global health and the role of global health journals. Oxford: Oxford University Press.

Abimbola, S. 2019. The foreign gaze: Authorship in academic global health: BMJ Specialist Journals.

Abimbola, S. and S.M. Topp. 2018. Adaptation with robustness: The case for clarity on the use of 'resilience' in health systems and global health: BMJ Specialist Journals.

Ade, N., A. Rene, M. Khalifa, K.O. Babila, M.E. Monono, E. Tarcisse and J. NabyongaOrem. 2016. Coordination of the health policy dialogue process in Guinea: pre- and post-Ebola. BMC Health Serv Res 16 Suppl 4: 220.

Agence Nationale de Sécurité Sanitaire de Guinée. October 2016.

Aldis, W. 2008. Health security as a public health concept: A critical analysis. Health Policy and Planning 23, no 6: 369-75.

Aluttis, C., T. Bishaw and M.W. Frank. 2014. The workforce for health in a globalized context-global shortages and international migration. Global Health Action 7, no 1: 23611.

Amref. 2014. Position statement on community health workers. http://amref.org/ amref/en/info-hub/amref-position-statement-on $\neg$ community-healthworkers- $/$.

Arya, N. 2017. Peace and health: Bridging the north-south divide. Medicine, Conflict and Survival 33, no 2: 87-91.

Ashour, M. 2016. Applying resilience to health system research: Beyond a personal journey in the Gaza Strip. International Health Policies. 12 November 2016. https://www.internationalhealthpolicies.org/applying-resilience-to-healthsystem-research-beyond-a-personal-journey-in-the-gaza-strip/.

Assefa, Y., D. Tesfaye, W. Van Damme and P.S. Hill. 2018. Effectiveness and sustainability of a diagonal investment approach to strengthen the primary health-care system in Ethiopia. The Lancet 392, no 10156: 1473-81.

Atkinson, A.B. 2015a. Inequality - what can be done? Working paper 2. LSE International Inequalities Institute. November 2015. http://www.lse.ac.uk/ International-Inequalities/Assets/Documents/Working-Papers/WorkingPaper-2-Tony-Atkinson.pdf.

Atkinson, A.B. 2015b. Inequality: What can be done? Cambridge MA: Harvard University Press.

Bacchi, C. 2012. Introducing the 'What's the problem represented to be?' approach. Engaging with Carol Bacchi: Strategic interventions and exchanges: 21-24.

Bach, S. 2003. International migration of health workers: labour and social issues. Sectoral activities programme. Working paper WP.209. Geneva: International Labour Office. http://citeseerx.ist.psu.edu/viewdoc/ download?doi=10.1.1.90.4214\&rep=repl\&type=pdf.

Bailey, A.J. 2001. Worlds in motion: Understanding international migration at the 
end of the millennium: JSTOR.

Bakker, I.C. and S. Gill. 2011. Towards a new common sense: The need for new paradigms of global health. In Global health and global health ethics, eds Benatar, SR and Brock, G, UK, Cambridge: Cambridge University Press.329-32

Balasegaram, M., C. Brechot, J. Farrar, D. Heymann, N. Ganguly, M. Khor, Y. Levy, P. Matsoso, R. Minghui, B. Pecoul, L. Peilong, M. Tanner and J.A. Rottingen. 2015. A global biomedical R\&D fund and mechanism for innovations of public health importance. PLoS Med 12, no 5: el001831.

Balch, O. 2013. Buen vivir: the social philosophy inspiring movements in South America. The Guardian, 4 February 2013.

Banteyerga, H. 2011. Ethiopia's health extension program: improving health through community involvement. MEDICG Rev 13, no 3: 46-9.

Barder, O. 2014. The governance deficit. Owen Abroad (blog). http://www.owen. org/blog/7461.

Barnett, M. 2011. Empire of humanity: A history of humanitarianism. New York: Cornell University Press.

Barroy, H., J. Kutzin, A. Tandon, C. Kurowski, G. Lie, M. Borowitz, S. Sparkes and E. Dale. 2018. Assessing Fiscal Space for Health in the SDG Era: A Different Story. Health Systems \& Reform 4, no 1: 4-7.

Basic Income Earth Network. 2016. http://basicincome.org/.

Baudot, J. 2001. Building a World Community: Globalisation and the Common Good.

Copenhagen, Royal Danish Foreign Ministry for Foreign Affairs: Washington University Press.

Bauman, Z. 1998. Globalization: The human consequences. New York: Columbia University Press.

Beck, U. 2006a. The Cosmopolitan Vision. Cambridge: Polity Press.

Beck, U. 2006b. Living in the world risk society: A Hobhouse Memorial Public Lecture given on Wednesday 15 February 2006 at the London School of Economics. Economy and society 35, no 3: 329-45.

Beck, U. 2007. The cosmopolitan condition: Why methodological nationalism fails. Theory, culture \& society 24, no 7-8: 286-90.

Beck, U. 2010. The cosmopolitan manifesto. The cosmopolitanism reader: 217-28.

Beck, U. 2011. Cosmopolitanism as imagined communities of global risk. American behavioral scientist 55, no 10: 1346-61.

Beck, U. 2012. Global risk society. The Wiley-Blackwell Encyclopedia of Globalization.

Bemelmans, M. and M. Philips. 2017. New plan to tackle the global shortage of health workers fails to address economic constraints. The BMJ Opinion. 24 July 2017. https://blogs.bmj.com/bmj/2017/07/24/new-action-plan-toaddress-the-global-shortage-of-health-workers-fails-to-address-economicconstraints-to-its-implementation. 
Benatar, S.R. 1998. Global disparities in health and human rights: a critical commentary. Am J Public Health 88, no 2: 295-300.

Benatar, S.R. 2005. Moral imagination: the missing component in global health. PLoS Med 2, no 12: e400.

Benatar, S.R. 2015. Explaining and responding to the Ebola epidemic. Philos Ethics Humanit Med 10: 5.

Benatar, S.R. 2016. Politics, Power, Poverty and Global Health: Systems and Frames. Int J Health Policy Manag 5, no 10: 599-604.

Benatar, S.R., A.S. Daar and P.A. Singer. 2003. Global health ethics: the rationale for mutual caring. International Affairs 79, no 1: 107-38.

Benton, A. 2017. Whose security? Militarization and securitization during West Africa's Ebola outbreak. The Politics of Fear: Médecins Sans Frontières and the West African Ebola Epidemic: 25-50.

Berland, A., A. Best, K. Noel, J. Saul, J. Bitz, B. Barker and A. Wright. 2016. Assessing the legacy of the Global Health Workforce Alliance through the lens of Complex Adaptive Systems. Draft. InSource Research Group, 2016.

Best, A., A. Berland, T. Greenhalgh, I.L. Bourgeault, J.E. Saul and B. Barker. 2018. Networks as systems: A case study of the World Health Organisation's Global Health Workforce Alliance. Journal of health organization and management 32, no 1: 9-24.

Birn, A.-E., Y. Pillay and T.H. Holtz. 2017. Textbook of global health: Oxford University Press.

Blanchet, K., S.L. Nam, B. Ramalingam and F. Pozo-Martin. 2017. Governance and Capacity to Manage Resilience of Health Systems: Towards a New Conceptual Framework. Int J Health Policy Manag 6, no 8: 431-35.

Boothman, D. 2017. The sources for Gramsci's concept of hegemony. In Rethinking Gramsci, ed. Green, M, New York: Routledge.55-67

Bourgeault, I.L., R. Labonté, C. Packer, V. Runnels and G.T. Murphy. 2016. Knowledge and potential impact of the WHO Global code of practice on the international recruitment of health personnel: Does it matter for source and destination country stakeholders? Human Resources for Health 14, no 1: 25.

Bregman, R. 2017. Utopia for realists: And how we can get there: Bloomsbury Publishing.

Briody, C., L. Rubenstein, L. Roberts, E. Penney, W. Keenan and J. Horbar. 2018. Review of attacks on health care facilities in six conflicts of the past three decades. Conflict and health 12, no 1: 19.

Britnell, M. 2019. Human: Solving the global workforce crisis in healthcare: Oxford University Press.

Brolan, C.E. and P.S. Hill. 2016. Universal Health Coverage's evolving location in the post-2015 development agenda: Key informant perspectives within multilateral and related agencies during the first phase of post-2015 negotiations. Health Policy and Planning 31, no 4: 514-26. 
Brolan, C.E., P.S. Hill and G. Ooms. 2015. "Everywhere but not specifically somewhere": a qualitative study on why the right to health is not explicit in the post-2015 negotiations. BMC Int Health Hum Rights 15: 22-22.

Brown, G.W. and D. Held. 2010. The cosmopolitanism reader. Cambridge, UK: Polity.

Brown, G.W. and R. Labonté. 2011. Globalization and its methodological discontents: Contextualizing globalization through the study of HIV/AIDS. Globalization and health 7, no 1: 29.

Brown, T.M., M. Cueto and E. Fee. 2006. The World Health Organization and the transition from "international" to "global" public health. Am J Public Health 96, no 1: 62-72.

Brunswijck, G. 2018. Unhealthy conditions. IMF loan conditionality and its impact on health financing. https://eurodad.org/unhealthy-conditions.

Buchan, J., I.D. Couper, V. Tangcharoensathien, K. Thepannya, W. Jaskiewicz, G. Perfilieva and C. Dolea. 2013. Early implementation of WHO recommendations for the retention of health workers in remote and rural areas. Bulletin of the World Health Organization 91, no 11: 834-40.

Buchan, J., I.S. Dhillon and J. Campbell. 2017. Health employment and economic growth: an evidence base: World Health Organization.

Buchan, J., B. McPake, K. Mensah and G. Rae. 2009. Does a code make a difference - assessing the English code of practice on international recruitment. Human Resources for Health 7, no 1: 33.

Buchan, J., M. Wismar, I.A. Glinos and J. Bremner. 2014. Health professional mobility in a changing Europe. Copenhagen: World Health Organization.

Buhmann, G., J. Santa Barbara, N. Arya and K. Melf. 2010. The roles of the health sector and health workers before, during and after violent conflict. Medicine, Conflict and Survival 26, no 1: 4-23.

Busan Partnership Agreement. 2011. Fourth High Level Forum on Aid Effectiveness. OECD, Busan, South-Korea. http://www.oecd.org/dac/ effectiveness/49650173.pdf.

Buse, K. and S. Hawkes. 2015. Health in the sustainable development goals: ready for a paradigm shift? Global Health 11: 13.

Buse, K. and S. Hawkes. 2016. Sitting on the FENSA: WHO engagement with industry. The Lancet 388, no 10043: 446-7.

Buse, K., N. Mays and G. Walt. 2012. Making health policy: McGraw-Hill Education (UK). p.8-18

Bustreo, F. 2015. Financing the health Sustainable Development Goal. http://www. who.int/life-course/news/commentaries/financing-health-sustainable-goal/ en/.

Butler, D. 2011. Revamp for WHO. Nature 473, no 7348: 430-1.

Buzan, B., O. Wæver, O. Wæver and J. De Wilde. 1998. Security: A new framework for analysis: Lynne Rienner Publishers. 
Campbell, J. 2018. International Platform on Health Workforce Mobility:

An introduction https://www.who.int/hrh/migration/Introduction IntPlatMeetingl2Sept2018.pdf?ua=1 (accessed 18 December 2019).

Campbell, J., J. Buchan, G. Cometto, B. David, G. Dussault, H. Fogstad, I. Fronteira,

R. Lozano, F. Nyonator, A. Pablos-Mendez, E.E. Quain, A. Starrs and V.

Tangcharoensathien. 2013. Human resources for health and universal health coverage: fostering equity and effective coverage. Bulletin of the World Health Organization 91, no 11: 853-63.

Center for Public Health and Human Rights. 2013. Protection of Health Workers, Patients and Facilities in Times of Violence Center for Public Health and Human Rights, Johns Hopkins Bloomberg School of Public Health.

Chan Boegli, L. and M.G. Arcadu. 2017. Healing under fire-medical peace work in the field. Medicine, Conflict and Survival 33, no 2: 131-40.

Chandler, D. 2001. The road to military humanitarianism: How the human rights NGOs shaped a new humanitarian agenda. Human Rights Quarterly 23: 678.

Chandler, D. 2014. Beyond neoliberalism: resilience, the new art of governing complexity. Resilience 2, no 1: 47-63.

Chang, H.-J. 2005. Policy space in historical perspective. Paper presented at the Queen Elizabeth House 50th Anniversary Conference: The Development Threats and Promises, University of Oxford. 4-5 July 2005.

Chow, J.C. 2010. Is the WHO becoming irrelevant? Foreign Policy. http://www. foreignpolicy.com/articles/2010/12/08/is_the_who_becoming_irrelevant.

Chu, K.M., S. Jayaraman, P. Kyamanywa and G. Ntakiyiruta. 2014. Building research capacity in Africa: equity and global health collaborations. PLoS Med 11, no 3: el001612.

Clemens, M.A. 2011. The financial consequences of high-skill emigration: lessons from African doctors abroad. In Diaspora for development in Africa, eds Plaza, S and Ratha, D, Washington, D.C.: World Bank.p.165-82

Clemens, M.A. 2015. Global Skill Partnerships: a proposal for technical training in a mobile world. IZA Journal of Labor Policy 4, no 1: 2.

Clift, C. 2013. The Role of the World Health Organization in the International System. Centre on Global Health Security Working Group Papers. Chatham House. https://www.chathamhouse.org/sites/default/files/publications/ research/2013-02-01-role-world-health-organization-international-systemclift.pdf.

Cohen, D.A. 2019. Stop Eco-Apartheid: The Left's Challenge in Bolsonaro's Brazil. Dissent 66, no 1: 23-31.

Collinson, S. and S. Elhawary. 2012. Humanitarian space: a review of trends and issues. London: Humanitarian Policy Group, Overseas Development Institute Cometto, G., T. Boerma, J. Campbell, L. Dare and T. Evans. 2013. The Third Global Forum: framing the health workforce agenda for universal health coverage. 
The Lancet Global Health 1, no 6: e324-5.

Cometto, G., J. Buchan and G. Dussault. 2019. Developing the health workforce for universal health coverage. Bulletin of the World Health Organization.

Cometto, G., G. Ooms, A. Starrs and P. Zeitz. 2009. A global fund for the health MDGs? The Lancet 373, no 9674: 1500-2.

Cometto, G., R.M. Scheffler, T. Bruckner, J. Liu, A. Maeda, G. Tomblin-Murphy, D. Hunter and J. Campbell. 2017. Health workforce needs, demand and shortages to 2030. p 3-26. In Health Employment and Economic Growth: An Evidence Base, eds Buchan, J, Dhillon, IS and Campbell, J. Geneva: World Health Organization. https://www.who.int/hrh/resources/WHO-HLCReport_web.pdf?ua=1.

Crisis Group. 2019. Humanitarian Fallout of Conflict. https://www.crisisgroup.org/ humanitarian-fallout-conflict.

Crisp, N. 2010. Turning the world upside down: the search for global health in the 21st century: GRC Press.

Dal Poz, M.R., H.R. Sepulveda, M.H. Costa Couto, C. Godue, M. Padilla, R. Cameron and A. Vidaurre Franco Tde. 2015. Assessment of human resources for health programme implementation in 15 Latin American and Caribbean countries. Human Resources for Health 13: 24.

Dambisya, Y.M., N. Malema, C. Dulo, S. Matinhure and P. Kadama. 2013. The engagement of east and southern African countries on the WHO Code of Practice on the International Recruitment of Health Personnel and its implementation: EQUINET Discussion Paper 103. Harare: EQUINET.

Dambisya, Y.M., N. Malema, C. Dulo, S. Matinhure and P. Kadama. 2014. The engagement of east and southern African countries on the WHO Code of Practice on the International Recruitment of Health Personnel and its implementation. EQUINET Discussion Paper 103. June 2014 http://www. equinetafrica.org/sites/default/files/uploads/documents/GHD_Code_Diss_ Paper103.pdf.

Davies, S.E., A. Kamradt-Scott and S. Rushton. 2015. Disease diplomacy: International norms and global health security. Baltimore: JHU Press.

De Swaan, A. 1998. Project for a beneficial epidemic: On the collective aspects of contagion and prevention. Problems and Potential in International Health: Transdisciplinary Perspectives, Amsterdam, Het Spinhuis: 35-50.

De Waal, A. 2014. Militarizing global health. Boston Review 11.

De Walle, H. 2019. De Donut Economie https://vimeo.com/359548629.

Deacon, B. 2003. Global social governance reform. From institutions and policies to networks, projects and partnerships. Chapter 1. In Global social governance. Themes and prospects. Globalism and social policy programme 2003, eds Deacon, B, Ollila, E, Koivusalo, M and Stubbs, P. 
DeCoster, K. 2013. Is WHO ready for a rising Africa? http://archief. internationalhealthpolicies.org/who-ready-rising-africa/.

Department for International Development - UKaid. 2011. Assessment of the WHO. Multilateral aid review ensuring maximum value for money for UK aid through multilateral organisations. Annex 6. Multilateral aid review assessment summaries:206. https://assets.publishing.service.gov. uk/government/uploads/system/uploads/attachment_data/file/67583/ multilateral_aid_review.pdf.

Diallo, A. 2017. Minister of Health. Guinea. Plenary 6: The investor's forum: Fiscal policy and financing for jobs, health and inclusive growth. Fourth Global Forum on Human Resources for Health, Dublin, November 2017.

Diamond, L. 2015. Facing up to the democratic recession. Journal of Democracy 26, no 1: 141-55.

Dieleman, J.L., K. Cowling, I.A. Agyepong, S. Alkenbrack, T.J. Bollyky, J.B. Bump, C.S. Chen, K.A. Grépin, A. Haakenstad and A.C. Harle. 2019. The G20 and development assistance for health: historical trends and crucial questions to inform a new era. The Lancet.

Dieleman, M., S. Kane, P. Zwanikken, B. Gerretsen and W.H. Organization. 2011. Realist review and synthesis of retention studies for health workers in rural and remote areas.

Drezner, D.W. 2013. The rise of "good enough" global governance? https:// foreignpolicy.com/2013/01/08/the-rise-of-good-enough-globalgovernance/.

Dussault, G. 2019. Reflections on Health Workforce Development: Comment on” Health Professional Training and Capacity Strengthening Through International Academic Partnerships: The First Five Years of the Human Resources for Health Program in Rwanda”. International journal of health policy and management 8, no 4: 245.

Dussault, G., E. Badr, H. Haroen, M. Mapunda, A.S.T. Mars, K. Pritasari and G. Cometto. 2016. Follow-up on commitments at the Third Global Forum on Human Resources for Health: Indonesia, Sudan, Tanzania. Human Resources for Health 14, no 1: 16.

Dussault, G. and J. Buchan. 2014. The economic crisis in the EU: impact on health workforce mobility - Chapter 3 In Health professional mobility in a changing Europe - New dynamics, mobile individuals and diverse responses, eds Buchan, J, Wismar, M, Glinos, IA and Bremner, J, London: European Observatory on Health Systems and Policies.p. 35-64

Dybul, M., P. Piot and J. Frenk. 2012. Reshaping global health. Policy Review 173: 3-18. Earth Charter Initiative. 2015. http://earthcharter.org/.

ECORYS Consortium de Santé. 2016. Audit Institutionnel, Organisationnel et Fonctionnel du Ministère de la Santé - République de Guinée. European 
Commission Directorate for Development Cooperation.

El-Erian, M. 2019. Argentina's economic crisis is the result of avoidable mistakes.

The Guardian 10 September 2019.

Elbe, S. 2012. Bodies as battlefields: toward the medicalization of insecurity.

International Political Sociology 6, no 3: 320-22.

Elliott, L. 2019. IMF accused of 'reckless lending' to debt-troubled states The Guardian 7 October 2019.

EU Joint Action Health Workforce Planning and Forecasting. 2015. The applicability of the WHO Global Code of Practice on the International Recruitment of Health Personnel within a European context. WP4. Hungary: Semmelweis University, Health Services Management Training Centre. http:// healthworkforce.eu/wp-content/uploads/2015/10/150609_wp4_who_ applicabilty_report.pdf.

European Commission. 2010. The EU Role in Global Health. Brussels: European Commission.

European Commission. 2012a. Communication towards a job rich recovery. European Commission: Strasbourg.

European Commission. 2012b. Staff working document on an action plan for the EU Health Workforce. Strasbourg: European Commission.

European Observatory on Health Systems and Policies. 2016. Health system reviews (HiT series). http://www.euro.who.int/en/about-us/partners/observatory/ publications/health-system-reviews-hits.

Evans, B. and J. Reid. 2015. Exhausted by resilience: response to the commentaries. Resilience 3, no 2: 154-59.

Evans, T., E.C. Araujo, C.H. Herbst and O. Pannenborg. 2016. Addressing the challenges of health professional education: opportunities to accelerate progress towards universal health coverage. In World Innovation Summit for Health, 2016. Doha, Qatar.

Extinction Rebellion. 2019. Doctors for Extinction Rebellion. https://www. doctorsforxr.com/.

Fahy, N., T. Hervey, S. Greer, H. Jarman, D. Stuckler, M. Galsworthy and M. McKee. 2019. How will Brexit affect health services in the UK? An updated evaluation. The Lancet 393, no 10174: 949-58.

Faki Mahamat, M. 2017. Speech of the chairperson of the commission of the African Union. 5th African Union- European Union summit. Abidjan, Cote D'Ivoire: 2017. https://au.int/sites/default/files/speeches/33424-sp-speechnof_moussa_ faki.pdf.

Farmer, P. 2014. Diary. London Review of Books 36, no 20: 38-39.

Fidler, D.P. 2001. The globalization of public health: the first 100 years of international health diplomacy. Bulletin of the World Health Organization 79, no 9: 842-9. 
Fidler, D.P. 2003. SARS: political pathology of the first post-Westphalian pathogen. The Journal of Law, Medicine \& Ethics 31, no 4: 485-505.

Fidler, D.P. 2007. Architecture amidst anarchy: Global health's quest for governance. Global Health Governance 1, no 1: 1-17.

Fidler, D.P. 2009. After the Revolution: Global Health Politics in a Time of Economic Crisis and Threatening Future Trends”. Articles by Maurer Faculty. Paper 145. http://www.repository.law.indiana.edu/facpub/145.

Fidler, D.P. 2010. The Challenges of Global Health Governance. Working Paper. Council on Foreign Relations, New York, p.3. https://www.cfr.org/sites/ default/files/pdf/2010/05/IIGG_WorkingPaper4_GlobalHealth.pdf.

Fieno, J.V., Y.M. Dambisya, G. George and K. Benson. 2016. A political economy analysis of human resources for health (HRH) in Africa. Human Resources for Health 14, no 1: 44.

Food and Agriculture Organization of the United Nations Council. 2013. FAO Strategy for Partnerships with Civil Society Organizations. 146th session. http://www.fao.org/3/MF999E/MF999E.pdf.

Foro Nacional de Salud de El Salvador. https://fnssv.com/.

Fouad, F.M., A. Sparrow, A. Tarakji, M. Alameddine, F. El-Jardali, A.P. Coutts, N. El Arnaout, L.B. Karroum, M. Jawad and S. Roborgh. 2017. Health workers and the weaponisation of health care in Syria: a preliminary inquiry for The Lancet-American University of Beirut Commission on Syria. The Lancet 390, no 10111: 2516-26.

Foucault, M. 2002. The birth of the clinic: Routledge.

Foucault, M. 2009. Security, territory, population: lectures at the Colle ge de France, 1977-78. London: Palgrave Macmillan.

Foucault, M., A.I. Davidson and G. Burchell. 2008. The birth of biopolitics: lectures at the Collège de France, 1978-1979: Springer.

Fraser, N. 2016. Contradictions of capital and care. New Left Review 100, no 99: 117.

Frenk, J., L. Chen, Z.A. Bhutta, J. Cohen, N. Crisp, T. Evans, H. Fineberg, P. Garcia, Y. Ke, P. Kelley, B. Kistnasamy, A. Meleis, D. Naylor, A. Pablos-Mendez, S. Reddy, S. Scrimshaw, J. Sepulveda, D. Serwadda and H. Zurayk. 2010. Health professionals for a new century: transforming education to strengthen health systems in an interdependent world. The Lancet 376, no 9756: 1923-58.

Frenk, J. and S. Moon. 2013. Governance challenges in global health. New England Journal of Medicine 368, no 10: 936-42.

Fried, L.P., M.E. Bentley, P. Buekens, D.S. Burke, J. Frenk, M.J. Klag and H.C. Spencer. 2010. Global health is public health. The Lancet 375, no 9714: 535-7.

Friedman, T.L. 2000. The Lexus and the olive tree: Understanding globalization. New York: Farrar, Straus and Giroux.

Fukuyama, F. 1989. The end of history? The National Interest. https://www. wesjones.com/eoh.htm. 
Fuller Torrey, E. 2014. How the U.S. made the Ebola crisis worse. The Wall Street Journal. https:/www.wsj.com/articles/e-fuller-torrey-how-the-u-s-madethe-ebola-crisis-worse-1413328767.

G8 Communique. 2010. Annex I: the G8 muskoka initiative: maternal, newborn and under-five child health: The Partnership for Maternal, Newborn \& Child health and World Health Organization, 2010. http:// www.who.int/pmnch/ media/g8watch_2010/en/.

Gagnon, M.L. and R. Labonté. 2013. Understanding how and why health is integrated into foreign policy - a case study of health is global, a UK Government Strategy 2008-2013. Global Health 9, no 1: 24.

Galeano, E.H. 1993. Las Palabres Andantes. Argentina: Catálogos SRL. https://static. telesurtv.net/filesOnRFS/news/2015/04/13/laspalabrasandantes.pdf.

Gebauer, T. 2012. High time to re-politicise NGOs, aiding at change or abetting crimes. In: Paper prepared for the Third People's Health Assembly (PHA3) e Cape Town. https://www.medico.de/en/aiding-change-or-abettingcrimes-16812/.

George, A., K. Scott, V. Govender and W.H. Organization. 2017. A health policy and systems research reader on human resources for health: World Health Organization.

Ghebreyesus, T.A. 2018. Towards Universal Health Coverage: Tackling the Health Financing Crisis to End Poverty. Washington: The World Bank.

Ghodsee, K.R. 2019. Gendered impacts of privatisation and austerity in eastern Europe. The Lancet 393, no 10171: 519-20.

Gill, S. 2015. Reimagining the Future: Some Critical Reflections. In Critical Perspectives on the Crisis of Global Governance: Reimagining the Future, ed. Gill, S, 1-23: Springer; https://link.springer.com/chapter/10.1057/9781137441409_1.

Gill, S. and S.R. Benatar. 2016a. Global Health Governance and Global Power: A Critical Commentary on the Lancet-University of Oslo Commission Report. Int J Health Serv 46, no 2: 346-65.

Gill, S. and S.R. Benatar. 2016b. History, Structure and Agency in Global Health Governance Comment on "Global Health Governance Challenges 2016 - Are We Ready?”. Int J Health Policy Manag 6, no 4: 237-41.

Giraldi, P. 2018. More Lies About Syria's “White Helmets”. In Global Research: Centre for Research on Globalization

Global Burden of Disease Study 2013 Collaborators. 2015. Global, regional, and national incidence, prevalence, and years lived with disability for 301 acute and chronic diseases and injuries in 188 countries, 1990-2013: a systematic analysis for the Global Burden of Disease Study 2013. The Lancet 386, no 9995: 743-800.

Global Health Security. 2019. Sydney Statement on Global Health Security. In Global Health security 2019. 
Global Health Watch. People's Health Movement. WHO-watch. Democratising global health governance. . http://www.ghwatch.org/democratising.

Global Health Watch. 2014. An Alternative World Health Report. The Global Health Workforce Crisis. London: Zed Books. p. 158

Global Health Workforce Alliance. 2011. Imagine...A health worker for everyone, everywhere

Global Health Workforce Alliance/WHO. 2012. Country Coordination and Facilitation (CCF)-resources. http://www.who.int/workforcealliance/ knowledge/resources/ccfresources/en/

Global Health Workforce Alliance/WHO. 2013a. Country coordination and facilitation: South Sudan. http://www.who.int/workforcealliance/countries/ ssd/en/

Global Health Workforce Alliance/WHO. 2013b. A Universal Truth: No Health Without a Workforce. Third Global Forum on Human Resources for Health Report. https:/www.who.int/workforcealliance/knowledge/resources/ GHWA-a_universal_truth_report.pdf?ua=1.

Global Health Workforce Alliance/WHO. 2014. Analysis of the commitments made by national governments of 57 countries at the Third Global Forum on human resources for health. www.who.int/workforcealliance/knowledge/ resources/Analysis_HRH_commitments_13Mar2015_c_SR_LAST.pdf

Global Policy Watch. 2015. Civil Society Response to the Addis Ababa Action Agenda on Financing for Development .16 July 2015, Addis Ababa, Bonn, Germany. https://www.globalpolicywatch.org/blog/2015/07/16/civil-society-responseagenda-financingdevelopment/.

Global Polio Eradication Initiative. 2018. Global Polio Eradication Initiative: annual report 2017: World Health Organization

Gómez, E.J., F.A. Perez and D. Ventura. 2018. What explains the lacklustre response to Zika in Brazil? Exploring institutional, economic and health system context. BMJ global health 3, no 5: e000862.

Gostin, L.O. and E.A. Friedman. 2014. Ebola: a crisis in global health leadership. Lancet 384, no 9951: 1323-5.

Gostin, L.O. and E.A. Friedman. 2015. A retrospective and prospective analysis of the west African Ebola virus disease epidemic: robust national health systems at the foundation and an empowered WHO at the apex. The Lancet 385, no 9980: 1902-9.

Gostin, L.O., E.A. Friedman, K. Buse, A. Waris, M. Mulumba, M. Joel, L. Dare, A. Dhai and D. Sridhar. 2013. Towards a framework convention on global health. Bulletin of the World Health Organization 91, no 10: 790-3.

Gostin, L.O., D. Lucey and A. Phelan. 2014. The Ebola epidemic: a global health emergency. JAMA 312, no 11: 1095-6.

Gostin, L.O. and D. Sridhar. 2014. Global health and the law. New England Journal of 
Medicine 370, no 18: 1732-40.

Gostin, L.O., D. Sridhar and D. Hougendobler. 2015. The normative authority of the World Health Organization. public health 129, no 7: 854-63.

Greenhalgh, T. and C. Papoutsi. 2018. Studying complexity in health services research: desperately seeking an overdue paradigm shift: BioMed Central.

Guild, E. 2019. The UN Global Compact for Safe, Orderly and Regular Migration: What Place for Human Rights? International Journal of Refugee Law 30, no 4: 661-63.

Guterres, A. 2019. Report of the Secretary-General on SDG Progress 2019: United Nations

Habermas, J. 1996. Between Facts and Norms. Contributions to a Discourse Theory of Law and Democracy. Cambridge: Polity Press.

Habermas, J. 2018. The postnational constellation: Political essays: John Wiley \& Sons.

Hale, T., D. Held and K. Young. 2013a. Gridlock: From Self-reinforcing Interdependence to Second-order Cooperation Problems. Global Policy 4, no 3: 223-35.

Hale, T., D. Held and K. Young. 2013b. Gridlock: why global cooperation is failing when we need it most. Cambridge, UK: Polity.

Hammonds, R., G. Ooms, M. Mulumba and A. Maleche. 2019. UHC2030's Contributions to Global Health Governance that Advance the Right to Health Care: A Preliminary Assessment.

Hammonds, R., G. Ooms and W. Vandenhole. 2012. Under the (legal) radar screen: global health initiatives and international human rights obligations. BMC Int Health Hum Rights 12: 31.

Harman, S. 2016. The Bill and Melinda Gates Foundation and Legitimacy in Global Health Governance. Global Governance 22: 349-68.

Harman, S. and C. Wenham. 2018. Governing Ebola: between global health and medical humanitarianism. Globalizations 15, no 3: 362-76.

Harman, S. and D. Williams. 2013. Governing the world? Cases in global governance. UK: Routledge.

Harmer, A. 2012. Who's Funding WHO? http://andrewharmer.org/wp-content/ uploads/2017/06/Who\%E2\%80\%99s-funding-WHO-\%E2\%80\%93globalhealthpolicy.net_.pdf.

Harmer, A. and K. Buse. 2014. The BRICS - a paradigm shift in global health? Contemporary Politics 20, no 2: 127-45.

Harmer, A., Y. Xiao, E. Missoni and F. Tediosi. 2013. 'BRICS without straw’? A systematic literature review of newly emerging economies' influence in global health. Global Health 9, no 1: 15.

Hawkes, N. and S. Arie. 2014. Is the United Nations catching up with Ebola at last? bmj 349: g6576. 
Health Systems Research. 2016. Vancouver Statement for the Fourth Global Symposium on Health Systems Research. Vancouver, Canada. http:// healthsystemsresearch.org/hsr2016/wp-content/uploads/VancouverStatement-FINAL.pdf.

Health Workforce Advocacy Initiative. 2009. Incorporating the right to Health into Health Workforce Plans: Key considerations. Physicians for Human Rights. https://s3.amazonaws.com/PHR_other/incorporating-right-to-health.pdf. HealthWorkers4All. HealthWorkers4All - Amref. Case Study. Italy: The Contact Point for migrant health workers organised by IPASVI Florence. http://www. healthworkers4all.eu/fileadmin/docs/eu/hw4all_papers/Case_StudiesITAIPASVI_.pdf.

HealthWorkers4All. HealthWorkers4All - Amref. Case Study. Italy: The FNOMCeO commission on development cooperation. http://www.healthworkers4all. eu/fileadmin/docs/eu/hw4all_papers/Case_StudieIT-FNOMCeO_.pdf.

HealthWorkers4All. HealthWorkers4All - Centrul pentru Politici și Servicii de Sănătate. Case Study. Romania: Cross-border cooperation covering the need for human resources in Calarasi County Emergency Hospital - employing specialist MDs from Bulgaria. http://www.healthworkers4all.eu/fileadmin/ docs/eu/hw4all_papers/Best_Practice_from_Romania_1.pdf.

HealthWorkers4All. HealthWorkers4All - Health Poverty Action. Case Study. UK: Royal College of Nursing (RCN) Labour Market Review. http://www. healthworkers4all.eu/fileadmin/docs/eu/hw4all_papers/Case_StudiesUKrcn.pdf.

HealthWorkers4All. HealthWorkers4All - Medicus Mundi. Case Study. Spain: The migration experience of Spanish nurses in Germany. http:// www.healthworkers4all.eu/fileadmin/docs/eu/hw4all_papers/Case_ StudiesSPANJE-HC.pdf.

HealthWorkers4All. HealthWorkers4All - Memisa. Case Study. Belgium: Charter proposed by Belgian development cooperation actors on the recruitment and support for the development of human resources for health (HRH) in partner countries - November 2012. http://www.healthworkers4all.eu/ fileadmin/docs/eu/hw4all_papers/Case_StudiesBelgie_charter.pdf.

HealthWorkers4All. HealthWorkers4All - Memisa. Case Study. Belgium: Memisa 'HOSPITAL FOR HOSPITAL' twinning programme. http://www. healthworkers4all.eu/fileadmin/docs/eu/hw4all_papers/Case_StudiesBelgieH4H.pdf.

HealthWorkers4All. HealthWorkers4All - Redemptoris Missio. Case Study. Poland: Attempt to obtain reliable data on the scale of migration of Polish medical personnel. http://www.healthworkers4all.eu/fileadmin/docs/eu/hw4all_ papers/Case_StudiesPolenAttempt_.pdf.

HealthWorkers4All. HealthWorkers4All - Terre des Hommes. Gase Study. Germany: 
The Germany-Philippines bilateral agreement about the recruitment of nurses. http://www.healthworkers4all.eu/fileadmin/docs/eu/hw4all_papers/ Best_Practice_Examples_from_Germany.pdf.

HealthWorkers4All. HealthWorkers4All - Terre des Hommes. Case Study. Germany: The struggle for decent work in nursing at the Berlin hospital Charité. http:// www.healthworkers4all.eu/fileadmin/docs/eu/hw4all_papers/Best_Practice_ Examples_from_Germany.pdf.

HealthWorkers4All. HealthWorkers4All - Wemos. Case Study. The Netherlands:

Caring for carers in Dutch home-based care. http://www.healthworkers4all. eu/fileadmin/docs/eu/hw4all_papers/Case_StudiesNLcaring.pdf.

HealthWorkers4All. HealthWorkers4All - Wemos. Case Study. The Netherlands: Corporate Social Responsibility in favour of the global health workforce. http://www.healthworkers4all.eu/fileadmin/docs/eu/hw4all_papers/Case_ StudiesNLcorp.pdf.

HealthWorkers4All. Online Collaboration Platform. European Call for Action. A Health Worker for Everyone, Everywhere! Call to Action for European Decision-makers, Towards Strong Health Workforces and Sustainable Health Systems Around the World. http://www.healthworkers4all.eu/eu/ contributions/european-call-to-action/.

Healthworkers4All. 2015. Collection of case studies. Practices of WHO Code implementation in Europe: the role of non-governmental actors.

Held, D. 2006. Reframing global governance: Apocalypse soon or reform! New Political Economy 11, no 2: 157-76.

Held, D., I. Kickbusch, K. McNally, D. Piselli and M. Told. 2019. Gridlock, innovation and resilience in global health governance. Global Policy 10, no 2: 161-77.

Heller, P.S. 2005. Understanding fiscal space. Washington D.C.: International Monetary Fund.

Hervey, G. 2017. The EU Exodus: When doctors and nurses follow the money. Politico. eu, Sept 27.

Hickel, J. 2015a. Five reasons to think twice about the UN's Sustainable Development Goals. LSE Blog. 23 September 2015. https://blogs.lse. ac.uk/southasia/2015/09/23/five-reasons-to-think-twice-about-the-unssustainable-development-goals/.

Hickel, J. 2015b. The Problem with Saving the World. The UN's new Sustainable Development Goals aim to save the world without transforming it. Jacobin Magazine. https:/www.jacobinmag.com/2015/08/global-poverty-climatechange-sdgs.

Hickel, J. 2017. The Divide: A Brief Guide to Global Inequality and its Solutions. London: William Heinemann.

Hickel, J. 2019. Degrowth: a theory of radical abundance. real-world economics review: 54 . 
Hickel, J. 2020. The sustainable development index: Measuring the ecological efficiency of human development in the anthropocene. Ecological Economics 167: 106331.

Hill, P.S. 2011. Understanding global health governance as a complex adaptive system. Global Public Health 6, no 6: 593-605.

Hill, P.S., P. Vermeiren, K. Miti, G. Ooms and W. Van Damme. 2011. The Health Systems Funding Platform: Is this where we thought we were going? Globalization and health 7, no 1: 16.

Hoffman, S.J. and J.-A. Røttingen. 2013. Dark sides of the proposed framework convention on Global Health's many virtues: a systematic review and critical analysis. health and human rights 15, no 1: 117-34.

Hoffman, S.J. and J.-A. Røttingen. 2014. Split WHO in two: strengthening political decision-making and securing independent scientific advice. public health 128, no 2: 188-94.

Honwana, A. 2014. 2 'Waithood': Youth Transitions and Social Change. In Development and Equity: Brill.28-40

Horton, R. 2014. Offline: 2015-the year to rebuild WHO. The Lancet 384, no 9952: 1412. Horton, R. 2019. Offline: WHO-the story so far... The Lancet 393, no 10187: 2184.

Horton, R., R. Beaglehole, R. Bonita, J. Raeburn, M. McKee and S. Wall. 2014. From public to planetary health: a manifesto. The Lancet 383, no 9920: 847.

Human Resources for Health Country Commitments. 2015. Case studies from the Dominican Republic, Kenya, Mali and Uganda: IntraHealth International. http://www.intrahealth.org/files/media/ hrhcountrycommitmentscasestudies/HRH_Commitments_Case_Studies.pdf

ICRC. 2017. Health Care in Danger http://healthcareindanger.org/the-issue/.

INDEPTH Network. 2016. Better Health Information for Better Health Policy. http:// www.indepth-network.org/

Institute for Health Metrics and Evaluation. 2011. Financing global health in 2011: continued growth as MDG deadline approaches. Seattle, WA: IHME. . http:// www.healthmetricsandevaluation.org/sites/default/.les/policy_report/2011/ FGH_2011_overview_IHME.pdf.

Institute of Development Studies. 2013. Making the most of resilience. IDS in Focus Policy Briefing. Issue 32. https://www.ids.ac.uk/publications/making-themost-of-resilience/.

International Commission of Jurists. 2012. Maastricht Principles on Extraterritorial Obligations of States in the area of Economic, Social and Cultural Rights, February 2012. https:/www.etoconsortium.org/nc/en/main-navigation/ library/maastricht-principles/?tx_drblob_pil\%5BdownloadUid\%5D=23.

International Labour Organisation. 2012. R202 - Social Protection Floors Recommendation, 2012 (No. 202). Recommendation concerning National Floors of Social Protection. Adoption: Geneva, 101st ILC session (14 June 
2012). https://www.ilo.org/dyn/normlex/en/f?p=NORMLEXPUB:12100:0::NO: :P12100_ILO_CODE:R202.

International Labour Organisation. 2019. Work for a Brighter Future. Global commission on the future of work

International Monetary Fund. 2015. IMF Country Report Malawi No 15/345. https:// www.imf.org/external/pubs/ft/scr/2015/cr15345.pdf.

International Monetary Fund. 2019a. Country information

International Monetary Fund. 2019b. A Strategy for IMF Engagement on Social Spending.

Jamison, D.T., L.H. Summers, G. Alleyne, K.J. Arrow, S. Berkley, A. Binagwaho, F. Bustreo, D. Evans, R.G.A. Feachem, J. Frenk, G. Ghosh, S.J. Goldie, Y. Guo, S. Gupta, R. Horton, M.E. Kruk, A. Mahmoud, L.K. Mohohlo, M. Ncube, A. Pablos-Mendez, K.S. Reddy, H. Saxenian, A. Soucat, K.H. Ulltveit-Moe and G. Yamey. 2013. Global health 2035: a world converging within a generation. The Lancet 382, no 9908: 1898-955.

Jansen, C., L. Codjia, G. Cometto, M.L. Yansane and M. Dieleman. 2014. Realizing universal health coverage for maternal health services in the Republic of Guinea: the use of workforce projections to design health labor market interventions. Risk Manag Healthc Policy 7: 219-32.

Jesus, T.S., E. Kondilis, J. Filippon and G. Russo. 2019. Impact of economic recessions on healthcare workers and their crises' responses: study protocol for a systematic review of the qualitative and quantitative evidence for the development of an evidence-based conceptual framework. BMJ open 9, no 11.

Joint Learning Initiative. 2004. Human resources for health: overcoming the crisis. Cambridge: Global Equity Initiative, Harvard University. https://www.who. int/hrh/documents/JLi_hrh_report.pdf.

Joseph, J. 2013. Resilience as embedded neoliberalism: a governmentality approach. Resilience 1, no 1: 38-52.

Jutz, R. 2015. The role of income inequality and social policies on income-related health inequalities in Europe. Int J Equity Health 14: 117.

Kamradt-Scott, A., S. Harman, C. Wenham and F. Smith. 2016. Civil-military cooperation in Ebola and beyond. The Lancet 387, no 10014: 104-05.

Kamradt-Scott, A., S. Harman, C. Wenham and F. Smith III. 2015. Saving lives: the civil-military response to the 2014 Ebola outbreak in west Africa. Sydney: The University of Sydney.

Kamradt-Scott, A. and M. Sangiorgio. 2013. Reforming the WHO: progress to date in restructuring global health's foremost institution. Health Diplomacy Monitor 4, no 11-3.

Karanikolos, M., P. Mladovsky, J. Cylus, S. Thomson, S. Basu, D. Stuckler, J.P. Mackenbach and M. McKee. 2013. Financial crisis, austerity, and health in Europe. The Lancet 381, no 9874: 1323-31. 
Karesh, W.B., A. Dobson, J.O. Lloyd-Smith, J. Lubroth, M.A. Dixon, M. Bennett, S. Aldrich, T. Harrington, P. Formenty, E.H. Loh, C.C. Machalaba, M.J. Thomas and D.L. Heymann. 2012. Ecology of zoonoses: natural and unnatural histories. The Lancet 380, no 9857: 1936-45.

Kauppi, M.V. and P.R. Viotti. 2009. International relations theory. Harlow: Pearson Longman.

Kennedy, J., M. McKee and L. King. 2015. Islamist insurgency and the war against polio: A cross-national analysis of the political determinants of polio. Globalization and health 11, no 1: 40.

Kentikelenis, A., L. King, M. McKee and D. Stuckler. 2015. The International Monetary Fund and the Ebola outbreak. The Lancet Global Health 3, no 2: e6970 .

Kentikelenis, A.E. 2017. Structural adjustment and health: A conceptual framework and evidence on pathways. Social Science \& Medicine 187: 296-305.

Keohane, R.O. 2006. The Contingent Legitimacy of Multilateralism. GARNET Working Paper: No: 09/06 https://warwick.ac.uk/fac/soc/pais/research/ researchcentres/csgr/garnet/workingpapers/0906.pdf.

Khan, F.A., S. Chikkatagaiah, M. Shafiullah, M. Nasiri, A. Saraf, T. Sehgal, A. Rana, G. Tadros and P. Kingston. 2015. International medical graduates (IMGs) in the UK-a systematic review of their acculturation and adaptation. Journal of international migration and integration 16, no 3: 743-59.

Kickbusch, I. 2006. Mapping the future of public health: action on global health. Can J Public Health 97, no 1: 6-8.

Kickbusch, I. 2011. Global health diplomacy: how foreign policy can influence health. bmj 342: d3154.

Kickbusch, I. 2013a. A Game Change in Global Health: The Best Is Yet to Come. Public Health Reviews 35, no 1: 2.

Kickbusch, I. 2013b. WHO reform: A personal perspective. Journal of Public Health Policy 34, no 3: 481-85.

Kickbusch, I. 2016. Global Health Governance Challenges 2016 - Are We Ready? Int J Health Policy Manag 5, no 6: 349-53.

Kickbusch, I., W. Hein and G. Silberschmidt. 2010. Addressing Global Health Governance Challenges through a New Mechanism: The Proposal for a Committee C of the World Health Assembly. The Journal of Law, Medicine \& Ethics 38, no 3: 550-63.

Kickbusch, I. and K. Reddy. 2015. Global health governance-the next political revolution. public health 129, no 7: 838-42.

Kickbusch, I. and M.M.C. Szabo. 2014. A new governance space for health. Glob Health Action 7: 23507.

Kieny, M.-P., B.D. Evans, G. Schmets and S. Kadandale. 2014. Health-system resilience: reflections on the Ebola crisis in western Africa. Bulletin of the 
World Health Organization 92, no 850.

Kieny, M.P., H. Bekedam, D. Dovlo, J. Fitzgerald, J. Habicht, G. Harrison, H. Kluge, V. Lin, N. Menabde, Z. Mirza, S. Siddiqi and P. Travis. 2017. Strengthening health systems for universal health coverage and sustainable development. Bulletin of the World Health Organization 95, no 7: 537-39.

Kieny, M.P. and D. Dovlo. 2015. Beyond Ebola: a new agenda for resilient health systems. The Lancet 385, no 9963: 91-92.

Kirton, J. and I. Kickbusch. 2019. Health is a Political Choice https://bit.ly/2019UHC.

Klein, N. 2016. Let Them Drown. The Violence of Othering in a Warming World. London Review of Books. 2016;38(11):11-14. http://www.lrb.co.uk/v38/nll/ naomi-klein/let-them-drown.

Klosse, S. and J. Muysken. 2016. Curbing the Labor Market Divide by fostering Inclusive Labor Markets through a Job Guarantee Scheme. Psychosociological Issues in Human Resource Management 4, no 2: 185-219.

Kluge, H., J.M. Martín-Moreno, N. Emiroglu, G. Rodier, E. Kelley, M. Vujnovic and G. Permanand. 2018. Strengthening global health security by embedding the International Health Regulations requirements into national health systems. BMJ global health 3, no Suppl 1: e000656.

Koenig-Archibugi, M. 2010. Is global democracy possible? Eur J Int Relat. Earlier version available at: http://personal.lse.ac.uk/koenigar/Koenig-Archibugi_Is_ Global_Democracy_Possible.pdf; 16 June 2010.

Kolb, S., P. Weindling, V. Roelcke and H. Seithe. 2012. Apologising for Nazi medicine: a constructive starting point. The Lancet 380, no 9843: 722-23.

Kolehmainen-Aitken, R.-L. 2004. Decentralization's impact on the health workforce: Perspectives of managers, workers and national leaders. Human Resources for Health 2, no 1: 5.

Kolie, D., A. Delamou, R. van de Pas, N. Dioubaté, P. Bouedouno, A.H. Beavogui, A. Kaba, A. Diallo, W. van De Put and W. van Damme. 2019. "Never let a crisis go to waste": policy actors, Ebola outbreak and agenda-setting for health system strengthening in Guinea. BMJ global health 4:e001925.

Kolie, D., R. van de Pas, D. Alexandre, W. Van Damme, S. Van Belle and A. Beavogui. 2017. Analyse situationnelle du personnel de santé et des établissements de formation pour le renforcement du système de santé Guinee. https:// www.researchgate.net/publication/323541291_Analyse_situationnelle_ du_personnel_de_sante_et_des_etablissements_de_formation_pour_le_ renforcement_du_systeme_de_sante_Guinee.

Koon, A.D., B. Hawkins and S.H. Mayhew. 2016. Framing and the health policy process: a scoping review. Health Policy and Planning 31, no 6: 801-16.

Koplan, J.P., T.C. Bond, M.H. Merson, K.S. Reddy, M.H. Rodriguez, N.K. Sewankambo and J.N. Wasserheit. 2009. Towards a common definition of global health. The Lancet 373, no 9679: 1993-95. 
Kruk, M.E., M. Myers, T.S. Varpilah and B.T. Dahn. 2015. What is a resilient health system? Lessons from Ebola. The Lancet 385: 1910-12.

Kurniati, A., E. Rosskam, M.M. Afzal, T.B. Suryowinoto and A.G. Mukti. 2015. Strengthening Indonesia's health workforce through partnerships. public health 129, no 9: 1138-49.

Kutzin, J. and S.P. Sparkes. 2016. Health systems strengthening, universal health coverage, health security and resilience. Bulletin of the World Health Organization 94, no 1: 2.

Kvangraven, I.H. 2015. Can development goals help development finance? If so, how? https://www.globalpolicywatch.org/blog/2015/10/20/developmentgoals-help-finance/.

Labonté, R. 2016. Health Promotion in an Age of Normative Equity and Rampant Inequality. Int J Health Policy Manag 5, no 12: 675-82.

Labonté, R., C. Blouin, M. Chopra, K. Lee, C. Packer, M. Rowson, T. Schrecker and D. Woodward. 2007. Towards health-equitable globalisation: rights, regulation and redistribution.

Labonté, R. and M.L. Gagnon. 2010. Framing health and foreign policy: lessons for global health diplomacy. Globalization and health 6, no 1: 14.

Labonté, R. and A. Ruckert. 2019. Health Equity in a Globalizing Era: Past Challenges, Future Prospects. Oxford: Oxford University Press.

Labonté, R. and T. Schrecker. 2009. Rights, Redistribution, and Regulation. Chapter 13. In Globalization and health: pathways, evidence and policy, eds Labonté, R, Schrecker, T, Packer, C and Runnels, V, London: Routledge.327-33

Langer, A., A. Meleis, F.M. Knaul, R. Atun, M. Aran, H. Arreola-Ornelas, Z.A. Bhutta, A. Binagwaho, R. Bonita, J.M. Caglia, M. Claeson, J. Davies, F.A. Donnay, J.M. Gausman, C. Glickman, A.D. Kearns, T. Kendall, R. Lozano, N. Seboni, G. Sen, S. Sindhu, M. Temin and J. Frenk. 2015. Women and Health: the key for sustainable development. The Lancet 386, no 9999: 1165-210.

Lauer, J., A. Soucat, E. Araujo, M. Bertram, T. Edejer, C. Brindley, E. Dale and A. Tan. 2017a. Paying for needed health workers for the SDGs:an analysis of fiscal and financial space. p 236. In Health Employment and Economic Growth: An Evidence Base, eds Buchan, J, Dhillon, IS and Campbell, J. Geneva: World Health Organization. https://www.who.int/hrh/resources/WHO-HLCReport_web.pdf?ua=1.

Lauer, J., A. Soucat, E. Araujo and D. Weakliam. 2017b. Pathways: the health system, health employment, and economic growth. Health. p 174. In Health Employment and Economic Growth: An Evidence Base, eds Buchan, J, Dhillon, IS and Campbell, J. Geneva: World Health Organization. https://www.who.int/ hrh/resources/WHO-HLC-Report_web.pdf?ua=1.

Lee, K. and A. Kamradt-Scott. 2014. The multiple meanings of global health governance: a call for conceptual clarity. Globalization and health 10, no 1: 28. 
Lee, K., M. Koivusalo, E. Ollila, R. Labonté, T. Schrecker, C. Schuftan and

D. Woodward. 2007. Globalization, global governance and the social determinants of health: a review of the linkages and agenda for action. Globalization and Health Knowledge Network: Research Papers. WHO Commission on Social Determinants of Health. http://www.who.int/social_ determinants/resources/gkn_lee_al.pdf.

Legge, D.G., C. Schuftan, F.E. Baum, R. van de Pas, D. Sanders, L. Hanson, D. McCoy and A. Sengupta. 2017. Comment-WHO's weakness is not technical, but due to lack of accountability. BMJ global health 2, no Suppl 1: i2-i3.

Lencucha, R. 2013. Cosmopolitanism and foreign policy for health: ethics for and beyond the state. BMC international health and human rights 13, no 1: 29.

Lencucha, R., A. Kothari and R. Labonté. 2010. The role of non-governmental organizations in global health diplomacy: negotiating the Framework Convention on Tobacco Control. Health Policy and Planning 26, no 5: 405-12.

Leschhorn, M., R. van de Pas and T. Schwarz. 2016. Health cooperation: its relevance, legitimacy and effectiveness as a contribution to achieving universal access to health. MMI discussion paper. https://www. medicusmundi.org/contributions/reports/2016/health-cooperation-itsrelevance-legitimacy-and-effectiveness-as-a-contribution-to-achievinguniversal-access-to-health.-mmi-discussion-paper.

Lippmann, W. 1922. Public Opinion. New York: Harcourt.

Liu, J.X., Y. Goryakin, A. Maeda, T. Bruckner and R. Scheffler. 2017. Global health workforce labor market projections for 2030. Human Resources for Health 15, no 1: 11.

Lloyd-Sherlock, P.G., S. Ebrahim, M. McKee and M.J. Prince. 2016. Institutional ageism in global health policy. bmj 354: i4514.

Lucio, R., R. López, N. Leines and J.A. Terán. 2019. El Financiamiento de la Salud en Ecuador. Revista PUCE, no 108.

Mackey, T.K. and B.A. Liang. 2012. Rebalancing brain drain: exploring resource reallocation to address health worker migration and promote global health. Health policy 107, no 1: 66-73.

Mackey, T.K. and B.A. Liang. 2013. Restructuring brain drain: strengthening governance and financing for health worker migration. Global Health Action 6, no 1: 19923.

Mahbubani, K. 2013. The great convergence: Asia, the West, and the logic of one world. New York: Public Affairs.

Mans, L., M. Milicivic, R. van de Pas and H. Guldemann. 2020. Health Workforce Mobility and the European Principle of Solidarity in Health Systems: Open Society Foundations

Mason, P. 2015. The end of capitalism has begun. Guardian. 17 July 2015. https://www. theguardian.com/books/2015/jul/17/postcapitalismend-of-capitalism-begun. 
Mason, P. 2016. Postcapitalism: A guide to our future: Macmillan.

McCollum, R., W. Gomez, S. Theobald and M. Taegtmeyer. 2016. How equitable are community health worker programmes and which programme features influence equity of community health worker services? A systematic review. BMC public health 16, no 1: 419.

McCoy, D. 2017. Critical Global Health: Responding to Poverty, Inequality and Climate Change Comment on "Politics, Power, Poverty and Global Health: Systems and Frames”. Int J Health Policy Manag 6, no 9: 539-41.

McCoy, D., S. Bennett, S. Witter, B. Pond, B. Baker, J. Gow, S. Chand, T. Ensor and B. McPake. 2008a. Salaries and incomes of health workers in sub-Saharan Africa. The Lancet 371, no 9613: 675-81.

McCoy, D., B. McPake and V. Mwapasa. 2008b. The double burden of human resource and HIV crises: a case study of Malawi. Human Resources for Health 6 , no 1: 16.

McFarlane, R.O. 2015. Patterns of Ecological Change and Emerging Infectious Disease in the Australasian Region. In Health of People, Places and Planet, eds Butler, C, Dixon, J and Capon, A: ANU Press.461-76. https://press.anu.edu. $\mathrm{au} /$ publications/health-people-places-and-planet

McInnes, C., A. Kamradt-Scott, K. Lee, D. Reubi, A. Roemer-Mahler, S. Rushton, O.D. Williams and M. Woodling. 2012. Framing global health: the governance challenge. Global Public Health 7, no sup2: S83-S94.

McInnes, C. and S. Rushton. 2014. Health for health's sake, winning for God's sake: US Global Health Diplomacy and smart power in Iraq and Afghanistan. Review of International Studies 40, no 5: 835-57.

McIntyre, D., F. Meheus and J.-A. Røttingen. 2017. What level of domestic government health expenditure should we aspire to for universal health coverage? Health Economics, Policy and Law 12, no 2: 125-37.

McMichael, A.J., K.R. Smith and G.F. Corvalan. 2000. The sustainability transition: a new challenge. Bulletin of the World Health Organization 78, no 9: 1067.

McNeill, D. and O.P. Ottersen. 2015. Global Governance for Health: how to motivate political change? public health 129, no 7: 833-7.

McPake, B., A. Maeda, E.C. Araújo, C. Lemiere, A. El Maghraby and G. Cometto. 2013. Why do health labour market forces matter? Bulletin of the World Health Organization 91, no 11: 841-46.

Meadows, D.H., D.L. Meadows, J. Randers and W.W. Behrens. 1972. The limits to growth. New York 102: 27.

Medecins Sans Frontieres. 2017. Not A Target http://notatarget.msf.org/.

Medico International. 2011. Time to untie the knots: the WHO reform and the need for democratizing global health. The Delhi statement. . https://www. medico.de/en/time-to-untie-the-knots-the-who-reform-and-the-need-fordemocratizing-global-health-15414/. 
Medicus Mundi. 2012. Democratizing global health coalition on the WHO reform (DGH) core statement. https://www.medicusmundi.org/en/topics/pnfpsector-and-global-health-initiatives/who-reform/dgh_core-statement_final. pdf.

Meheus, F. and D. McIntyre. 2017. Fiscal space for domestic funding of health and other social services. Health Economics, Policy and Law 12, no 2: 159-77.

Mestrum, F. 2016. The Social Commons: Rethinking Social Justice in Post-Neoliberal Societies. Petaling Jaya, Malaysia: Gerakbudaya Digital Sdn Bhd.

Meurs, M., L. Seidelmann and M. Koutsoumpa. 2019. How healthy is a 'healthy economy'? Incompatibility between current pathways towards SDG3 and SDG8. Globalization and health 15, no 1: 1-13.

Milanovic, B. 2016a. Global inequality: A new approach for the age of globalization: Harvard University Press.

Milanovic, B. 2016b. What Next? Ten Short Reflections on the Future of Income Inequality and Globalization. In Global inequality, ed. Milanovic, B, Cambridge, MA: Harvard University Press.212-30

Milanovic, B. 2017. The illusion of "degrowth" in a poor and unequal world. Global Inequality Blog. 18 November 2017. http://glineq.blogspot.com/2017/11/theillusion-of-degrowth-in-poor-and.html.

Mills, D., M. Gilbert and B. Wispelwey. 2019. Gaza's Great March of Return: humanitarian emergency and the silence of international health professionals. BMJ global health 4, no 3: e001673.

Mills, E.J., S. Kanters, A. Hagopian, N. Bansback, J. Nachega, M. Alberton, C.G. AuYeung, A. Mtambo, I.L. Bourgeault, S. Luboga, R.S. Hogg and N. Ford. 2011. The financial cost of doctors emigrating from sub-Saharan Africa: human capital analysis. bmj 343: d7031.

Ministère de la Fonction Publique. 2016. de la Réforme de l'Etat et de la Modernisation de l'Administration, Guinée, August 2016

Ministère de la Sante de Guinée. 2016. Consultation intersectorielle des decideurs et des partenaires des pays francophones. Note de la position du pays. WHO. Abidjan, Côte- d'Ivoire, 15 et 16 juin 2016. https://www.who.int/hrh/comheeg/consultation_intersectorielle/fr/.

Ministère de la Sante de Guinée. 2017. Ressources humaines en Santé maternelle et néonatale en Guinée, 2014-2023.

Ministère de la Santé de Guinée. 2015a. Plan de Relance et de Résilience du système de Santé 2015-2017.

Ministère de la Santé de Guinée. 2015b. Plan National de Developpement Sanitaire. Ministère de la Santé de Guinée. 2015c. Strategie de Financement de la Sante vers la couverture sanitaire universelle en Guinee.

Ministère de la Santé de Guinée. 2016. Note de service portant termes de reference de l'Agent Communautaire de Sante (ACS). 
Missoni, E. 2011. WHO reform: threats and opportunities. A healthier political functioning. Bulletin of Medicus Mundi Switzerland No. 122. https://plone. medicusmundi.ch/de/bulletin/mms-bulletin/gesundheitsversorgung-infragilen-staaten/debatte/a-healthier-political-functioning.

Missoni, E. 2013. Understanding the impact of global trade liberalization on health systems pursuing universal health coverage. Value in Health 16, no 1: S14-S18.

Missoni, E. 2015. Degrowth and health: local action should be linked to global policies and governance for health. Sustainability Science 10, no 3: 439-50.

Montes, M.F. 2016. Five Points on the Addis Ababa Action Agenda. South Centre Policy Brief 24 .

Moon, S. 2019. Power in global governance: an expanded typology from global health. Globalization and health 15, no 1: 74.

Moon, S., D. Sridhar, M.A. Pate, A.K. Jha, C. Clinton, S. Delaunay, V. Edwin, M. Fallah, D.P. Fidler and L. Garrett. 2015. Will Ebola change the game? Ten essential reforms before the next pandemic. The report of the HarvardLSHTM Independent Panel on the Global Response to Ebola. The Lancet 386, no 10009: 2204-21.

Moon, S., N.A. Szlezak, C.M. Michaud, D.T. Jamison, G.T. Keusch, W.C. Clark and B.R. Bloom. 2010. The global health system: lessons for a stronger institutional framework. PLoS Med 7, no 1: el000193.

Morrisson, S.J. 2018. The new Barbarianism. https://www.csis.org/features/newbarbarianism

Mullaney, A. and S.A. Hassan. 2015. He Led the CIA to bin Laden-and Unwittingly Fueled a Vaccine Backlash. National Geographic.

Murray, C.J.L., T. Vos, R. Lozano, M. Naghavi, A.D. Flaxman, C. Michaud, M. Ezzati, K. Shibuya, J.A. Salomon, S. Abdalla, V. Aboyans, J. Abraham, I. Ackerman, R. Aggarwal, S.Y. Ahn, M.K. Ali, M. Alvarado, H.R. Anderson, L.M. Anderson, K.G. Andrews, C. Atkinson, L.M. Baddour, A.N. Bahalim, S. Barker-

Collo, L.H. Barrero, D.H. Bartels, M.-G. Basanez, A. Baxter, M.L. Bell, E.J. Benjamin, D. Bennett, E. Bernabe, K. Bhalla, B. Bhandari, B. Bikbov, A. Bin Abdulhak, G. Birbeck, J.A. Black, H. Blencowe, J.D. Blore, F. Blyth, I. Bolliger, A. Bonaventure, S. Boufous, R. Bourne, M. Boussinesq, T. Braithwaite, C. Brayne, L. Bridgett, S. Brooker, P. Brooks, T.S. Brugha, C. Bryan-Hancock, C. Bucello, R. Buchbinder, G. Buckle, C.M. Budke, M. Burch, P. Burney, R. Burstein, B. Calabria, B. Campbell, C.E. Canter, H. Carabin, J. Carapetis, L. Carmona, C. Cella, F. Charlson, H. Chen, A.T.-A. Cheng, D. Chou, S.S. Chugh, L.E. Coffeng, S.D. Colan, S. Colquhoun, K.E. Colson, J. Condon, M.D. Connor, L.T. Cooper, M. Corriere, M. Cortinovis, K.C. de Vaccaro, W. Couser, B.C. Cowie, M.H. Criqui, M. Cross, K.C. Dabhadkar, M. Dahiya, N. Dahodwala, J. Damsere-Derry, G. Danaei, A. Davis, D. De Leo, L. Degenhardt, R. Dellavalle, A. Delossantos, J. Denenberg, S. Derrett, D.C. Des Jarlais, 
S.D. Dharmaratne, et al. 2012. Disability-adjusted life years (DALYs) for 291 diseases and injuries in 21 regions, 1990-2010: a systematic analysis for the Global Burden of Disease Study 2010. The Lancet 380, no 9859: 2197-223.

Nagel, T. 2005. The Problem of Global Justice. Philosophy \& Public Affairs 33, no 2: 113-47. National Health Commission Office of Thailand. The National Health Assembly. https://en.nationalhealth.or.th/nha/.

Neocleous, M. 2015. Der Resilienzdiskurs in der Politik und in der Hilfe (Resisting Resilience: Against the Colonization of Political Imagination). 10th year anniversary and symposium of the Medico International foundation, 5 and 6 June 2015. https://www.medico.de/fileadmin/user_upload/media/Neocleous_ Resisting_Resilience.pdf.

Newman, C. 2014. Time to address gender discrimination and inequality in the health workforce. Human Resources for Health 12, no 1: 25.

Nguyen, V.-K. 2019. An Epidemic of Suspicion-Ebola and Violence in the DRC. New England Journal of Medicine.

Norwegian Ministry of Health and Care Services. 2010. Norwegian Ministry of Foreign Affairs. Norwegian WHO strategy. Norway as a member of WHO's Executive Board 2010-2013. https://www.nivel.nl/sites/default/files/ bestanden/Norwegian_WHO_Strategy_2010-2013_engelsk.pdf.

Nunes, J. 2012. Health, politics and security. e-cadernos ces, no 15.

Nunes, J. 2016. Ebola and the production of neglect in global health. Third World Quarterly 37, no 3: 542-56.

Nunes, J. 2017. Doctors Against Borders. The Politics of Fear: Médecins Sans Frontières and the West African Ebola Epidemic: 1.

Nussbaum, M.C. 2011. Creating capabilities: Harvard University Press.

Nyoni, J. 2008. Improving the health workforce in Africa: the weak link. African Journal of Midwifery and Women's Health 2, no 2: 90-95.

OECD/DAC. 2010. DAC report on multilateral aid. Paris: OECD. https://www.oecd. org/dac/aid-architecture/46062737.pdf.

Ognyanova, D. 2014. Mobility of health professionals before and after the 2004 and 2007 enlargements: evidence from the EU PROMeTHEUS project. Buchan, $W$. et al 2.

Ooms, G. 2014. From international health to global health: how to foster a better dialogue between empirical and normative disciplines. BMC international health and human rights 14, no 1: 36.

Ooms, G. 2015a. Global social protection in health. In "To save humanity": what matters most for a healthy future, eds Frenk, J and Hoffman, S, Oxford: Oxford University Press.

Ooms, G. 2015b. Navigating between stealth advocacy and unconscious dogmatism: the challenge of researching the norms, politics and power of global health. International journal of health policy and management 4, no 10: 641. 
Ooms, G., C. Beiersmann, W. Flores, J. Hanefeld, O. Müller, M. Mulumba, T. Ottersen, M. Sarker and A. Jahn. 2017. Synergies and tensions between universal health coverage and global health security: why we need a second 'Maximizing Positive Synergies' initiative. BMJ global health 2, no 1: e000217.

Ooms, G., C. Brolan, N. Eggermont, A. Eide, W. Flores, L. Forman, E.A. Friedman, T. Gebauer, L.O. Gostin, P.S. Hill, S. Hussain, M. McKee, M. Mulumba, F. Siddiqui, D. Sridhar, L. Van Leemput, A. Waris and A. Jahn. 2013. Universal health coverage anchored in the right to health. Bulletin of the World Health Organization 91, no 1: 2-2A.

Ooms, G. and R. Hammonds. 2016. Global constitutionalism, applied to global health governance: uncovering legitimacy deficits and suggesting remedies. Globalization and health 12, no 1: 84 .

Ooms, G., L.A. Latif, A. Waris, G.E. Brolan, R. Hammonds, E.A. Friedman, M. Mulumba and L. Forman. 2014. Is universal health coverage the practical expression of the right to health care? BMC international health and human rights 14, no 1: 3 .

Ooms, G., T. Ottersen, A. Jahn and I.A. Agyepong. 2018. Addressing the fragmentation of global health: the Lancet Commission on synergies between universal health coverage, health security, and health promotion. The Lancet 392, no 10153: 1098-99.

Ortiz, I. 2019. IMF getting serious on social spending (?) Recovery with a human face Email Listserver

Ortiz, I. and M. Cummins. 2019. Austerity: The New Normal. A Renewed Washington Consensus 2010-24.

Ortiz, I., M. Cummins, J. Capaldo and K. Karunanethy. 2015. The decade of adjustment: A review of austerity trends 2010-2020 in 187 countries.

Ostrom, E. 2010. Beyond markets and states: polycentric governance of complex economic systems. American economic review 100, no 3: 641-72.

Ottersen, O.P., J. Dasgupta, C. Blouin, P. Buss, V. Chongsuvivatwong, J. Frenk, S. Fukuda-Parr, B.P. Gawanas, R. Giacaman and J. Gyapong. 2014. The political origins of health inequity: prospects for change. The Lancet 383, no 9917: 63067.

Ottersen, T., R. Elovainio, D.B. Evans, D. McCoy, D. Mcintyre, F. Meheus, S. Moon, G. Ooms and J.-A. Røttingen. 2017. Towards a coherent global framework for health financing: recommendations and recent developments. Health Economics, Policy and Law 12, no 2: 285-96.

Pan American Health Organisation. 2005. Toronto Call to Action. 2006 2015 . Towards a decade of Human Resources in Health for the Americas. Regional Meeting of the Observatory of Human Resources in Health, 2005. http:// www.bvsde.paho.org/bvsdeescuelas/fulltext/CallAction_engl.pdf.

Paul, E., L. Albert, B.N.S. Bisala, O. Bodson, E. Bonnet, P. Bossyns, S. Colombo, V. De 
Brouwere, A. Dumont and D.S. Eclou. 2018. Performance-based financing in low-income and middle-income countries: isn't it time for a rethink? BMJ global health 3, no 1: e000664.

Piot, P. 2012. No time to lose: A life in pursuit of deadly viruses. New York: W. W. Norton.

Pogge, T. 2004. Relational conceptions of justice: Responsibilities for health outcomes.

Pogge, T. 2005a. Human Rights and Global Health: A Research Program. Metaphilosophy 36, no 1-2: 182-209.

Pogge, T. 2005b. World poverty and human rights. Ethics \& international affairs 19, no 1: 1-7.

Pope, A., H.A. Higginbottom, G. Smith and T. Frieden. 2016. A Path to Global Health Security. The White House Blog. October 12, 2016. https://obamawhitehouse. archives.gov/blog/2016/10/12/path-global-health-security.

Pope Francis. 2015. Encyclical letter 'Laudato Si' of the Holy Father Francis: On care for our common home. https://laudatosi.com/watch.

Prashanth, N.S., B. Marchal, N. Devadasan, G. Kegels and B. Criel. 2014. Advancing the application of systems thinking in health: a realist evaluation of a capacity building programme for district managers in Tumkur, India. Health research policy and systems 12, no 1: 42 .

Pronk, J. 2015. Op zoek naar een nieuwe kaart. Verspreide aantekeningen over ontwikkeling en ontwikkelingssamenwerking (Charting a new path. Scattered notes on development and development cooperation). Volendam: LM Publishers.

Purdy, J. 2015. After Nature: A Politics for the Anthropocene. Cambridge, MA: Harvard University Press.

Rajkotia, Y. 2018. Beware of the success cartel: a plea for rational progress in global health. BMJ global health 3, no 6: e001197.

Ravelo, J.L. 2016. How should we measure access to health care? Devex. https:// www.devex.com/news/how-should-we-measure-access-to-healthcare-87862.

Raworth, K. 2017a. Doughnut Economics: Seven Ways to Think Like a 21st-Century Economist. Vermont: Chelsea Green Publishing.

Raworth, K. 2017b. A Doughnut for the Anthropocene: humanity's compass in the 21st century. The lancet planetary health 1, no 2: e48-e49.

Reddi, A., A. Thyssen, D. Smith, J.H. Lange and C. Akileswaran. 2012. Human capital contracts for global health: a plan to increase the number of physicians in resource limited settings. Aids 26, no 15: 1979-80.

Reddy, S.K., S. Mazhar and R. Lencucha. 2018. The financial sustainability of the World Health Organization and the political economy of global health governance: a review of funding proposals. Globalization and health 14, no 1: 119.

Rees, S.J., R. van de Pas, D. Silove and M. Kareth. 2008. Health and human security in West Papua. Medical journal of Australia 189, no 11-12: 641-43. 
Reich, M.R. 2019. Political economy analysis for health. Editorial. Bulletin of the World Health Organization 97, no 8.

Research and Degrowth. 2016. Research and actions to consume less and share more. http://www.degrowth.org.

Resilience Journal. Aims and Scope. Taylor \& Francis website. http://www.tandfonline.com/action/ journalInformation?show=aimsScope\&journalCode=resi20.

Rodin, J. 2013. The Resilience Dividend: Being Strong in a World Where Things Go Wrong. New York: Public Affairs.

Rodrik, D. 201la. The Globalization Paradox: Democracy and the Future of the World Economy. New York: WW Norton \& Company.

Rodrik, D. 2011b. The Globalization Paradox: Why Global Markets, States, and Democracy Can't Coexist. New York: Oxford University Press.

Rodrik, D. 2017. Straight talk on trade: Ideas for a sane world economy: Princeton University Press.

Rodrik, D. 2018. Globalisation: New Deal on Labour Mobility. Social Europe. 26 April 2018. https://www.socialeurope.eu/globalisation-new-deal-on-labourmobility.

Roemer-Mahler, A. and S. Elbe. 2016. The race for Ebola drugs: pharmaceuticals, security and global health governance. Third World Quarterly 37, no 3: 487-506.

Rosling, H. 2019. Factfulness: Flammarion.

Rowden, R. 2014. West Africa's financial immune deficiency. Foreign Policy. https:// foreignpolicy.com/2014/10/30/west-africas-financial-immune-deficiency/.

Rowden, R. 2019 Advocates of the SDGs have a monetarism problem. Sheffield Political Economy Research Institute

Rubinstein, A., M.C. Zerbino, C. Cejas and A. López. 2018. Making universal health care effective in Argentina: a blueprint for reform. Health Systems \& Reform 4 , no 3: 203-13.

Ruckert, A., R. Labonté, R. Lencucha, V. Runnels and M. Gagnon. 2016. Global health diplomacy: a critical review of the literature. Social Science \& Medicine 155: 61-72.

Rudan, I. and K.Y. Chan. 2015. Global health metrics needs collaboration and competition. The Lancet 385, no 9963: 92-4.

Ruger, J.P. 2010. Health capability: conceptualization and operationalization. Am J Public Health 100, no 1: 41-9.

Ruger, J.P. and D. Yach. 2009. The Global Role of the World Health Organization. Glob Health Gov 2, no 2: 1-11.

Rushton, S. 2011. Global health security: security for whom? Security from what? Political Studies 59, no 4: 779-96.

Rushton, S. and C. McInnes. 2006. The UK, health and peace-building: the mysterious disappearance of Health as a Bridge for Peace. Medicine, Conflict 
and Survival 22, no 02: 94-109.

Rushton, S. and O.D. Williams. 2012. Frames, paradigms and power: global health policy-making under neoliberalism. Global Society 26, no 2: 147-67.

Russo, G., G. Bloom and D. McCoy. 2017. Universal health coverage, economic slowdown and system resilience: Africa's policy dilemma. BMJ global health 2, no 3: e000400.

Russo, G., L. Xu, M. McIsaac, M.D. Matsika-Claquin, I. Dhillon, B. McPake and J. Campbell. 2019. Health workers' strikes in low-income countries: the available evidence. Bulletin of the World Health Organization 97, no 7: 460.

Sachs, J.D. 2019. Financing UHC in Low-Income Countries. In Health: A political choice; Delivering Universal Health Coverage 2030, eds Kirton, J and Kickbusch, I: The Global Governance Project.

Said, E. 2014. Orientalism. In Geopolitics: Routledge.75-79

SAPRIN. 2004. Structural adjustment: The SAPRI report-the policy roots of economic crisis, poverty and inequality. London: Zed Books.

Savage, M. 2019. NHS winter crisis fears grow after thousands of EU staff quit. The Guardian

Scheil-Adlung, X. 2013. Health workforce benchmarks for universal health coverage and sustainable development. Bull World Health Organ 91: 888-88.

Schinkel, W. and R. van Reekum. 2019. Theorie van de Kraal: Boom.

Scholte, J.A. 2004. Globalization and governance: from statism to polycentrism. Centre for the study of Globalisation and Regionalisation Working Paper. No 130/04; February 2004. http://wrap.warwick.ac.uk/1984/1/WRAP_Scholte_ wp13004.pdf.

Schrecker, T. 2012. Interrogating scarcity: how to think about 'resource-scarce settings'. Health Policy and Planning 28, no 4: 400-09.

Schrecker, T. 2017. A New Gilded Age, and What It Means for Global Health: Comment on" Global Health Governance Challenges 2016-Are We Ready?". International journal of health policy and management 6, no 3: 169.

Scott, K., S. Beckham, M. Gross, G. Pariyo, K.D. Rao, G. Cometto and H.B. Perry. 2018. What do we know about community-based health worker programs? A systematic review of existing reviews on community health workers. Human Resources for Health 16, no 1: 39.

Seidman, G. 2017. Does SDG 3 have an adequate theory of change for improving health systems performance? Journal of global health 7, no 1.

Sénit, C.-A., F. Biermann and A. Kalfagianni. 2017. The Representativeness of Global Deliberation: A Critical Assessment of Civil Society Consultations for Sustainable Development. Global Policy 8, no 1: 62-72.

Sheikh, K., H. Schneider, I.A. Agyepong, U. Lehmann and L. Gilson. 2016. Boundary-spanning: reflections on the practices and principles of Global Health. BMJ global health 1, no 1: e000058. 
Shiffman, J. 2014. Knowledge, moral claims and the exercise of power in global health. Int J Health Policy Manag 3, no 6: 297-9.

Shiffman, J. 2015. Global health as a field of power relations: a response to recent commentaries. International journal of health policy and management 4, no 7: 497.

Shiffman, J. 2017. Four challenges that global health networks face. International journal of health policy and management 6 , no 4: 183.

Shiffman, J., H. Peter Schmitz, D. Berlan, S.L. Smith, K. Quissell, U. Gneiting and D. Pelletier. 2016. The emergence and effectiveness of global health networks: findings and future research. Health Policy and Planning 31, no suppl_1: illo-i23.

Siddiqui, F.R., E.A. Friedman and A. Nader. 2014. Representativeness of the UN post-2015 national consultations. The Lancet Global Health 2, no 11: e631-2.

Sidibé, M. and J. Campbell. 2015. Reversing a global health workforce crisis. Bulletin of the World Health Organization 93, no 1: 3.

Siyam, A., P. Zurn, O.C. Ro, G. Gedik, K. Ronquillo, C. Joan Co, C. VaillancourtLaflamme, J. dela Rosa, G. Perfilieva and M.R. Dal Poz. 2013. Monitoring the implementation of the WHO Global Code of Practice on the International Recruitment of Health Personnel. Bulletin of the World Health Organization 91, no 11: 816-23.

Slaughter, A.-M. 2003. Everyday global governance. Daedalus 132, no 1: 83-90.

Smith, J. 2015. Global health security: a flawed SDG framework. The Lancet 385, no 9984: 2249.

Smith, R.D., R. Beaglehole, D. Woodward and N. Drager. 2003. Global public goods for health: health economic and public health perspectives. Oxford: Oxford University Press.

Smith, R.D. and D. Woodward. 2003. Global Public Goods for Health: Use and Limitations. Trade, foreign policy, diplomacy and health. World Health Organization. https://www.who.int/trade/distance_learning/gpgh/gpgh9/ en/.

Sondorp, E. and O. Bornemisza. 2011. Editorial Health Diplomacy Monitor 2, no 2.

Soucat, A., R. Scheffler and T.A. Ghebreyesus. 2013. The labor market for health workers in Africa: a new look at the crisis. Washington: The World Bank.

Sousa, A., R.M. Scheffler, J. Nyoni and T. Boerma. 2013. A comprehensive health labour market framework for universal health coverage. Bulletin of the World Health Organization 91: 892-94.

Sphere Association. 2018 The Sphere Handbook: Humanitarian Charter and Minimum

Standards in Humanitarian Response, fourth edition. Geneva

Sridhar, D. 2012. Who sets the global health research agenda? The challenge of multi-bi financing. PLoS Med 9, no 9: el001312.

Sridhar, D., C.E. Brolan, S. Durrani, J. Edge, L.O. Gostin, P. Hill and M. McKee. 2013. 
Recent shifts in global governance: implications for the response to noncommunicable diseases. PLoS Med 10, no 7: el001487.

Sridhar, D., M. McKee, G. Ooms, C. Beiersmann, E. Friedman, H. Gouda, P. Hill and A. Jahn. 2015. Universal Health Coverage and the Right to Health: From Legal Principle to Post-2015 Indicators. Int J Health Serv 45, no 3: 495-506.

Sridhar, D. and N. Woods. 2013. Trojan Multilateralism: Global Cooperation in Health. Global Policy 4, no 4: 325-35.

Steffen, W., K. Richardson, J. Rockstrom, S.E. Cornell, I. Fetzer, E.M. Bennett, R. Biggs, S.R. Carpenter, W. de Vries, C.A. de Wit, C. Folke, D. Gerten, J. Heinke, G.M. Mace, L.M. Persson, V. Ramanathan, B. Reyers and S. Sorlin. 2015. Planetary boundaries: guiding human development on a changing planet. Science 347, no 6223: 1259855.

Stein, F. and D. Sridhar. 2019. Back to the future? Health and the World Bank's human capital index. bmj 367.

Stenberg, K., O. Hanssen, T.T.-T. Edejer, M. Bertram, C. Brindley, A. Meshreky, J.E. Rosen, J. Stover, P. Verboom and R. Sanders. 2017. Financing transformative health systems towards achievement of the health Sustainable Development Goals: a model for projected resource needs in 67 low-income and middleincome countries. The Lancet Global Health 5, no 9: e875-e87.

Steurs, L., R. van de Pas, S. Delputte and J. Orbie. 2018. The Global Health policies of the EU and its Member States: a common vision? International journal of health policy and management 7, no 5: 433.

Stiglitz, J.E. 2002. Globalization and its discontents. New York: W. W. Norton.

Stiglitz, J.E., A. Sen and J.-P. Fitoussi. 2010. Mismeasuring Our Lives: Why GDP Doesn't Add Up. New York: The New Press.

Stone, D.A. 1988. Policy paradox and political reason: Scott Foresman \& Co.

Storeng, K.T. and J. Palmer. 2019. When ethics and politics collide in donor-funded global health research. The Lancet 394, no 10193: 184-86.

Stubbs, T., A. Kentikelenis, D. Stuckler, M. McKee and L. King. 2017. The impact of IMF conditionality on government health expenditure: A cross-national analysis of 16 West African nations. Social Science \& Medicine 174: 220-27.

Stuckler, D. and S. Basu. 2013. The body economic: why austerity kills: Basic Books (AZ).

Stuckler, D., S. Basu and M. McKee. 2011. Global health philanthropy and institutional relationships: how should conflicts of interest be addressed? PLoS Med 8, no 4: el001020.

Stuckler, D. and M. McKee. 2008. Five metaphors about global-health policy. The Lancet 372, no 9633: 95-97.

Sweden. 2011. Sweden's strategy for WHO 2011-2015. Swedish Ministry of Health and Social Affairs. Regeringskansliet, S2011/3711/EIS. https://www. government.se/49b757/contentassets/19faae4e59e547e09f15c438fbd4bc20/ swedens-strategy-for-who-2011-2015-s2011371leis. 
Sweijs, T. and D. Pronk. 2019. Interregnum. Strategische Monitor 2018-201:

Netherlands Institute of International Relations 'Clingendael'.

Tankwanchi, A.B.S., S.H. Vermund and D.D. Perkins. 2014. Has the WHO Global

Code of Practice on the International Recruitment of Health Personnel been effective? The Lancet Global Health 2, no 7: e390-e91.

Taylor, A.L. and I.S. Dhillon. 2011. The WHO Global Code of Practice on the International Recruitment of Health Personnel: The Evolution of Global Health Diplomacy. Global Health Governance (Fall 2011). http://blogs.shu. edu/ghg/files/2011/11/Taylor-and-Dhillon_The-WHO-Global-Code-ofPractice-on-the-International-Recruitment-of-Health-Personnel_Fall-2011. pdf.

Tcherneva, P. 2018. The Job Guarantee: Design, Jobs, and Implementation. Levy Economics Institute. https://EconPapers.repec.org/ RePEc:lev:wrkpap:wp_902.

The Graduate Institute Geneva. 2016. Global Health Centre. How to make the global health governance system fit for the 21st century? Policy Dialogue Workshop of the SNIS-funded research project on 'How to Break the Gridlock in Global Health Governance. https:/graduateinstitute.ch/ communications/news/how-make-global-health-governance-system-fit21st-century.

The Guardian. 2014a. Canada suspends visas for residents of west African Ebola outbreak countries. (31 October 2014). www.theguardian.com/world/2014/ oct/31/canada-ebola-suspends-visas-west-africa.

The Guardian. 2014b. Cut in UK aid to Sierra Leone may have helped spread of Ebola, MPs say. (2 October 2014). https://www.theguardian.com/world/2014/ oct/02/ebola-conference-london-hammond-sierra-leone.

The Lancet - Editorial. 2016. No health workforce, no global health security. The Lancet 387.10033 2016: 2063. https://doi.org/10.1016/S0140-6736(16)30598-0.

The White Helmets. 2018. https://www.whitehelmets.org/en

Third Global Forum on Human Resources for Health. 2013. HRH commitments www.who.int/workforcealliance/forum/2013/hrh_commitments/en/index. html

Thomson, S., J. Figueras, T. Evetovits, M. Jowett, P. Mladovsky, A. Maresso, J. Cylus, M. Karanikolos and H. Kluge. 2014. Policy summary 12. Economic crisis, health systems and health in Europe: impact and implications for policy. Copenhagen: WHO Regional Office for Europe and European Observatory on Health Systems and Policies. http://www.euro.who.int/_data/assets/ pdf_file/0008/257579/Economic-crisis-health-systems-Europe-impactimplications-policy.pdf.

Tjadens, F., C. Weilandt and J. Eckert. 2013. Mobility of Health Professionals - health systems, work conditions, patterns of health workers' mobility and implications for 
policy makers. New York: Springer.

Turner, G. 2014. Is global collapse imminent? An updated comparison of the Limits to Growth with historical data. MSSI Research paper 4. Melbourne Sustainable Society Institute, The University of Melbourne. https:// sustainable.unimelb.edu.au/_data/assets/pdf_file/0005/2763500/MSSIResearchPaper-4_Turner_2014.pdf.

UK Department of Health. 2001. Code of practice for NHS employers involved in international recruitment of healthcare professionals. London: Department of Health.

UN Sustainable Development Knowledge Platform. 2015. Sustainable Development Goal 3. Ensure healthy lives and promote well-being for all at all ages. https://www.un.org/sustainabledevelopment/health/.

United Nations. 2015a. Addis Ababa Action Agenda of the Third International Conference on Financing for Development; July 13-16, 2015; Addis Ababa, Ethiopia. https://www.un.org/esa/ffd/wp-content/uploads/2015/08/AAAA Outcome.pdf.

United Nations. 2015b. Sustainable Development Goals. Goal 17: Revitalize the global partnership for sustainable development. http://www.un.org/ sustainabledevelopment/globalpartnerships.

United Nations Environment Programme. 1992. Rio Declaration on Environment and Development. http://wedocs.unep.org/bitstream/ handle/20.500.11822/19163/Rio_Declaration_on_Environment_and_ Development.pdf?sequence=1\&isAllowed=y.

United Nations General Assembly. 2015. Addis Ababa Action Agenda of the Third International Conference on Financing for Development (Addis Ababa Action Agenda). In A/RES/69/313, ed. Nations, U. New York.

United Nations General Assembly (UNGA). 2014. Measures to contain and combat the recent Ebola outbreak in West Africa. Resolution 69/1 adopted by the General Assembly on 19 September. https:/www.un.org/en/ga/search/view_ doc.asp?symbol=A/RES/69/1.

United Nations Security Council. 2000. The Responsibility of the Security Council in the Maintenance of International Peace and Security: HIV/AIDS and International Peace-keeping Operations. Resolution 1308 United Nations Security Council

United Nations Security Council. 2014. Resolution 2177. S/RES/2177. 18 September 2014. http://undocs.org/S/RES/2177\%20(2014).

United Nations Security Council. 2016. Strongly Condemning Attacks against Medical Facilities, Personnel in Conflict Situations. In Resolution 2286 Security Council 7685th Meeting (AM) SC/12347.

UNOCHA. 2019. Global Humanitarian Overview 2019. 
van Belle, S., R. van de Pas and B. Marchal. 2018. Queen bee in a beehive: WHO as meta-governor in global health governance. BMJ global health 3, no 1: e000448.

van de Pas, R. 2010. Human resources for health, opportunities and challenges in the Indonesian province of Papua.

van de Pas, R. 2015. The health workforce as a crucial bottleneck in containing Ebola. In International Health Policies Network Institute of Tropical Medicine, Antwerp

van de Pas, R. 2016. Global Health in the Anthropocene: Moving Beyond Resilience and Capitalism Comment on "Health Promotion in an Age of Normative Equity and Rampant Inequality". International Journal of Health and Policy Management 6: 1-6.

van de Pas, R. 2017. Global health in the anthropocene: moving beyond resilience and capitalism: comment on" health promotion in an age of normative equity and rampant inequality". International journal of health policy and management 6, no 8: 481.

van de Pas, R. 2019. The Universal Health Coverage Divide. https://www. internationalhealthpolicies.org/blogs/the-universal-health-coverage-divide/ (accessed.

van de Pas, R., P.S. Hill, R. Hammonds, G. Ooms, L. Forman, A. Waris, C.E. Brolan, M. McKee and D. Sridhar. 2017a. Global health governance in the sustainable development goals: Is it grounded in the right to health? Global Challenges 1, no 1: 47-60.

Van de Pas, R. and L. Mans. 2018. Global Skills Partnerships \& Health Workforce Mobility: pursuing a race to the bottom? : Public Services International van de Pas, R. and L. Mans. 2019. Global Skills Partnerships \& Health Workforce Mobility: Pursuing a race to the bottom?: Public Services International van de Pas, R., L. Mans, M. Bemelmans and A. Krumeich. 2018. Framing the health workforce agenda beyond economic growth. International journal of health policy and management 7, no 8: 678.

van de Pas, R., L. Mans, G. de Ponte and Y. Dambisya. 2016. The Code of Practice and its enduring relevance in Europe and Eastern and Southern Africa. Human Resources for Health 14, no 1: 30.

van de Pas, R. and S. van Belle. 2015. Ebola, the epidemic that should never have happened. Global Affairs 1, no 1: 95-100.

Van de Pas, R. and L.G. Van Schaik. 2014. Democratizing the world health organization. Public Health 128, no 2: 195-201.

van de Pas, R., A. Veenstra, D. Gulati, W. van Damme and G. Cometto. 2017b. Tracing the policy implementation of commitments made by national governments and other entities at the Third Global Forum on Human Resources for Health. BMJ global health 2, no 4: e000456. 
van Ham, P. 2013. Transnational Governance and Democratic Legitimacy-A Conceptual Overview. The Hague Institute for Global Justice and the Netherlands Institute of International Relations' Clingendael.

van Ham, P., R. van de Pas, L.G. van Schaik and J. Rood. 2013. Transnational Governance and Democratic Legitimacy - A Conceptual Overview. The Hague Institute for Global Justice and the Netherlands Institute of International Relations 'Clingendael'.

van Schaik, L.G. and S. Battams. 2014a. The EU's role in creating a more effective WHO. In The EU and effective multilateralism: Internal and external reform practices, eds Van Schaik, LG and Drieskens, E, London: Routledge.p. 33-48

van Schaik, L.G. and S. Battams. 2014b. The EU's role playing in the act of a more effective World Health Organisation. In: Drieskens E, van Schaik LG, editors. The EU Effective Multilateralism: internal and external reform practices. London and New York: Routledge.

Vandemoortele, J. 2015. Tackling Inequality is Key to the Post-MDGs Development Agenda. Europe's world, Brussels, Belgium.

Verma, A.A. 2017. Failing to protect humanitarian workers: lessons from Britain and Voluntary Aid Detachments in the Second World War. Medicine, Conflict and Survival 33, no 3: 216-28.

The White Helmets. Directed by Von Einsiedel, O. Netflix, 2016.

Voss, M., R. Marten and D. Gulati. 2019. Accelerating the SDG3 Global Action Plan: BMJ Specialist Journals.

Vujicic, M., K. Ohiri and S.P. Sparkes. 2009. Working in health: financing and managing the public sector health workforce. Washington: The World Bank.

Wallace, R.G., M. Gilbert, R. Wallace, C. Pittiglio, R. Mattioli and R. Kock. 2014. Did Ebola Emerge in West Africa by a Policy-Driven Phase Change in Agroecology? Ebola's Social Context. Environment and Planning A: Economy and Space 46, no 11: 2533-42.

Walt, G. and L. Gilson. 1994. Reforming the health sector in developing countries: the central role of policy analysis. Health Policy and Planning 9, no 4: 353-70.

Wemos. 2019. Factsheet: Fiscal space for health and four ways to increase it.

Wenham, C., R. Katz, C. Birungi, L. Boden, M. Eccleston-Turner, L. Gostin, R. Guinto, M. Hellowell, K.H. Onarheim and J. Hutton. 2019. Global health security and universal health coverage: from a marriage of convenience to a strategic, effective partnership. BMJ global health 4, no 1: e001145.

Wetzels, H. 2019. Europa moet niet weten van revolutionair nieuw VN-verdrag: Mondiaal Nieuws

WHO, ILO and OECD. 2017. Working for Health 
WHO Executive Board. 2015. Ebola: ending the current outbreak, strengthening global preparedness and ensuring WHO's capacity to prepare for and respond to future large scale outbreaks and emergencies with health consequences. EBSS3.R1. Special session on Ebola. http://apps.who.int/gb/ ebwha/pdf_files/EBSS3/EBSS3_Rl-en.pdf?ua=1\&ua=1.

WHO Regional Office for Europe. 2013. Health systems and economic crisis in Europe. Impact and policy implications.

Willetts, A. and T. Martineau. 2004. Ethical international recruitment of health professionals: will codes of practice protect developing country health systems. Liverpool School of Tropical Medicine. https://assets. aspeninstitute.org/content/uploads/files/content/images/Martineau\%20 codesofpracticereport.pdf.

Williams, C. and A. Blaiklock. 2016. Human Rights Discourse in the Sustainable Development Agenda Avoids Obligations and Entitlements Comment on "Rights Language in the Sustainable Development Agenda: Has Right to Health Discourse and Norms Shaped Health Goals?". Int J Health Policy Manag 5, no 6: 387-90.

Williams, O.D., G. Ooms and P.S. Hill. 2015. Cautionary Notes on a Global Tiered Pricing Framework for Medicines. Am J Public Health 105, no 7: 1290-3.

Wintour, P. 2018. UK agrees to take in some White Helmets evacuated from Syria by Israel The Guardian

Witter, S., M.P. Bertone, Y. Chirwa, J. Namakula, S. So and H.R. Wurie. 2016. Evolution of policies on human resources for health: opportunities and constraints in four post-conflict and post-crisis settings. Confl Health 10: 31.

Witter, S. and B. Hunter. 2017. How do different types of provider affect access to effective and affordable healthcare during and after crises? Health systems during and after crisis: evidence for better policy and practice. Brief 4. ReBUILD consortium. June 2017. https://rebuildconsortium.com/ media/1538/rebuild_briefing_4_june_17_providers.pdf.

Wolfe, N. 2013. The viral storm: The dawn of a new pandemic age. London: Penguin Books.

Woodward, D. 2015. Incrementum ad absurdum: global growth, inequality and poverty eradication in a carbon-constrained world. World Econ Rev 4: 43-62.

World Bank Group. 2019. The changing nature of work http://documents. worldbank.org/curated/en/816281518818814423/pdf/2019-WDR-Report.pdf.

World Health Organization. 1946. Constitution of the World Health Organization. International Health Conference, New York. http://apps.who.int/gb/bd/PDF/ bd47/EN/constitution-en.pdf.

World Health Organization. 1997. A New global health policy for the twentyfirst century : an NGO perspective : outcome of a Formal Consultation with Nongovernmental Organizations held at WHO Geneva 2 and 3 May 
1997. https://apps.who.int/iris/bitstream/handle/10665/63511/WHO_PPE_ PAC_97.3.pdf?sequence=1.

World Health Organization. 1998. Health for all in the 21st Century: World Health Organization.

World Health Organization. 2002. Principles governing relations with nongovernmental organizations. Geneva: Givil Society Initiative. https:// www.who.int/governance/civilsociety/principles/en/.

World Health Organization. 2004a. Fifty-Seventh World Health Assembly. International migration of health personnel: a challenge for health systems in developing countries. WHA57.19. http://apps.who.int/gb/ebwha/pdf_files/ WHA57/A57_R19-en.pdf.

World Health Organization. 2004b. Policy for relations with nongovernmental organizations. 57th World Health Assembly. A57/32. http://apps.who.int/gb/ ebwha/pdf_files/WHA57/A57_REC1-en.pdf.

World Health Organization. 2005. International Health Regulations. Part VIII General provisions: p.42-6

World Health Organization. 2006. World Health Report 2006 - Working Together for Health. World Health Organization, Geneva. https://www.who.int/ whr/2006/whr06_en.pdf.

World Health Organization. 2007. The world health report 2007: a safer future: global public health security in the 21st century.

World Health Organization. 2008. The Kampala declaration and agenda for global action.

World Health Organization. 2010. The future of financing for WHO. Executive Board 128th session. EB 128/21. http://apps.who.int/gb/ebwha/pdf_files/ EB128/B128_21-en.pdf.

World Health Organization. 2011a. The future of financing for WHO. World Health Organization: reforms for a healthy future. 64 th world health assembly. A64/4. https://apps.who.int/gb/ebwha/pdf_files/WHA64/A64_4-en.pdf.

World Health Organization. 2011b. Report of the Review Committee on the Functioning of the International Health Regulations (2005) in relation to Pandemic (H1N1) 2009. In A64/10, ed. Assembly, WH. Geneva.

World Health Organization. 2012a. Country Coordination and Facilitation. Principles and process. http://www.who.int/workforcealliance/knowledge/ resources/CCF_Principles_Processes_web.pdf?ua=1.

World Health Organization. 2012b. Report of the programme, budget and administration committee of the Executive Board at its second extraordinary meeting. Executive Board 132nd session. EB 132/3. https://apps.who.int/iris/ bitstream/handle/10665/78598/B132_3-en.pdf?sequence=1\&isAllowed=y. 
World Health Organization. 2012c. Report of the programme, budget and administration committee of the Executive Board at its second extraordinary meeting. Executive Board 132nd session. EB 132/5. Par. 6. https://apps.who.int/iris/bitstream/handle/10665/78598/B132_3-en. pdf? sequence $=1 \&$ isAllowed $=y$.

World Health Organization. 2012d. Review of management, administration and decentralization in the World Health Organization, report by the Joint Inspection Unit. Executive Board. 132nd session. EB132/5 Add.6. https://apps.who.int/iris/bitstream/handle/10665/78609/B132_5Add6-en. pdf? sequence $=1 \&$ isAllowed $=y$.

World Health Organization. 2012e. WHO Reform: NGO consultation. Consultation on WHO's engagement with NGOs: Issues to consider in the formulation of a policy. https://www.who.int/about/who_reform/governance/who_reform_ consultation_who_ngo_engagement_issues_paper.pdf?ua=1.

World Health Organization. 2013a. The Recife Political Declaration on Human Resources for Health. Renewed commitments towards universal health coverage. 3rd Global Forum on Human Resources for Health. http:// www. who.int/workforcealliance/forum/2013/recife_declaration_17nov.pdf?ua=1

World Health Organization. 2013b. WHO governance reform. Executive Board. 133rd session. EB133/16. https://apps.who.int/gb/ebwha/pdf_files/EB133/ B133_16-en.pdf.

World Health Organization. 2013c. WHO policy dialogue on international health workforce mobility and recruitment challenges: technical report. http:// www.euro.who.int/_data/assets/pdf_file/0007/200698/WHO-policydialogue-on-international-health-workforce-mobility-and-recruitmentchallenges-technical-report.pdf?ua=1.

World Health Organization. 2013d. WHO reform. High-level implementation plan and report. 66th world health assembly. A66/4 . http://apps.who.int/gb/ ebwha/pdf_files/WHA66/A66_49-en.pdf.

World Health Organization. 2013e. WHO's role in global health governance. Executive Board. 132nd session. EB132/5 Add.5. http://apps.who.int/gb/ ebwha/pdf_files/ebl32/b132_5add5-en.pdf.

World Health Organization. 2015a. Report of the Expert Advisory Group on the Relevance and Effectiveness of the WHO Global Code of Practice on the International Recruitment of Health Personnel: Report by the DirectorGeneral.

World Health Organization. 2015b. Workforce 2030 and the sustainable development goals. http://www.who.int/hrh/HRH-SDG_infographic_ Jan2016.png?ua=1.

World Health Organization. 2016a. Global Strategy on Human Resources for Health: Workforce 2030. http://www.who.int/hrh/resources/global_strategy2030en- 
printversion.pdf?ua=1.

World Health Organization. 2016b. High-Level Commission on Health Employment and Economic Growth. Working for health and growth: investing in the health workforce. https://apps.who.int/iris/bitstream/hand le/10665/250047/9789241511308-eng.pdf?sequence=1.

World Health Organization. 2016c. Resolutions of the 69th World Health Assembly, and 138th and 139th Executive Board meetings. World Health Organization. . http://apps.who.int/gb/e/e_wha69.html\#top; http://apps.who.int/gb/e/e_ eb138.html and http://apps.who.int/gb/e/e_ebl39.html.

World Health Organization. 2016d. UN High-Level Commission on Health

Employment and Economic Growth. http://www.who.int/hrh/com-heeg/en/.

World Health Organization. 2016e. Working for health and growth: investing in the health workforce. Report of the High-Level Commission on Health Employment and Economic Growth. https://apps.who.int/iris/bitstream/ha ndle/10665/250047/9789241511308-eng.pdf?sequence=1.

World Health Organization. 2016f. Working for health and growth: investing in the health workforce. Report of the High-Level Commission on Health Employment and Economic Growth. . https://apps.who.int/iris/bitstream/ha ndle/10665/250047/9789241511308-eng.pdf?sequence=1.

World Health Organization. 2016g. World Health Organization. Global strategy on human resources for health: workforce 2030. https://www.who.int/hrh/ resources/global_strategy_workforce2030_14_print.pdf?ua=1.

World Health Organization. 2017a. Dublin Declaration on Human Resources for Health: Building the Health Workforce of the Future.

World Health Organization. 2017b. Fourth Global Forum on Human Resources for Health. Dublin Declaration on Human Resources for Health: Building the Health Workforce of the future. https:/www.who.int/hrh/events/Dublin Declaration-on-HumanResources-for-Health.pdf.

World Health Organization. 2017c. Framing the health workforce agenda for the Sustainable Development Goals: biennium report 2016-2017 - WHO Health Workforce. https://www.who.int/hrh/BienniumReportRevised2017.pdf.

World Health Organization. 2018a. Five-year action plan for health employment and inclusive economic growth (2017-2021).

World Health Organization. 2018b. International Platform on Health Worker Mobility :Evidence, Solutions and Instruments. meeting report. Geneva: ILO, OECD \& WHO

World Health Organization. 2018c. International Platform on Health Worker Mobility: Evidence, Solutions and Instruments.

World Health Organization. 2018d. International trade in services \& health worker mobility: an examination. Draft working paper for comment. In Human resources for health Oberver Series. No. 24. 
World Health Organization. 2018e. Title. no.24.

World Health Organization. 2018f. Surveillance System for Attacks on Health Care https://publicspace.who.int/sites/ssa/SitePages/PublicDashboard.aspx.

World Health Organization. 2018g. Thirteenth general programme of work 20192023. The seventy-first world health assembly. Geneva (Switzerland): World Health Organization.

World Health Organization. 2018h. WHO calls for protection of humanitarian workers and civilians in Democratic Republic of the Congo. . http://www. who.int/news-room/detail/26-09-2018-who-calls-for-protection-ofhumanitarian-workers-and-civilians-in--democratic-republic-of-thecongo

World Health Organization. 2018 Welcome to the WHO Emergency Medical Teams Initiative. https://extranet.who.int/emt/

World Health Organization. 2019a. Delivered by women, led by men: A gender and equity analysis of the global health and social workforce. Geneva: World Health Organization; 2019 (Human Resources for Health Observer Series No. 24). Licence: CC BY-NC-SA 3.0 IGO. https://apps.who.int/iris/bitstream/han dle/10665/311322/9789241515467-eng.pdf.

World Health Organization. 2019b. Global Health Expenditure Database.

World Health Organization. 2019c. Global Symposium on Health Workforce Accreditation and Regulation. Istanbul, Turkey

World Health Organization. 2019d. National Health Workforce Accounts

Surveillance System for Attacks on Health Care https://publicspace.who.int/sites/ ssa/SitePages/PublicDashboard.aspx.

World Health Organization. 2019f. WHO Ebola responder killed in attack on the Butembo hospital Geneva

Yeates, N. and J. Pillinger. 2013. Human Resources for Health Migration: global policy responses, initiatives, and emerging issues. Milton Keynes: University of Ottawa - The Open University.

Yeates, N. and J. Pillinger. 2019. International Health Worker Migration and Recruitment: Global Governance, Politics and Policy: Routledge.

Zimmerman, H.L., M. Mülhausen and E. Tuck. 2019. Attacks on healthcare in conflict: generating attention in the modern information landscape. Medicine, Conflict and Survival 35, no 1: 12-42.

Zywert, K. and S. Quilley. 2018. Health systems in an era of biophysical limits: The wicked dilemmas of modernity. Social Theory \& Health 16, no 2: 188-207. 


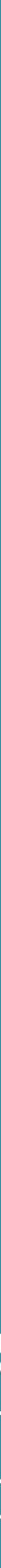




\section{THE SOCIAL AND ECONOMIC RELEVANCE OF THE RESEARCH}

The social and economic relevance of the research and studies embedded in the chapters of this thesis is considerable. The challenge of Health Workforce (HWF) development has been a core consideration for national governments and multilateral agencies alike. Despite the advance of global public health and medical interventions, several bottlenecks hinder the development and functioning of strong and equitable health systems. The absence of a strong HWF being one of them. The state and complexity of HWF development and mobility across sectors, institutions, professional cadres, and nations is a recurrent theme in the teaching of global health and public health students. They identify it as a priority concern for governments and non-governmental health actors alike. This thesis has indicated the gap between this social demand for, and public health needs of, having a strong HWF in place while at the same time, economic policy choices and financial investment are constrained in many settings.

The thesis incorporates publicly identified and publicly financed research assignments that look into the governance, policy, and political constellations of HWF actions as well as broader global health developments. The HWF study in Guinea in chapter 3 has been financed as part of a bilateral scientific development cooperation and capacity-building program between the Belgian and Guinean governments. The comparative tracing study on HWF policy actions was funded by, and conducted in close collaboration with the World Health Organization's (WHO's) HWF department and was aligned with the wHO's $12^{\text {th }}$ General Programme of Work, 2014-2019. The first of the two studies in chapter 4 was initiated and financed through development cooperation funding by the Dutch government as well as the European Union (EU) while I was still working at the civil society organization Wemos, and the second when I was specifically working with the EU on a project to advance HWF development in an equitable manner (HealthWorkers4All, 2013-2016). Both studies were published in academic journals on invitation by the wHO itself (on the Global Code of Practice) or by scholars who were analyzing the wHO governance reform processes and its debates (on democratizing the WHO) at length. The study in chapter 5 on the securitization of health and Attacks Against Health Care Workers in Conflict (AHCC) engages with an international debate and attention to AHCC that has increased after the eruption of the Syrian conflict and in relation to violence against health care workers during the Ebola outbreaks in 2014-2015 and 2018-2020. This study specifically aims to provide insight into why attacks against health care workers have increased rather than arguing that they should stop under humanitarian law. This study was not commissioned or funded by third parties. The study in chapter 6 was funded under, and was the final research output of, a Horizon 2020 research grant by the European Commission for the research program 'Goals and Governance for 
Health' (Go4Health, 2013-2016). This program followed the EU's strategic objective to provide scientific guidance to develop the Sustainable Development Goals (SDGs) and to help shape the EU's policy priorities engaging with the Sustainable Development Agenda. Finally, the relevance of the discussion chapter (chapter 7) and its analysis on the research questions, by including a political-economy framework (the globalization paradox and its trilemma) provides an elaborated perspective on the possibilities and limitations of Global Health Governance (GHG) and advancing HWF goals within the current Sustainable Development Agenda. It argues for transformative pathways beyond and outside current international collaboration regarding health systems and its workforce by explicitly embarking on an alternative circular economy and respecting the planetary boundaries approach. It eventually considers economic Degrowth options and thinking through what this implies for health systems development.

\section{TARGET GROUPS OUTSIDE ACADEMIC GIRGLES}

This study has engaged with, and in several ways targeted groups outside the academic circles. I have discussed several policy and governance components on numerous occasions and platforms with, amongst others, policymakers from multilateral organizations such as the WHO, World Bank, UN Children's Fund (UNICEF), Global Health Workforce Alliance (GHWA), Global Fund (GF), etc. I have engaged with program officers and Human Resources for Health (HRH)-policymakers from several countries as part of the tracing study and debates on workforce migration. Given the specific focus on Guinea, our research team has been engaging with policymakers, school directors, HWF managers, and professionals at national and local levels. The several articles in the chapters were also being discussed via ongoing engagements with civil society networks and non-governmental organizations (NGOs), both in Europe and in Low- and Middle-Income Countries (LMICs). This includes NGO development networks like Medicus Mundi International - Network health for All!, Médecins Sans Frontières (MSF), African Medical and Research Foundation (AMREF), OXFAM, and Intrahealth International as well as civil society activists groups like the People's Health Movement. There has been active collaboration with international labor unions such as Public Services International on the HWF migration challenge. The AHCG paper addresses humanitarian health care workers, asking them to reflect on their role in times of conflict. I have participated in several non-scientific panels discussing HWF development with representatives from professional associations such as the International Council of Nurses, the World Medical Association, and the International Federation of Medical Students' Associations. The WHO governance reform and global health priorities for the SDGs have been discussed with representatives from national diplomatic missions to the United Nations (UN), 
the European Commission as well as policymakers from ministries of development cooperation, foreign affairs, and finance from several, mainly European, countries. Health workforce governance requires complex, inter-sectoral approaches, involving a range of actors. I have engaged extensively with the actors mentioned above during the time of research and writing.

\section{ACTIVITIES AND PROGESSES}

The study and its several components have been analyzed and discussed as part of international policy debates on HWF governance, migration and global health reforms. The WHO's Code of Practice on the International Recruitment of Health Personnel was adopted by the World Health Assembly in 2010. I was already involved, as a civil society actor, in the original policy shaping and dialogue that led to the global Code. Since then there have been policy debates about its implementation, including formal reviews on the relevance and effectiveness of the code every 5 years. The paper in chapter 4 on implementation of the code in the European and African region was used as evidence for the wHO's first round of review in 2015. Our study on Global Skills Partnerships and health workforce migration, referred to in the discussion, has been used as evidence in the ongoing second review of the Code in 2020.

The policy tracing study on HRH commitments made by 57 government and other actors at the $3^{\text {rd }}$ global forum on HRH in Recife, Brazil has been part of the accountability mechanisms that were established under the GHWA during the first forum in 2008. The study traced HRH actions made during the period between the $3^{\text {rd }}$ and $4^{\text {th }}$ Global Forum. It was presented at the $4^{\text {th }}$ Global Symposium on Health Systems Research, Vancouver in 2016 as well as the $4^{\text {th }}$ Global Forum on HRH, Ireland in 2017. The latter entailed a panel discussion with government as well as NGO representatives about possibilities and challenges moving HRH actions and investments forward. During this $4^{\text {th }}$ Global Forum, specific attention was given to the countries in West-Africa, including Guinea, and the international cooperation required for building resilient health systems and investing in its HWF after the Ebola outbreak in 2014-2015.

The paper on the wHO governance contributes to a longer debate on reform and financing of the wHO, with the need for the wHO to become more autonomous, inclusive, and effective. The debate on the governance of the wHO in relation to other actors, including how to avoid conflicts of interests, has been taking place since 2010. It has been discussed during and around the World Health Assembly (WHA), both formally and in side-events, as well as in several global health fora. Financing and governing the WHO as the key normative actor in global health remains very relevant 
but, as this thesis indicates, is under considerable pressure given the fragility of multilateralism and forces of financialization, hyper-globalization, and countries choosing national interests and sovereignty over global public goods.

In 2015, I presented the GHG and SDG draft study at a panel during the annual European Health Forum, Austria as well as during the European Global Health Policy Platform. Both are places where policy, strategy, and science are discussed by multiple actors working on European Global Health issues. The final seminar of the Go4Health Horizon 2020 project, including discussion of its final outcomes, took place at the Prince Mahidol Award Conference, Thailand in 2016. This is likewise a leading annual global health forum for policymakers, practitioners, and scientists.

The securitization of health and AHCC paper was discussed in 2018 during a seminar hosted by the International Peace Institute in Geneva with the title: "Doctors in War Zones: International Policy and Healthcare During Armed Conflict”. In 2016 and 2017, I have participated in two policy dialogues hosted by the Graduate Institute Geneva and led by Prof. Kickbusch, on the topic of 'overcoming the gridlock in GHG.' In these dialogues, I have put forward some of the challenges of moving GHG forward in relation to health systems development. The paper on "moving the health workforce agenda beyond economic growth" was presented in 2018 at the $4^{\text {th }}$ People's Health Assembly, a main global health activist network in Bangladesh.

The debate on workforce governance and migration is further pursued under the International Platform on Health Workforce Mobility, co-governed by the wHO, the International Labour Organisation (ILO), and the Organisation for Economic Cooperation and Development (OECD). I participated in this platform representing the Medicus Mundi International network. In 2019, I was part of the team conducting a study on addressing health workforce mobility in the European region, funded by the Open Society Foundations. This initiative will be continued as a new civilsociety-led platform addressing the inequities in health workforce mobility.

\section{INNOVATION}

Innovation in this study exists in the fact that it is a cross-disciplinary approach and boundary spanning as such. I have applied a political-economic framework ('The globalization paradox') as well as integrated reflections on the cosmopolitan outlook, which is a socio-philosophical perspective on the challenges in modern societies and how to overcome that in an era of reflective modernity. By doing so, I have added new conceptual perspectives on HWF development that traditionally follow public health and labor economics theory including scientific methods that 
analyze health systems mainly within their national boundaries. The relevance and innovation of my study lie in the fact that it shows there are inherent contradictions and limitations in moving the global HWF agenda forward via economic cooperation ("inclusive economic growth") in times of globalization. HWF development requires to be delinked from Gross Domestic Product (GDP)-growth and fiscal space considerations. The understanding and recommendation to develop, implement, and analyze health systems that respect planetary boundaries and follow circular economic pathways, and are hence a post-capitalist construct, is an innovative and potentially transformative approach to collaborate on global HWF development.

\section{IMPLEMENTATION OF PLANS}

This element is likely the most challenging outcome. It will not be easy to implement plans as a way to 'improve' current workforce policies or development. There are short-term options, low-hanging fruits that have been identified in several studies. These have much to do with improving representation and legitimacy, as well as recognizing shared priorities in financing and governance of health systems, within and beyond national borders. However, the transformation towards Degrowth and circular economies, including in the workforce domain will require 'tipping points', certain moments of crisis and opportunity that facilitate such new ways of collaboration. Perhaps the current COVID-19 pandemic might become such a 'cosmopolitan' moment. These political windows will likely open as the climate crisis and social disruption will deepen over the coming years. 'Local' networks of sharing, caring, and solidarity will morph into a new understanding of how to organize our health systems. Nevertheless, these 'localized' experiences that one sees now already emerging in several cities and countries in the world also require sustained global cooperation on public goods, progressive climate policies, and ensuring international social protection, including for health care. There is a slow trend towards promoting and embarking on 'New Green Deals' both within the EU, United States (US) and arguably also in China. It remains a question of how 'inclusive' and 'transformative' these policies and pathways actually will be, what this will imply for health employment, and to what extent Low-income Countries (LICs) will benefit from it. I hope that this thesis has contributed to advance thinking and will transition to a new health workforce, fit for the $21^{\text {st }}$ century, standing up and being part of a global drive towards social equity and ecological stability. 


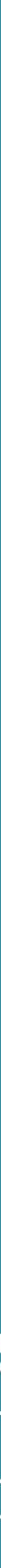


Over the last few decades, the global health workforce (HWF) gap has increased. This gap concerns the skilled HWF required for providing essential health care services across the world in an equitable manner. This thesis takes a cosmopolitan outlook, as coined by Ulrich Beck to describe a reflexive modernity, to study what is required to develop the global health workforce in an equitable manner. It looks into principles and policies of global health governance to assess what has been done to strengthen the health workforce. It also shows that there is a paradox in economic globalization, which leads to a structural problem to invest (sufficiently) in the health workforce at the national level. Via different methodologies, several levels of global health policy and health workforce development are studied. This includes a comparative policy analysis between countries as well as a specific study on health workforce investment in post -Ebola Guinea. Institutional reform of the WHO is studied alongside an analysis on the implementation of wHO's Code of Practice on the international Recruitment of Health Personnel. The implications of the securitization of health policy on attacks against humanitarian health workers are researched. The thesis includes a critical analysis of the current resilience focus in health systems development. It analyses to what extent global health approaches in the Sustainable Development Goals are grounded in the Right to Health.

The discussion then outlines the democratic space to reform and strengthen health workforce development across the different policy levels of global health governance. This is possible with a more cosmopolitan, transnational outlook to the health workforce challenge and international labour migration. This requires that countries take a shared sovereignty approach and find ways to regulate economic globalization so that it benefits the public good rather than the wealth of a few. However, current policy trends suggest that countries move away from these principles, instead of towards them. The thesis ends with suggestions on how to move beyond this 'gridlock' in global health workforce cooperation. It argues for moving beyond economic growth as a policy imperative, and instead take into account the planetary boundaries and social foundations as a basis for future global health workforce governance, known as the Doughnut Economics model. 


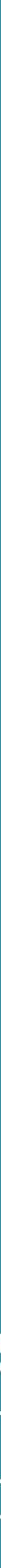


PERSONALIA:

Name: $\quad$ Remco Adrianus Maria van de Pas

Birthday: $\quad 11^{\text {th }}$ of July 1977, Berkel -Enschot, The Netherlands

\section{EDUCATION AND ACADEMIC DEGREES:}

Maastricht University \& Institute of Tropical Medicine Anwerp (2015 -2020)

KIT \& Vrije Universiteit (VU) Amsterdam, The Netherlands (2008-2010)

Royal tropical institute (KIT) Amsterdam, The Netherlands (2005)

University Medisch Centrum Nijmegen (UMCN), The Netherlands (2002-2004)

UMCN Nijmegen, The Netherlands (1997-2002)
Doctor of Philosophy (PhD) - candidate

Thesis: A cosmopolitan outlook on health workforce development

Master of International Public Health (MSc.IH)

Thesis: Human resources for health, opportunities and challenges in the Indonesian province of Papua

Netherlands Course on Hygiene and Tropical Medicine

Doctor of Medicine (MD)

Final rotation in social medicine via Nijmegen Institute for International Health (6 months). Placement in a PHC clinic run by NGO Médicos por el Derecho a la salud, Morazan, El Salvador Master Degree Medical Sciences

Thesis: The density of Dengue vector Aedes Aegypti and a Knowledge-Attitude-Practice study on community prevention. University Padjadaran, Dept. Parasitology. Bandung, Indonesia 


\title{
PROFESSIONAL EXPERIENCE:
}

\author{
May 2014 - to date
}

Institute of Tropical Medicine, Antwerp - Senior Research fellow (0.5 FtE)

Research, teaching and policy advise on health systems development and global health policy

Coordinator of the Emerging Voices for Global health (EV4GH) training course 2014. Scientific collaborator in the EC FP7 research program Go4 Health (2014-2016); Europe Aid program SPEED (Makerere University School of Public Health, Uganda 2015-2017); Belgian -Guinean institutional collaboration on health systems and workforce policy development (from 2017 onward.) Principle Investigator of a WHO comparative policy tracing study on health workforce policy actions in LMIC (2016-2017).

Lecturer in Master programme of Public Health and coordinator of two postgraduate courses (globalization and health, global health) at ITM (5 ECTS) and Sciences Po, Paris School of International Affairs (PSIA, 6 ECTS).

Associate editor of academic journal BMC Human Resources for Health.

\section{May 2019 - To date}

Maastricht University, FHML - Lecturer Global Health (0.5 FtE)

Lecturer and tutor in Global Health Master's program with a focus on the nexus between health governance, development, health systems, economics, politics and ecology. Supervision of Master Thesis research.

Associate editor of academic journal BMC Globalization and Health.

\section{May 2017 - April 2019}

Maastricht University - Academic Coordinator Maastricht Centre for Global Health (0.5 FtE)

Coordinator of a multi-disciplinary, research and educational centre for high quality research, training and capacity building in global health. Initiation and coordination of collaborations with university networks in Georgia, Iran (E+ project INPACT 2020-2023) Bangladesh (E+ project TRANS4M-PH 2019-2021), Indonesia, Canada and Europe.

\section{October 2014 - To date}

Clingendael, Netherlands Institute of International Relations - Senior Visiting Fellow

Expert advice, research and analysis on global issues and global health development from a foreign policy and international relations perspective. 


\section{September 2013- To date}

Medicus Mundi International - Network Health for All! (MMI) - Vice-president

Board member of a network of development- , advocacy- and research organisations working in the field of global health and international health cooperation. MMI is an NGO network in official relations with WHO. Steering Council member of the Geneva Global Health Hub. Steering Council member People's Health Movement (2012-2018).

\section{March 2014}

Kerk in Actie (NGO) - Consultant

Assessment of health program and strategic directions in Papua, Indonesia. Analysis of health and human rights situation West Papua as input for periodic review of the UN human rights council.

\section{July 2009 - April 2014}

Wemos Foundation - Health Policy Advisor

Research and analysis on global health policies. Thematic focus on multilateral and EU health policies, governance of the WHO, social determinants of health and policy coherence, health workers migration and retention. Advocacy and policy advice in cooperation with international networks and policymakers in WHO, Ministries of Health, Development Cooperation and public health professionals. Writing and implementing development proposa on health workforce policy for EU (EuropeAid) programs.

\section{February 2010- February 2014}

Dokters van de Wereld (Médecins du Monde - Netherlands) - President of the board

Governance and leading international NGO. Representing organization in internal and external networks. Strategic development of the NGO having programs in Netherlands, Bangladesh, Myanmar and Indonesia.

\section{January 2012 - February 2014}

Nationale Commissie Internationale Samenwerking en Duurzame Ontwikkeling (NCDO) Researcher

Research assignment analyzing trends in global health, its relevance, governance and impact for the Netherlands. Publication on Global Health part of NCDO's research portfolio on globalization and development.

\section{August 2010 - September 2010}

Misereor (NGO) - Consultant

Assessment and program evaluation of PHC program of the Diocese in the Asmat district, Papua, Indonesia. 
October 2009 - June 2010

VU medical center - Scientific Officer

Assessing public health implications and feasibility of research into new Leprosy vaccination $(\mathrm{rBCG})$

\section{May 2009 - July 2009}

World Health Organization - Consultant

Stocktaking and desk research on human resources for health strategies \& policies in 57 crisis countries. Coordinated by KIT Royal Tropical Institute.

\section{December 2005 - 2008}

Médecins du Monde-Medical and general coordinator

PHC, health systems strengthening and prevention of infectious diseases (TB, STI, HIV/AIDS) program in highlands district of Papua, Indonesia. Program coordination and evaluation with authorities, civil society actors and donors (EuropeAid and CordAid). 2007-2008

Assessment, initiation of community health and PHC program in former conflict area of Aceh province, Indonesia. 2006

Humanitarian health program in Kalma IDP-camp and Kass region, Darfur, Sudan. Supervision of Primary Health Clinic. Coordination with health authorities, NGOs and medical teams. 2006

\section{September 2004 - November 2005}

RIAGG-Rotterdam, Department of transcultural and social psychiatry-Medical Doctor

Medical consultations in (emergency) psychiatric care and risk reduction services for migrants and refugee populations. 


\section{PUBLICATIONS}

For a complete overview of publications, presentations and technical reports see profile at ResearchGate https://www.researchgate.net/profile/Remco_Van_De_Pas

\section{Publications in academic journals:}

- Van de Pas R, Rusthon S, Krumeich A, Townend D. The securitization of health and humanitarian space: Health workers under attack? Medicine, Conflict and Survival (2020, under review)

- Van Damme W, Dahake R, Delamou A, Ingelbeen B, Wouters E, Vanham G, van de Pas R, Dossou JP, Ir P, Abimbola S, Van der Borght S. The COVID-19 pandemic: diverse contexts; different epidemics-how and why?. BMJ Global Health. 2020 Jul 1;5(7):e003098.

- Harmer A, Leetz A, Eder B, van de Pas R, Gepp S. Time for WHO to declare climate breakdown a PHEIC?. Lancet 2020 Jul 4;396(10243):23-4.

- Townend D, Van de Pas R, Bongers L, Haque S, Krafft T, Van de Pas R, Pilot E, Schröder-Bäck P, Shaw D, Stahl N, Wouters B. What is the Role of the European Union in the COVID-19 Pandemic? June 2020. Medicine and Law. 39(2):249-268

- Harmer A, Eder B, Gepp S, Leetz A, van de Pas R. WHO should declare climate change a public health emergency. BMJ. 2020 Mar 30;368.

- Paul E, Brown GW, Ensor T, Ooms G, van de Pas R, Ridde V. We shouldn't count chickens before they hatch: results-based financing and the challenges of costeffectiveness analysis. Critical public health. 2020 Jan 4:1-6.

- Kolie D, Delamou A, van de Pas R, Dioubate N, Bouedouno P, Beavogui AH, Kaba A, Diallo AM, Van De Put W, Van Damme W. 'Never let a crisis go to waste': postEbola agenda-setting for health system strengthening in Guinea. BMJ Global Health. 2019 Dec 1;4(6).

- Van de Pas R, Kolie D, Delamou, A, Van Damme W. Health workforce development and retention in Guinea: a policy analysis post-Ebola. Human resources for health, 2019 17(1), 1-12.

- Flamig K, Decroo T, van den Borne B, van de Pas R. ART adherence clubs in the Western Cape of South Africa: what does the sustainability framework tell us? A scoping literature review. Journal of the International AIDS Society 2019 22(3), e25235.

- Van de Pas R, Mans L, Bemelmans M, Krumeich A. Framing the health workforce agenda beyond economic growth. Int J Health Policy Manag. 2018 May 16.

- Van Belle S, Van de Pas R, Marchal B. Queen bee in a beehive : WHO as metagovernor in global health governance. BMJ Global Health, Vol. 3, 2018, p. e000448.

- Van de Pas R, Veenstra A, Gulati D, Van Damme W, Cometto G. Tracing the policy implementation of commitments made by national governments and other entities at the Third Global Forum on Human Resources for Health. BMJ global 
health. 2017, 1;2(4):e000456

- Steurs L, van de Pas R, Delputte S, Orbie J. The Global Health Policies of the EU and its Member States: A Common Vision? International Journal of Health Policy and Management 2017, (6):1-10

- Van de Pas R, Ashour M, Kapilashrami A, Fustukian S. Interrogating resilience in health systems development. Health policy and planning, 2017 23;32(suppl_3):iii88-90

- Mans L, van de Pas, R. Marschang S. Civil society contributions to a sustainable health workforce in the European Union. WHO regional publications. European series, 2017 3(3):514-522

- Steurs L, van de Pas R, Decoster K, Delputte S, Orbie J. Role of the European Union in global health. The Lancet Global Health. 2017 Aug 1;5(8):e756.

- Ooms G, Flores W, Mulumba M, Sarker M, Van de Pas R, Jahn A. False and real, but avoidable, dichotomies. Lancet 2017;390(10095):647

- Van Belle S, Van de Pas R, Marchal B. Towards an agenda for implementation science in global health: there is nothing more practical than good (social science) theories. BMJ global health, 2017 2(2), p.e000181

- Legge DG, Schuftan C, Baum FE, van de Pas R, Sanders D, Hanson L. McCoy D, Sengupta A. Comment-WHO's weakness is not technical, but due to lack of accountability. BMJ Global Health, 2017 2(Suppl 1), i2-i3.

- Van de Pas R, Hill PS, Hammonds R., Ooms G, Forman L, Waris A, Brolan CE, McKee M, Sridhar, D. Global health governance in the sustainable development goals: Is it grounded in the right to health? Global Challenges, 2017 1: 47-60

- Van de Pas, R. Global Health in the Anthropocene: Moving Beyond Resilience and Capitalism. Comment on "Health Promotion in an Age of Normative Equity and Rampant Inequality. Int J Health Policy Manag, 2017 6(x),1-6

- Ooms G, Van de Pas R, Decoster K, Hammonds R. Thinking Out of the Box: A Green and Social Climate Fund. Comment on "Politics, Power, Poverty and Global Health: Systems and Frames" Int J Health Policy Manag, 2017 6(x), 1-4.

- Van de Pas R, Kolie D, Delamou A, Van Damme W, Van Belle S, Human resources for health. Lancet, 388 (10063) 2016. 2993-2994

- Van de Pas R, Mans L, de Ponte G, Dambisya Y. The Code of Practice and its enduring relevance in Europe and Eastern and Southern Africa. Human Resources for Health 2016, 14 (Suppl 1):30

- Van de Pas R, van Belle S. Ebola, the epidemic that should never have happened. Global Affairs, 2015. Vol 1(1): 1-6

- Battams S, van Schaik L, van de Pas R. The EU as a Global Health Actor: Policy Coherence, Health Diplomacy and WHO Reform .European Foreign Affairs Review, 2014 19. Issue 4, pp. 539-561.

- Van de Pas R, Tijtsma A. Rijke landen pak Ebola aan! Medisch Contact 27 Nov. 2014. $2380-81$ 
- Van de Pas R, van Schaik L. Democratizing the World Health Organization. Public Health, Volume 128 , Issue 2 , 2014.195-201

- SJ Rees, R. van de Pas, D. Silove and M. Kareth. Health and human security in West Papua. MJA 2008; 189: 641-643

- R. van de Pas. The effects of low human security on the health status of a struggling population. Do health indicators matter? J R Coll Physicians Edinb 2008; 38:3-4

- R. van de Pas. Darfur- dependent population at risk of another catastrophe. BMJ 2006;333:846-847

\section{Edited Book Chapters:}

- Van de Pas R, Ssennyonjo A, Criel B. How are developments at Regional and Global level influencing progress towards UHC in Uganda? Implications for Ensuring Good Health and Wellbeing. in Universal Health Coverage in Uganda, Looking Back and Forward to speed up the progress. Makerere University, SPEED project, European Union, 2018. 3; 51-72

- Baine SO, Ssennyonjo A, Remco van de Pas, Ssengooba F. Pooling Resources and Health Risks for UHC Advancement in Uganda: An Assessment of Current Mechanisms and Policy Options in Universal Health Coverage in Uganda,Looking Back and Forward to speed up the progress. Makerere University, SPEED project, European Union, 2018. 9; 184-204

- Van de Pas R, Dentico N. The Givil Society Perspective on the EU's Role in Global Health. in Emmerling, Thea, Ilona Kickbusch, and Michaela Told, eds. The European Union as a Global Health Actor. Vol. 4. World Scientific. 2016. 15; 313-328.

- Mans L, van de Pas R, Schwarz T, Sanders D. The global health workforce crisis. in Global Health Watch 4: An Alternative World Health Report. Zed Books p 158-166. 2014

- Van de Pas R. Mondiale Gezondheid. Vogelgriep, overgewicht, antibioticaresistentie en andere kwalen. Globaliseringsreeks no 11. NCDO . 2014

- Mans L, Van de Pas R, de Ponte G. Civil society's contribution and advocacy for implementing the WHO Global Code in the European Region: Six country case studies. in Siyam A, Del Poz M. (eds.) Migration of health workers: The WHO Code of Practice and the Global Economic Crisis. World Health Organization. p 17-29 May 2014. 


\section{Other publications, technical reports \& blogs:}

- $\quad$ Contributor International Health Policies blog. ITM Antwerp

- Van de Pas R. The Rich Man's Disease. Globalisierungsparadoxon und Coronavirus. IPPNW forum 22-23. June 2020

- Van de Pas R. Globalization Paradox and the Coronavirus Pandemic. Clingendael Research report. Clingendael. May 2020

- Van de Pas R. The Coronavirus pandemic and the irrelevance of the SDGs. Ghent Centre for Global Studies. April 2020

- Van Schaik, van de Pas, van Loo O, van den Berg P, Boer de M. Corona underpins the importance of global health arrangements. Clingendael. April 2020

- Van de Pas. Responding to the coronavirus outbreak. Clingendael March 2020

- Hinlopen C, van de Pas R. Implementing the Global Compact for Migration: unintended consequences of skills partnerships in the health sector. Policy Paper 2020. Friedrich Ebert Stiftung.

- Mans L, Milicevic MS, Guldemann H, Van de Pas R. Health Workforce Mobility and the European Principle of Solidarity in Health Systems. Policy paper. 2019 Open Society Foundations

- Van de Pas R, Mans L. Global Skills Partnerships \& Health Workforce Mobility: Pursuing a race to the bottom? Discussion paper for a side-event of the Intergovernmental Conference to Adopt the UN Global Compact for Safe, Orderly and Regular Migration. Public Services International. December 2018 Marrakesh. Morocco.

- Affun C, Ricarte B, Van Belle S, Van Damme W, Van De Pas R, Van De Put W. Primary health care and health emergencies. Background paper for the Alma Ata 40 Years Global Conference on Primary Health Care, Astana, Kazachstan, 25-26 October 2018. WHO, Unicef, Ministry of Health Care, Republic of Kazakhstan.

- Labonté R, Sanders D, Van de Pas R. Whither the WHO Code in the Scrap the Cap campaign? The BMJ opinion. 18 June 2018

- Van de Pas R. Contribution to Right to Food in West Papua. Human Rights Report. 2018 International Coalition for Papua. April 2018

- Van de Pas R. Right to Health. in Human Rights in West Papua, Fifth report of the international coalition for Papua (ICP), 2017 3.1.81-16

- Affun C, Ricarte B, Van Belle S, Van Damme W, Van De Pas R, Van De Put W. Context and Crisis. Evidence on Coordination and Health Systems Strengthening (HSS) in Countries under Stress: a literature review and some reflections on the findings. Research report for WHO/WB UHC 2030 Alliance. 2017 ITM, Antwerp

- Kolié D, Van de Pas R, Van Damme W, Van Belle S, Delamou A. Beavogui AB. Analyse situationnelle du personnel de santé et des établissements de formation pour le renforcement du système de santé afin de guider la coopération au développement entre la Belgique et la Guinée. Rapport de Recherche, Mai 2017. 
IMT, Anvers \& Centre National de Formation et de Recherche en Santé Rurale de Maferinyah, Ministère de la Santé , Guinée.

- Van de Pas R. Schwarz R, Leschorn M. Health Cooperation: Its Relevance, Legitimacy and effectiveness as a contribution to achieving Universal Access to Health. Discussion Paper. Medicus Mundi International Network. October 2016

- Soors W, De Man J, Dkhimi F, van de Pas R, Criel B. Towards universal coverage in the majority world: the cases of Bangladesh, Cambodia, Kenya and Tanzania. P4H Knowledge-Learning-Innovation Brief No 1.2016

- R. van de Pas. Le Personnel de Santé et le Droit à la Santé. In «La santé, une marchandise? Les dangers d'une commercialisation des soins de santé Chapter: 5, Publisher: CNCD-11.11.11., pp.P28- 33. April 2016

- Vandenbogaerde A, Hammonds RM, Van de Pas R, Ooms G. Literature study on possible international financing mechanisms for Social Protection. Commissioned by 11.11.11. June 2015

- R. van de Pas. The Right to Health in West-Papua 2015. P54-60 of the fourth Human Rights report of the International Coalition for Papua (ICP).

- W Soors, J de Man, P Ndiaye. F Dhkimi, R van de Pas, B Criel. Technical Report: Towards universal coverage in the majority world. Transversal findings \& lessons learnt, a summary. ITM Full-text · Technical Report · Aug 2015.

- L. van Schaik, R.van de Pas. Transnational Governance and Democratic Legitimacy. The case of Global Health. Clingendael and The Hague institute for global justice . 2014

- R. van de Pas, A. den Boer. Access to medicines and principles of social justice. Contact no. 196 World Council of Churches. 4-7. Dec. 2013

- R. van de Pas. The right to health in Papua. Chapter 3.1 in: Human Rights in West Papua 2013. International Coalition for Papua.; p40-43. 2013

- R.van de Pas. Globalisation, EU policies and its impact on the Dutch health system. Briefing paper. Wemos. 2013

- $\quad$ Co-author. Special Mondiale Gezondheid. One World Magazine No.10 Dec. 2012; p69-82

- I. Wolffers, J. Pronk, R van de Pas. Door strafbaarstelling illegaal verblijf komen gezondheid van ongedocumenteerde personen en de volkgezondheid in het gedrang. Dokters van de Wereld. Feb. 2012

- Co-author. Delhi Statement. "Time to untie the knots - The WHO reform and the need for democratizing global health". Medicus Mundi International and others. May 2011

- Co-author. Wemos and C. Jansen. Chances for Change. Dutch measures to improve the global distribution of health personnel. Dutch alliance on human resources for health. Dec. 2010

- $\quad$ E. Verheul, R. van de Pas. Health is a political issue. Global Medicine 10. IFMSA-NL. May 2010. p27-29 
- $\quad$ E. Berkhout, R.van de Pas, I. Wolffers. Gezondheidszorg is geen produkt. Vice Versa.Vakblad ontwikkelinsgssamenwerking. jaargang 43, 2009-6. p6

- R. van de Pas. People's Health in Darfur-Dependent communities. People's Health Movement. 2006 


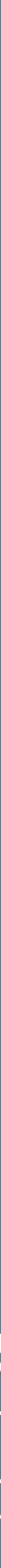


I am grateful to many people for their ongoing support, advice and friendship while conceptualizing, writing and finalizing this thesis. This happened to an extent via the professional organizations, academic institutions and activist networks I have been involved in. I like to mention these organizations and persons including for what they have meant to me. Apologies in advance for overlooking people in this overview. There have been simply too many that influenced my thinking and development. I have been privileged to be able to study, work, travel and live across different countries, contexts and places over the last 20 years. These unique connections, collegiality and friendships constitute the real wealth of my cosmopolitan life. It also implies that I, perhaps unconsciously, have lost the connection with some places, organizations and friends. I am hence not a stranger to the globalization paradox. This may be a good place to reflect on it.

The humanitarian NGO Médecins du Monde, specifically its Dutch branch Dokters van de Wereld, enabled me to work for several years in remote, conflict-affected, settings on primary health care, infectious diseases prevention and local health systems strengthening. These intense periods, being stationed in a refugee camp in Darfur, Sudan and in the central highland districts of Aceh and West-Papua, Indonesia have been very formative for understanding the possibilities, limitations and injustices of under -resourced and neglected health care settings. In a later period, when I was involved in the governance and management of this NGO network, I learned a lot about the leadership required and politics involved in making international cooperation happen. Seriana van den Berg, Arianne de Jong, Petra Wisse, Patricia Gaillard, Francoise Sivignon, Ineke Baas, Martin Kafiar, Flora Sainyakit, I Made Linggawijaya and Olaf Valverde have been wonderful colleagues during those years.

The international People's Health Movement (PHM), a social activist, academic and grassroots network, has been very important to me for learning and developing action on the structural determinants of health and the political-economy of international health policies. It has made me understand the root causes of health inequities and injustices, valuing the power of social mobilization and bottom-up action. Through involvement in its international People's Health University and the PHM steering council I got to know inspiring, wise and tenacious folks. These include the late David Sanders and Amit Sengupta, Chiara Bodini, Dave McCoy, Fran Baum, Jose Utrera, Wim de Ceukelaire, Julie Castro, Kumanan Rasanathan, Ronald Labonté, Anuj Kapilashrami, Bridget Lloyd, Andrew Harmer, David Legge, Lanny Smith, Eduardo Espinoza, Itai Rusike and many others. I especially want to mention Francoise Barten who was my mentor at Radboud University Nijmegen and provided the initial introduction to the PHM. She also encouraged me about a decade ago to pursue further $\mathrm{PhD}$ research. 
The Medicus Mundi International- Network Health for All! (MMI), a NGO and academic network has been an important enabler for involvement in several international health policy debates and processes with the wHO and beyond. My engagement as MMI board member has extended to The Geneva Global Health Hub (G2H2), a progressive platform to exchange on global health issues in Geneva. I like to thank Carlos Mediano, Christina de Vries, Martin Leschhorn and other (former) board members for their dedication and great exchanges. Thomas Schwarz has been the true unifying and organizing force of the MMI network and G2H2. He has been a partner in crime, in the best sense, over the last 10 years. Andreas Wulf, of Medico international, has been a dear friend and fellow activist. Nicoletta Dentico has provided passion and intellectual stimulation to our countless discussions.

The Wemosfoundation, in Amsterdam, has been a safe home for almost 5 years before moving into academia. I cannot express how important these years have been for developing a critical, autonomous, vision on global health issues, social justice, and its broader politics. Working at this NGO allowed me to bridge the evidence and knowledge field with policy activism. There, I developed the competence and right attitude, when dealing with global health governance and international workforce complexities. I am grateful that the organisation provided me with the trust and space to engage in these matters. Anke Tijtsma, Mariska Meurs, Annelies den Boer, Corinne Hinlopen and others have ensured a solid team that guided the organisation through a difficult period. A special thanks goes to Linda Mans, we have been friends since our collaboration on health workforce matters.

Gorik Ooms encouraged me to apply for a research position in the health policy unit of the Institute of Tropical Medicine, Antwerp. This unit has been the place where I have been able to mature academically. The exchanges with Gorik and Rachel Hammonds on the intersection between human rights law, international relations and global health governance laid the basis for this thesis. Wim van Damme has provided me with the trust, advice and space to develop an autonomous research focus. I much appreciate our discussions trying to 'make sense' of things. Kristof Decoster and Werner Soors have become close friends while rowing against the capitalist tide. Willem van de Put, Sara Van Belle, Bruno Marchal, Bart Criel, Isa Bogaert, Marjan Pirard, Josefien van Olmen, Annelies de Potter, Tim Roosen and many other colleagues have ensured a warm and stimulating working environment.

I have had the privilege to collaborate internationally with a diverse range of researchers and practitioners. These include Aloysius Ssennyonjo, Delphin Kolie, Alex Delamou, Prashanth NS, Albrecht Jahn, Peter Hill, Yibeltal Assefa, Stephanie Topp, Seye Abimbola, Meri Koivusalo, Fatemeh Ahmadi, Yoswa Dambisya, Michaela Told, Katerini Storeng, Rene Loewenson, Walter Flores and others. It has been fun working together with you and it provided many insights. 
The Global Health Studies section of the International Studies Association has been an enervating place for interdisciplinary exchange and debate. Thank you for the open discussions, Simon Rushton, Clare Wenham, Stefan Elbe, Owain Williams, Jeremy Shiffman, Julie Balen, Lies Steurs, Auriane Guilbaud and Adam Kamradt-Scott.

Louise van Schaik from Clingendael, Netherlands Institute for International Relations, facilitated an associate position, collaboration and debate on foreign policy and global health issues with Dutch and European actors. Especially during the Covid-19 pandemic this has become an intense cooperation. I really appreciate our joint work over the last decade.

During the last three years, the global health colleagues and affiliated researchers at Maastricht University have provided a place and community for interdisciplinary work as well as the supervision team for this thesis. Coming from a different professional and research discipline, I have greatly appreciated the exchanges with other perspectives. Thank you, Anja Krumeich and David Townend, for the patience, discussions and guidance. Nora Engel, Agnes Meershoek, Gonnie Klabbers, Thomas Krafft, Peter Schröder - Bäck, Bart Penders, Ricky Janssen, Alena Kamenshchikova, Preeti Sushama, Hellen Heutz and others have been great colleagues. Against all odds, we have been able to start a global health education project with our wonderful Iranian colleagues Amirhossein Takian and Reza Majdzadeh.

I must also thank WHO's health workforce department with whom I have cooperated frequently since the adoption of the Global Code of Practice on the international recruitment of health personnel in 2010. This includes James Campbell, Ibadat Dhillon, Giorgio Cometto, Rania Kawar, Pascal Zurn, Laurence Kodija. The global workforce complexity deserves a bigger team and more sustainable financing. Other wHO staff that deserve mentioning are Fahdi Dhkimi, Taina Nakari, Agnès Soucat, Marjolain Nicod and Leen Meulenbergs. I have over the years exchanged on WHO governance and global health policy with several UN mission delegates and experts including Eero lathinen, Canice Nolan, Bjorn Kummel, Gert-Jan Rietveld, Kevin McCarthy and Ilona Kickbusch. This has been insightful to help understand its complex dynamics and politics.

I have been privileged in facilitating research and educational work by talented students and young professionals. This continuing interaction and mutual learning with a next generation has been a crucial element of academic life. This includes Daniel Gulati, Milou Lustermans, Boutaina Emrani, Nathalie Rhodes, Jeffrey Quarsie, Myria Koutsoumpa, Maike Voss, Marco Angelo and several others. 
Barend Gerritsen and Marjolein Dieleman from KIT have provided crucial guidance and advice during and after my international public health studies. Ivan Wolffers has been an inspiration. Godelieve van Heteren, Koos van der Velden, Kerstin Klipstein, Leon Bijlmakers and Hans Hoogerzeil have been allies pushing global health policy and systems thinking forward in the Netherlands.

My parents, Frans and Ria van de Pas, my brother Michiel, his own family and the extended family have been actively supporting me throughout, in the background. They have always provided the trust and space that provided me the liberty to follow my path. The care of my parents for their granddaughter was much welcome while the parents were working or travelling. I hope I can be there more for you too.

There are some great friends I can rely on; Roel, Martijn, Sarah, Manon, Roland, Gabie and Peter. It is so wonderful that you have been there all those years, even though we see each other only infrequently. A special thanks goes to Richard van Galen and Jeanine Pothuizen. You have always provided a home when I needed it.

Thank you Katja for being there.

Yulia Sugandi, the mother of our daugther; your intellect, spirituality and dedication has been of great influence on me.

The uncountable exchanges and collaborations with a varied group of health workers in different settings have been the main driver to conduct this study and write it all down. I like to provide recognition and respect for these essential workers in society.

This thesis is dedicated to my daughter Ika van de Pas, faraway but close, always with me. 


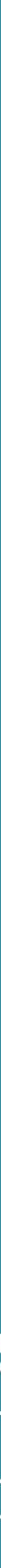




\begin{tabular}{|c|c|}
\hline 3GF & Third Global Forum \\
\hline AC & Assessed Contributions \\
\hline AHCC & Attacks Against Health Care Workers in Conflict \\
\hline AHPSR & Alliance for Health Policy and Systems Research \\
\hline AIDS & Acquired Immunodeficiency Syndrome \\
\hline AMR & Antimicrobial Resistance \\
\hline AMREF & African Medical and Research Foundation \\
\hline ANSS & National Health and Safety Authority \\
\hline ASEAN & Association of Southeast Asian Nations \\
\hline ATS & Nurse-Assistants \\
\hline $\mathrm{AU}$ & African Union \\
\hline BMGF & Bill and Melinda Gates Foundation \\
\hline BRICS & Brazil, Russia, India, China, South Africa \\
\hline CHW & Community Health Workers \\
\hline CHWF & Community Health Workforce \\
\hline $\mathrm{CIA}$ & Central Intelligence Agency \\
\hline CNFRSR & Centre National de Formation et de Recherche en Santé Rurale, Maferinyah \\
\hline CSDH & Commission on Social Determinants of Health \\
\hline CSI & Civil Society Initiative \\
\hline $\mathrm{CSO} / \mathrm{s}$ & Civil Society Organization/s \\
\hline DAH & Development Assistance for Health \\
\hline DFID & Department for International Development \\
\hline DNA & Deoxyribonucleic Acid \\
\hline DRC & Democratic Republic of the Congo \\
\hline EB & Executive Board \\
\hline ECOWAS & Economic Community of West African States \\
\hline EEA & European Economic Area \\
\hline EMT & Emergency Medical Teams \\
\hline EQUINET & European Network of Equality Bodies \\
\hline ESA & Eastern and Southern African \\
\hline EU & European Union \\
\hline EVD & Ebola Virus Disease \\
\hline FCTC & Framework Convention of Tobacco Control \\
\hline FENSA & Framework of Engagement with Non-State Actors \\
\hline FES-CCOO & European Federation of Public Service Unions, Verdi \\
\hline FfD3 & Third International Conference on Financing for Development \\
\hline
\end{tabular}


FNOMCeO Italian National Professional Organization of medical doctors

FSP-UGT Spanish Trade Unions for Health Workers

GATS Global Agreement on Trade in Services

GDP Gross Domestic Product

GF Global Fund

GHD Global Health Diplomacy

GHG Global Health Governance

GHS Global Health Security

GHW Global Health Workforce

GHWA Global Health Workforce Alliance

GHWN Global Health Workforce Network

GKN Globalization Knowledge Network

GOARN Global Outbreak and Alert Response Network

GPGH Global Public Good for Health

GSP Global Skills Partnerships

HBP Health as a Bridge for Peace

HIC/s High-Income Country/Countries

HIV Human Immunodeficiency Virus

HPSR Health Policy and Systems Research

HRC Human Rights Council

HRH Human Resources for Health

HSR Health Systems Research

HSS Health Systems Strengthening

HW/s Health Worker/s

HWF Health Workforce

IAEG-SDGs Inter-agency and Expert Group on Sustainable Development Goal Indicators

ICESCR International Covenant on Economic, Social and Cultural Rights

ICRC International Committee of the Red Cross

IFI/s International Financial Institution/s

IHR International Health Regulations

ILO International Labour Organisation

IMF International Monetary Fund

IPASVI The Professional Federation of Nurses, Italy

IPHWM International Platform on Health Workforce Mobility

IPHWM International Platform on Health Worker Mobility

ITM Institute of Tropical Medicine, Antwerp 


\begin{tabular}{|c|c|}
\hline $\mathrm{LIC} / \mathrm{s}$ & Low-income Country/Countries \\
\hline LMIC/s & Low- and Middle-Income Country/Countries \\
\hline$M \& E$ & monitoring and evaluation \\
\hline MDG/s & Millennium Development Goal/s \\
\hline $\mathrm{MIC} / \mathrm{s}$ & Middle-income Country/Countries \\
\hline MoH & Ministry of Health \\
\hline MPs & Members of Parliament \\
\hline MPW & Medical Peace Work \\
\hline MSF & Médecins Sans Frontières \\
\hline NATO & North Atlantic Treaty Organization \\
\hline $\mathrm{NCD} / \mathrm{s}$ & Non-Communicable Disease/s \\
\hline $\mathrm{NGO} / \mathrm{s}$ & Non-governmental organization/s \\
\hline NHS & National Health Service \\
\hline NL & the Netherlands \\
\hline ODA & Official Development Assistance \\
\hline OECD & Organisation for Economic Co-operation and Development \\
\hline OOP & Out-of-Pocket \\
\hline OOPE & Out-of-Pocket Expenditure \\
\hline PAHO & Pan American Health Organization \\
\hline PEPFAR & The United States President's Emergency Plan for AIDS Relief \\
\hline PHC & Primary Health Care \\
\hline PHEIC & public health emergency of international concern \\
\hline SAP & Structural Adjustment Programs \\
\hline SARS & severe acute respiratory syndrome \\
\hline SDG & Sustainable Development Goal \\
\hline SDG 3 GAP & Global Action Plan for Healthy Lives and Well-being for All \\
\hline SDH & Structural Determinant of Health \\
\hline SDI & Sustainable Development Index \\
\hline SEARO & South-East Asia Region \\
\hline TiSa & Trade in Services Agreement \\
\hline TPP & Trans-Pacific Partnership \\
\hline TRIPS & Trade-Related Aspects of Intellectual Property Rights \\
\hline UHC & Universal Health Coverage \\
\hline UiO & University of Oslo \\
\hline UK & United Kingdom \\
\hline UN & United Nations \\
\hline
\end{tabular}




\begin{tabular}{ll} 
UNAIDS & United Nations Programme on HIV/AIDS \\
UNASUR & Union of South American Nations \\
UNFPA & UN Population Fund \\
UNFPA & United Nations Population Fund \\
UNGA & United Nations General Assembly \\
UNHCR & United Nations High Commissioner for Refugees \\
UNHEEG & United Nations High Level Commission on Health Employment and Economic \\
UNICEF & Growth \\
UNMEER & United Nations Mission for Ebola Emergency Response \\
UNSC & United Nations Security Council \\
US & United States \\
USD & United States Dollar \\
VC & Voluntary Contributions \\
WB & World Bank \\
WHA & World Health Assembly \\
WHF & World Health Forum \\
WHO & World Health Organization \\
WTO & World Trade Organisation \\
XR & Extinction Rebellion \\
\hline UNO & Und
\end{tabular}




